\title{
Precision Measurement of the Spin-dependent Asymmetry in the Threshold Region of Quasielastic ${ }^{3} \overrightarrow{\mathrm{He}}\left(\vec{e}, e^{\prime}\right)$
}

\author{
by \\ Feng Xiong \\ Submitted to the Department of Physics \\ in partial fulfillment of the requirements for the degree of \\ Doctor of Philosophy \\ at the

\section{MASSACHUSETTS INSTITUTE OF TECHNOLOGY}

September 2002

(C) Feng Xiong, MMII. All rights reserved.

The author hereby grants to MIT permission to reproduce and distribute publicly paper and electronic copies of this thesis document in whole or in part.

Author

Department of Physics

June 13, 2002

Certified by

Haiyan Gao

Associate Professor

Thesis Supervisor

Accepted by . .

Thomas J. Greytek

Associate Department Head for Education 


\title{
Precision Measurement of the Spin-dependent
}

\author{
Asymmetry in the Threshold Region of \\ Quasielastic ${ }^{3} \mathrm{He}\left(\vec{e}, e^{\prime}\right)$ \\ by
}

Feng Xiong

Submitted to the Department of Physics

on June 13,2002 , in partial fulfillment of the

requirements for the degree of

Doctor of Philosophy

\begin{abstract}
The first precision measurement of the spin-dependent asymmetry in the threshold region of ${ }^{3} \mathrm{He}\left(\vec{e}, e^{\prime}\right)$ was carried out in Hall A at the Jefferson Laboratory, using a longitudinally polarized continuous wave electron beam incident on a high-pressure polarized ${ }^{3} \mathrm{He}$ gas target. The polarized electron beam was generated by illuminating a strained GaAs cathode with high intensity circularly polarized laser light, and an average beam polarization of about $70 \%$ was achieved. The ${ }^{3} \mathrm{He}$ target was polarized based on the principle of spin-exchange optical pumping and the average target polarization was about $30 \%$. The scattered electrons were detected in the two Hall A high resolution spectrometers, HRSe and HRSh. The data from HRSh were used for this analysis and covered both the elastic peak and the threshold region. Two kinematic points were measured in the threshold region, one with a central $Q^{2}$-value of $0.1(\mathrm{GeV} / \mathrm{c})^{2}$ at an incident beam energy $E_{0}=0.778 \mathrm{GeV}$ and the other with a central $Q^{2}$-value of $0.2(\mathrm{GeV} / \mathrm{c})^{2}$ at $E_{0}=1.727 \mathrm{GeV}$. The average beam current was $10 \mu \mathrm{A}$, which was mainly due to the limitation of the polarized ${ }^{3} \mathrm{He}$ target.

The measured asymmetry was compared with both plane wave impulse approximation (PWIA) calculations and non-relativistic full Faddeev calculations which include both final-state interactions (FSIs) and meson-exchange currents (MECs) effects. The poor description of the data by PWIA calculations at both $Q^{2}$-values suggests the existence of strong FSI and MEC effects in the threshold region of ${ }^{3} \mathrm{He}\left(\vec{e}, e^{\prime}\right)$. Indeed, the agreement between the data and full calculations is very good at $Q^{2}=0.1$ $(\mathrm{GeV} / \mathrm{c})^{2}$. On the other hand, a small discrepancy at $Q^{2}=0.2(\mathrm{GeV} / \mathrm{c})^{2}$ is observed, which might be due to some $Q^{2}$-dependent effects such as relativity and three-nucleon forces (3NFs), which are not included in the framework of non-relativistic Faddeev calculations. Some preliminary results show that $3 \mathrm{NF}$ effects are indeed non-negligible
\end{abstract}


in the threshold region and more theoretical work is currently underway towards a better understanding of both effects. With the recent development and success of the chiral perturbation theory, it is hopeful that a future application of the resulting chiral $N N$ forces to electrodisintegration of ${ }^{3} \mathrm{He}$ might solve the present discrepancy.

Thesis Supervisor: Haiyan Gao

Title: Associate Professor 


\section{Acknowledgments}

This thesis could never have been completed without the help of many people and I would like to take this opportunity to express my deep gratitude towards all of them. On the other hand, it is simply impossible to list all of their contributions here and I apologize for any possible but unintentional omissions.

First of all, I would like to thank my thesis advisor, Professor Haiyan Gao, for her consistent encouragement and guidance through all these years. I am deeply impressed by her enthusiasm and devotion towards the field and has been constantly inspired by her physics insights and creative ideas. It has been a great pleasure for me to discuss with her over many interesting physics problems.

I would like to thank Dipangkar Dutta, a postdoctoral fellow in our group, and Wang $\mathrm{Xu}$, a fellow graduate student of mine, for their help with a lot of analysis issues. Dipangkar is remarkable in that he is always energetic and truly capable of multitasking. Wang impressed me a lot with his patience and his quiet way of thinking. I would also like to thank other group members, Ben Clasie, Chris Crawford, Bin Ma, Jason Seely, Hong Xiang and Lingyan Zhu for many interesting discussions. Chris is always eager to share with me his findings about some new software tools and I really learned a lot from him in this way. I am also deeply indebted to Professor Bill Donnelly and Professor Claude Williamson at MIT for sharing their valuable time and knowledge with me.

I would like to thank all JLab staff for making this experiment possible. Specificly, I would like to thank Drs. Jian-Ping Chen, Ole Hansen, Xiaodong Jiang, Mark Jones, Nilanga Liyanaga and John Lerose for their direct help with me. It was also a great pleasure to work with many fellow students at JLab, among them Alexandre Deur, Pibero Djawatho, Steffen Jensen, Ioannis Kominis and Karl Slifer. And certainly I would never forget those wonderful moments with Sebastien Incerti and Enkeleida Lakuriqi.

I would like to thank all my friends who are working or once worked on the 
penthouse of the Laboratory for Nuclear Science (LNS) at MIT for making it such a lovely workplace. Among them are Zhengwei Chai, Jiang Chen, Juncai Gao, Peter Monaghan, Bradley Plaster, Shiao-bin Soong, Yuan Xiao, Bin Zhang, Chi Zhang, Xiaochao Zheng, Yi Zheng and Jindong Zhou.

Many people at LNS helped me a lot during the last four years. I thank Stu Galley and Wolfgang Wander from the computer center for helping me troubleshoot many computer problems. I also thank Joanne Gregory for making all the travel arrangements between MIT and JLab for me.

Finally I would like to take the opportunity to thank my entire family for their love and support. My parents have never hesitated in supporting me to pursue a higher education, for which they have made countless sacrifices. And I am truly blessed with a cheerful wife who makes my life such an enjoyable journey. 


\section{Contents}

1 Introduction $\quad 19$

1.1 Physics Motivation . . . . . . . . . . . . . . . . . 19

1.2 Nuclear Interaction $\ldots \ldots \ldots \ldots \ldots$

1.2.1 Nucleon-Nucleon Potentials . . . . . . . . . . . . . . 23

1.2.2 Three-Nucleon Forces and Relativistic Effects . . . . . . . 25

1.2 .3 Meson-Exchange Currents . . . . . . . . . . . . . 28

1.3 Spin-Dependent Asymmetry in Quasielastic ${ }^{3} \mathrm{He}\left(\vec{e}, e^{\prime}\right) \ldots \ldots . . .31$

1.3.1 General Formalism for Inclusive Electron Scattering . . . . . . 32

1.3.2 PWIA Calculations . . . . . . . . . . . . . . . 37

1.3.3 Non-Relativistic Full Faddeev Calculations . . . . . . . . . . 41

2 The Experimental Apparatus $\quad 47$

2.1 Overview. . . . . . . . . . . . . . . . . 47

2.2 Polarized Electron Beam . . . . . . . . . . . . . . 50

2.2 .1 Beam source . . . . . . . . . . . . . . . . . 50

$2.2 .2 \quad$ Møller Polarimeter . . . . . . . . . . . . . . . 52

2.2 .3 Beam Current Monitors . . . . . . . . . . . . . 53

2.2.4 Beam Position Monitors . . . . . . . . . . . . . . 56

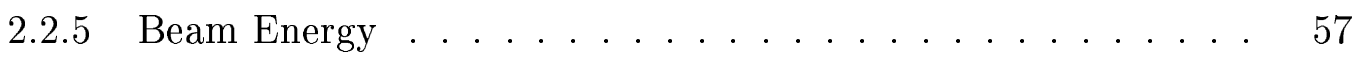

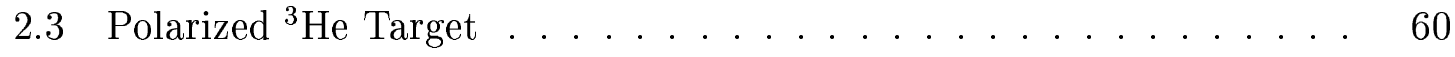

2.3.1 Optical Pumping and Spin Exchange . . . . . . . . 60 
2.3.2 The JLab Hall A Polarized ${ }^{3} \mathrm{He}$ Target . . . . . . . . . . . . 64

2.3.3 Target Polarization Measurement . . . . . . . . . . . 68

2.4 High Resolution Spectrometer . . . . . . . . . . . . . . . 72

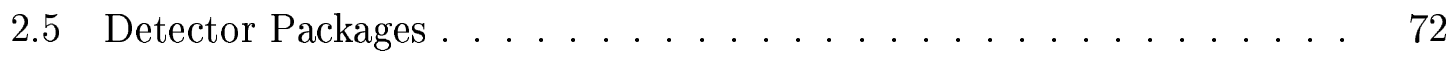

2.5.1 Vertical Drift Chamber . . . . . . . . . . . . . 73

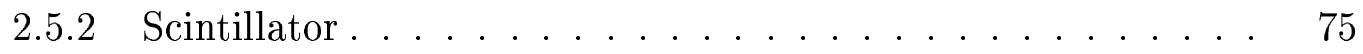

2.5.3 Gas Čerenkov . . . . . . . . . . . . . . . . 76

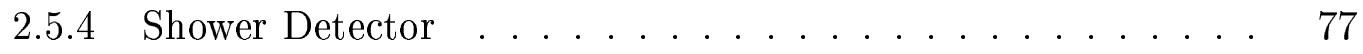

2.6 Data Acquisition System . . . . . . . . . . . . . . . . 79

2.6 .1 Trigger Electronics . . . . . . . . . . . . . . . 79

2.6 .2 Data Acquisition . . . . . . . . . . . . . . 82

$\begin{array}{lll}3 & \text { Data Analysis } & 87\end{array}$

3.1 Overview of Asymmetry Analysis . . . . . . . . . . 87

3.2 Experimental Raw Asymmetry _ . . . . . . . . . . . . . 89

3.3 Background Subtraction $\ldots \ldots \ldots \ldots$. . . . . . . . . 91

3.3.1 Empty Target and $\mathrm{N}_{2}$ Dilution Factors . . . . . . . . . 91

3.3.2 Empty Target and $\mathrm{N}_{2}$ False Asymmetry $\ldots \ldots \ldots$. . . 97

3.4 Monte Carlo Simulation . . . . . . . . . . . . . . . . . . 99

3.4 .1 Overview . . . . . . . . . . . . . 100

3.4.2 External Radiative Correction . . . . . . . . . . . . . . 101

3.4.3 Internal Radiative Correction . . . . . . . . . . 106

3.4.3.1 Schwinger Correction . . . . . . . . . . . . 110

3.4.3.2 Covariant Methods for Polarized Radiative Correction 110

3.4.4 Spectrometer Model and Acceptance . . . . . . . . . . 118

3.4.5 ${ }^{4}$ He Radiative Tail Simulation . . . . . . . . . . . . . . 121

3.5 Elastic Polarimetry . . . . . . . . . . . . . . . . . . . . . . . 121

3.6 Elastic Radiative Tail Dilution Factor and Asymmetry . . . . . . 124

3.7 Quasielastic Asymmetry Radiative Correction . . . . . . . . . 125 
3.8 Bin-Averaging and Acceptance Effects $\ldots \ldots \ldots \ldots \ldots$

3.9 Experimental Cross Section _ . . . . . . . . . . . . 126

3.9.1 ${ }^{3}$ He Density Determination . . . . . . . . . . . . . . 128

3.9.2 Deadtime and Detector Inefficiencies _ . . . . . . . 128

3.9.3 Spectrometer Acceptance . . . . . . . . . . . . . . . . . 129

3.9.4 Results for Experimental Cross Section . . . . . . . . . 129

4 Systematic Uncertainties 133

4.1 Systematic Uncertainties from False Asymmetries . . . . . . . . . 134

4.1.1 Helicity-Correlated Beam Current Shift . . . . . . . . . . . . 134

4.1.2 Helicity-Correlated Beam Position Shift . . . . . . . . . . 137

4.1.3 Data Acquisition Deadtime . . . . . . . . . . . 137

4.1.4 Trigger Efficiency . . . . . . . . . . . . . . . . 140

4.1.5 VDC Efficiency . . . . . . . . . . . . . . . . . . . . 142

4.2 Systematic Uncertainties from Background Subtraction . . . . . . 143

4.2.1 Empty Target and $\mathrm{N}_{2}$ Dilution Factors . . . . . . . . 143

4.2 .2 Pion Contamination . . . . . . . . . . . . . . . 143

4.3 Systematic Uncertainties from Simulation . . . . . . . . . . . . 146

4.3 .1 Elastic Form Factors . . . . . . . . . . . . . . 146

4.3.2 Quasielastic Response Functions . . . . . . . . . . . . . 146

4.3.3 Thickness of External Radiative Material . . . . . . . . . . 147

4.3.4 Spectrometer Acceptance . . . . . . . . . . . . . . . 147

5 Results and Discussion $\quad 149$

5.1 Physics Asymmetry and Comparison with Theoretical Calculations . 149

5.2 Theoretical Investigations _ . . . . . . . . . . . . . 157

5.2.1 Three-Nucleon Force Effects . . . . . . . . . . . . . 157

5.2 .2 Relativistic Effects . . . . . . . . . . . . . . . 164

5.2.3 Recent Development of Chiral Perturbation Theory . . . . . . 164 
5.3 Summary and Conclusion ................... 165

$\begin{array}{ll}\text { A Polarized Radiative Correction } & 167\end{array}$

A.1 Class Structure and General Description . . . . . . . . . 167

A.2 Structure of a Main Simulation Program . . . . . . . . . . . 171 


\section{List of Figures}

1-1 One-boson exchange Feynman diagrams that define the CD-Bonn NN

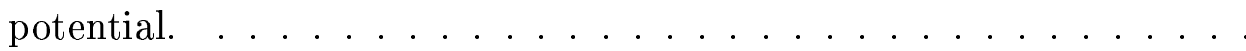

1-2 Fujita-Miyazawa three-nucleon force through the exchange of two pions between three nucleons, during which one of the nucleons is excited to

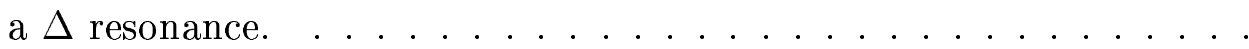

1-3 Feynman diagram representation of the isovector currents associated with $\pi$ and $\rho$ exchanges. Solid lines represent nucleons, dashed lines represent $\pi$ or $\rho$ mesons and wavy lines represent photons. . . . . .

1-4 Feynman diagram representation of the isoscaler $\rho \pi \gamma$ and isovector $\omega \pi \gamma$ transition currents. Solid lines represent nucleons, dashed lines represent pions while thick-dashed lines vector mesons $\rho$ or $\omega$, and wavy lines represent photons. . . . . . . . . . . . .

1-5 Feynman diagram representation of the $N N \rightarrow N \Delta$ and $N N \rightarrow \Delta \Delta$ transition interactions. Solid lines represent nucleons, thick-solid lines represent $\Delta$ isobars, and wavy lines represent photons. . . . . . .

1-6 A general $\left(e, e^{\prime}\right)$ spectrum. . . . . . . . . . . . .

1-7 The Feynman diagram for the first-order Born approximation. . . . . 34

1-8 The scattering plane. . . . . . . . . . . . . 36

1-9 The Feynman diagram in Plane Wave Impulse Approximation. . . . . 37 
2-1 An aerial view of Jefferson Lab accelerator site and the three experimental halls. . . . . . . . . . . . . . . 48

2-2 Schematics of JLab accelerator configuration. . . . . . . . . . 49

2-3 Hall A Configuration for E95-001. . . . . . . . . . . 51

2-4 The different layers of strained GaAs cathode and the level diagram of the conduction and valence bands of GaAs. . . . . . . . . . . 52

2-5 Schematics of Hall A Møller polarimeter. . . . . . . . . . . . . 54

2-6 Schematics of Hall A Beam Current Monitor. . . . . . . . . . . 56

2-7 Schematics of Hall A Beam Position Monitors. . . . . . . . . . . . 57

2-8 Hall A Arc Measurement. . . . . . . . . . . . . . . 58

2-9 Hall A eP Measurement. . . . . . . . . . . . . . . . 59

2-10 The energy level diagram for ${ }^{85} \mathrm{Rb}$ in an external magnetic field. . . . 62

2-11 A simplified optical pumping process for rubidium. . . . . . . . 63

2-12 Schematics of Hall A polarized ${ }^{3} \mathrm{He}$ target. . . . . . . . . . . 65

2-13 A typical ${ }^{3} \mathrm{He}$ target cell. . . . . . . . . . . . 66

2-14 Hall A target ladder. . . . . . . . . . . . . . . 67

2-15 Principle of Adiabatic Fast Passage (AFP). . . . . . . . . . 69

2-16 Schematics of NMR electronics. . . . . . . . . . . . 71

2-17 Hall A detector package in the electron arm. The detector package in the hadron arm is similar, expect that there is no preshower. . . . . . 74

2-18 Schematics of Hall A VDC wire planes. . . . . . . . . . 75

2-19 A typical trajectory in one of the VDC wire planes. The geodetic is the shortest drift distance and can be used to calculate the perpendicular distance. The intersection point of the particle with the wire plane can be determined with the knowledge of all perpendicular distances from the five fired wires. . . . . . . . . . . . 76

2-20 Schematics of a scintillator plane. There are six scintillator paddles and one phototube is installed at each side of a paddle. . . . . . . 
2-21 Hall A gas Čerenkov detector. . . . . . . . . . . . 78

2-22 Hall A shower detectors. . . . . . . . . . . . 79

2-23 Hall A electron arm trigger electronics. . . . . . . . . . . . 80

2-24 Hall A hadron arm trigger electronics. . . . . . . . . . . 81

2-25 Hall A Data Acquisition System. . . . . . . . . . . 83

3-1 Raw asymmetries for different target polarization angle and beam halfwave plate settings at $\mathrm{Q}^{2}=0.1(\mathrm{GeV} / \mathrm{c})^{2}$. The leftmost point is the elastic asymmetry while the rest are quasielastic asymmetry. . . . . .

3-2 Raw asymmetries for different target polarization angle and beam halfwave plate settings at $\mathrm{Q}^{2}=0.1(\mathrm{GeV} / \mathrm{c})^{2}$, multiplied by their signs. The leftmost point is the elastic asymmetry while the rest are quasielastic asymmetry. The difference between these four settings are mainly due to the change in the product of beam and target polarization from

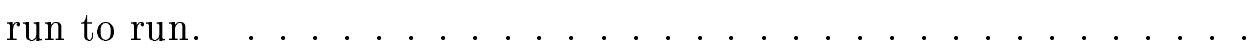

3-3 The ratios of the quasielastic asymmetry to the elastic asymmetry for different target polarization angle and beam half-wave plate settings at $\mathrm{Q}^{2}=0.1(\mathrm{GeV} / \mathrm{c})^{2} \ldots \ldots \ldots \ldots \ldots$

3-4 $\mathrm{N}_{2}$ excitation energy spectra for a typical reference $\mathrm{N}_{2}$ run (above) and a target run (below). The leftmost peak is the $\mathrm{N}_{2}$ elastic peak and the slight shift of the peak position between these two runs is due to the difference in energy loss. The two peaks were not centered at zero excitation energy due to an offset in the spectrometer central momentum setting, which was later corrected. Both elastic peaks were shifted to zero excitation energy before taking their ratio. . . . . . . 96

3-5 The Landau distribution $\phi(\lambda)$ and the integrated probability $\psi(\lambda) \ldots 104$

3-6 Schematic drawing of radiation materials before and after scattering . 107

3-7 Second order Feynman diagrams for internal radiative correction . . . 109

3-8 Integration region over $R$ and $\tau$ is not a rectangle . . . . . . 116 
3-9 Integration region over $v$ and $\tau$ is a rectangle . . . . . . . . 117

3-10 The electron distribution at the entrance of dipole from data and that from a simulation with geometric aperture cuts. The trapezoid shown is the geometric aperture for the dipole entrance . . . . . . . . .

3-11 The electron distribution at the entrance of dipole from data and that from a simulation with physics aperture cuts . . . . . . . . 120

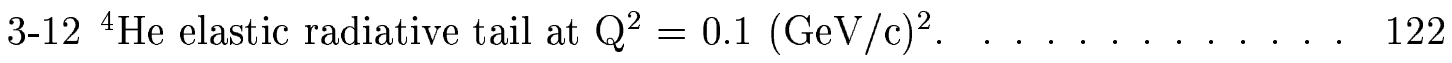

3-13 Comparison between data and simulation for both the elastic and quasielastic spectrum at $\mathrm{Q}^{2}=0.1$ and $0.2(\mathrm{GeV} / \mathrm{c})^{2} \ldots \ldots . . \ldots 123$

3-14 The comparison between experimental cross section and theoretical cross section with full Faddeev calculations at $Q^{2}=0.1$ (above) and 0.2 $(\mathrm{GeV} / \mathrm{c})^{2}$ (below). The statistical error of the data is negligible while the systematic error is due to the uncertainty in the determination of ${ }^{3}$ He target density and spectrometer acceptance. . . . . . . . . . .

4-1 Beam current asymmetry distribution for a typical run from the scaler readouts of all right BCMs. As can be seen, the beam current asymmetry is consistent with zero. . . . . . . . . . . . .

4-2 Beam current asymmetry distribution for the same run from an independent DAQ for the Happex experiment. As can be seen, the beam current asymmetry is consistent with zero. . . . . . . . . 136

4-3 The central beam positions (upper panel) for helicity plus and minus and their difference (lower panel) for some runs at electron $\operatorname{arm} Q^{2}=$ $0.3(\mathrm{GeV} / \mathrm{c})^{2}$, which corresponds to hadron arm $Q^{2}=0.2(\mathrm{GeV} / \mathrm{c})^{2}$.

4-4 Livetime difference between the two beam helicities. The change of sign on the two edges is due to the change of the beam half-wave plate

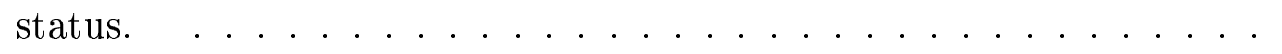

4-5 Difference in scintillator efficiency between the two beam helicities for each paddle and for all runs at $\mathrm{Q}^{2}=0.2(\mathrm{GeV} / \mathrm{c})^{2} \ldots \ldots$ 
4-6 Helicity dependence of the wire efficiency for each wire of the four VDC

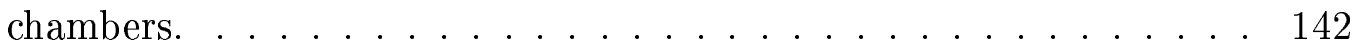

4-7 Variation of the overall empty target dilution factor obtained using different empty target runs. . . . . . . . . . . . . . . . . 144

4-8 Variation of the overall $\mathrm{N}_{2}$ dilution factor obtained using different $\mathrm{N}_{2}$

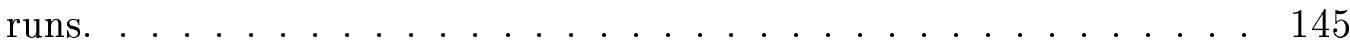

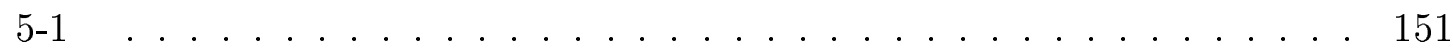

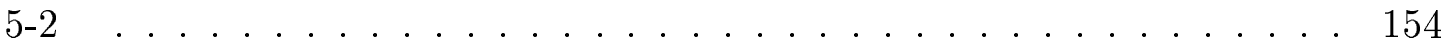

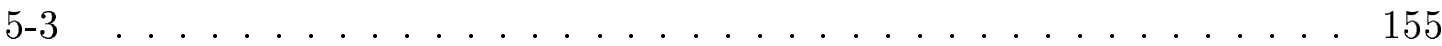

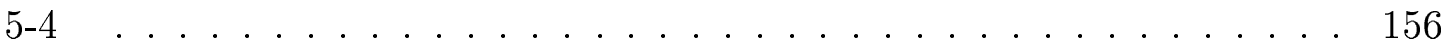

$5-5 \ldots \ldots \ldots \ldots \ldots \ldots \ldots \ldots$

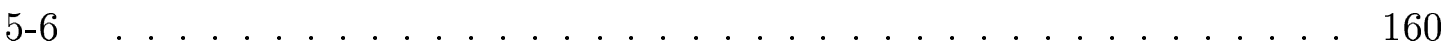

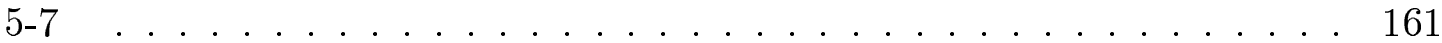

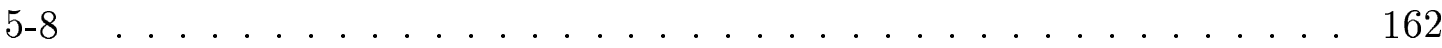

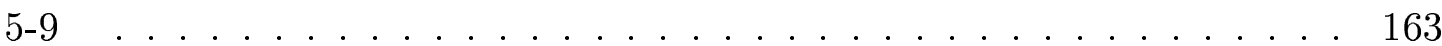

A-1 The class inheritance structure for simulation program Polarized Radiative Correction $(\mathrm{PRC}) . \ldots \ldots \ldots . \ldots \ldots 8$ 


\section{List of Tables}

2.1 Beam polarization results from Møller measurements. . . . . . . . 55

2.2 Hall A High Resolution Spectrometer (HRS) general characteristics. . . . 73

3.1 Empty target yields versus beam positions . . . . . . . . . . 91

3.2 Empty and $\mathrm{N}_{2}$ dilution factors at $\mathrm{Q}^{2}=0.1(\mathrm{GeV} / \mathrm{c})^{2} \ldots \ldots 7$

3.3 Empty and $\mathrm{N}_{2}$ dilution factors at $\mathrm{Q}^{2}=0.2(\mathrm{GeV} / \mathrm{c})^{2} \ldots \ldots$. . . 98

3.4 Empty and $\mathrm{N}_{2}$ false asymmetries at $\mathrm{Q}^{2}=0.1(\mathrm{GeV} / \mathrm{c})^{2} \ldots \ldots$

3.5 Empty and $\mathrm{N}_{2}$ false asymmetries at $\mathrm{Q}^{2}=0.2(\mathrm{GeV} / \mathrm{c})^{2} \ldots \ldots 9$

3.6 Properties of radiation materials before and after scattering . . . . 108

3.7 Elastic radiative tail dilution factors and asymmetry at $\mathrm{Q}^{2}=0.1(\mathrm{GeV} / \mathrm{c})^{2} 124$

3.8 Elastic radiative tail dilution factors and asymmetry at $\mathrm{Q}^{2}=0.2(\mathrm{GeV} / \mathrm{c})^{2} 125$

3.9 Radiative correction to quasielastic asymmetry at $\mathrm{Q}^{2}=0.1(\mathrm{GeV} / \mathrm{c})^{2} \quad 125$

3.10 Radiative correction to quasielastic asymmetry at $\mathrm{Q}^{2}=0.2(\mathrm{GeV} / \mathrm{c})^{2} \quad 126$

3.11 Correction due to bin-averaging and acceptance effects at $\mathrm{Q}^{2}=0.1(\mathrm{GeV} / \mathrm{c})^{2} 127$

3.12 Correction due to bin-averaging and acceptance effects at $\mathrm{Q}^{2}=0.2(\mathrm{GeV} / \mathrm{c})^{2} 127$

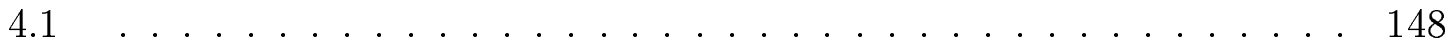

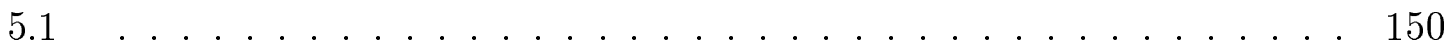

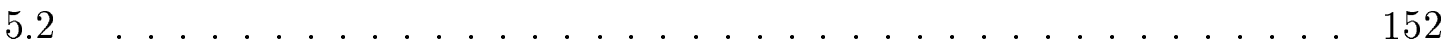




\section{Chapter 1}

\section{Introduction}

\subsection{Physics Motivation}

A thorough understanding of the forces between nucleons, the basic constituents of all nuclei, has been a fundamental goal in nuclear physics for many decades. Realistic models for nucleon-nucleon $(N N)$ interactions have been constructed to fit a large body of $p p$ and $p n$ scattering data accumulated over the past half century. The resulting $N N$ potentials have been applied to the simplest two-nucleon bound state and are able to explain most properties of the deuteron. It is also of great interest to investigate how the nuclear forces are modified in nuclear systems with more than two nucleons. Three-nucleon systems, ${ }^{3} \mathrm{He}$ and ${ }^{3} \mathrm{H}$, are the simplest systems where some of these modifications, in particular three-nucleon forces, start to play a role and thus a careful study of three-nucleon systems, both the bound states and the scattering states, is essential for a complete understanding of the nuclear forces.

With the rapid development of both experimental techniques and computational resources, which are essential for modern few-body theoretical calculations, such a study has become possible. Indeed, in the past decade three-nucleon systems have been an excellent testing ground between theory and experiment in nuclear physics [1]. In the context of electromagnetic processes, exact non-relativistic Faddeev calcula- 
tions for both the ground state and the continuum of ${ }^{3} \mathrm{H}$ and ${ }^{3} \mathrm{He}$ have been carried out using a variety of modern nucleon-nucleon $(N N)$ potentials [2-4]. The exact treatment of final-state interactions (FSI) in the Faddeev calculation results in a much improved description of unpolarized $p d$ capture and breakup channels $[2,4]$, as well as unpolarized electron scattering from three-nucleon systems [3]. This has provided important information on the nuclear ground-state structure and thus allows a deeper understanding of the underlying nuclear force. With the availability of polarized beams and polarized targets, it has become possible to study additional spin-dependent quantities. Polarized ${ }^{3} \mathrm{He}$ is an ideal target for such a study.

Polarized ${ }^{3} \mathrm{He}$ is also important as an effective neutron target $[5,6]$, because its ground state wave function is dominated by the $S$-state in which the proton spins cancel and the nuclear spin is carried entirely by the neutron. The spin-dependent asymmetry is thus sensitive to the neutron electromagnetic form factors in the vicinity of the quasielastic peak of polarized electrons scattering from a polarized ${ }^{3} \mathrm{He}$ target [5-10]. Recently there has been significant progress in extracting neutron electromagnetic form factors from double-polarization electron- ${ }^{3} \mathrm{He}$ quasielastic scattering experiments [11-15]. In recent years, there have also been extensive efforts [16-19] in studying polarized inelastic scattering of electrons from polarized ${ }^{3} \mathrm{He}$ targets in the deep inelastic and resonance regions aiming at understanding the underlying neutron spin structure. The extraction of the neutron spin structure functions from these experiments requires detailed knowledge of the ${ }^{3} \mathrm{He}$ nuclear ground-state structure $[6,20,21]$.

However, to probe the nuclear ground state structure, to extract the neutron electromagnetic form factors or to extract the neutron spin structure function in the resonance region, the reaction mechanism, especially FSI and meson-exchange currents (MEC) effects, must be well understood. Recently, a non-relativistic Faddeev calculation which includes both FSI and MEC has been carried out [4] for the first time, and describes very well the new precision data [12] on the transverse asymme- 
try $A_{T^{\prime}}$ near the top of the quasielastic peak from the ${ }^{3} \overrightarrow{\mathrm{He}}\left(\vec{e}, e^{\prime}\right)$ process at low $Q^{2}$. However, since FSI and MEC effects are relatively small in this region, it is highly desirable to study another region where these two effects are larger to provide a more stringent constraint on the theory. The threshold region of inclusive electron scattering from ${ }^{3} \mathrm{He}$, which extends from the two-body breakup threshold (with breakup energy of $5.5 \mathrm{MeV}$ ), the three-body breakup threshold (with breakup energy of 7.7 $\mathrm{MeV}$ ) to the low energy transfer side of the quasielastic peak, is an ideal place for such a study. First, FSI effects are expected to be large in the threshold region since the final state nucleons have less kinetic energy and thus have a higher probability of interacting with each other. Secondly, it has been shown that a substantial contribution from MEC is needed to describe the measured elastic electromagnetic form factors of three-nucleon systems [22]. Therefore one would expect a large MEC effect in the threshold region as well.

A first systematic measurement of the unpolarized cross sections in the threshold region of inelastic electron scattering from ${ }^{3} \mathrm{He}$ and ${ }^{3} \mathrm{H}$ was carried out in 1994 [23]. The cross sections were measured for excitation energies below $18 \mathrm{MeV}$ and longitudinal and transverse response functions $R_{L}$ and $R_{T}$ were extracted using Rosenbluth separations for a three-momentum transfer from 0.88 to $2.87 \mathrm{fm}^{-1}$. Agreement between data and Faddeev calculations with FSI effects was very good. The inclusion of FSIs was found to be very important, changing the response functions by factors of two or three in some kinematics.

To explore the spin-dependent response functions in the threshold region, a first measurement of the spin-dependent asymmetry in the threshold region of ${ }^{3} \overrightarrow{\mathrm{He}}\left(\vec{e}, e^{\prime}\right)$ was carried out in 1995 [24]. Comparison between data and plane wave impulse approximation showed significant discrepancy, which suggested that a more sophisticated calculation including FSI effects and possibly MEC effects might be necessary. However, the large statistical error in the data prevented further conclusions to be drawn. 
Thus a precision measurement of spin observables in the threshold region of ${ }^{3} \mathrm{He}\left(\vec{e}, e^{\prime}\right)$ is necessary to provide us with more complete information on the reaction mechanism, thereby placing significant constraints on the theoretical uncertainties in probing the ${ }^{3} \mathrm{He}$ ground state structure and in extracting the neutron electromagnetic form factors from electron scattering from ${ }^{3} \mathrm{He}$. In addition, such a high precision measurement would almost surely provide strong motivation for the development of more sophisticated few-body theoretical calculations. In this thesis we report the first precision measurement of the spin-dependent asymmetry in the threshold region of ${ }^{3} \mathrm{He}\left(\vec{e}, e^{\prime}\right)$ and compare the results with state-of-the-art Faddeev calculations which include both FSI and MEC effects.

\section{$1.2 \quad$ Nuclear Interaction}

Traditionally a nucleus is regarded as a system of interacting protons and neutrons. The non-relativistic Hamiltonian of this system can be written as

$$
H=\sum_{i} \frac{\mathbf{p}_{\mathbf{i}}^{2}}{2 m}+\sum_{i<j} v_{i j}+\sum_{i<j<k} V_{i j k}+\cdots
$$

where the nucleons interact via two-, three-, and possibly many-body interactions. This much simplified picture was proposed shortly after the discovery of the neutron in 1932, and after decades of theoretical and experimental work, it was finally justified in a field-theoretical framework with the development of chiral perturbation theory, the low-energy effective theory of QCD [25].

In this section we will describe the dominant part of the nuclear interaction, the nucleon-nucleon $(N N)$ interaction, and some of its natural extensions. We then proceed to discuss an important topic of meson-exchange currents, which follow naturally from the nucleon-nucleon interaction and have been an essential ingredient in explaining many experimental observables in nuclear electroweak processes. 


\subsubsection{Nucleon-Nucleon Potentials}

The $N N$ interaction has been known for a long time to have a very rich structure. Its Hamiltonian involves both the spin operator $\boldsymbol{\sigma}$, which represents the intrinsic angular momentum of the nucleon, and the isospin operator $\tau$, which represents the two charge states of the nucleon, the proton and neutron. It is well-known that the $N N$ interaction has a long-range attractive part and a short-range repulsive part.

The long-range component of the NN interaction is due to the exchange of the lightest meson, the pion, and is described by the Yukawa potential, also called the one-pion exchange potential (OPEP), which can be written as

$$
\begin{aligned}
V^{O P E} & =\frac{f_{\pi N N}^{2}}{4 \pi} \frac{m_{\pi}}{3}\left[Y_{\pi}(r) \boldsymbol{\sigma}_{i} \cdot \boldsymbol{\sigma}_{j}+T_{\pi}(r) S_{i j}\right] \boldsymbol{\tau}_{i} \cdot \boldsymbol{\tau}_{j} \\
Y_{\pi}(r) & =\frac{e^{-\mu r}}{\mu r} \\
T_{\pi}(r) & =\left[1+\frac{3}{\mu r}+\frac{3}{(\mu r)^{2}}\right] Y_{\pi}(r)
\end{aligned}
$$

where $m_{\pi}$ is the mass of the exchanged pion, $\mu$ is the inverse pion mass, $f_{\pi N N}$ is the coupling constant, and

$$
S_{i j}=3 \boldsymbol{\sigma}_{i} \cdot \hat{\mathbf{r}} \boldsymbol{\sigma}_{j} \cdot \hat{\mathbf{r}}-\boldsymbol{\sigma}_{i} \cdot \boldsymbol{\sigma}_{j}
$$

is the tensor operator. The OPEP is clearly spin dependent through the $\boldsymbol{\sigma}_{i} \cdot \boldsymbol{\sigma}_{j}$ term and the tensor operator, and the spatial and spin degrees of freedom are strongly correlated in nuclear systems also due to the tensor operator.

At intermediate and short distances, the $N N$ interaction is much more complicated. However, the large body of $p p$ and $p n$ scattering data accumulated over the past half century provides very strong constraints on the form of the interaction. Five modern $N N$ potentials have been constructed by fitting to the experimental database. These include the Argonne $v_{18}$ (AV18) potential, the CD-Bonn potential, and three Nijmegen potentials (Nijm I, Nijm II and Reid 93). Each model fits the $N N$ database extremely well, with a $\chi^{2} /$ datum of nearly one. 
The AV18 potential [26] contains an electromagnetic interaction and a phenomenological short- and intermediate-range component, in addition to the long range OPEP. The electromagnetic component includes the magnetic moment interaction and onephoton exchange Coulomb interaction, among others. The short- and intermediaterange component is parameterized in the form

$$
V_{S T}^{R}=v_{S T}^{c}(r)+v_{S T}^{l 2}(r) \mathbf{L}^{2}+v_{S T}^{t}(r) S_{i j}+v_{S T}^{l s}(r) \mathbf{L} \cdot \mathbf{S}+v_{S T}^{l s 2}(r)(\mathbf{L} \cdot \mathbf{S})^{2}
$$

The radial functions all have the form

$$
v_{S T}^{i}=I_{S T}^{i} T^{2}(r)+\left[P_{S T}^{i}+\mu r Q_{S T}^{i}+(\mu r)^{2} R_{S T}^{1}\right] W(r)
$$

where $I_{S T}^{i}, P_{S T}^{i}, Q_{S T}^{i}$, and $R_{S T}^{1}$ are parameters obtained from fit. $T(r)$ is the tensor Yukawa function with a range parameter $\alpha$

$$
T(r)=\left(1+\frac{3}{\mu r}+\frac{3}{(\mu r)^{2}}\right) \frac{e^{-\mu r}}{\mu r}\left(1-e^{-\alpha r^{2}}\right)^{2}
$$

and $W(r)$ is the Woods-Saxon function

$$
W(r)=\frac{1}{1+e^{\left(r-r_{0}\right) / a}}
$$

where $r_{0}$ and $a$ are shape parameters also obtained from fit. The AV18 potential is maximally local, since it contains at most terms proportional to $\mathbf{L}^{2}$.

The CD-Bonn potential [27] was constructed based on field theoretical perturbation theory. The lowest order contributions to the $N N$ interaction are the one-boson exchange diagrams, which include all mesons with masses below the nucleon mass, i.e., $\pi, \eta, \rho$ and $\omega$. However $\eta$ is dropped because its coupling constant to the nucleon is very small. There are also higher order multimeson exchanges, such as $2 \pi$ exchange and $\pi \rho$ exchange, which are approximated by introducing two scalar-isoscaler $\sigma$ bosons. Thus the CD-Bonn potential is completely defined in terms of one-boson 
exchange, and the Feynman diagrams are shown in Figure 1-1. The CD-Bonn potential is the only fully nonlocal, or momentum-dependent, potential among modern potentials, due to its field theoretical approach. This is consistent with the nonlocal character of the nuclear force. In fact, the composite structure of hadrons should lead to large nonlocalities at short range [28].

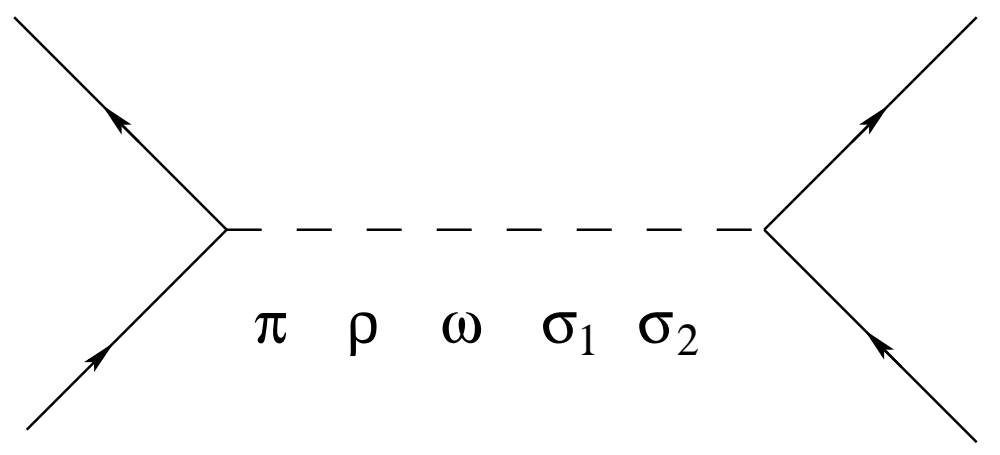

Figure 1-1: One-boson exchange Feynman diagrams that define the CD-Bonn NN potential.

The Nijmegen potentials [29] include traditional long range OPEP and use exchanges of heavy mesons such as $\rho, \omega, a_{0}$ and $f_{0}$ for the short range part of the interaction. The Nijm I potential contains certain nonlocal terms, which are removed in the Nijm II potential.

\subsubsection{Three-Nucleon Forces and Relativistic Effects}

All the local $N N$ potentials give nearly identical results for the triton binding energy: $7.62 \pm 0.01 \mathrm{MeV}$, as compared to the experimental value of $8.48 \mathrm{MeV}$. Thus it is clear that local two-body potentials are not sufficient to reproduce the three-nucleon binding energy. Two natural extensions to two-nucleon interactions are three-nucleon interactions (3NFs) and relativistic corrections.

The earliest three-nucleon force, proposed in 1957 by Fujita and Miyazawa [30], corresponds to single-pion exchanges between three nucleons with the intermediate 
excitation of a $\Delta$-isobar resonance, as shown in Figure 1-2. The interacting potential can be written as

$$
V_{i j k}^{2 \pi}=A_{2 \pi}\left[\left\{X_{i j}, X_{i k}\right\}\left\{\boldsymbol{\tau}_{i} \cdot \boldsymbol{\tau}_{j}, \boldsymbol{\tau}_{i} \cdot \boldsymbol{\tau}_{k}\right\}+\frac{1}{4}\left[X_{i j}, X_{i k}\right]\left[\boldsymbol{\tau}_{i} \cdot \boldsymbol{\tau}_{j}, \boldsymbol{\tau}_{i} \cdot \boldsymbol{\tau}_{k}\right]\right]
$$

where

$$
X_{i j}=Y_{\pi}(r) \boldsymbol{\sigma}_{i} \cdot \boldsymbol{\sigma}_{j}+T_{\pi}(r) S_{i j}
$$

and $\{A, B\}$ and $[A, B]$ represent anticommutators and commutators of two operators $A$ and $B$.

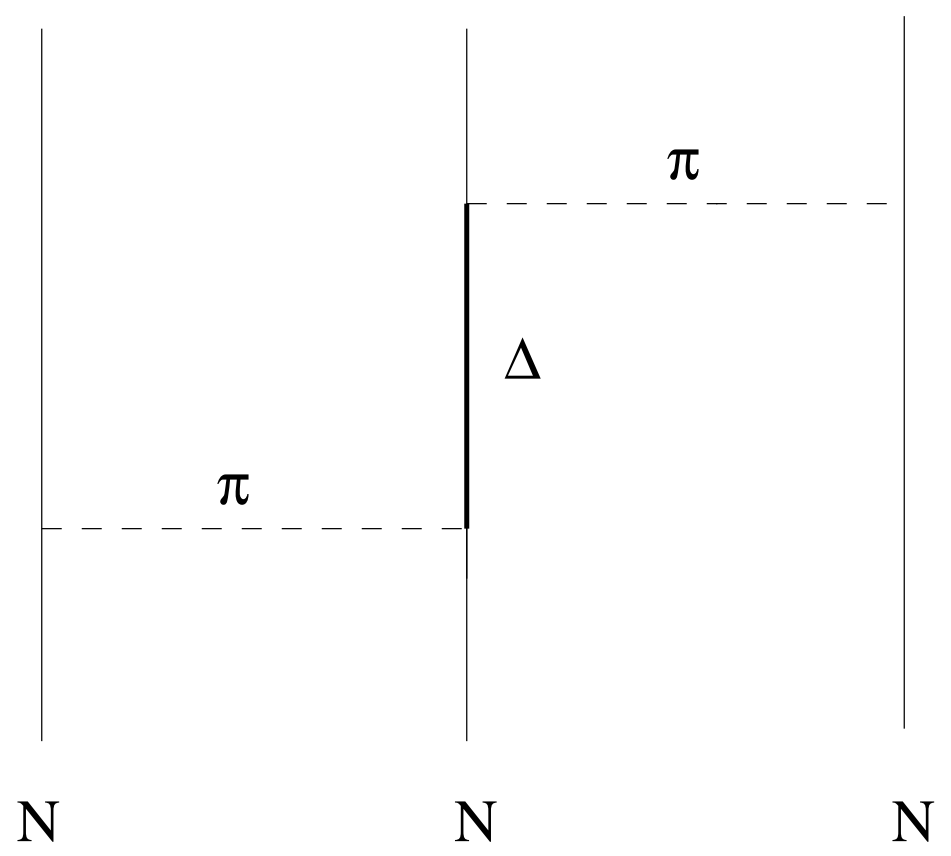

Figure 1-2: Fujita-Miyazawa three-nucleon force through the exchange of two pions between three nucleons, during which one of the nucleons is excited to a $\Delta$ resonance.

One of the most widely used modern three-nucleon forces is the Urbana IX (UIX) [31], which contains a Fujita-Miyazawa term $V_{i j k}^{2 \pi}$ and a phenomenological short-range repulsive term $V_{i j k}^{R}$ :

$$
V_{i j k}=V_{i j k}^{2 \pi}+V_{i j k}^{R}
$$


with

$$
V_{i j k}^{R}=U_{0} \sum_{c y c} T_{\pi}^{2}\left(r_{i j}\right) T_{\pi}^{2}\left(r_{i k}\right)
$$

The constants $A_{2 \pi}$ and $U_{0}$ are adjusted to reproduce the triton binding energy.

Other modern 3NFs include the Brazil force [32], the Tucson-Melbourne force [33] and the Texas-Los Alamos force [34]. The Brazil force contains the Fujita-Miyazawa term and adds some chiral corrections. The Tucson-Melbourne force also contains the Fujita-Miyazawa term and includes current algebra constraints. The Texas-Los Alamos force is entirely motivated by chiral perturbation theory.

A wide variety of relativistic calculations of light nuclei bound state properties have been carried out by extending the one-boson-exchange mechanism to relativistic treatments. Moderate success has been achieved. For example, using the relativistic one-boson-exchange formalism, the triton binding energy was calculated to be 8.19 $\mathrm{MeV}$ [27], which is much closer to the experimental value.

It is also possible to perform calculations within a $v / c$ expansion scheme. One class of relativistic corrections within this scheme is purely kinematics, i.e., replacing the nonrelativistic kinetic energy with the corresponding relativistic expression and including a momentum-dependence in the two- and three-nucleon interactions in the Hamiltonian [35]

$$
H=\sum_{i} \sqrt{\mathbf{p}_{i}^{2}+m^{2}}+\sum_{i<j} v_{i j}\left(\mathbf{r}_{i j}, \mathbf{P}_{i j}\right)+\sum_{i<j<k} V_{i j k}\left(\mathbf{r}_{i j}, \mathbf{r}_{i k} \mathbf{P}_{i j k}\right)
$$

where $\mathbf{P}_{i j}$ and $\mathbf{P}_{i j k}$ are the total momentum of the two- and three-body subsystems.

However, fully relativistic calculations within a relativistic Hamiltonian formalism are not yet well developed. 


\subsubsection{Meson-Exchange Currents}

The nuclear electromagnetic current operators are needed to describe the electromagnetic processes involving nuclei, such as electron scattering from ${ }^{3} \mathrm{He}$ and protonneutron radiative capture. In the so-called impulse approximation (IA), the nuclear electromagnetic current operators are expressed in terms of those associated with individual protons and neutrons, and thus are all one-body operators. However, such a description is certainly incomplete and the existence of additional meson-exchange current operators follows naturally from the fact that the nucleon-nucleon interaction is mediated by pion exchanges at large distances and other meson-exchange mechanisms at short and intermediate distances. In essence, the meson-exchange currents are simply effective two- and many-body current operators arising from the elimination of the mesonic degrees of freedom from the nuclear state vector. Thus this picture is only valid at energies below the threshold for meson production, since above this threshold the mesonic degrees of freedom have to be explicitly included in the nuclear state vector.

To see clearly how two- and many-body meson-exchange current operators follow naturally from the nucleon-nucleon interaction, let us expand the nuclear electromagnetic current operators, namely the charge $(\rho(\mathbf{q}))$ and current $(\mathbf{j}(\mathbf{q}))$ operators, into a sum of one-, two-, and many-body terms that operate on the nucleonic degrees of freedom:

$$
\begin{aligned}
& \rho(\mathbf{q})=\sum_{i} \rho_{i}^{(1)}(\mathbf{q})+\sum_{i<j} \rho_{i j}^{(2)}(\mathbf{q})+\cdots \\
& \mathbf{j}(\mathbf{q})=\sum_{i} \mathbf{j}_{i}^{(1)}(\mathbf{q})+\sum_{i<j} \mathbf{j}_{i j}^{(2)}(\mathbf{q})+\cdots
\end{aligned}
$$

where the one-body operators $\rho_{i}^{(1)}(\mathbf{q})$ and $\mathbf{j}_{i}^{(1)}(\mathbf{q})$ are obtained from the covariant 
single-nucleon current [36]

$$
j^{\mu}=\bar{u}\left(\mathbf{p}^{\prime}\right)\left[F_{1}\left(Q^{2}\right) \gamma^{\mu}+F_{2}\left(Q^{2}\right) \frac{i \sigma^{\mu \nu} q_{v}}{2 m}\right] u(\mathbf{p})
$$

where $\mathbf{p}$ and $\mathbf{p}^{\prime}$ are the initial and final momenta of a nucleon of mass $\mathrm{m}$, and $F_{1}\left(Q^{2}\right)$ and $F_{2}\left(Q^{2}\right)$ are its Dirac and Pauli form factors as a function of the minus fourmomentum transfer squared $Q^{2}$.

The electromagnetic current operators must satisfy the continuity equation

$$
\mathbf{q} \cdot \mathbf{j}(\mathbf{q})=[H, \rho(\mathbf{q})]
$$

where the Hamiltonian $H$ includes two- and three- nucleon interactions

$$
H=\sum_{i} \frac{\mathbf{p}_{\mathbf{i}}^{\mathbf{2}}}{2 m}+\sum_{i<j} v_{i j}+\sum_{i<j<k} V_{i j k}
$$

To lowest order in $1 / m$, the continuity equation separates into separate equations for the one-, two- and many-body current operators [35]:

$$
\begin{aligned}
\mathbf{q} \cdot \mathbf{j}_{i}^{(1)}(\mathbf{q}) & =\left[\frac{\mathbf{p}_{i}^{2}}{2 m}, \rho_{i, N R}^{(1)}(\mathbf{q})\right] \\
\mathbf{q} \cdot \mathbf{j}_{i j}^{(2)}(\mathbf{q}) & =\left[v_{i j}, \rho_{i, N R}^{(1)}(\mathbf{q})+\rho_{j, N R}^{(1)}(\mathbf{q})\right]
\end{aligned}
$$

and higher order terms. Note that we use $\rho_{i, N R}^{(1)}(\mathbf{q})$ because $\rho_{i}^{(1)}(\mathbf{q})$ also contains relativistic corrections which are of orders $1 / m^{2}$ and higher. In the following we focus on two-body meson-exchange currents.

The most important two-body currents are constructed from the nucleon-nucleon interaction and are constrained by the continuity equation. Thus they do not contain any free parameters and are essentially model-independent. For example, in the CD-Bonn potential, at intermediate and large distances the nuclear interaction is mediated by $\pi$-meson and $\rho$-meson exchanges, as shown in Figure 1-3. The cor- 
responding isovector two-body currents can be obtained from the $\pi N N$ and $\rho N N$ coupling Langrangians following the Riska prescription [37]. At short distances the nucleon-nucleon interaction leads to $\sigma$-like and $\omega$-like meson-exchange currents for the isospin-independent terms and $\rho$-like meson-exchange currents for the isospindependent terms [38].

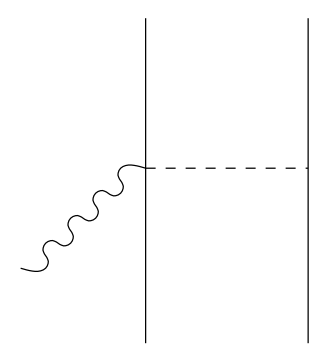

(a)

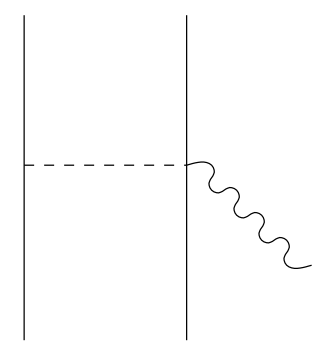

(b)

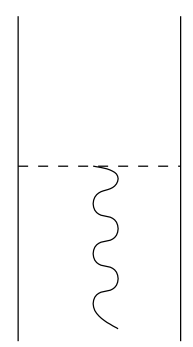

(c)

Figure 1-3: Feynman diagram representation of the isovector currents associated with $\pi$ and $\rho$ exchanges. Solid lines represent nucleons, dashed lines represent $\pi$ or $\rho$ mesons and wavy lines represent photons.

In addition, there are also model-dependent two-body currents which are purely transverse and thus not constrained by the continuity equation. One class of modeldependent currents that has been commonly considered in the literature is associated with electromagnetic transition couplings between different mesons, for example, the $\rho \pi \gamma$ and $\omega \pi \gamma$ mechanisms as shown in Figure 1-4. The associated two-body currents have short range due to the large masses of $\rho$ - and $\omega$-mesons $[39,40]$. A second class of model-dependent currents is associated with the $\Delta$-isobar degrees of freedom. In the current theoretical framework we view the nucleus as made up of nucleons and assume that all other subnucleonic degrees of freedom can be eliminated in favor of effective two- and many-body operators acting on the nucleon's coordinates. As a first order correction to this picture, we can take the lowest excitation of the nucleon, the $\Delta$ isobar, as additional constituents of the nucleus [41,42]. With the inclusion of $\Delta$ admixtures in the nuclear wave function, we need to consider interactions involving 
both nucleons and the $\Delta$ isobars, such as $N N \rightarrow N \Delta$ and $N N \rightarrow \Delta \Delta$ transition interactions, the Feynman diagrams of which are shown in Figure 1-5. Additional electromagnetic currents can then be constructed from these interactions [35].

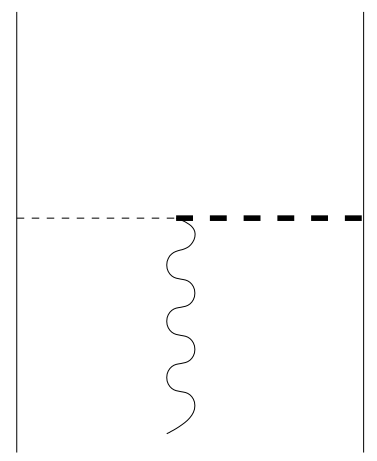

Figure 1-4: Feynman diagram representation of the isoscaler $\rho \pi \gamma$ and isovector $\omega \pi \gamma$ transition currents. Solid lines represent nucleons, dashed lines represent pions while thick-dashed lines vector mesons $\rho$ or $\omega$, and wavy lines represent photons.

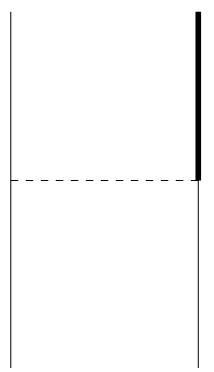

(a)

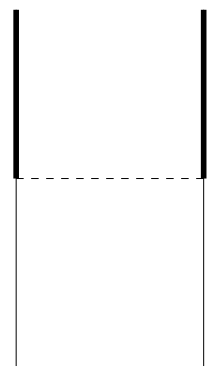

(b)

Figure 1-5: Feynman diagram representation of the $N N \rightarrow N \Delta$ and $N N \rightarrow \Delta \Delta$ transition interactions. Solid lines represent nucleons, thick-solid lines represent $\Delta$ isobars, and wavy lines represent photons.

\subsection{Spin-Dependent Asymmetry in Quasielastic ${ }^{3} \overrightarrow{\mathrm{He}}\left(\vec{e}, e^{\prime}\right)$}

The spin-dependent asymmetry in quasielastic scattering of longitudinally polarized electrons from polarized ${ }^{3} \mathrm{He}$ nuclei was first calculated in a plane wave impulse ap- 
proximation (PWIA) within the closure approximation, where a spin-dependent momentum distribution was employed to describe the nuclear effects [5]. Later, a PWIA calculation was carried out using a spin-dependent spectral function, which summarizes all of the nuclear structure information in the process [8]. One major drawback of a PWIA calculation is that it does not take the interactions between the final-state nucleons into account. There are quite a few techniques available to include the FSI effects. One is the Green's-function Monte Carlo which relies upon the path-integral approach to evaluate the imaginary-time propagation of the wave function [43]. However, as for any Monte Carlo method, there is inherent statistic error in this method. The second technique, called Faddeev methods, can take FSI exactly into account for any type of realistic NN force. In addition, it is also straightforward to include meson exchange currents (MECs) in Faddeev calculations.

We will first derive the general formalism for inclusive electron scattering from a nucleus, in particular the spin-dependent asymmetry in quasielastic ${ }^{3} \mathrm{He}\left(\vec{e}, e^{\prime}\right)$ process, then describe the PWIA calculations and non-relativistic Faddeev calculations which include both FSI and MEC effects in detail.

\subsubsection{General Formalism for Inclusive Electron Scattering}

Before presenting the results for quasielastic ${ }^{3} \mathrm{He}\left(\vec{e}, e^{\prime}\right)$, it is useful to examine the general polarized $\left(e, e^{\prime}\right)$ process. For such an inclusive experiment, the final nuclear state is not observed. A general $\left(e, e^{\prime}\right)$ spectrum is shown in Figure 1-6, where the cross section is plotted as a function of the energy transfer $\omega$ for a fixed minus fourmomentum transfer squared $Q^{2}$.

The first sharp peak is due to electron elastic scattering from the nucleus as a whole while the next few sharp peaks correspond to nuclear excitation to discrete states. Note that in real experiments these peaks are much smoother because of radiative effects. Immediately following is a broad peak called the "Giant Resonance", which is due to the excitation of collective modes in the nucleus. Then there is a 
Elastic

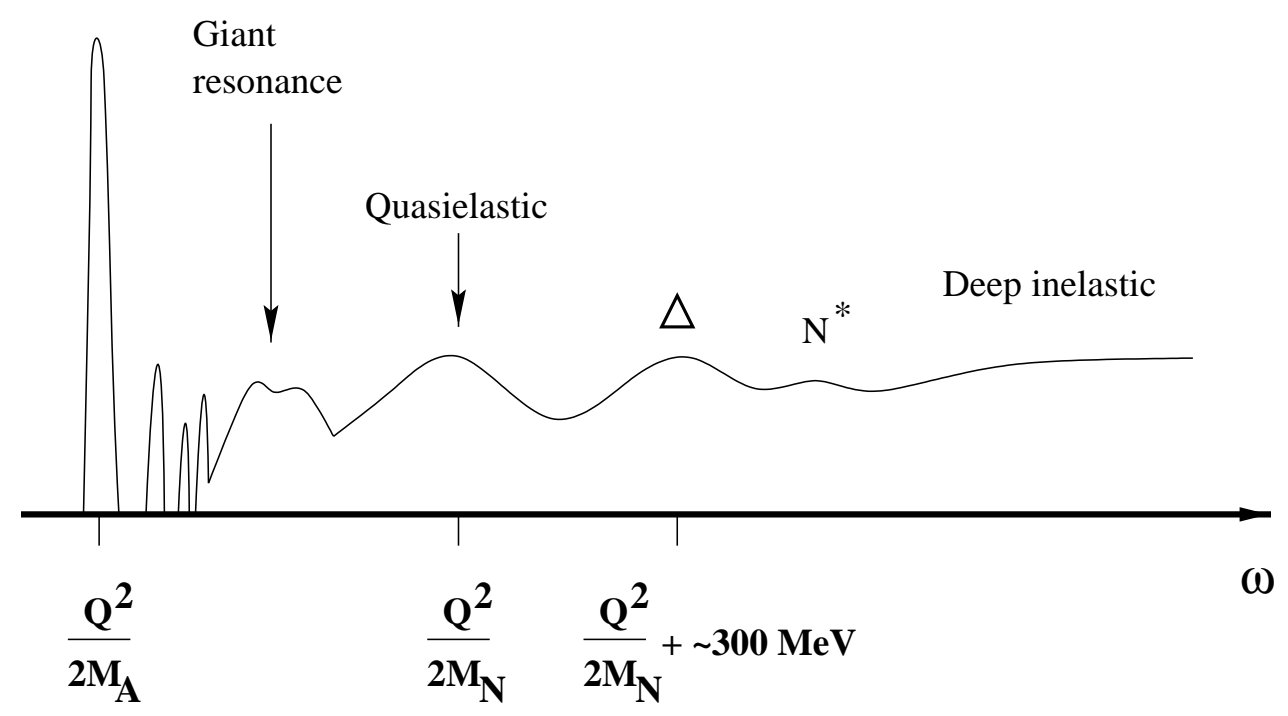

Figure 1-6: A general $\left(e, e^{\prime}\right)$ spectrum.

very broad bump peaked at $\omega \approx Q^{2} / 2 M_{N}$, which is called the quasielastic peak and corresponds to the electron being elastically scattered from a single nucleon inside the nucleus. The next few bumps correspond to the excitation of a nucleon to $\Delta$ and $N^{*}$ states. Finally comes a relatively flat region called the deep inelastic scattering region, where the nucleon resonances are broad, overlapping and thus not distinguished as bumps. In this region, the electron may be thought of as being scattered from individual constituent quarks of the nucleon. For electron scattering from ${ }^{3} \mathrm{He}$, there are no discrete nuclear excited states and giant resonance, so the quasielastic peak immediately follows the elastic peak, and we are only interested in these two channels in this work.

Consider the inclusive scattering of a longitudinally polarized electron with fourmomentum $k=(E, \mathbf{k})$ from a spin- $\frac{1}{2}$ nucleus with four-momentum $P_{A}=\left(M_{A}, 0\right)$ and polarization four-vector $S_{A}$. The scattered electron is detected and thus its fourmomentum $k^{\prime}=\left(E^{\prime}, \mathbf{k}^{\prime}\right)$ is known, while the final hadronic matter $\mathrm{X}$ is not observed. Denote $q=k-k^{\prime}$ as the four-momentum transfer and $Q^{2}=-q^{2}$. There are two 
kinds of interactions between the electron and the nucleus, the electromagnetic interaction via virtual photon exchange and the weak interaction through the exchange of massive $Z^{0}$ boson. However, since the intermediate energy scale is much smaller than the mass of the $Z^{0}$ boson, the electromagnetic interaction dominates the weak interaction by many orders of magnitude. Also, we will assume only one virtual photon is exchanged since $Z \alpha \ll 1$, where $Z$ is the number of protons inside the nucleus and $\alpha \approx 1 / 137$ is the fine structure constant. The Feynman diagram in the first-order Born approximation is shown in Figure 1-7.

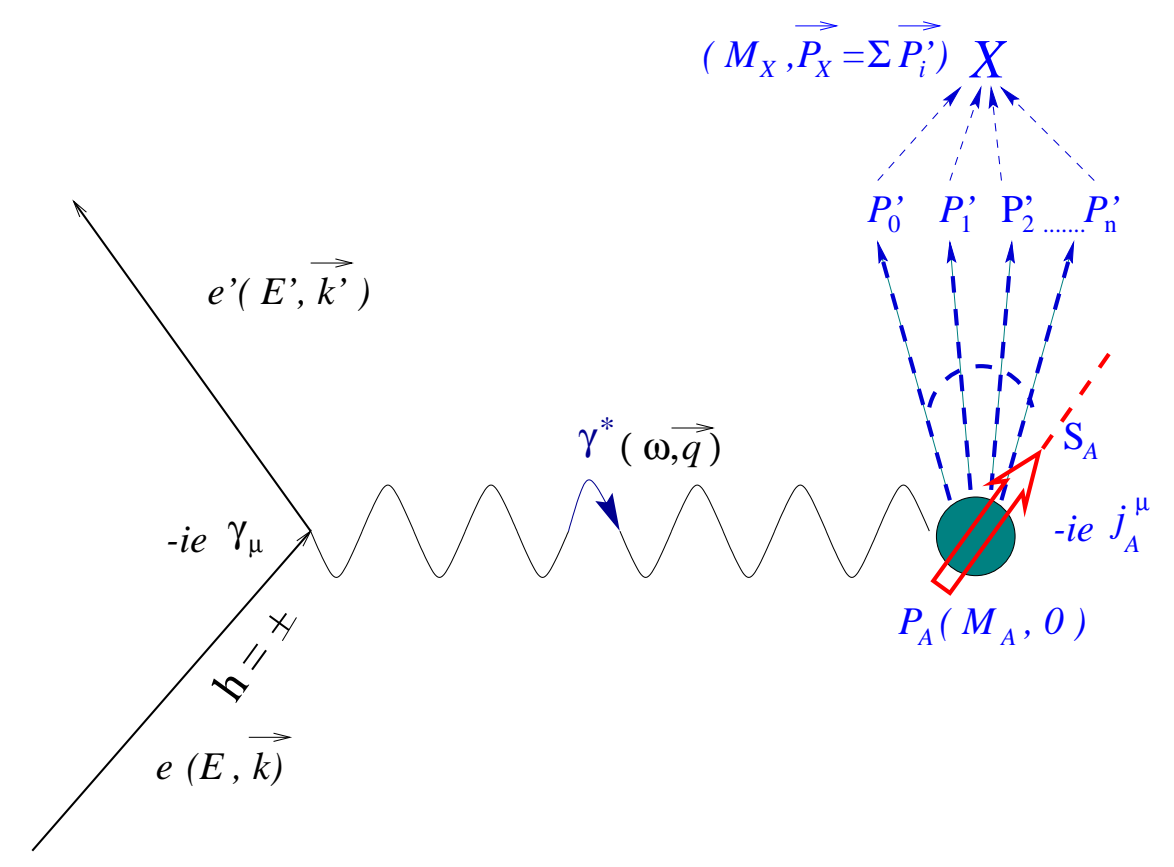

Figure 1-7: The Feynman diagram for the first-order Born approximation.

The inclusive cross section can be written as [44]

$$
\frac{d \sigma}{d \Omega d E^{\prime}}=\frac{4 \alpha^{2}}{Q^{4}} \frac{E^{\prime}}{E} L_{\mu \nu} W^{\mu \nu}
$$


where the leptonic tensor is given by

$$
L_{\mu \nu}=k_{\mu}^{\prime} k_{\nu}+k_{\nu}^{\prime} k_{\mu}-g_{\mu \nu} k^{\prime} \cdot k+i m_{e} \varepsilon_{\mu \nu \alpha \beta} s^{\alpha} q^{\beta}
$$

and the hadronic tensor for a spin- $\frac{1}{2}$ nucleus is given by

$$
\begin{aligned}
W^{\mu \nu}= & \left(-g^{\mu \nu}+\frac{q^{\mu} q^{\nu}}{q^{2}}\right) W_{1}+\tilde{P}_{A}^{\mu} \tilde{P}_{A}^{\nu} \frac{W_{2}}{M_{A}^{2}} \\
& +i \varepsilon_{\mu \nu \alpha \beta} q_{\alpha}\left[S_{A \beta} \frac{G_{1}}{M_{A}}+\left[\left(q \cdot P_{A}\right) S_{A \beta}-\left(q . S_{A}\right) P_{A \beta}\right] \frac{G_{2}}{M_{A}^{3}}\right]
\end{aligned}
$$

where $W_{1}$ and $W_{2}$ are the spin-averaged structure functions while $G_{1}$ and $G_{2}$ are the spin-dependent ones, and

$$
\tilde{P}_{A}=P_{A}-\frac{q \cdot P_{A}}{q^{2}} q
$$

In the center-of-mass system the structure functions are related to the frameindependent nuclear response functions, namely the spin-averaged transverse and longitudinal ones $R_{T}$ and $R_{L}$, and the spin-dependent transverse and mixed transverselongitudinal ones $R_{T^{\prime}}$ and $R_{T L^{\prime}}[45]$

$$
\begin{aligned}
W_{1} & =\frac{1}{2} R_{T} \\
W_{2} & =\frac{Q^{4}}{\mathbf{q}^{4}} R_{L}+\frac{1}{2} \frac{Q^{2}}{\mathbf{q}^{2}} R_{T} \\
G_{1} & =-\frac{1}{2} \frac{M_{A}^{2}}{\mathbf{q}^{2}}\left[\frac{Q^{2}}{M_{A}|\mathbf{q}|} \sqrt{\frac{1}{2}} R_{T L^{\prime}}-\frac{\omega}{M_{A}} R_{T^{\prime}}\right] \\
G_{2} & =-\frac{1}{2} \frac{M_{A}^{2}}{\mathbf{q}^{2}}\left[\frac{\omega}{|\mathbf{q}|} \sqrt{\frac{1}{2}} R_{T L^{\prime}}+R_{T^{\prime}}\right]
\end{aligned}
$$

The final cross section in terms of the response functions can be written as

$$
\frac{d \sigma}{d \Omega d E^{\prime}}=\frac{\alpha^{2} \cos ^{2} \frac{\theta}{2}}{4 E^{2} \sin ^{4} \frac{\theta}{2}}\left\{v_{L} R_{L}\left(Q^{2}, \omega\right)+v_{T} R_{T}\left(Q^{2}, \omega\right)\right.
$$




$$
\left.-h\left[v_{T^{\prime}} \cos \theta^{*} R_{T^{\prime}}\left(Q^{2}, \omega\right)+2 v_{T L^{\prime}} \sin \theta^{*} \cos \phi^{*} R_{T L^{\prime}}\left(Q^{2}, \omega\right)\right]\right\}
$$

where $\theta^{*}$ and $\phi^{*}$ are the polar and azimuthal angles of the target spin direction with respect to the three-momentum transfer $\vec{q}$, as is shown in Figure 1-8.

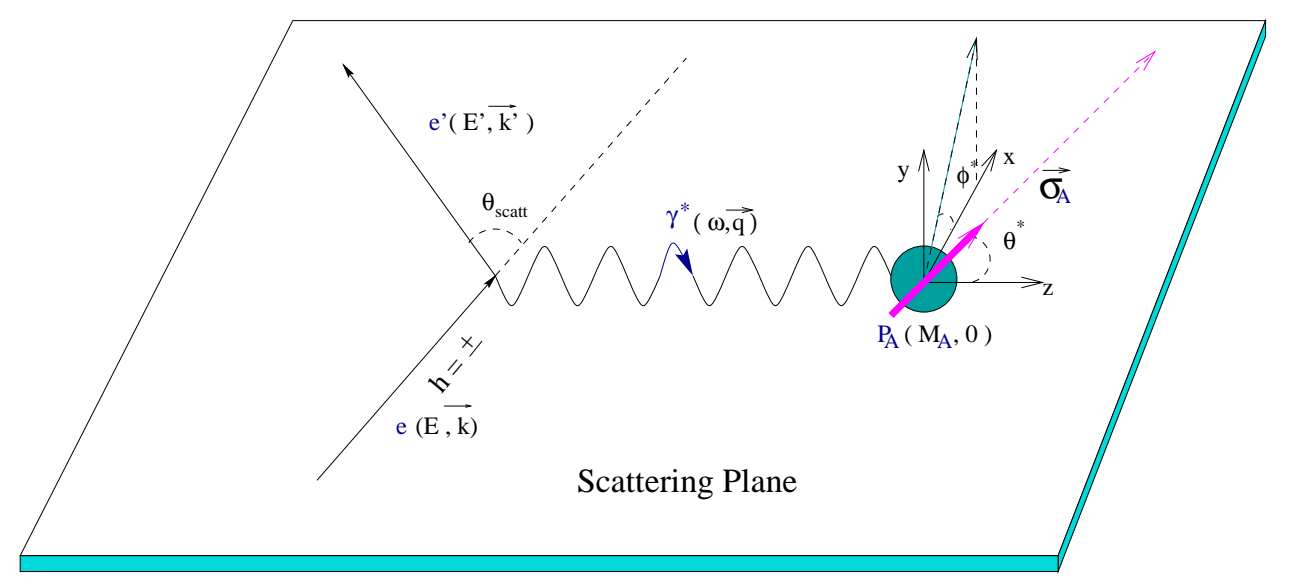

Figure 1-8: The scattering plane.

The $v_{k}$ are kinematic variables defined as

$$
\begin{aligned}
v_{L} & =\frac{Q^{4}}{|\mathbf{q}|^{4}} \\
v_{T} & =\frac{1}{2} \frac{Q^{2}}{|\mathbf{q}|^{2}}+\tan ^{2} \frac{\theta}{2} \\
v_{T^{\prime}} & =\tan \frac{\theta}{2} \sqrt{\frac{Q^{2}}{|\mathbf{q}|^{2}}+\tan ^{2} \frac{\theta}{2}} \\
v_{T L^{\prime}} & =-\frac{1}{\sqrt{2}} \frac{Q^{2}}{|\mathbf{q}|^{2}} \tan \frac{\theta}{2}
\end{aligned}
$$

where $\theta$ is the scattering angle.

The spin-dependent quasielastic asymmetry, defined as $A=\frac{\sigma^{h+}-\sigma^{h-}}{\sigma^{h+}+\sigma^{h-}}$, is given in terms of the response functions as

$$
A=-\frac{\cos \theta^{*} \nu_{T^{\prime}} R_{T^{\prime}}+2 \sin \theta^{*} \cos \phi^{*} \nu_{T L^{\prime}} R_{T L^{\prime}}}{\nu_{L} R_{L}+\nu_{T} R_{T}}
$$


By choosing $\theta^{*}=0^{\circ}\left(90^{\circ}\right)$, one selects the transverse asymmetry $A_{T^{\prime}}$ (longitudinaltransverse asymmetry $\left.A_{T L^{\prime}}\right)$.

The nuclear structure functions or equivalently, the response functions, for quasielastic processes have been obtained through both PWIA calculations and full Faddeev calculations.

\subsubsection{PWIA Calculations}

The simplest description of the quasielastic process is the Plane Wave Impulse Approximation (PWIA), where the virtual photon is totally absorbed by one of the nucleons, which comes out without further interaction with the residual nucleus and thus can be treated as a plane wave. The first-order Feynman diagram for this process is shown in Figure 1-9.

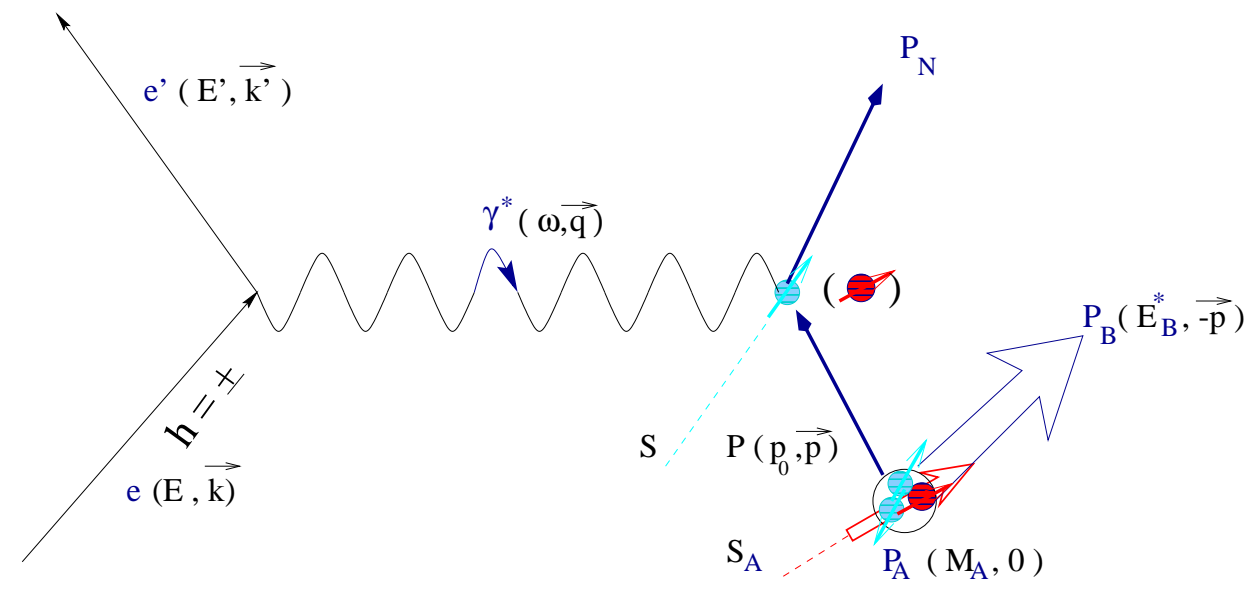

Figure 1-9: The Feynman diagram in Plane Wave Impulse Approximation.

Within the framework of PWIA, the nuclear current tensor is calculated as an operator in nuclear spin space [8]

$$
\left\langle s_{A}^{\prime}\left|W_{A}^{\mu \nu}\left(q, P_{A}\right)\right| s_{A}\right\rangle=\sum_{t_{N}} \sum_{s_{N} s_{N}^{\prime}} \int d^{3} p_{N} \frac{m_{N}}{p_{N}^{0}} \int d E\left\langle s_{N}^{\prime}\left|W_{N t_{N}}^{\mu \nu}\left(q_{N}, p_{N}\right)\right| s_{N}\right\rangle
$$




$$
\times\left\langle s_{N} s_{A}^{\prime}\left|S\left(\mathbf{p}_{N} E t_{N}\right)\right| s_{N}^{\prime} s_{A}\right\rangle
$$

where $p_{N}=\left(\sqrt{M_{N}^{2}+\mathbf{p}_{N}^{2}}, \mathbf{p}_{N}\right)$ is the four-momentum of the struck nucleon, $q_{N}$ is the four-momentum transfer to the nucleon from the electron $\left(Q_{N}^{2}=-q_{N}^{2}\right.$ being positive), $s_{N}\left(t_{N}\right)$ is the nucleonic spin (isospin) projection, and $E$ is the so-called

separation energy. $W_{N\left(t_{N}\right)}^{\mu \nu}\left(q_{N}, p_{N}\right)$ is the nucleonic current tensor and $S\left(\mathbf{p}_{N}, E, t_{N}\right)$ is the spectral function which contains the nuclear structure information. The spinindependent part of $S\left(\mathbf{p}_{N}, E, t_{N}\right)$ is identical to the usual spectral function described in [46] and is interpreted as the probability of finding a nucleon of isospin $t_{N}$ and momentum $\mathbf{p}_{N}$ in the target nucleus $A$, with the residual $(A-1)$ nucleus having an excitation energy determined by the separation energy $E$. Both the nucleonic current tensor $W_{N\left(t_{N}\right)}^{\mu \nu}\left(q_{N}, p_{N}\right)$ and the spectral function $S\left(\mathbf{p}_{N}, E, t_{N}\right)$ are considered operators in spin space and were derived in [8].

\section{The Nucleonic Current Tensor}

The nucleonic current tensor has the following form [8]

$$
\begin{aligned}
W_{N\left(t_{N}\right)}^{\mu \nu}\left(q_{N}, p_{N}\right)= & -\left[\frac{q_{N}^{\mu} q_{N}^{\nu}}{Q_{N}^{2}}+g^{\mu \nu}\right] W_{1}^{N\left(t_{N}\right)}\left(Q_{N}^{2}, \frac{q_{N} \cdot p_{N}}{M_{N}}\right) \\
& +{\tilde{p_{N}}}^{\mu}{\tilde{p_{N}}}^{\nu} \frac{W_{2}^{N\left(t_{N}\right)}\left(Q_{N}^{2}, \frac{q_{N} \cdot p_{N}}{M_{N}}\right)}{M_{N}^{2}} \\
& +i \epsilon^{\mu \nu \alpha \beta} q_{N} \alpha\left[s_{\beta}\left(\boldsymbol{\sigma}_{N}\right) \frac{G_{1}^{N\left(t_{N}\right)}\left(Q_{N}^{2}, \frac{q_{N} \cdot p_{N}}{M_{N}}\right)}{M_{N}}+\left\{q_{N} \cdot p_{N} s_{\beta}\left(\boldsymbol{\sigma}_{N}\right)\right.\right. \\
& \left.\left.-q_{N} \cdot s_{\beta}\left(\boldsymbol{\sigma}_{N}\right) p_{N \beta}\right\} \frac{G_{2}^{N\left(t_{N}\right)}\left(Q_{N}^{2}, \frac{q_{N} \cdot p_{N}}{M_{N}}\right)}{M_{N}^{3}}\right]
\end{aligned}
$$

with

$$
\begin{aligned}
\tilde{p}_{N} & =p_{N}+\frac{q_{N} \cdot p_{N}}{Q_{N}^{2}} q_{N} \\
s\left(\boldsymbol{\sigma}_{N}\right) & =\left[\frac{\mathbf{p}_{N} \cdot \boldsymbol{\sigma}_{N}}{M_{N}}, \boldsymbol{\sigma}_{N}+\frac{\mathbf{p}_{N} \cdot \boldsymbol{\sigma}_{N}}{M_{N}\left(M_{N}+p_{N}^{0}\right)} \mathbf{p}_{N}\right]
\end{aligned}
$$


where $W_{1}^{N\left(t_{N}\right)}$ and $W_{2}^{N\left(t_{N}\right)}$ are the spin-averaged nucleonic structure functions, while $G_{1}^{N\left(t_{N}\right)}$ and $G_{2}^{N\left(t_{N}\right)}$ are the spin-dependent nucleonic structure functions.

For elastic scattering from the nucleon, the structure functions are determined by the elastic Sachs form factors $G_{E}^{N\left(t_{N}\right)}$ and $G_{M}^{N\left(t_{N}\right)}$ :

$$
\begin{aligned}
W_{1}^{N\left(t_{N}\right)}\left(Q_{N}^{2}, \frac{q_{N} \cdot p_{N}}{M_{N}}\right)= & \tau\left[G_{M}^{N\left(t_{N}\right)}\left(Q_{N}^{2}\right)\right]^{2} \\
& \times 2 M_{N} \delta\left(2 q_{N} \cdot p_{N}-Q_{N}^{2}\right) \\
W_{2}^{N\left(t_{N}\right)}\left(Q_{N}^{2}, \frac{q_{N} \cdot p_{N}}{M_{N}}\right)= & \frac{\left[G_{E}^{N\left(t_{N}\right)}\left(Q_{N}^{2}\right)\right]^{2}+\tau\left[G_{M}^{N\left(t_{N}\right)}\left(Q_{N}^{2}\right)\right]^{2}}{1+\tau} \\
& \times 2 M_{N} \delta\left(2 q_{N} \cdot p_{N}-Q_{N}^{2}\right) \\
G_{1}^{N\left(t_{N}\right)}\left(Q_{N}^{2}, \frac{q_{N} \cdot p_{N}}{M_{N}}\right)= & -\frac{G_{M}^{N\left(t_{N}\right)}\left(Q_{N}^{2}\right)}{2} \frac{G_{E}^{N\left(t_{N}\right)}\left(Q_{N}^{2}\right)+\tau G_{M}^{N\left(t_{N}\right)}\left(Q_{N}^{2}\right)}{1+\tau} \\
& \times 2 M_{N} \delta\left(2 q_{N} \cdot p_{N}-Q_{N}^{2}\right) \\
G_{2}^{N\left(t_{N}\right)}\left(Q_{N}^{2}, \frac{q_{N} \cdot p_{N}}{M_{N}}\right)= & \frac{G_{M}^{N\left(t_{N}\right)}\left(Q_{N}^{2}\right)}{4} \frac{G_{M}^{N\left(t_{N}\right)}\left(Q_{N}^{2}\right)-\tau G_{E}^{N\left(t_{N}\right)}\left(Q_{N}^{2}\right)}{1+\tau} \\
& \times 2 M_{N} \delta\left(2 q_{N} \cdot p_{N}-Q_{N}^{2}\right)
\end{aligned}
$$

with

$$
\tau=\frac{Q_{N}^{2}}{4 M_{N}^{2}}
$$

\section{Spin-Dependent ${ }^{3} \mathrm{He}$ Spectral Function}

The spin-dependent nuclear spectral function is an operator in the nucleonic and nuclear spin space, whose matrix elements are defined by [8]

$$
\begin{aligned}
\left\langle s_{N} s_{A}^{\prime}\left|S\left(\mathbf{p}_{N}, E, t_{N}\right)\right| s_{N}^{\prime} s_{A}\right\rangle= & A \sum_{\substack{s_{A-1} f_{A-1}\\
}} \delta\left(E+E_{A}-e_{A-1}\left(f_{A-1}\right)\right) \\
& \times\left\langle\mathbf{P}_{A} s_{A}^{\prime} \mid \mathbf{p}_{N} s_{N}^{\prime} t_{N}\left(\mathbf{P}_{A}-\mathbf{p}_{N}\right) s_{A-1} f_{A-1}\right\rangle
\end{aligned}
$$




$$
\left\langle\mathbf{p}_{N} s_{N} t_{N}\left(\mathbf{P}_{A}-\mathbf{p}_{N}\right) s_{A-1} f_{A-1} \mid \mathbf{P}_{A} s_{A}\right\rangle
$$

where $E_{A}$ is the nuclear binding energy, $f_{A-1}$ are quantum numbers of excitation in the residual $A-1$ nucleus, and $e_{A-1}\left(f_{A-1}\right)$ is the excitation energy of this residual nucleus. Since the tensor structure of the nuclear current tensor in PWIA is carries by the nucleonic current tensor, the spectral function must be a Lorenz scalar. In the nuclear center-of-mass system it is a scalar with respect to rotation and parity transformation, thus it has the following general operator form

$$
\begin{aligned}
S\left(\mathbf{p}_{N}, E, t_{N}\right)= & \frac{1}{2}\left\{f_{0}\left(\left|\mathbf{p}_{N}\right|, E, t_{N}\right)+f_{1}\left(\left|\mathbf{p}_{N}\right|, E, t_{N}\right) \boldsymbol{\sigma}_{N} \cdot \boldsymbol{\sigma}_{A}\right. \\
& \left.+f_{2}\left(\left|\mathbf{p}_{N}\right|, E, t_{N}\right)\left[\left(\boldsymbol{\sigma}_{N} \cdot \hat{\mathbf{p}}_{N}\right)\left(\boldsymbol{\sigma}_{A} \cdot \hat{\mathbf{p}}_{N}\right)-\frac{1}{3} \boldsymbol{\sigma}_{N} \cdot \boldsymbol{\sigma}_{A}\right]\right\}
\end{aligned}
$$

where $f_{0}\left(\left|\mathbf{p}_{N}\right|, E, t_{N}\right)$ is the spin-averaged part of the spectral function while $f_{0}\left(\left|\mathbf{p}_{N}\right|, E, t_{N}\right)$ and $f_{0}\left(\left|\mathbf{p}_{N}\right|, E, t_{N}\right)$ are the spin-dependent ones.

The target nucleus with spin $\frac{1}{2}$ is characterized by the general polarization vector $\hat{\mathbf{n}}_{A}$ in its center-of-mass system

$$
\begin{array}{r}
\boldsymbol{\sigma}_{A} \cdot \hat{\mathbf{n}}_{A}\left|n_{A}\right\rangle=\left|n_{A}\right\rangle \\
\rho_{A}\left(\hat{\mathbf{n}}_{A}\right)=\frac{1}{2}\left(1+\boldsymbol{\sigma}_{A} \cdot \hat{\mathbf{n}}_{A}\right)
\end{array}
$$

where the operator $\rho_{A}$ is the corresponding density operator and $\left|n_{A}\right\rangle$ denotes the polarization state, which can be expanded in terms of spin states $\left|s_{A}\right\rangle$ with the $z$ axis being the quantization axis $\left|n_{A}\right\rangle=\sum_{s_{A}}\left|s_{A}\right\rangle\left\langle s_{A} \mid n_{A}\right\rangle$. Define a new four-vector

$$
\begin{aligned}
\mathcal{S}\left(\mathbf{p}_{N}, E, t_{N}, \hat{\mathbf{n}}_{A}\right)= & \operatorname{Tr}\left[s\left(\boldsymbol{\sigma}_{N}\right) S\left(\mathbf{p}_{N}, E, t_{N}\right) \rho_{A}\left(\hat{\mathbf{n}}_{A}\right)\right] \\
= & s\left(\hat{\mathbf{n}}_{A}\right)\left[f_{1}\left(\left|\mathbf{p}_{N}\right|, E, t_{N}\right)-\frac{1}{3} f_{2}\left(\left|\mathbf{p}_{N}\right|, E, t_{N}\right)\right]+ \\
& s\left(\hat{\mathbf{p}}_{N}\right)\left(\hat{\mathbf{n}}_{A} \cdot \hat{\mathbf{p}}_{N} f_{2}\left(\left|\mathbf{p}_{N}\right|, E, t_{N}\right)\right)
\end{aligned}
$$


where $\hat{\mathbf{n}}_{A}$ and $\hat{\mathbf{p}}_{N}$ are boosted by the nucleon momentum $\mathbf{p}_{N}$ to

$$
\begin{aligned}
& s\left(\hat{\mathbf{n}}_{A}\right)=\left[\frac{\mathbf{p}_{N} \cdot \hat{\mathbf{n}}_{A}}{M_{N}}, \hat{\mathbf{n}}_{A}+\frac{\mathbf{p}_{N} \cdot \hat{\mathbf{n}}_{A}}{M_{N}\left(M_{N}+p_{N}^{0}\right)} \mathbf{p}_{N}\right] \\
& s\left(\hat{\mathbf{p}}_{N}\right)=\left[\frac{\left|\mathbf{p}_{N}\right|}{M_{N}}, \hat{\mathbf{p}}_{N} \frac{p_{N}^{0}}{M_{N}}\right]
\end{aligned}
$$

With the nucleonic current tensor and the nuclear spectral function at hand, the nuclear structure functions in PWIA can be written as [8]

$$
\begin{aligned}
W_{1}^{A}= & \sum_{t_{N}} \int d^{3} p_{N} \frac{M_{N}}{p_{N}^{0}} \int d E\left\{W_{1}^{N\left(t_{N}\right)}+\frac{W_{2}^{N\left(t_{N}\right)}}{2 M_{N}^{2}}\left|\hat{\mathbf{q}} \times \mathbf{p}_{N}\right|^{2}\right\} f_{0}\left(\left|\mathbf{p}_{N}\right|, E, t_{N}\right) \\
W_{2}^{A}= & \sum_{t_{N}} \int d^{3} p_{N} \frac{M_{N}}{p_{N}^{0}} \int d E\left\{W_{1}^{N\left(t_{N}\right)} \frac{Q^{2}}{\mathbf{q}^{2}}\left[1-\frac{Q^{2}}{Q_{N}^{2}}\right]\right. \\
& \left.+\frac{W_{2}^{N\left(t_{N}\right)}}{M_{N}^{2}}\left[\frac{Q^{4}}{\mathbf{q}^{4}}\left(p_{N}^{0}+q_{N}^{0} \frac{q_{N} \cdot p_{N}}{Q_{N}^{2}}\right)^{2}+\frac{1}{2} \frac{Q^{2}}{\mathbf{q}^{2}}\left|\hat{\mathbf{q}} \times \mathbf{p}_{N}\right|^{2}\right]\right\} f_{0}\left(\left|\mathbf{p}_{N}\right|, E, t_{N}\right) \\
G_{1}^{A}= & \frac{M_{A}^{2}}{\mathbf{q}^{2}} \sum_{t_{N}} \int d^{3} p_{N} \frac{M_{N}}{p_{N}^{0}} \int d E\left[\frac{Q^{2}}{M_{A}} \frac{1}{n_{A}^{1}} g^{1 \alpha}+\frac{q^{0}}{M_{A}} \frac{1}{n_{A}^{3}}\left(q_{N}^{0} g^{3 \alpha}-|\mathbf{q}| g^{0 \alpha}\right)\right] \times \\
& {\left[\mathcal{S}_{\alpha}\left(\mathbf{p}_{N}, E, t_{N}, \hat{\mathbf{n}}_{A}\right) \frac{G_{1}^{N}}{M_{N}}+\left\{\left(q_{N} \cdot p_{N}\right) \mathcal{S}_{\alpha}\left(\mathbf{p}_{N}, E, t_{N}, \hat{\mathbf{n}}_{A}\right)-q_{N} \cdot \mathcal{S}\left(\mathbf{p}_{N}, E, t_{N}, \hat{\mathbf{n}}_{A}\right) p_{N \alpha}\right\} \frac{G_{2}^{N}}{M_{N}^{3}}\right] } \\
G_{2}^{A}= & -\frac{M_{A}^{2}}{\mathbf{q}^{2}} \sum_{t_{N}} \int d^{3} p_{N} \frac{M_{N}}{p_{N}^{0}} \int d E\left[q^{0} \frac{1}{n_{A}^{1}} g^{1 \alpha}+\frac{1}{n_{A}^{3}}\left(q_{N}^{0} g^{3 \alpha}-|\mathbf{q}| g^{0 \alpha}\right)\right] \times \\
& {\left[\mathcal{S}_{\alpha}\left(\mathbf{p}_{N}, E, t_{N}, \hat{\mathbf{n}}_{A}\right) \frac{G_{1}^{N}}{M_{N}}+\left\{\left(q_{N} \cdot p_{N}\right) \mathcal{S}_{\alpha}\left(\mathbf{p}_{N}, E, t_{N}, \hat{\mathbf{n}}_{A}\right)-q_{N} \cdot \mathcal{S}\left(\mathbf{p}_{N}, E, t_{N}, \hat{\mathbf{n}}_{A}\right) p_{N \alpha}\right\} \frac{G_{2}^{N}}{M_{N}^{3}}\right] }
\end{aligned}
$$

which can be numerically calculated.

\subsubsection{Non-Relativistic Full Faddeev Calculations}

The Faddeev decomposition of the three- and four-body problem has proven to be a tremendous computational tool in studies of light nuclei. In addition to being useful for studies of bound states and low-energy scattering, the Faddeev decomposition can 
be readily applied to higher-energy scattering problems. Using the Faddeev decomposition, the coordinate-space Schrödinger equation for three nucleons with two-nucleon interactions can be written as three Faddeev equations, with each equation involving only the interaction between one pair of the nucleons, thus achieving a significant simplification [35]. In momentum space the three Faddeev equations can be written as three integral equations.

First applications of the Faddeev formalism to unpolarized $p d$ and $p p n$ electrodisintegration of ${ }^{3} \mathrm{He}$ were done in $[47,48]$ with full inclusion of all final-state rescattering processes. This calculation was subsequently applied to electrodisintegration of polarized ${ }^{3} \mathrm{He}$ in [10] and later in [49] with the additional inclusion of meson-exchange currents.

The response functions can be written as [10]

$$
\begin{aligned}
R_{L} & =\sum_{m^{\prime} \tau^{\prime}} \int d f^{\prime} \delta\left(M+\omega-P_{0}^{\prime}\right)\left|N_{0}\right|^{2} \\
R_{T} & =\sum_{m^{\prime} \tau^{\prime}} \int d f^{\prime} \delta\left(M+\omega-P_{0}^{\prime}\right)\left(\left|N_{1}\right|^{2}+\left|N_{-1}\right|^{2}\right) \\
R_{T^{\prime}} & =\sum_{m^{\prime} \tau^{\prime}} \int d f^{\prime} \delta\left(M+\omega-P_{0}^{\prime}\right)\left(\left|N_{1}\right|^{2}-\left|N_{-1}\right|^{2}\right) \\
R_{T L^{\prime}} & =\sum_{m^{\prime} \tau^{\prime}} \int d f^{\prime} \delta\left(M+\omega-P_{0}^{\prime}\right) 2 \operatorname{Re}\left[-N_{0}\left(N_{1}+N_{-1}\right)^{*}\right]
\end{aligned}
$$

where the summation over all spin and isospin magnetic quantum numbers and momenta in the final state is indicated by $m^{\prime}, \tau^{\prime}$ and $d f^{\prime}$. The nuclear matrix elements $N_{0}$ and $N_{ \pm 1}$ are

$$
\begin{aligned}
N_{0} & =\left\langle\Psi_{f^{\prime} m^{\prime} \tau^{\prime}}^{(-)}|\rho(\vec{q})| \Psi_{3 \mathrm{He}}\right\rangle \\
N_{ \pm 1} & =\left\langle\Psi_{f^{\prime} m^{\prime} \tau^{\prime}}^{(-)}\left|j_{ \pm 1}(\vec{q})\right| \Psi_{3 \mathrm{He}}\right\rangle
\end{aligned}
$$

where $\left|\Psi_{3} \mathrm{He}\right\rangle$ is the ${ }^{3} \mathrm{He}$ ground state, and $\left|\Psi_{f^{\prime} m^{\prime} \tau^{\prime}}^{(-)}\right\rangle$is a $3 \mathrm{~N}$ scattering state with the asymptotic quantum numbers $f^{\prime} m^{\prime} \tau^{\prime} . \rho(\vec{q})$ is the electromagnetic hadronic charge 
density operator and $j_{ \pm 1}(\vec{q})$ are the spherical components of the electromagnetic hadronic current operator. In the nonrelativistic framework, the argument of the $\delta$-function is

$$
\begin{aligned}
M+\omega-P_{0}^{\prime} & =\epsilon_{3} \mathrm{He}+\omega-\frac{\vec{q}^{2}}{6 m_{N}}-E_{f^{\prime}} \\
& \equiv E-E_{f^{\prime}}
\end{aligned}
$$

where $\epsilon_{3} \mathrm{He}$ is the ${ }^{3} \mathrm{He}$ binding energy (negative), $m_{N}$ the nucleon mass, the final total momentum $\vec{P}^{\prime}=\vec{Q}$ and $E_{f^{\prime}}$ the internal $3 \mathrm{~N}$ energy related to the quantum numbers $f^{\prime}$.

Define

$$
\begin{aligned}
\mathcal{R}_{A B} & \equiv \sum_{m^{\prime} \tau^{\prime}} \int d f^{\prime} \delta\left(E-E_{f^{\prime}}\right)\left\langle\Psi_{f^{\prime} m^{\prime} \tau^{\prime}}^{(-)}|A| \Psi_{3 \mathrm{He}}\right\rangle\left\langle\Psi_{f^{\prime} m^{\prime} \tau^{\prime}}^{(-)}|B| \Psi_{3 \mathrm{He}}\right\rangle^{*} \\
& =\sum_{m^{\prime} \tau^{\prime}} \int d f^{\prime}\left\langle\Psi_{3 \mathrm{He}}\left|B^{\dagger}\right| \Psi_{f^{\prime} m^{\prime} \tau^{\prime}}^{(-)}\right\rangle \delta\left(E-E_{f^{\prime}}\right)\left\langle\Psi_{f^{\prime} m^{\prime} \tau^{\prime}}^{(-)}|A| \Psi_{3} \mathrm{He}\right\rangle \\
& =\sum_{m^{\prime} \tau^{\prime}} \int d f^{\prime}\left\langle\Psi_{3} \mathrm{He}\right. \\
& \left.\left|B^{\dagger} \delta(E-H)\right| \Psi_{f^{\prime} m^{\prime} \tau^{\prime}}^{(-)}\right\rangle\left\langle\Psi_{f^{\prime} m^{\prime} \tau^{\prime}}^{(-)}|A| \Psi_{3} \mathrm{He}\right\rangle \\
& =\left\langle\Psi_{3} \mathrm{He}\left|B^{\dagger} \delta(E-H) A\right| \Psi_{3} \mathrm{He}\right\rangle
\end{aligned}
$$

where $H$ is the $3 \mathrm{~N}$ Hamiltonian, then the four structure functions can be expressed as

$$
\begin{aligned}
R_{L} & =\mathcal{R}_{\rho \rho} \\
R_{T} & =\mathcal{R}_{j_{+1} j_{+1}}+\mathcal{R}_{j_{-1} j_{-1}} \\
R_{T^{\prime}} & =\mathcal{R}_{j_{+1} j_{+1}}-\mathcal{R}_{j_{-1} j_{-1}} \\
R_{T L^{\prime}} & =-2 \operatorname{Re}\left[\mathcal{R}_{j_{+1} \rho}+\mathcal{R}_{j_{-1} \rho}\right]
\end{aligned}
$$


Introducing scattering states

$$
\begin{aligned}
\left|\Psi_{A}^{( \pm)}\right\rangle & \equiv \frac{1}{E \mp i \epsilon-H} A\left|\Psi_{3} \mathrm{He}\right\rangle \\
\left|\Psi_{B}^{(+)}\right\rangle & \equiv \frac{1}{E+i \epsilon-H} B\left|\Psi_{3 \mathrm{He}}\right\rangle
\end{aligned}
$$

then

$$
\begin{aligned}
\mathcal{R}_{A B} & =\frac{1}{2 \pi i}\left\langle\Psi_{3 \mathrm{He}}\left|B^{\dagger} \frac{1}{E-i \epsilon-H} A\right| \Psi_{3} \mathrm{He}\right\rangle-\frac{1}{2 \pi i}\left\langle\Psi_{3 \mathrm{He}}\left|B^{\dagger} \frac{1}{E+i \epsilon-H} A\right| \Psi_{3} \mathrm{He}\right\rangle \\
& \equiv \frac{1}{2 \pi i}\left\langle\Psi_{3 \mathrm{He}}\left|B^{\dagger}\right| \Psi_{A}^{(-)}\right\rangle-\frac{1}{2 \pi i}\left\langle\Psi_{3} \mathrm{He}\left|B^{\dagger}\right| \Psi_{A}^{(+)}\right\rangle \\
& =\frac{1}{2 \pi i}\left(\left\langle\Psi_{3} \mathrm{He}\left|A^{\dagger}\right| \Psi_{B}^{(+)}\right\rangle^{*}-\left\langle\Psi_{3} \mathrm{He}\left|B^{\dagger}\right| \Psi_{A}^{(+)}\right\rangle\right)
\end{aligned}
$$

The states $\left|\Psi_{A, B}^{(+)}\right\rangle$, defined in Eqs. (1.71) and (1.71) contain all the complexity of the interaction among the three nucleons and are evaluated as in [3,50] using the Faddeev scheme. The result is

$$
\Psi_{C}^{(+)}=G_{0}(1+P) U_{C}
$$

with

$$
U_{C}=\left(1+t G_{0}\right) C^{(1)}\left|\Psi_{3 \mathrm{He}}\right\rangle+t G_{0} P U_{C}
$$

where $t$ is the NN t-matrix, which accounts for the rescattering between the final state nucleons. In this way FSI effects are naturally incorporated in the Faddeev formalism. $G_{0}$ is the free $3 \mathrm{~N}$ propagator and $P$ is the sum of a cyclic and anticyclic permutation of 3 objects. $C$ is either $A$ or $B$ (for instance $\rho$ or $j_{ \pm}$) and can be decomposed as

$$
C=\sum_{i=1}^{3} C^{(i)}
$$


and each current operator $C^{(i)}$ can be further decomposed into two parts, the single particle current operator and the two-body current operator [49]

$$
C^{(1)}=C_{\text {sing }}^{(1)}+C_{e x c h}^{(23)}
$$

where the two-body currents follow from the Riska prescription, which via the continuity equation relates $N N$ forces and exchange currents in a model independent manner. In this way the meson-exchange currents effects are also naturally included.

To solve the Faddeev equation (1.74) numerically, a set of standard basis in momentum space is introduced [51]

$$
|p q \alpha \mathcal{J} M\rangle=\left|p q(l s) j\left(\lambda \frac{1}{2}\right) J \mathcal{J} M\left(t \frac{1}{2}\right) T M_{T}\right\rangle
$$

where $\mathrm{p}$ and $\mathrm{q}$ are magnitudes of Jacobi momenta and the set of discrete quantum numbers $\alpha$ comprises angular momenta, spins and isospins for a three-nucleon system.

The ${ }^{3}$ He state polarized in the direction $\theta^{*}, \phi^{*}$ is

$$
\left|\Psi_{3} \mathrm{He}^{m}\right\rangle_{\theta^{*} \phi^{*}}=\sum_{m^{\prime}} \mid \Psi_{3} \mathrm{He}^{\left.m^{\prime}\right\rangle} D_{m^{\prime}, m}^{(1 / 2)}\left(-\phi^{*},-\theta^{*}, 0\right)
$$

where $\left|\Psi_{3} \mathrm{He}^{m}\right\rangle$ is quantized with respect to the z-direction and the Wigner D-function occurs as

$$
D_{m^{\prime}, m}^{(1 / 2)}\left(-\phi^{*},-\theta^{*}, 0\right)=e^{-i m^{\prime} \phi^{*}}\left(\begin{array}{cc}
\cos \frac{\theta^{*}}{2} & -\sin \frac{\theta^{*}}{2} \\
\sin \frac{\theta^{*}}{2} & \cos \frac{\theta^{*}}{2}
\end{array}\right)
$$

With the above formalism, the response functions can finally be written as [10,52]:

$$
\begin{aligned}
& R_{L}=-\frac{3}{\pi} \operatorname{Im}\left[\sum \frac{1}{E+i \epsilon-\frac{p^{2}}{m}-\frac{3}{4 m} q^{2}}\left\langle p q \alpha \mathcal{J} \frac{1}{2}\left|(1+P) \rho^{(1)}\right| \Psi_{3} \mathrm{He}_{2}\right\rangle\left\langle p q \alpha \mathcal{J} \frac{1}{2} \mid U_{\rho} \frac{1}{2}\right\rangle\right] \quad(1.80) \\
& R_{T}=-\frac{3}{\pi} \operatorname{Im}\left[\sum \frac { 1 } { E + i \epsilon - \frac { p ^ { 2 } } { m } - \frac { 3 } { 4 m } q ^ { 2 } } \left(\left\langle p q \alpha \mathcal{J} \frac{1}{2}\left|(1+P) j_{1}^{(1)}\right| \Psi_{3} \mathrm{He}-\frac{1}{2}\right\rangle^{*}\left\langle p q \alpha \mathcal{J} \frac{1}{2} \mid U_{j_{1}}-\frac{1}{2}\right\rangle\right.\right.
\end{aligned}
$$




$$
\begin{aligned}
& \left.\left.+\left\langle p q \alpha \mathcal{J} \frac{3}{2}\left|(1+P) j_{1}^{(1)}\right| \Psi_{3} \mathrm{He}_{2}\right\rangle^{*}\left\langle p q \alpha \mathcal{J} \frac{3}{2} \mid U_{j_{1}} \frac{1}{2}\right\rangle\right)\right] \\
R_{T L^{\prime}}= & \pm \frac{3}{\pi} \operatorname{Im}\left[\sum \frac { 1 } { E + i \epsilon - \frac { p ^ { 2 } } { m } - \frac { 3 } { 4 m } q ^ { 2 } } \left(\left\langle p q \alpha \mathcal{J} \frac{1}{2}\left|(1+P) j_{1}^{(1)}\right| \Psi_{3} \mathrm{He}-\frac{1}{2}\right\rangle^{*}\left\langle p q \alpha \mathcal{J} \frac{1}{2} \mid U_{\rho} \frac{1}{2}\right\rangle\right.\right. \\
& \left.\left.-\left\langle p q \alpha \mathcal{J} \frac{1}{2}\left|(1+P) \rho^{(1)}\right| \Psi_{3} \mathrm{He} \frac{1}{2}\right\rangle\left\langle p q \alpha \mathcal{J} \frac{1}{2} \mid U_{j_{1}}-\frac{1}{2}\right\rangle^{*}\right)\right] \sin \theta^{*} \cos \phi^{*} \\
R_{T^{\prime}}= & \pm \frac{3}{\pi} \operatorname{Im}\left[\sum \frac{1}{E+i \epsilon-\frac{p^{2}}{m}-\frac{3}{4 m} q^{2}}\right. \\
& \left(\left\langle p q \alpha \mathcal{J} \frac{1}{2}\left|(1+P) j_{1}^{(1)}\right| \Psi_{3} \mathrm{He}-\frac{1}{2}\right\rangle^{*}\left\langle p q \alpha \mathcal{J} \frac{1}{2} \mid U_{j_{1}}-\frac{1}{2}\right\rangle\right. \\
& \left.\left.-\left\langle p q \alpha \mathcal{J} \frac{3}{2}\left|(1+P) j_{1}^{(1)}\right| \Psi_{3} \mathrm{He}_{\frac{1}{2}}\right\rangle^{*}\left\langle p q \alpha \mathcal{J} \frac{3}{2} \mid U_{j_{1}} \frac{1}{2}\right\rangle\right)\right] \cos \theta^{*}
\end{aligned}
$$

where the sums in $\mathcal{Y}$ include the summation over the magnetic quantum number $M$ of the total $3 \mathrm{~N}$ angular momentum $\mathcal{J}$. The response functions thus obtained can be calculated numerically with the computing power of supercomputers. 


\section{Chapter 2}

\section{The Experimental Apparatus}

\subsection{Overview}

The Thomas Jefferson National Accelerator Facility (Jefferson Lab), located in Newport News, Virginia, is one of the most powerful microscopes in the world for studying the quark structure of matter and the underlying strong interaction. Designed to be the world's premier medium energy electron scattering laboratory, the facility's construction was started in 1987 and the first physics experiments were performed in 1994.

The Lab consists of a state-of-the-art continuous-wave electron accelerator, three complementary experimental halls, named Hall A, Hall B and Hall C, that utilize the electron beam to explore different aspects of nuclear and particle physics, a free electron laser (FEL) facility and an applied research center. Figure 2-1 is an aerial view of the Lab.

The accelerator (Figure 2-2) is capable of delivering high quality continuous electron beam of up to $6 \mathrm{GeV}$ to the three halls simultaneously, and is planned to be upgraded to $12 \mathrm{GeV}$ in the near future. The beam is generated in the following manner. Electrons are initially accelerated to $45 \mathrm{MeV}$ at the injector site, from where they are delivered to a racetrack-shaped accelerator, which consists of two $400 \mathrm{MeV}$ 


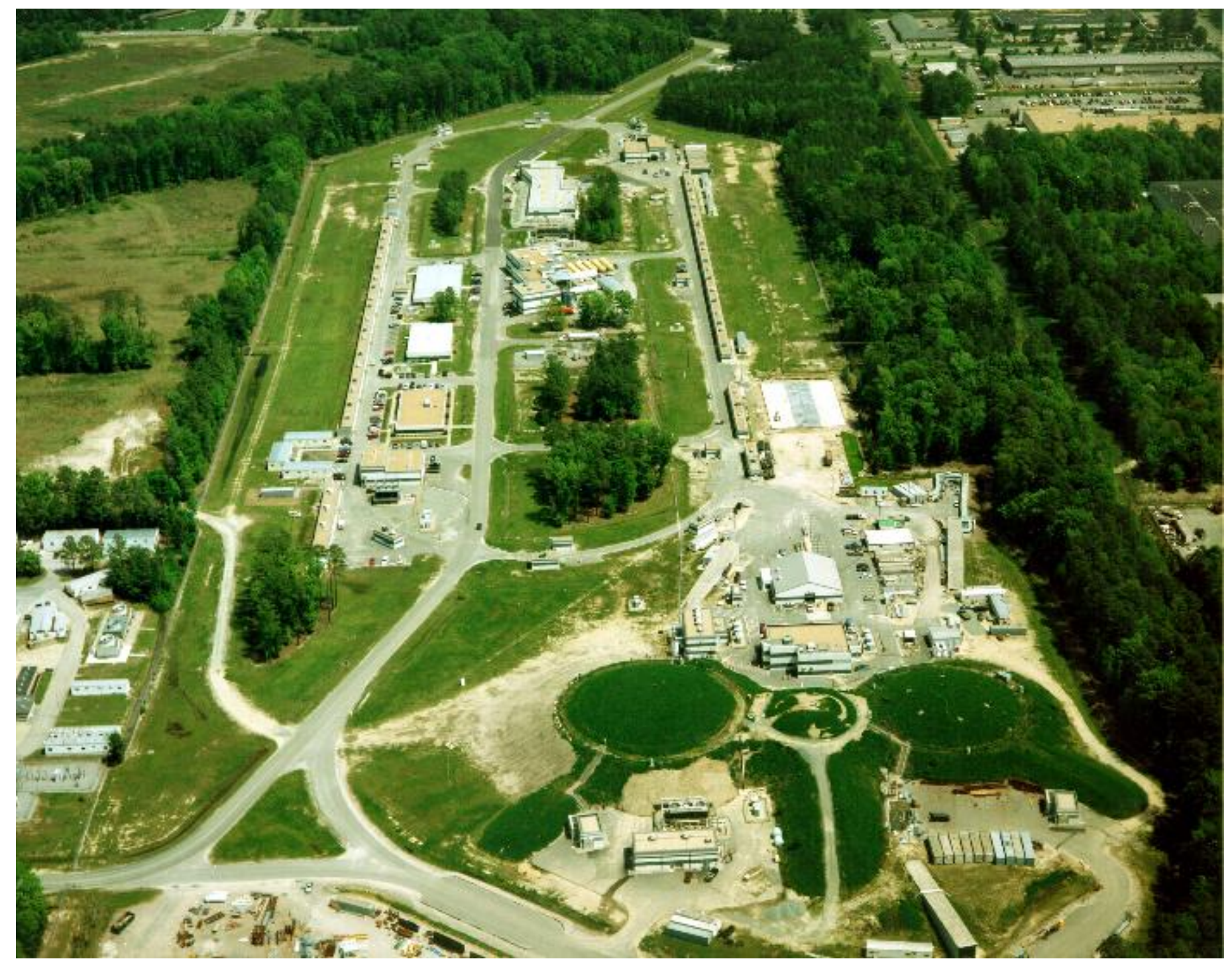

Figure 2-1: An aerial view of Jefferson Lab accelerator site and the three experimental halls. 


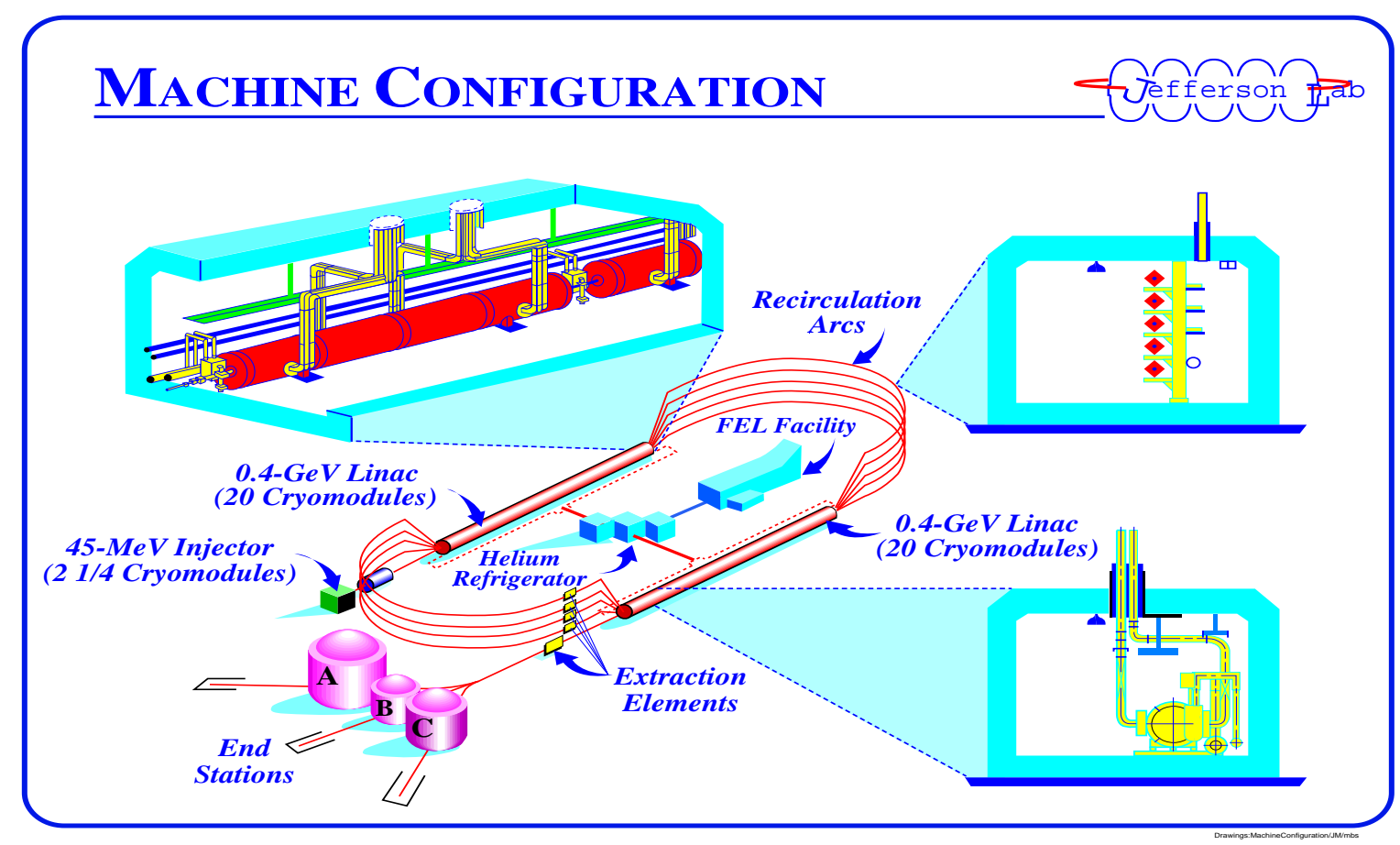

Figure 2-2: Schematics of JLab accelerator configuration.

linacs and two recirculation arcs. The electrons pass through the first linac and pick up $400 \mathrm{MeV}$. Then they travel through a $180^{\circ}$ turn in the first recirculation arc and acquire another $400 \mathrm{MeV}$ while they pass down the second linac. Now the electrons can either be delivered to one of the three experimental halls, or enter the second recirculation arc and start another pass. The $400 \mathrm{MeV}$ linac acceleration is a nominal amount, in reality electrons can acquire up to $1.2 \mathrm{GeV}$ with each pass. A total of up to five passes are allowed which amounts to a maximum beam energy of about 6 $\mathrm{GeV}$.

Experiment E95-001 was conducted in Hall A in Spring 1999. There were two goals to this experiment. The first was to measure the transverse asymmetry $A_{T^{\prime}}$ on top of the quasielastic peak of ${ }^{3} \mathrm{He}\left(\vec{e}, e^{\prime}\right)$ and then to extract the neutron magnetic form factor $G_{M}^{n}$. The second goal was to measure the spin-dependent asymmetry in the threshold region of ${ }^{3} \mathrm{He}\left(\vec{e}, e^{\prime}\right)$. Two beam energies, $778 \mathrm{MeV}$ at one pass and 
$1727 \mathrm{MeV}$ at two passes, were used during the experiment and the average beam current was $10 \mu \mathrm{A}$, which was due to the limitation of the polarized ${ }^{3} \mathrm{He}$ target. Figure 2-3 shows the Hall A configuration during the experiment. The beam was longitudinally polarized at the injector and the polarization was determined with a Møller polarimeter. The beam energy was measured using an Arc Energy Device while the beam current and position were monitored continuously using Beam Current Monitors (BCMs) and Beam Position Monitors (BPMs). The polarized electron beam was incident on a $40 \mathrm{~cm}$ polarized ${ }^{3} \mathrm{He}$ target cell, based on the principle of spinexchange optical pumping, where the reaction took place. The scattered electrons passed through two High Resolution Spectrometers, HRSe and HRSh, focused by three quadrupoles and deflected by a dipole to the focal plane. Both spectrometers were configured to detect electrons in single-arm mode using nearly identical detector packages consisting of two dual-plane vertical drift chambers (VDCs) for tracking, two planes of segmented plastic scintillators for trigger formation, and a $\mathrm{CO}_{2}$ gas Čerenkov detector and lead-glass total-absorption shower counter for pion rejection. The HRSe was set for quasi-elastic kinematics while the HRSh covered both the elastic peak and the threshold region.

\subsection{Polarized Electron Beam}

\subsubsection{Beam source}

The polarized electron source at Jefferson Lab is similar to the one used at SLAC [53]. The electron beam is generated by illuminating a strained GaAs cathode, placed in a ultra-high vacuum, with high intensity circularly polarized laser light.

The strained GaAs cathode is created by growing GaAs on a substrate of $\mathrm{GaAs}_{0.72} \mathrm{P}_{0.28}$ as shown in the left panel of Figure 2-4. The shorter lattice spacing of $\mathrm{GaAs}_{0.72} \mathrm{P}_{0.28}$, which is $5.5968 \AA$, causes the natural spacing of GaAs, which is $5.6533 \AA$, to shrink a little bit, thus creating strain in the GaAs crystal. This strain splits the sublevels of 


\section{Jlab E95-001 Floor Configuration}

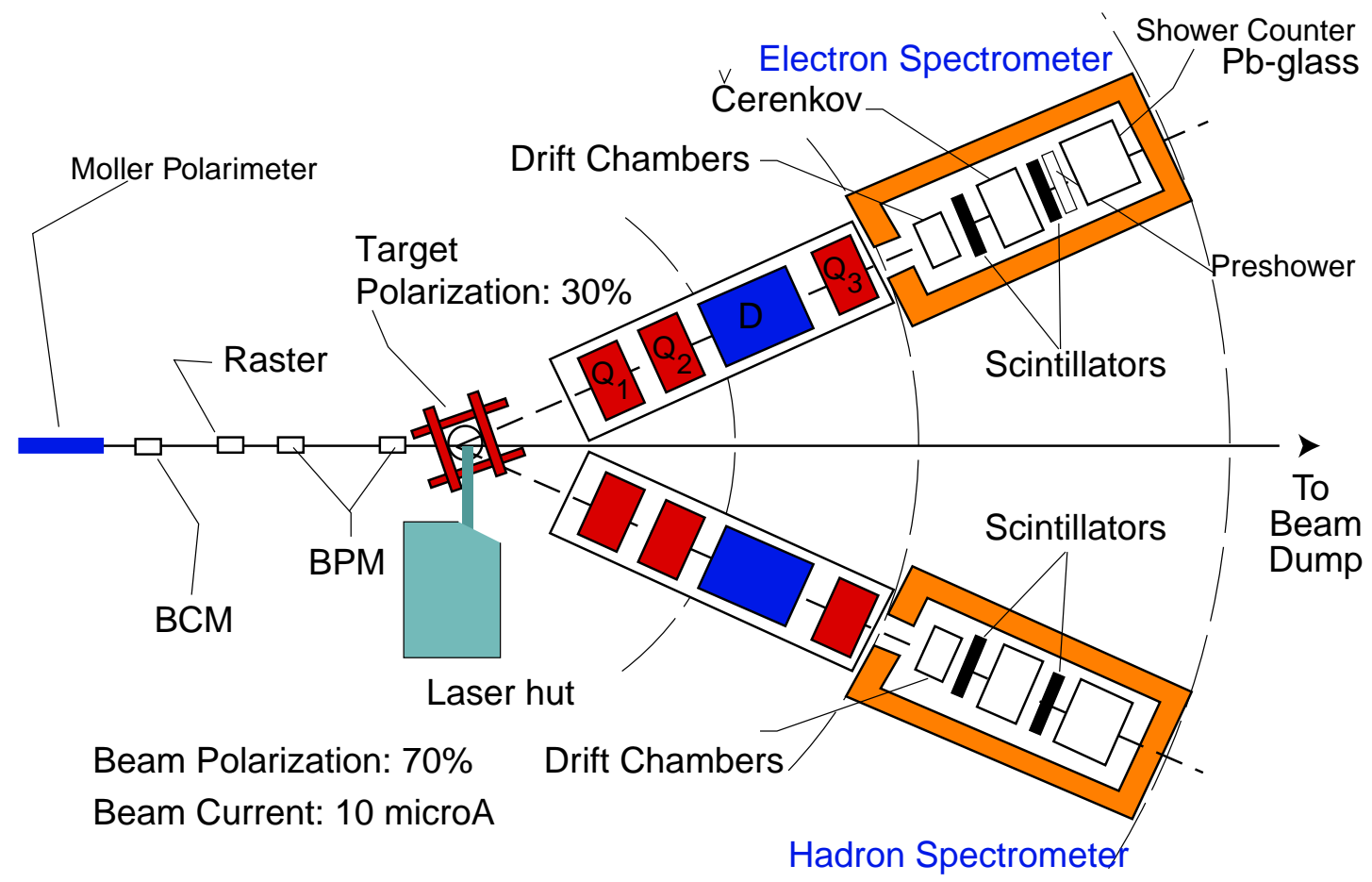

Figure 2-3: Hall A Configuration for E95-001.

the $\mathrm{P}_{3 / 2}$ electrons in the valence band of GaAs, making the $\mathrm{m}=3 / 2$ sublevel higher than $\mathrm{m}=1 / 2$ sublevel (see the right panel of Figure 2-4). By tuning a circularly polarized laser to the proper frequency, electrons from the $\mathrm{P}_{3 / 2} \mathrm{~m}=3 / 2$ sublevel can be excited to the $\mathrm{S}_{3 / 2} \mathrm{~m}=1 / 2$ sublevel in the conduction band. A very thin film of Cs-F is deposited over the GaAs layer to lower the energy of the conduction band below the vacuum energy, thus allowing the excited electrons to escape from the crystal into the vacuum. The cathode is maintained at a negative voltage of $60-120 \mathrm{kV}$ which gives the electrons the initial acceleration. Because the strain creates a sufficiently wide gap between the $\mathrm{P}_{3 / 2}$ sublevels, electrons in the $\mathrm{m}=1 / 2$ state will not be excited by the laser. Thus theoretically the electrons leaving the surface of the cathodes can be $100 \%$ polarized. In practice, beam polarization over $70 \%$ has been achieved.

The incident laser is circularly polarized using two Pockels cells, one acting as a 
half waveplate and the other as a quarter waveplate. The helicity of the laser, thus the helicity of the electron beam, can be flipped using the half waveplate Pockels cell. The flip rate was $1 \mathrm{~Hz}$ in the beginning of the experiment and was later changed to $30 \mathrm{~Hz}$.

\subsubsection{M $\varnothing$ ller Polarimeter}

The beam polarization measurement is based on the process of Møller scattering, which is simply elastic scattering of incoming electrons on target electrons, which in this case are atomic electrons in a target. Consider the scattering of a longitudinally polarized electron incident on a polarized target electron. The cross section in the center-of-mass system is

$$
\frac{d \sigma}{d \Omega}=\left(\frac{d \sigma}{d \Omega}\right)_{0}\left(1+P_{B} P_{T} A\left(\theta_{C M}\right)\right)
$$

with

$$
A\left(\theta_{C M}\right)=\frac{\left(7+\cos ^{2} \theta_{C M}\right) \sin ^{2} \theta_{C M}}{\left(3+\cos ^{2} \theta_{C M}\right)^{2}}
$$

where $\left(\frac{d \sigma}{d \Omega}\right)_{0}$ is the unpolarized cross section, $P_{B}$ is the beam polarization, $P_{T}$ is the target polarization projected onto the beam direction, and $\theta_{C M}$ is the center-of-mass
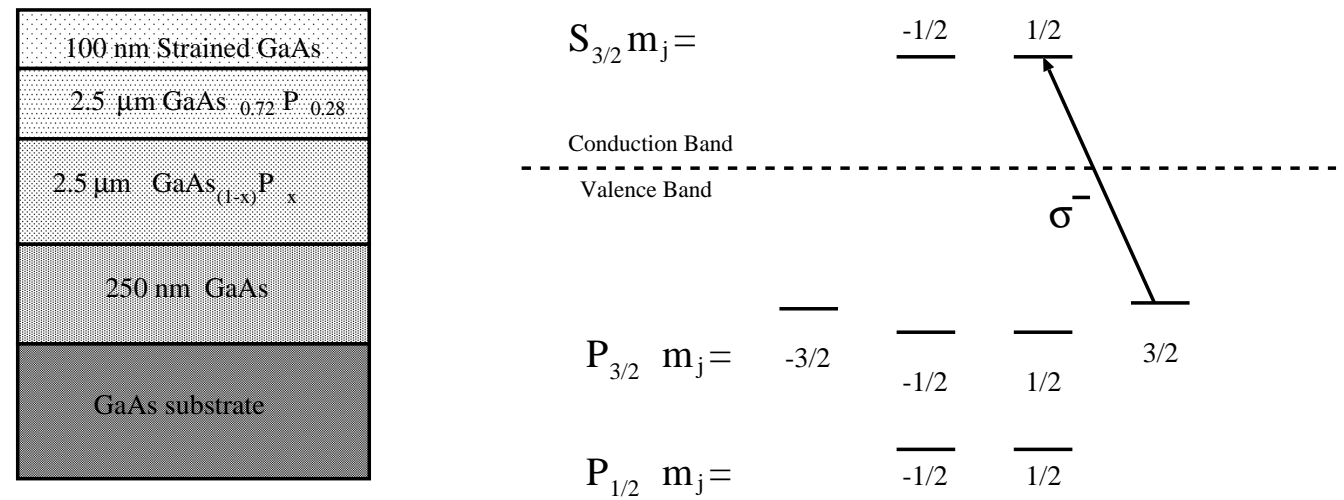

Figure 2-4: The different layers of strained GaAs cathode and the level diagram of the conduction and valence bands of GaAs. 
scattering angle. When $\theta_{C M}=90^{\circ}, A\left(\theta_{C M}\right)$ achieves a maximum of $7 / 9$.

The measured asymmetry

$$
A_{m}=\frac{\sigma^{\uparrow \uparrow}-\sigma^{\uparrow \downarrow}}{\sigma^{\uparrow \uparrow}-\sigma^{\uparrow \downarrow}}=P_{B} P_{T} A\left(\theta_{C M}\right)
$$

is proportional to the beam and target polarization and can be used to determine the beam polarization if the target polarization is known.

The Møller polarimeter in Hall A is shown in Figure 2-5. It uses a $12 \mu \mathrm{m}$ thick supermendur foil (Fe plated onto a $\mathrm{Cu}$ foil) as the target. This is a soft ferromagnetic material and is driven to saturation by placing it in a $300 \mathrm{G}$ magnetic field. Due to boundary conditions, 2 electrons out of 26 in Fe will be polarized along the foil plane, thus the foil polarization $P_{\text {foil }}$ is about $7.69 \%$. Ideally the foil should be placed along the beam line to maximize the asymmetry, which is not practical because it is very thin. During the actual measurement the foil was tilted at an angle $\theta_{t g t}$ from $20^{\circ}$ to $160^{\circ}$ with respect to the beam line, resulting in an effective target polarization of $P_{\text {eff }}=P_{\text {foil }} \times \cos \theta_{t g t}$.

Scattered electrons are detected by two Møller spectrometers in coincidence to improve the signal-to-noise ratio. Each spectrometer is comprised of three quadrupoles and one dipole, which are followed by one scintillator and one lead-glass shower counter. The angular acceptance of the spectrometer ranges from $76^{\circ}$ to $104^{\circ}$ in the center-of-mass system. Taking into account the acceptance effect, the average value of $A\left(\theta_{C M}\right)$ is 0.759 as determined by a Monte-Carlo simulation.

Six Møller measurements were taken in our experiment and the results are shown in Table 2.1 together with the statistical and systematic errors.

\subsubsection{Beam Current Monitors}

The beam current was measured using two Beam Current Monitors (BCMs) placed in the beamline about $24.5 \mathrm{~m}$ upstream of the target (Figure 2-6). The BCM is simply 


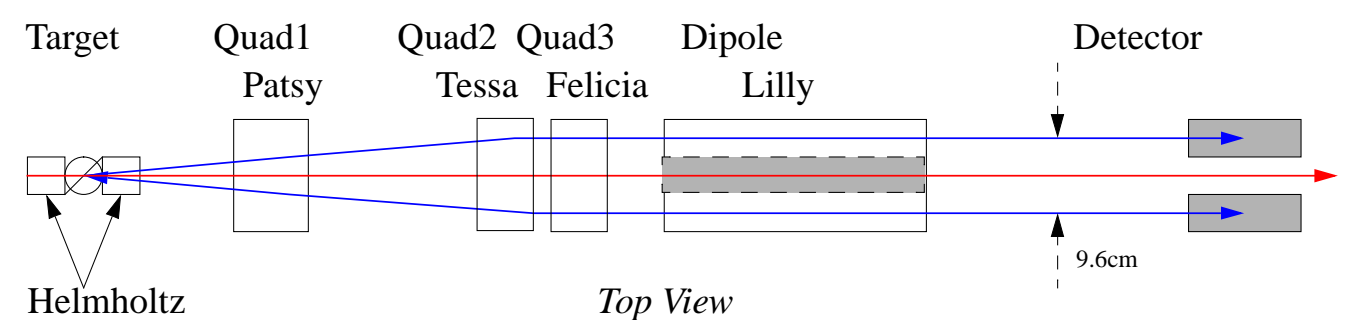

coils Moller Polarimeter

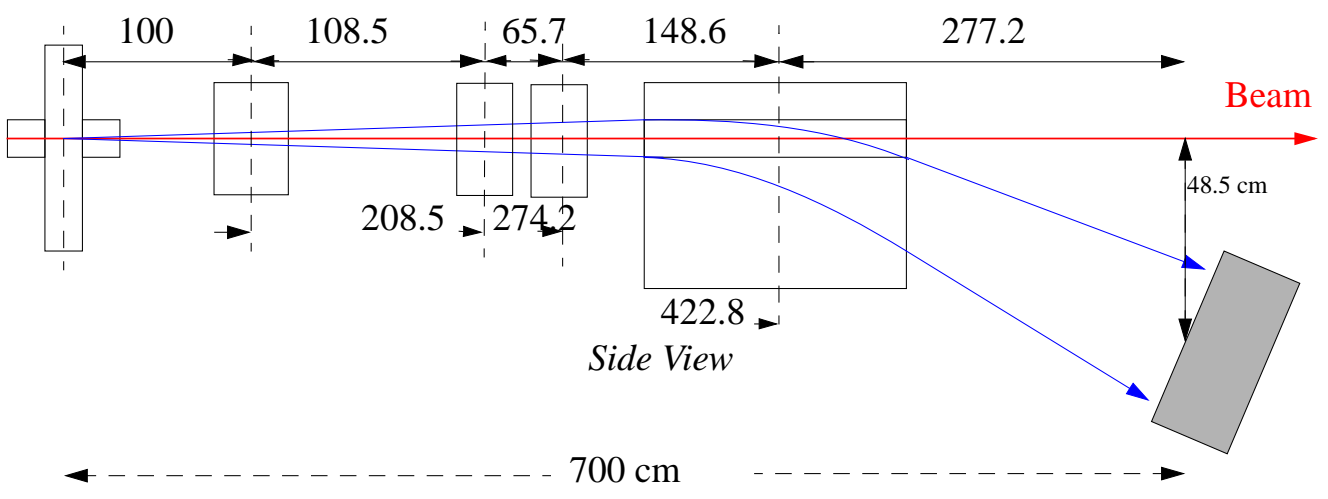

Figure 2-5: Schematics of Hall A Møller polarimeter.

a cylindrical resonant cavity tuned to the frequency of the electron beam, which is $1497 \mathrm{MHz}$ at JLab. Inside each cavity there are two coaxial loop antennas. The outer one has a radius coupled to one of the resonant modes of the cavity, and is used to couple the beam signal out of the cavity. The inner one can be used to periodically test the response of the cavity by sending a $1497 \mathrm{MHz}$ calibration signal through it and detecting the output signal from the outer antenna. When the electron beam passes through the cavity, it excites the resonant transverse electromagnetic mode $\mathrm{TM}_{010}$ at $1497 \mathrm{MHz}$. The outer antenna would then detect this magnetic field and output a signal proportional to the beam current. 


\begin{tabular}{|c|c|c|c|}
\hline \hline Date & $P_{\text {beam }}(\%)$ & $\delta_{\text {stat }}(\%)$ & $\delta_{\text {sys }}(\%)$ \\
\hline Jan. 22 & 70.0 & 2.00 & 3.5 \\
Jan. 26 & 72.5 & 0.50 & 3.5 \\
Jan. 31 & 74.2 & 0.30 & 3.5 \\
Feb. 04 & 67.7 & 0.30 & 3.5 \\
Feb. 08 & 69.5 & 0.20 & 3.5 \\
Feb. 12 & 69.4 & 0.15 & 3.5 \\
Feb. 19 & 71.3 & 0.15 & 3.5 \\
Feb. 28 & 72.0 & 0.15 & 3.5 \\
\hline \hline
\end{tabular}

Table 2.1: Beam polarization results from Møller measurements.

Since the BCM output signal has a high frequency of $1497 \mathrm{MHz}$ and thus has high attenuation rate, it is first converted to a $1 \mathrm{MHz}$ signal using a down-converter installed close to each cavity. This $1 \mathrm{MHz}$ signal is then recorded in two ways. The first method records the signal every 4, 10 and 50 seconds, and sends all signals to a VME crate. The second method converts the voltage signal into a frequency signal using a Voltage-to-Frequency (VtoF) converter, which is then recorded by a scaler.

The BCMs have excellent linearity over a wide range of beam currents and thus can serve as accurate relative current monitors. However, they require an absolute calibration which is provided by an Unser monitor [54] sandwiched between them. The Unser uses a Direct Current Transformer which is composed of two identical toroidal cores driven in opposite ways by an external source. A common sensing wire is wound around both cores and in the absence of any beam current passing through the center of the toroids there is no signal from the sensing wire. A DC-current passing through the cores would produce a flux in-balance, and thus a signal in the sensing wire. The Unser is calibrated by passing a reference current, generated by a high precision current source, along a wire that is placed through the device along the beam line to simulate the beam current. The Unser itself cannot be used to continuously monitor the beam current because its output signal drifts significantly on a time scale of several minutes. Thus, it is only used to calibrate the BCMs. Since 


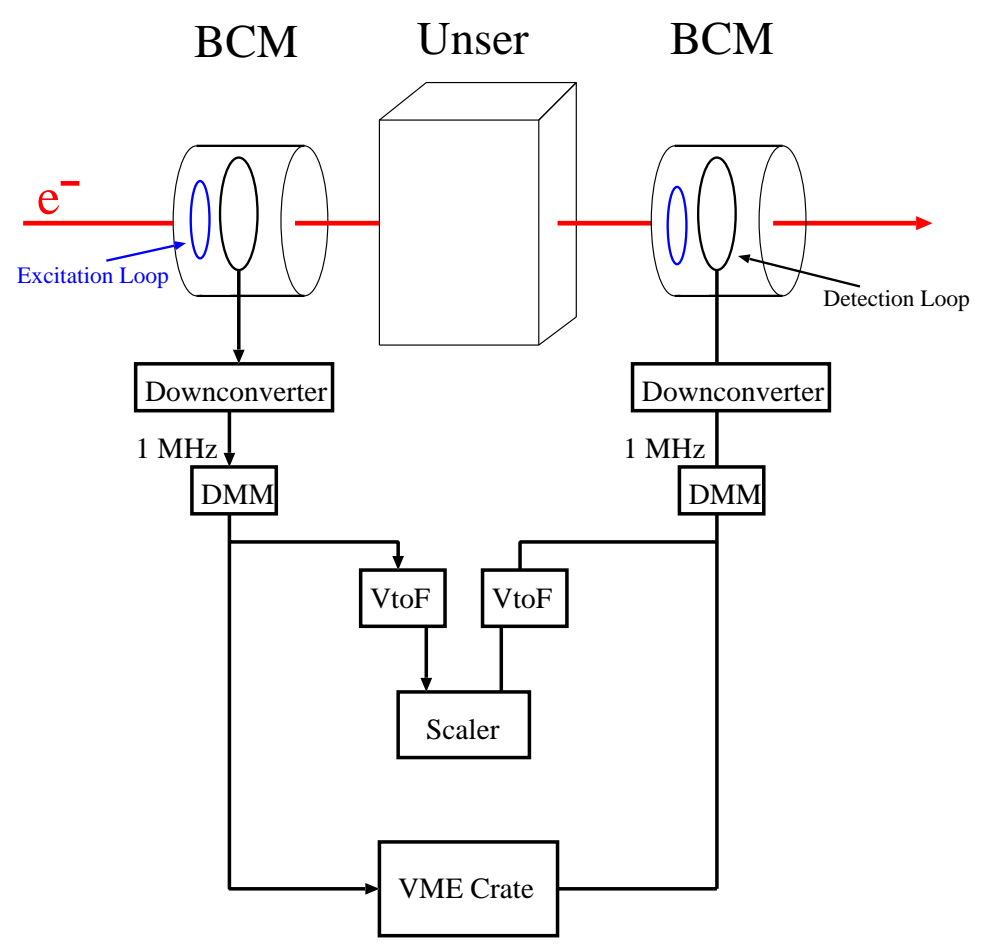

Figure 2-6: Schematics of Hall A Beam Current Monitor.

there is a constant uncertainty of about $250 \mathrm{nA}$ in the Unser measurement, the BCM calibration is better performed at a relatively high current to reduce the relative error. The calibration constant thus obtained can be used to convert the scaler counts from BCMs to the beam charge.

\subsubsection{Beam Position Monitors}

The beam position was monitored using two Beam Position Monitors (BPMs) located along the beamline and about 1 meter and 7 meters upstream of the target, respectively. The BPM is a cavity with four antennae surrounding the beam line in a diamond configuration (Figure 2-7). The signal picked up by each antenna from the electron beam is inversely proportional to the vertical distance between the beam and the antenna. The beam position can thus be accurately determined using the four signals. At a current of $10 \mu \mathrm{A}$, the beam position can be determined to about 


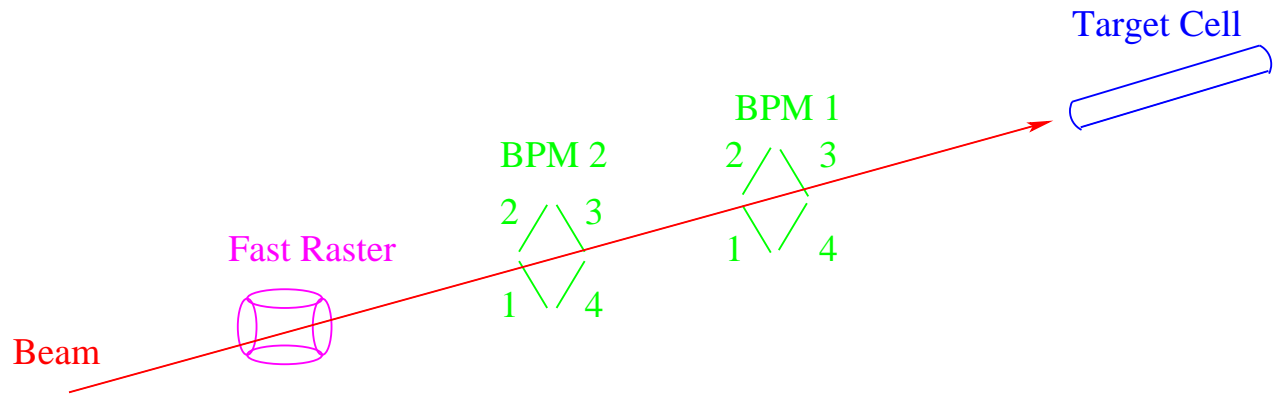

Figure 2-7: Schematics of Hall A Beam Position Monitors.

To reduce the effect of beam heating on the target cell entrance and exit windows, a fast raster was used in the experiment to slightly move the beam around the beamline. The size of the beam spot on the target entrance window was typically $3 \mathrm{~mm} \times 4$ $\mathrm{mm}$, and the beam center was kept to be within $0.5 \mathrm{~mm}$ from a nominal target center, which was selected to minimize the background during the experiment.

\subsubsection{Beam Energy}

In our experiment, three methods were used to determine the beam energy: measuring the electron trajectory in a magnetic field (Arc measurement), elastic electron-proton scattering (eP measurement) and elastic electron- ${ }^{3} \mathrm{He}$ scattering.

The Arc measurement is based on the principle that in a uniform magnetic field an electron moves in a circular pattern, the radius of which depends on the strength of the magnetic field and the electron energy. The Hall A Arc setup is shown in Figure 2-8. Before entering Hall A, the beam passes through a bend. The beam energy is determined by the following relation:

$$
E=c \frac{\int B_{\perp} d l}{\theta_{\|}}
$$

where $B_{\perp}$ is the magnetic field perpendicular to the beam path plane, $\int B_{\perp} d l$ is the 


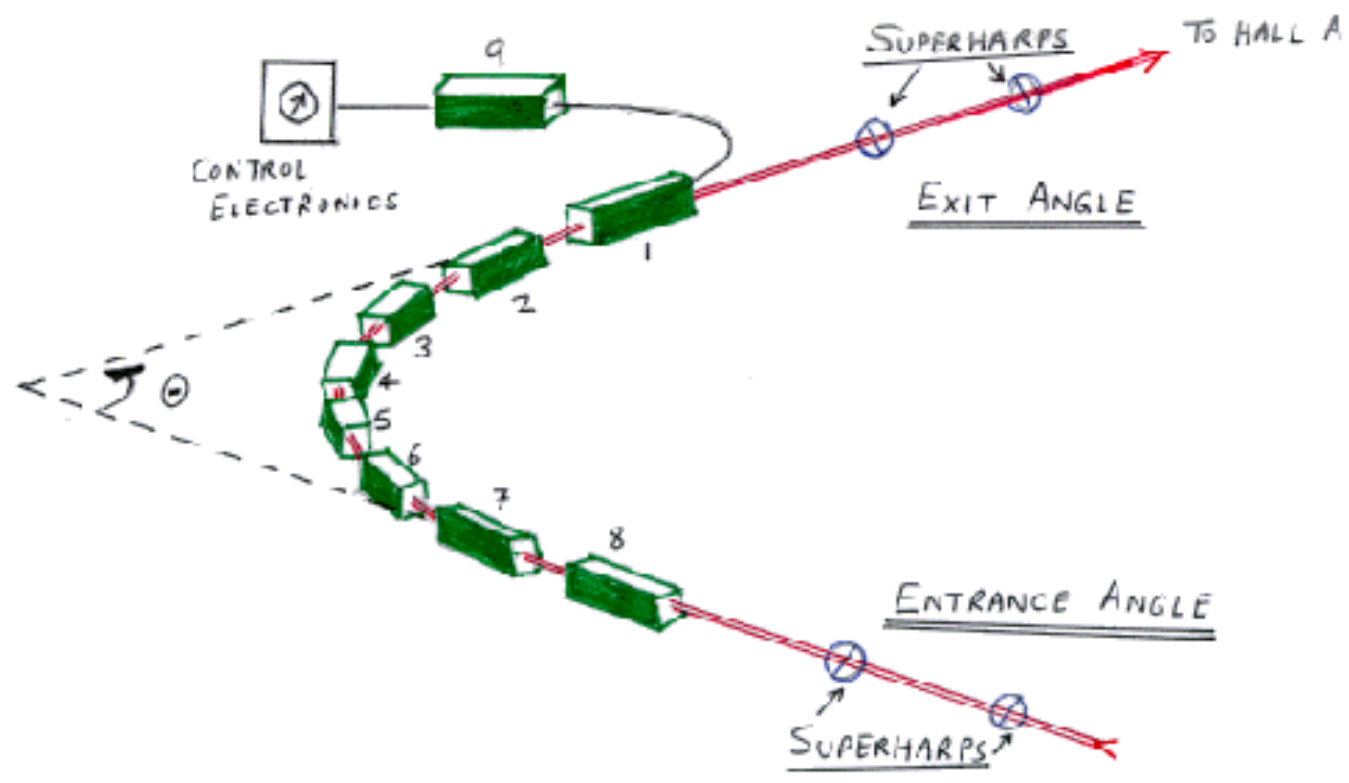

Figure 2-8: Hall A Arc Measurement.

integration of $B_{\perp}$ along the beam path, and $\theta_{\|}$is the angle by which the beam is deflected. $\theta_{\|}$is simply the difference between the entrance angle, which is determined by measuring two beam positions before the entrance to the bend, and the exit angle, which is determined by measuring two beam positions after the exit from the bend. The beam positions are measured using two sets of superharps. A superharp is a set of three wires that are spaced evenly apart. During the Arc measurement, the superharp is moved across the beam path. When the beam strikes a wire, a current is generated in that wire and the beam's position is determined. With the precise knowledge of $B_{\perp}$ and $\theta_{\|}$, the beam energy can be determined to a precision of $2 \times 10^{-4}$.

In eP elastic scattering, the electron energy can be determined from the knowledge of the angles of the scattered electron and the recoiling proton as follows:

$$
E=M_{p}\left(\cot \frac{\theta_{e}}{2} \cdot \cot \theta_{p}-1\right)
$$

where $\mathrm{M}_{p}$ is the proton mass, $\theta_{e}$ is the angle of the scattered electron and $\theta_{p}$ the angle of the recoiling proton. 


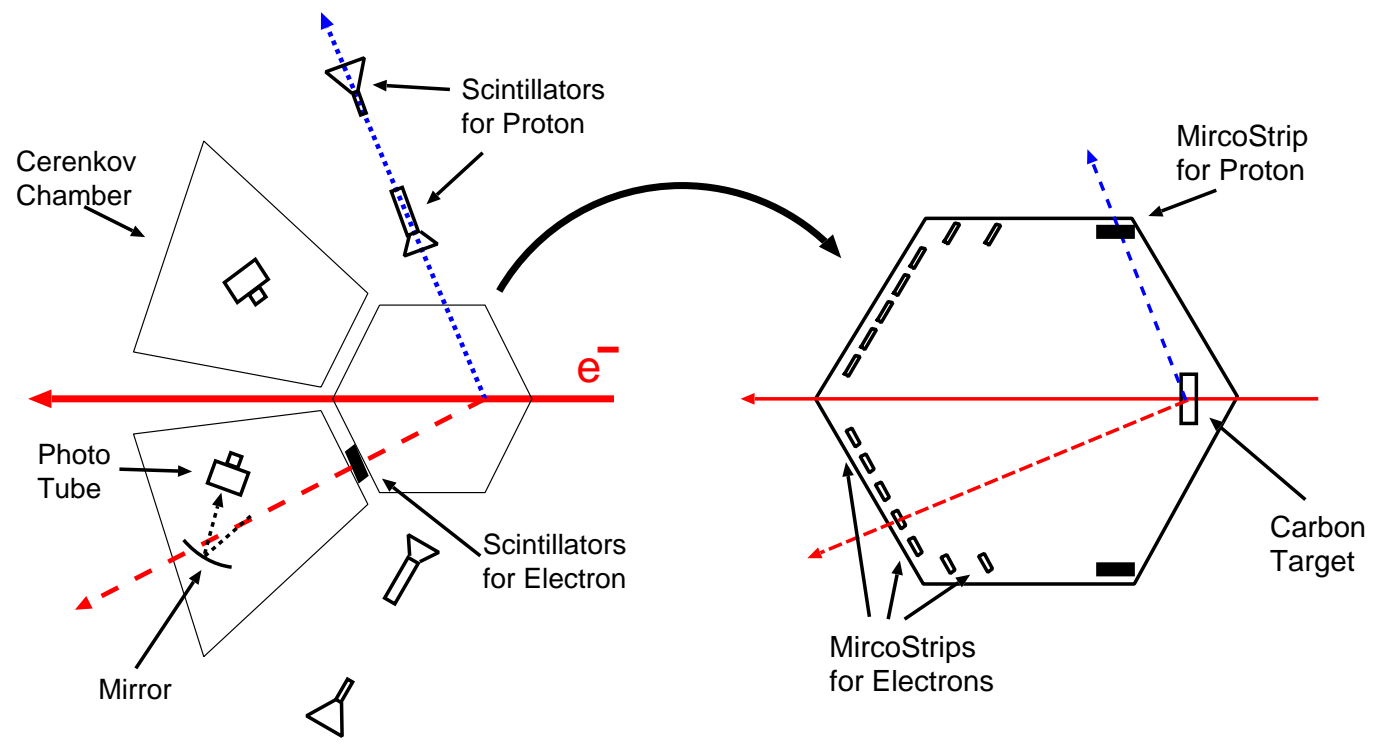

Figure 2-9: Hall A eP Measurement.

The eP measuring device in Hall A consists of a Čerenkov chamber, a few scintillators, a few silicon microstrips, and a $\mathrm{CH}_{2}$ target, as shown in Figure 2-9. Incident electrons get scattered by protons in the $\mathrm{CH}_{2}$ target. The scattered electrons first pass through a microstrip, then a scintillator and finally a Cerenkov chamber. The Cerenkov light is detected by a phototube. In a similar manner, the recoiling protons pass through a microstrip and then a set of scintillators. The coincidence signal between the electron and proton scintillators defines an event while the positions of the fired microstrips allow for a precise determination of both electron and proton angles. The eP measurement has an accuracy of $1 \times 10^{-4}$.

In the e- ${ }^{3} \mathrm{He}$ elastic scattering method, the beam energy is determined with knowledge of the energy and scattering angle of the scattered electron from data:

$$
E=\frac{E^{\prime}}{1-\frac{2 E^{\prime} \sin ^{2} \frac{\theta}{2}}{M_{3_{H e}}}}
$$

where $E^{\prime}$ and $\theta$ are the energy and scattering angle of the scattered electron, measured by the HRSh, and $\mathrm{M}_{3} \mathrm{He}$ is the mass of the ${ }^{3} \mathrm{He}$ nucleus. 


\subsection{Polarized ${ }^{3} \mathrm{He}$ Target}

Polarizing ${ }^{3} \mathrm{He}$ atoms has a long history dating back to 1960 s and has stimulated great interest and extensive efforts of decades of nuclear physicists ever since. The early applications of polarized ${ }^{3} \mathrm{He}$ targets include the development of ${ }^{3} \mathrm{He}$ magnetometers, which can be used to measure the earth and the interplanetary magnetic fields, and the study of quantum properties of polarized ${ }^{3} \mathrm{He}$ fluids at ultralow temperature.

With the advent of advanced laser technology, it has become feasible to develop polarized ${ }^{3} \mathrm{He}$ targets for electron scattering experiments. The basic technique is called optical pumping, which is a method of transferring angular momentum from polarized photons to the atoms, thereby polarizing the latter.

\subsubsection{Optical Pumping and Spin Exchange}

Traditionally optical pumping of an atom involves the transition of the ground state of the atom to the first excited state. However this is impractical for ${ }^{3} \mathrm{He}$ because it is difficult to produce laser beams with a high energy of $21 \mathrm{eV}(59 \mathrm{~nm})$, the excitation energy of the first excited state for ${ }^{3} \mathrm{He}$. Instead, two indirect methods have been developed to optically pump ${ }^{3} \mathrm{He}$ atoms.

The first one is called metastability-exchange optical pumping and was developed in the early 1960s [55]. The ${ }^{3} \mathrm{He}$ metastable $2{ }^{3} \mathrm{~S}_{1}$ atoms are produced using a weak radio-frequency $(\mathrm{RF})$ discharge on a glass cell filled with a few torr of pure ${ }^{3} \mathrm{He}$ gas. These $2^{3} \mathrm{~S}_{1}$ atoms are then pumped to $2^{3} \mathrm{P}_{0}$ states with $1083.4 \mathrm{~nm}$ circularly polarized photons. The sample is placed in a weak uniform magnetic field which defines the spin direction and thus only certain magnetic sublevels of $2^{3} \mathrm{~S}_{1}$ states will be excited to $2^{3} \mathrm{P}_{0}$ states, which then decay back to all sublevels of $2^{3} \mathrm{~S}_{1}$. The net effect is that the metastable atoms become polarized. A hyperfine interaction would then mix the electronic polarization into the nuclear polarization for these metastable atoms. The polarization of these atoms is subsequently transferred to the ground state through 
metastability-exchange collisions in which only the excitation of the electronic cloud is exchanged. Now if the ground state of a ${ }^{3} \mathrm{He}$ atom is polarized, the nucleus must be polarized since the atom is in a $\mathrm{J}=0$ state. This process can be expressed schematically as:

$$
{ }^{3} \mathrm{He}+{ }^{3} \overrightarrow{\mathrm{He}}^{*} \longrightarrow{ }^{3} \overrightarrow{\mathrm{He}}+{ }^{3} \mathrm{He}^{*}
$$

The ${ }^{3}$ He polarization can reach as high as $40 \%$ using this technique, but unfortunately it only works for low pressure ${ }^{3} \mathrm{He}$ gas target, because at high pressure the lifetime of the metastable atoms is drastically reduced and it is also difficult to maintain a uniform discharge under high pressure conditions.

The second method, which was employed in this experiment, involves the optical pumping of alkali metals and the spin exchange collisions between the polarized alkali atoms and ${ }^{3} \mathrm{He}$ nuclei. A review of optical pumping of alkali metals can be found in [56] and more recently in [57].

\section{Optical Pumping}

The alkali metal used in this experiment is rubidium, which has a single outer shell electron $\left(5 \mathrm{~S}_{1 / 2}\right)$. When placed in a magnetic field, the Hamiltonian for the system of this electron and the Rb nucleus is:

$$
H=A \mathbf{I} \cdot \mathbf{S}+g \mu_{B} S_{z} B_{z}-\frac{\mu_{I}}{I} I_{z} B_{z}
$$

where the first term shows the vector coupling of the electron spin $\mathbf{S}$ to the nuclear spin I, with a coupling strength of A. The last two terms show the coupling of the electron spin and nuclear spin to the external magnetic field, which we assume to be along z-axis, $\mathrm{B}_{z}$. The coupling strength depends on the $\mathrm{g}$ value for electron and the Bohr magneton $\mu_{B}$ for the electron spin, and the nuclear magnetic moment $\mu_{I}$ and the nuclear spin quantum number I for the nuclear spin, which is $5 / 2$ for ${ }^{85} \mathrm{Rb}(76.6 \%$ natural abundance) and $3 / 2$ for ${ }^{87} \mathrm{Rb}$ (23.4\% natural abundance).

Without the magnetic field $\left(\mathrm{B}_{z}=0\right)$, the electron eigenstates can be characterized 


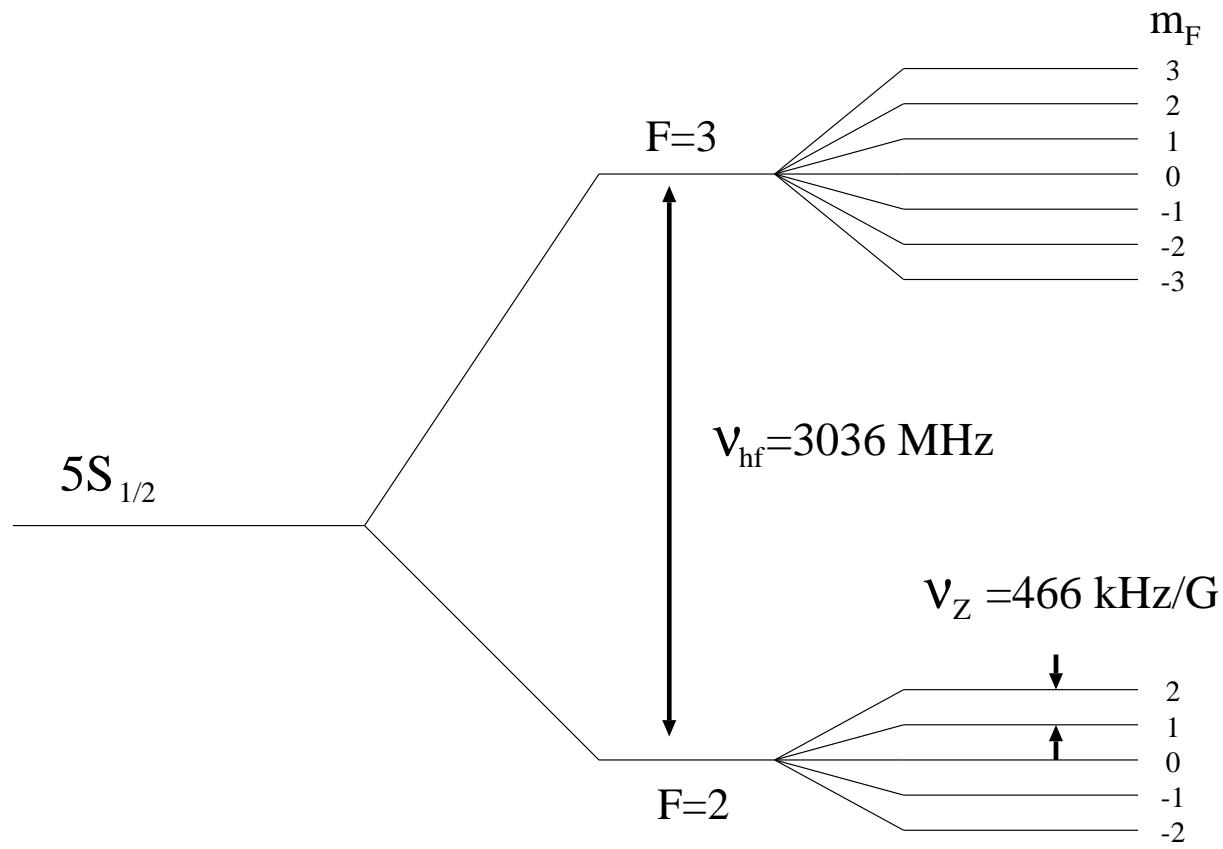

Figure 2-10: The energy level diagram for ${ }^{85} \mathrm{Rb}$ in an external magnetic field.

by the total spin quantum number $\mathrm{F}$, which equals 3 or 2 for ${ }^{85} \mathrm{Rb}$, and 2 or 1 for ${ }^{87} \mathrm{Rb}$. With the presence of a magnetic field, the energy level will be further divided due to the last two terms of Equation 2.8, and is shown in Figure 2-10 for the case of ${ }^{85} \mathrm{Rb}$.

During the optical pumping, a circularly polarized laser light with a wavelength of $795 \mathrm{~nm}$, which corresponds to the $\mathrm{D} 1$ transition $\left(5 \mathrm{~S}_{1 / 2} \longrightarrow 5 \mathrm{P}_{1 / 2}\right)$ of rubidium atoms, is applied to a sample of rubidium vapor placed in a uniform magnetic field. If the photon helicity is in the same direction as the magnetic field, then all electrons in the $5 \mathrm{~S}_{1 / 2}$ sublevels, except the $\mathrm{m}_{F}=3$ sublevel, will be excited to the $5 \mathrm{P}_{1 / 2}$ sublevels. The excited electrons will decay back to any of the sublevels in the ground state and thus in time all but the $\mathrm{m}_{F}=3$ sublevel will be depopulated and the atoms are polarized.

A simplified form of the optical pumping process is shown in Figure 2-11. The ${ }^{2} \mathrm{~S}_{1 / 2}$ electrons from the $\mathrm{m}_{j}=-1 / 2$ sublevel will be excited to the ${ }^{2} \mathrm{P}_{1 / 2} \quad \mathrm{~m}_{j}=1 / 2$ sublevel, from where they will decay to both the $\mathrm{m}_{j}=-1 / 2$ and the $\mathrm{m}_{j}=1 / 2$ sublevels 
in the ground state. After a while the $\mathrm{m}_{j}=-1 / 2$ ground state will be depopulated.

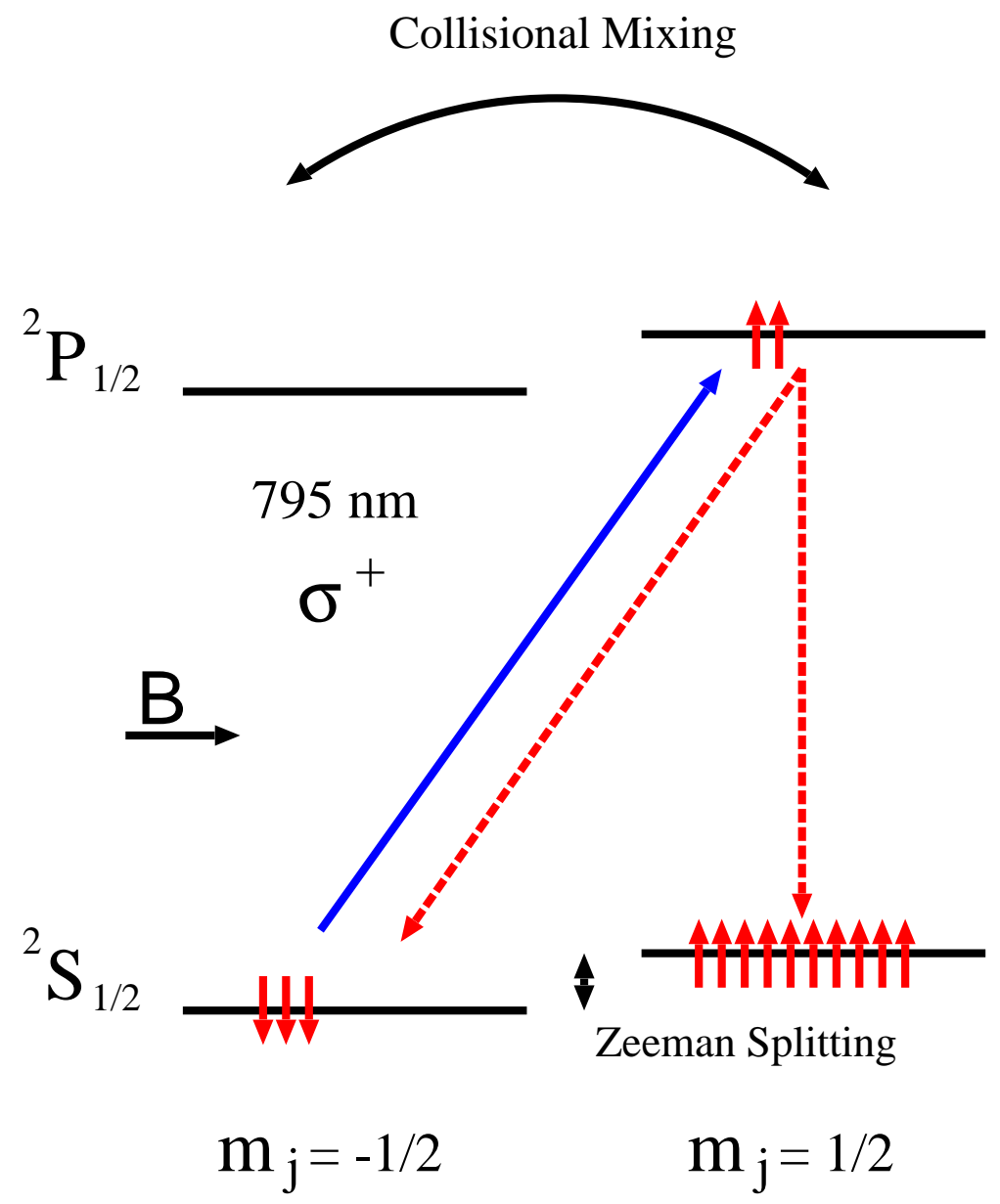

Figure 2-11: A simplified optical pumping process for rubidium.

A practical issue involves the photons emitted by excited electrons when they decay back to the ground state. These photons are randomly polarized and thus can excite the electrons in the $\mathrm{m}_{F}=3$ ground state, thereby reducing the polarization. A buffer gas $\mathrm{N}_{2}$ is introduced to reduce this depolarization effect through a process called quenching, whereby the collisions between rubidium atoms and $\mathrm{N}_{2}$ allow the electrons to decay without emitting photons, through the mechanical excitation of $\mathrm{N}_{2}$. The density of $\mathrm{N}_{2}$ gas is chosen to be a few orders of magnitude less than the density of ${ }^{3} \mathrm{He}$ gas, while a few orders of magnitude more than the density of rubidium atoms. Under this condition only about $5 \%$ of excited electrons would decay by emitting 
photons.

The polarization of the rubidium vapor $P_{R b}$ is determined by the optical pumping rate $(\mathrm{R})$ and the spin destruction rate $\left(\Gamma_{S D}\right)$ :

$$
P_{R b}=\frac{R}{R+\Gamma_{S D}}
$$

The spin destruction rate is typically much smaller than the pumping rate and thus the rubidium vapor is nearly $100 \%$ polarized in regions with abundant laser light.

\section{Spin Exchange}

When a rubidium atom collides with a ${ }^{3} \mathrm{He}$ nucleus, it will transfer part of its polarization to the latter via a weak hyperfine interaction between the ${ }^{3} \mathrm{He}$ nucleus and the outer electron of the rubidium atom. The ${ }^{3} \mathrm{He}$ polarization is given by:

$$
P^{{ }^{H}}{ }=\frac{\gamma_{S E}}{\gamma_{S E}+\Gamma}\left\langle P_{R b}\right\rangle
$$

where $\left\langle P_{R b}\right\rangle$ is the volume-averaged rubidium polarization, $\gamma_{S E}$ is the spin exchange rate, which is proportional to rubidium density, and $\Gamma$ is the ${ }^{3} \mathrm{He}$ nuclear spin relaxation rate in the absence of rubidium vapor and electron beam. For the target cell used in our experiment, $\gamma_{S E}$ was about $1 /(10 \mathrm{hrs})$, and $\Gamma$ was measured to be around $1 /(40 \mathrm{hrs})$. However the average rubidium polarization was not measured during the experiment and had to be extracted from the measured ${ }^{3} \mathrm{He}$ polarization.

\subsubsection{The JLab Hall A Polarized ${ }^{3} \mathrm{He}$ Target}

The JLab Hall A polarized ${ }^{3} \mathrm{He}$ target was built based on the design of a similar target used at SLAC [58]. As is shown in Fig. 2-12, there are four main systems to this target: an optical pumping system, a target system, a Nuclear Magnetic Resonance (NMR) system and an Electron Paramagnetic Resonance (EPR) system. The NMR system will be discussed in the next section. The EPR measurement was not used for our analysis work, so we will not discuss it here. The interested reader is encouraged to 
refer to [59] for details.

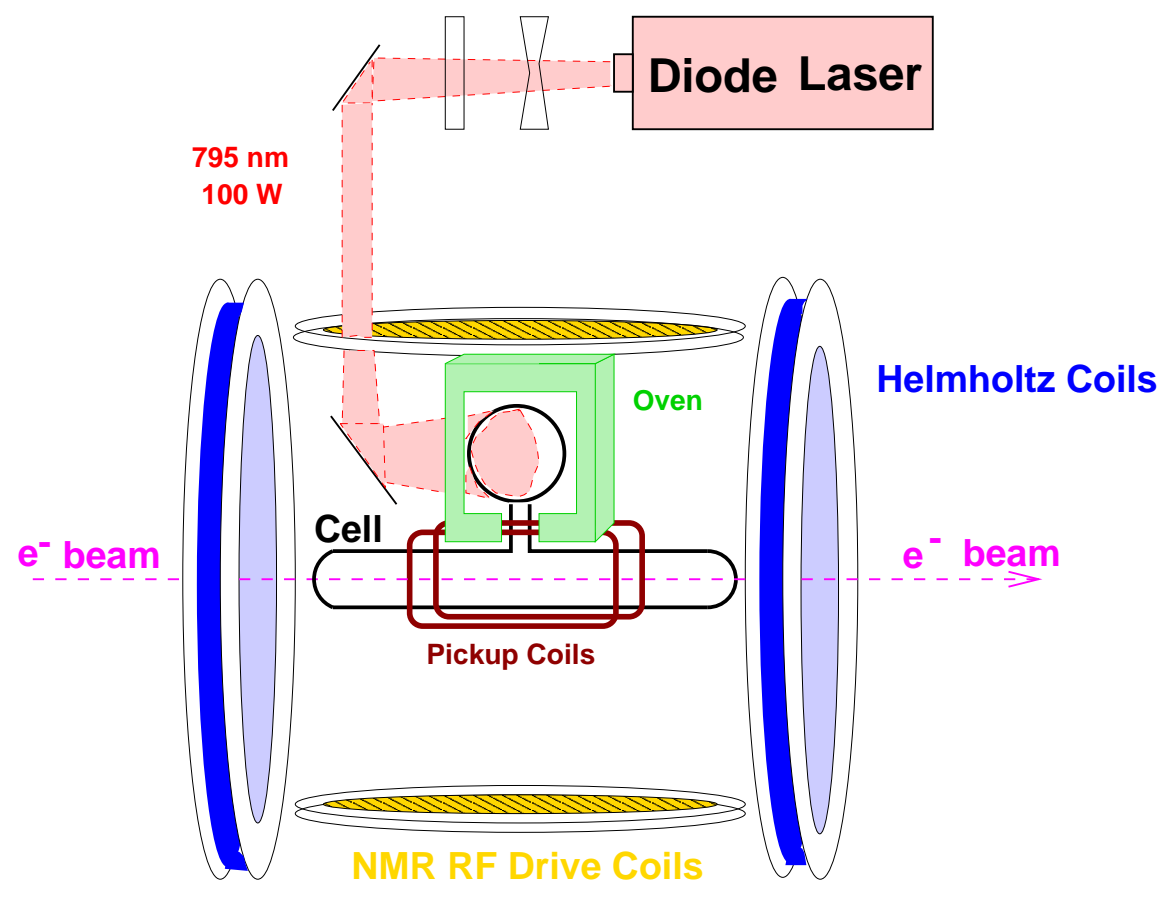

Figure 2-12: Schematics of Hall A polarized ${ }^{3} \mathrm{He}$ target.

\section{Optical Pumping System}

The optical pumping system is comprised of two sets of Helmholtz coils used to create a holding field in any direction in the scattering plane and the laser system used for optical pumping.

The two sets of Helmholtz coils are used to create a uniform field of about 20 Gauss around the target. They are perpendicular to each other and are turned $19^{\circ}$ off the beam line so as not to block the scattered particles. Both the magnitude and the direction of the holding field can be adjusted by varying the currents running in these two sets of coils.

The laser system consists of four diode lasers, a set of polarizing optics to circularly polarize the laser light and a set of mirrors and lenses to direct the light towards the target. Each diode laser can produce light with a central wavelength of $795 \mathrm{~nm}$ and a FWHM smaller than $2.5 \mathrm{~nm}$ and can achieve a maximum power of 30 Watts. The 
laser light is circularly polarized using a few polarizing cubes and quarter waveplates and the polarized light is directed by a few mirrors and lens onto the target, pointing $62.5^{\circ}$ to the right of the incident electron beam. The helicity of the lasers can be reversed remotely using a set of half waveplates. The whole system was shielded in a concrete hut near the target in Hall A for radiation protection.

\section{Target System}

The main ingredient of the target system is a ${ }^{3} \mathrm{He}$ target cell (Figure 2-13) where the optical pumping and spin exchanges processes actually take place.

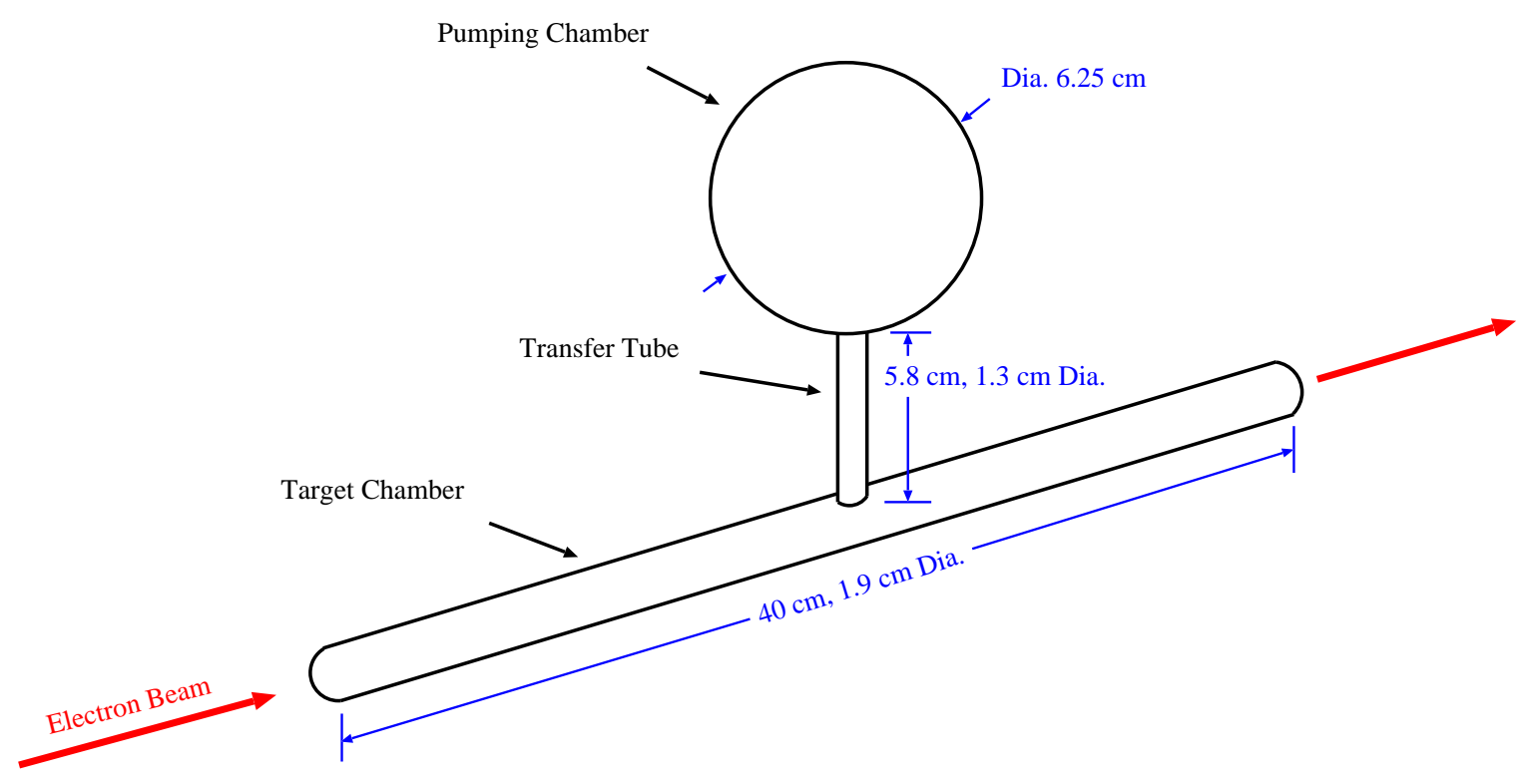

Figure 2-13: A typical ${ }^{3} \mathrm{He}$ target cell.

The target cell is made of aluminum-silicate glass (Corning 1720 or GE 180) and is a one-piece cell with three parts: a pumping chamber, a target chamber and a transfer tube. The pumping chamber is spherical and was placed within a plastic oven to control the rubidium vapor density during the experiment. The target chamber is a $40 \mathrm{~cm}$ tube which was placed along the electron beam during the experiment. The transfer tube simply connects the two chambers. During the construction of the target cell, first rubidium was chased into the pumping chamber and $\mathrm{N}_{2}$ gas was filled into the cell to about 40 torr. The target chamber was then cooled to about 10 
$\mathrm{K}$ to keep the pressure in the cell below 1 atm and ${ }^{3} \mathrm{He}$ gas was filled to a density such that the ${ }^{3} \mathrm{He}$ pressure would be $9-10$ atms in room temperature. The initial $\mathrm{N}_{2}$ pressure of about 40 torr was chosen so that the final $\mathrm{N}_{2}$ pressure was about $60-70$ torr in room temperature. For a detailed account of the cell-construction process, please refer to [59].

The target cell was mounted to a target ladder (Figure 2-14) which also has spaces for a reference cell and a $\mathrm{BeO}$ target. The reference cell can be filled with $\mathrm{N}_{2}$ or ${ }^{3} \mathrm{He}$ gas up to 70 psi. It was used for the determination of target cell ${ }^{3} \mathrm{He}$ and $\mathrm{N}_{2}$ density and the study of $\mathrm{N}_{2}$ and empty dilution factors in the target cell. The reference cell system was designed and constructed by the MIT group.

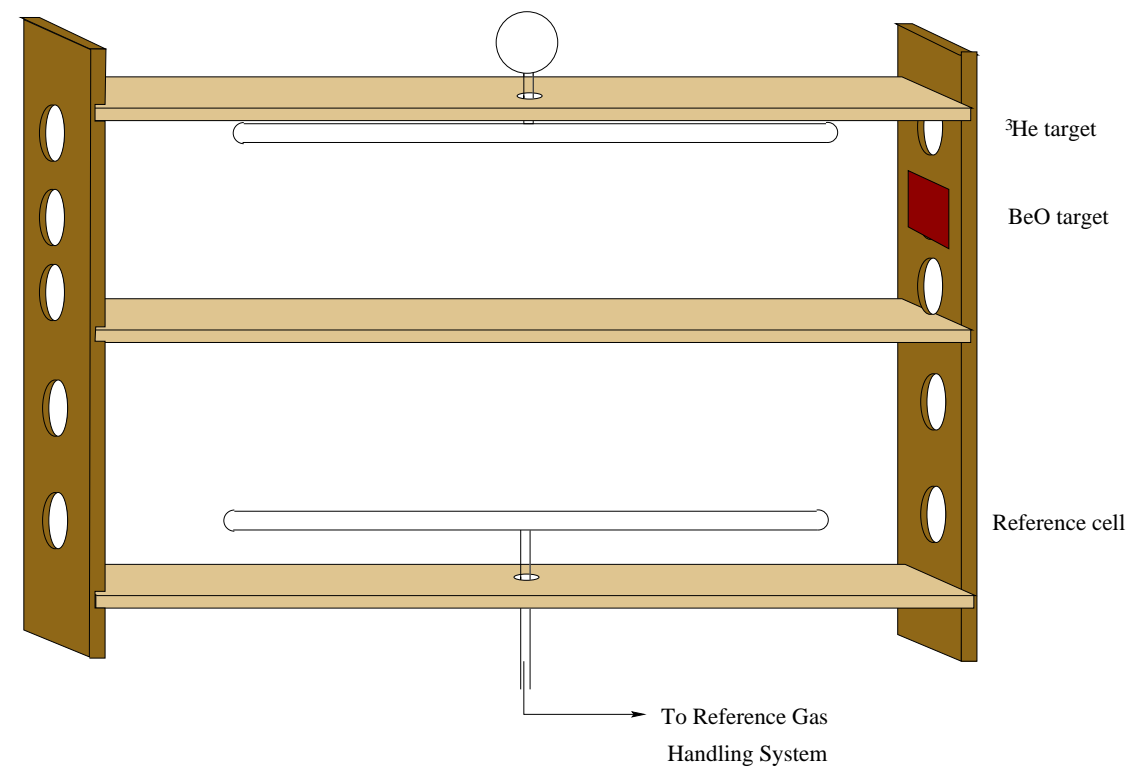

Figure 2-14: Hall A target ladder.

The target ladder was mounted to a Torlon oven. Hot air was passed into the oven through a pipe which was heated with an electric heater. The pumping chamber was placed inside the oven and could be heated to $170-180^{\circ} \mathrm{C}$ to produce $\mathrm{Rb}$ vapor during the experiment. The oven itself was mounted to an electric rod that could move vertically, allowing different targets to be put into the beamline. To reduce 
the electron beam heating, cooling jets of ${ }^{4} \mathrm{He}$ were flew through the entrance and exit windows of the target chamber. The cooling jets system was also designed and constructed by the MIT group. The oven temperature and target cell temperature were monitored using many Resistive Thermal-couple Devices (RTDs) both attached to the target cell and inside the oven.

\subsubsection{Target Polarization Measurement}

As will be discussed in Chapter 3, the product of beam and target polarization can be monitored continuously via elastic polarimetry during the experiment. However, as a cross-check, it is still a good idea to measure the beam and target polarization separately using Møller polarimetry and NMR polarimetry. In this section, we will discuss the NMR polarimetry in detail: the Adiabatic Fast Passage (AFP) technique [60], water calibration and the NMR system.

\section{Adiabatic Fast Passage}

To explain the idea of AFP, let us consider a polarized ${ }^{3} \mathrm{He}$ sample placed in a main holding field in the z-direction, $\mathbf{H}=\mathrm{H}_{0} \hat{\mathbf{k}}$, and apply an oscillating RF field in the x-direction, $\mathbf{H}_{r}=2 \mathrm{H}_{1} \cos \left(\omega_{0} t\right) \hat{\mathbf{i}}$, to the sample.

The RF field can be decomposed into two counter-rotating components, each of magnitude $\mathrm{H}_{1}$ :

$$
\mathbf{H}_{r}=H_{1} \hat{\mathbf{i}}_{+}^{\prime}+H_{1} \hat{\mathbf{i}}_{-}^{\prime}
$$

where $\hat{\mathbf{i}}_{ \pm}^{\prime}=\cos \left(\omega_{0} t\right) \hat{\mathbf{i}} \pm \sin \left(\omega_{0} t\right) \hat{\mathbf{j}}$. To consider the influence of the oscillating field on the magnetization of the polarized ${ }^{3} \mathrm{He}$ sample, it is useful to go to a reference frame rotating around z-axis at $\omega_{0}$, with the $x^{\prime}$-axis along $\hat{\mathbf{i}}^{\prime}=\hat{\mathbf{i}}_{+}^{\prime}$. The effective field in the rotating system is then (Figure 2-15)

$$
\mathbf{H}_{e}=\left(H_{0}-\frac{\omega_{0}}{\gamma}\right) \hat{\mathbf{k}}^{\prime}+H_{1} \hat{\mathbf{i}}^{\prime}
$$

where $\gamma$ is the gyromagnetic ratio for ${ }^{3} \mathrm{He}$. For our experiment, $\frac{\omega_{0}}{\gamma}=28.06 \mathrm{G}$, 


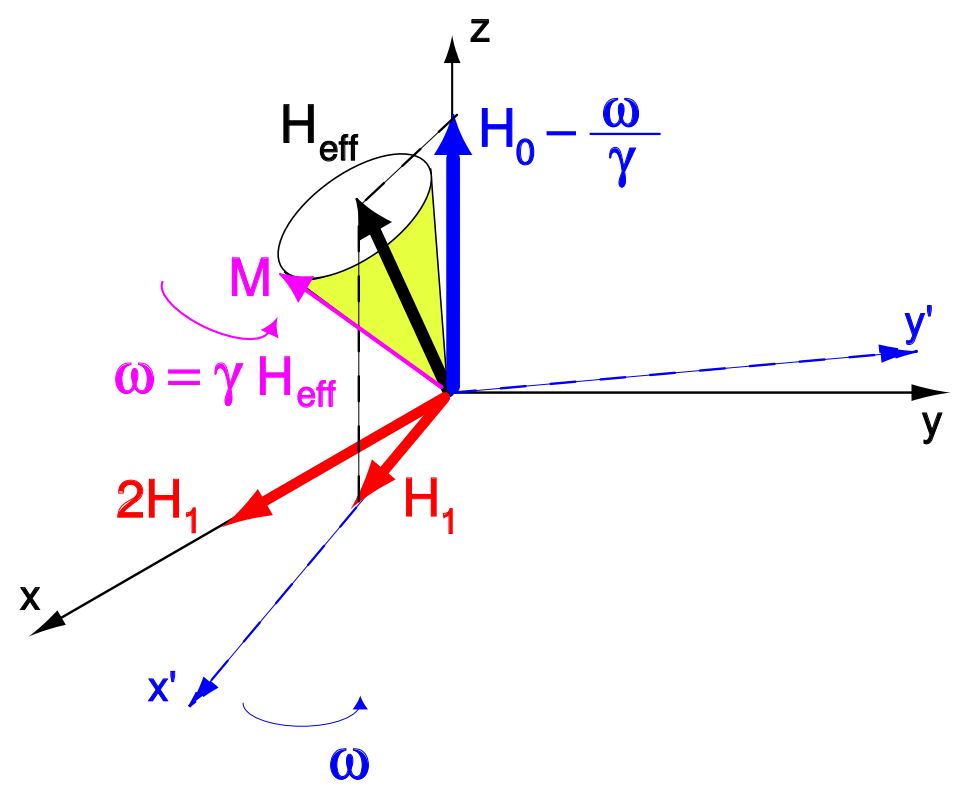

Figure 2-15: Principle of Adiabatic Fast Passage (AFP).

$\mathrm{H}_{1}=90.8 \mathrm{mG}$, and $\mathrm{H}_{0}$ is swept from $25 \mathrm{G}$ to $32 \mathrm{G}$. Initially $\left|H_{0}-\frac{\omega_{0}}{\gamma}\right| \gg H_{1}$, so the effective field is parallel to the z-axis. When $\mathrm{H}_{0}$ increases, the $\mathrm{z}$-component of the effective field becomes smaller and effectively the field is rotating towards the $\mathrm{x}^{\prime}$-axis. If the sweep is slow enough, the ${ }^{3} \mathrm{He}$ magnetization will follow the changing field adiabatically. When $\mathrm{H}_{0}=28.06 \mathrm{G}$, it will be completely aligned with the $\mathrm{x}^{\prime}-$ axis, which is called a resonance. Viewed from the lab frame, the $\mathrm{x}^{\prime}$-component of the ${ }^{3} \mathrm{He}$ magnetization is rotating rapidly around z-axis and will produce a signal in the pick-up coils. The signal peaks at resonance and its size is proportional to the $\mathrm{x}^{\prime}$-axis component of the magnetization:

$$
S \propto \frac{\left\langle M_{3_{H e}}\right\rangle H_{1}}{\sqrt{\left(H_{0}-\frac{\omega_{0}}{\gamma}\right)^{2}+H_{1}^{2}}}
$$

where

$$
\left\langle M_{3^{3} \mathrm{He}}\right\rangle=\mathrm{P}_{3_{\mathrm{He}}} \times \mu_{3_{3} \mathrm{He}} \times\left[{ }^{3} \mathrm{He}\right]
$$


is the average ${ }^{3} \mathrm{He}$ magnetization and is proportional to ${ }^{3} \mathrm{He}$ polarization, the magnetic moment of ${ }^{3} \mathrm{He}$ and the density of ${ }^{3} \mathrm{He}$.

As mentioned above, the sweep rate must be slow enough to make sure the magnetization follows the effective field adiabatically. On the other hand, it must be fast enough so that spin relaxation is not a concern. The condition for adiabatic fast passage is:

$$
\frac{D\left|\Delta H_{0}\right|^{2}}{H_{1}^{2}} \ll \frac{\dot{H}_{0}}{H_{1}} \ll \omega_{0}
$$

where $\mathrm{D}$ is the ${ }^{3} \mathrm{He}$ diffusion rate and $\frac{D\left|\Delta H_{0}\right|^{2}}{H_{1}^{2}}$ is the spin relaxation rate, while $\frac{\dot{H}_{0}}{H_{1}}$ is the rate of change of the effective field near resonance. For our experiment, both conditions were easily met:

$$
\frac{D\left|\Delta H_{0}\right|^{2}}{H_{1}^{2}} \approx 10^{-2}\left[s^{-1}\right] \ll \frac{\dot{H}_{0}}{H_{1}}=24\left[s^{-1}\right] \ll \omega_{0}=5.7 \times 10^{5}\left[s^{-1}\right]
$$

\section{Water Calibration}

In order to extract the polarization of the ${ }^{3} \mathrm{He}$ target, the NMR signal has to be calibrated. This can be done by performing an NMR measurement for a material with known polarization, under exactly the same condition as the NMR measurement for polarized ${ }^{3} \mathrm{He}$ target. The ratio of the ${ }^{3} \mathrm{He}$ polarization to the known material's polarization is proportional to the ratio of the magnitudes of the two NMR signals.

A natural candidate for this calibration is water. This is because when placed in a magnetic field, the protons contained in water will be thermally polarized with the polarization given by the Boltzmann distribution:

$$
P_{w a t e r}^{t h}=\tanh \frac{\mu_{p} B}{k T}
$$

where $\mu_{p}$ is the magnetic moment of the proton, $\mathrm{B}$ is the applied magnetic field, $\mathrm{k}$ is the Boltzmann constant and $\mathrm{T}$ is the temperature. Under the experimental condition the water polarization is about $7 \times 10^{-9}$. 


\section{NMR System}

As can be seen in Figure 2-12, an extra pair of Helmholtz coils (NMR RF Drive Coils) were placed around the target to create a vertical RF field. The frequency of the RF field was determined by a RF generator located in the counting house, and was set to be $91 \mathrm{kHz}$ during the experiment. The magnitude of the RF field was 90.8 $\mathrm{mG}$. The main holding field was swept from 18 to 25 Gauss and then back, at a rate of $1.2 \mathrm{G} / \mathrm{s}$. A pair of pickup coils were placed around the target chamber to pick up the NMR resonance. The plane of the pickup coils was parallel to the vertical RF field to maximize the signal, which was read by a lock-in amplifier referenced to the same RF generator. The whole system was controlled by an Apple computer in the counting house (Figure 2-16).

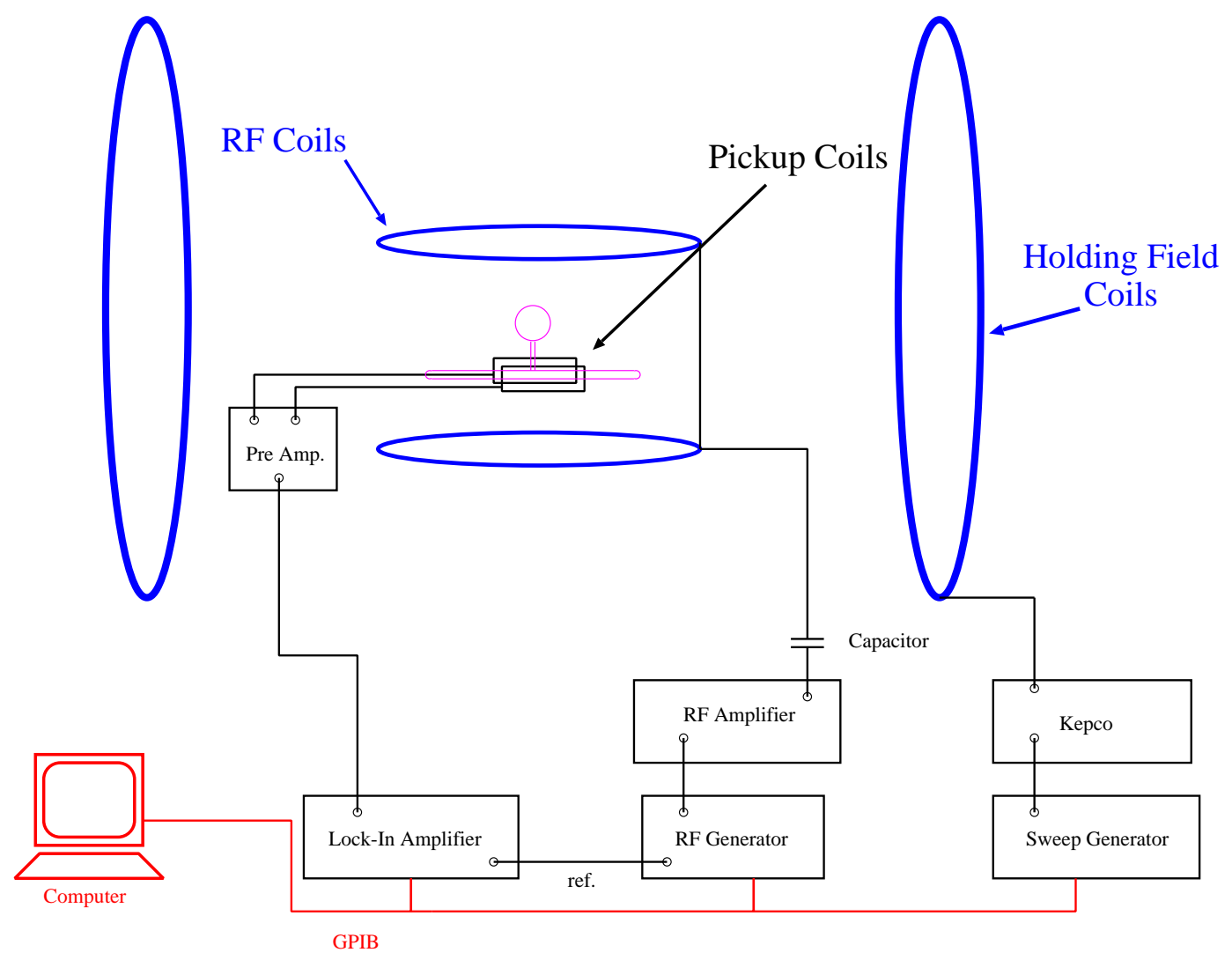

Figure 2-16: Schematics of NMR electronics.

Three NMR measurements were performed on the ${ }^{3} \mathrm{He}$ target every day. The 
water calibration was performed once before the experiment and once after. The average ${ }^{3} \mathrm{He}$ polarization during the experiment was about $30 \%$.

\subsection{High Resolution Spectrometer}

There are two identical High Resolution Spectrometers (HRS) in Hall A, which were designed to achieve high accuracy in the determination of both particle momentum and scattering angle. Looking down the beamline, the one to the left of the electron beam is called the electron arm (HRSe) while the other one is called the hadron arm (HRSh). Each spectrometer consists of three quadrupoles (Q1, Q2, Q3) and one dipole (D) in a QQDQ configuration. Q1 provides dispersive (vertical) focusing while Q2 and Q3 provide transverse (horizontal) focusing. The dipole has a bending angle of $45^{\circ}$ in the vertical direction and is used to accurately select the particle momentum. Its magnetic field increases with the radial distance, providing a natural focusing in the dispersive direction.

The momentum range of the spectrometer is from 0.3 to $4.0 \mathrm{GeV} / \mathrm{c}$ and the main characteristics of the spectrometer are listed in Table 2.2.

\subsection{Detector Packages}

The detector package for the electron arm (HRSe) is shown in Figure 2-17. There are a pair of Vertical Drift Chamber (VDC) to precisely locate the trajectories of the charged particles and determine their momenta, two scintillator planes (S1 and S2) to generate event triggers and provide time-of-flight information, a Gas Čerenkov detector sandwiched between S1 and S2 used for $\mathrm{e}^{-} / \pi^{-}$separation, and finally a lead-glass preshower and shower counter to provide additional $\mathrm{e}^{-} / \pi^{-}$separation. The detector package is similar in HRSh except that there is no preshower. 


\begin{tabular}{c|c}
\hline \hline Momentum Range & $0.3-4.0 \mathrm{GeV} / \mathrm{c}$ \\
\hline Configuration & QQDQ \\
\hline Optical Length & $23.4 \mathrm{~m}$ \\
\hline Momentum Acceptance & $\pm 4.5 \%$ \\
\hline Dispersion (D) & $12.4 \mathrm{~cm} \%$ \\
\hline Radial Linear Magnification (M) & 2.5 \\
\hline D/M & 5 \\
\hline Momentum Resolution (FWHM) & $1 \times 10-4$ \\
\hline Angular Acceptance & $\pm 28 \mathrm{mr}$ \\
Horizontal & $\pm 60 \mathrm{mr}$ \\
Vertical & $6.7 \mathrm{msr}$ \\
Solid Angle: & $5.3 \mathrm{msr}$ \\
\hline (rectangular approximation) & \\
(elliptical approximation) & $0.6 \mathrm{mr}$ \\
\hline Angular Resolution: (FWHM) & $2.0 \mathrm{mr}$ \\
\hline Horizontal & $\pm 5 \mathrm{~cm}$ \\
Vertical & $1.5 \mathrm{~mm}$ \\
\hline Transverse Length Acceptance & $0.1 \mathrm{mr}$ \\
\hline Spectrometer Angle Determination Accuracy &
\end{tabular}

Table 2.2: Hall A High Resolution Spectrometer (HRS) general characteristics.

\subsubsection{Vertical Drift Chamber}

The trajectory of a charged particle is the key element in determining its momentum and reconstructing its target variables. Two paired vertical drift chambers (VDCs) are used in Hall A to determine trajectories of charged particles at the focal plane. From Figure 2-18, it can be seen that these two VDCs are identical and parallel to each other. The bottom one is placed on the actual focal plane, while the top one is about $35 \mathrm{~cm}$ above and is shifted by about $35 \mathrm{~cm}$ with respect to the bottom one in the dispersive direction. The size of each VDC is about $240 \mathrm{~cm} \times 40 \mathrm{~cm} \times 10 \mathrm{~cm}$, with an active area of about $211.8 \mathrm{~cm} \times 28.8 \mathrm{~cm}$.

Each VDC consists of two gas windows, two wire planes (U plane and V plane), and three high voltage (HV) planes. Each gas window is made of Mylar coated with 


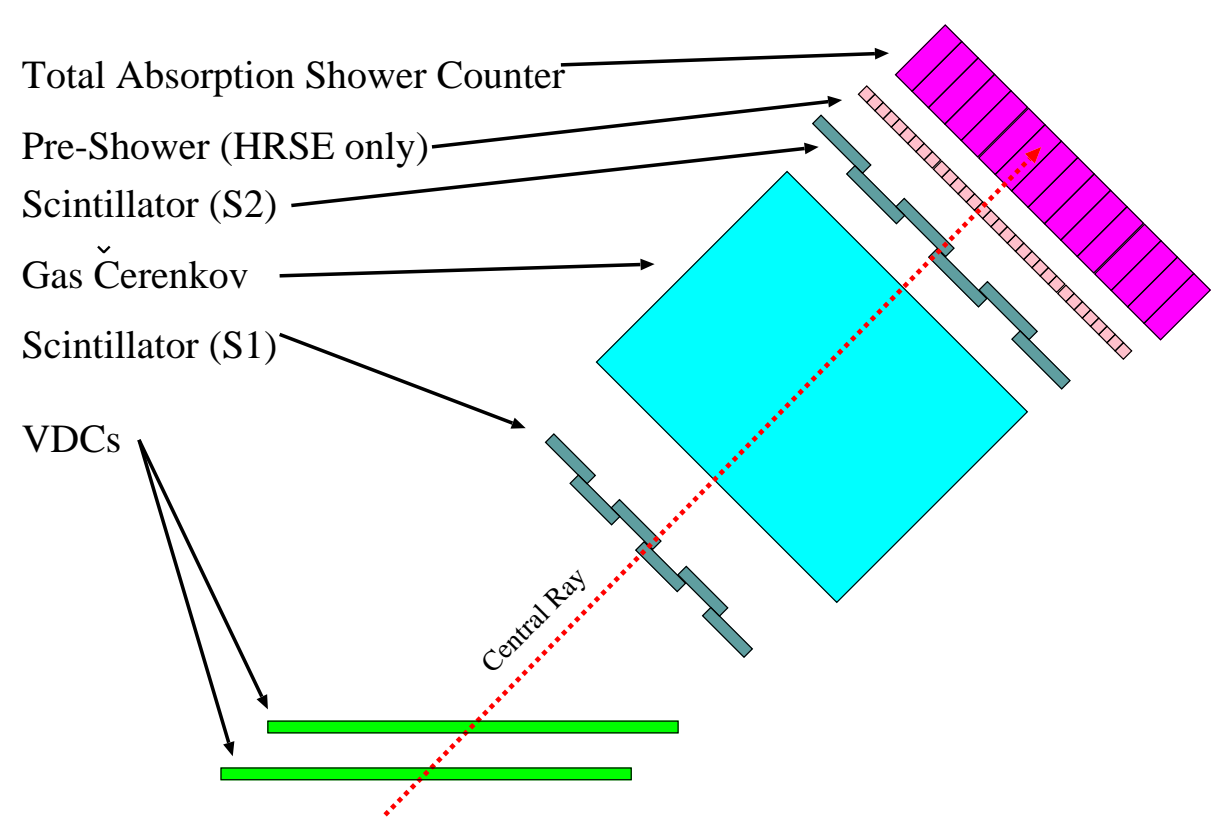

Figure 2-17: Hall A detector package in the electron arm. The detector package in the hadron arm is similar, expect that there is no preshower.

aluminum to shield the signals from noise. The three high voltage planes are made of Mylar coated with gold for good conductivity. Each wire plane is sandwiched between two high voltage planes. The two wire planes are perpendicular to each other and each one has 400 wires. The first and last 16 wires are grounded to shape the electric field and the remaining 368 wires are all $20-\mu \mathrm{m}$ diameter signal wires which are made of tungsten coated with gold. The wires are oriented at $\pm 45^{\circ}$ to the dispersive direction. The VDC is filled with an equal mixture of argon and ethane.

When a charged particle passes through the VDC, the gas atoms along its trajectory will be ionized. The free electrons thus created will drift along the electric field line towards the nearest wire. In the vicinity of a wire, the electric field increases as $1 / \mathrm{r}$, thus the electrons can gain enough energy to ionize other gas atoms. The newly produced free electrons will also drift along the electric field towards the wide and gain enough energy to induce even more ionizations. This process, called avalanche, goes on and on until the electrons collide with the wire and create a negative signal which is fed into a Time-Digital Converter (TDC) and an Analog-Digital Converter 


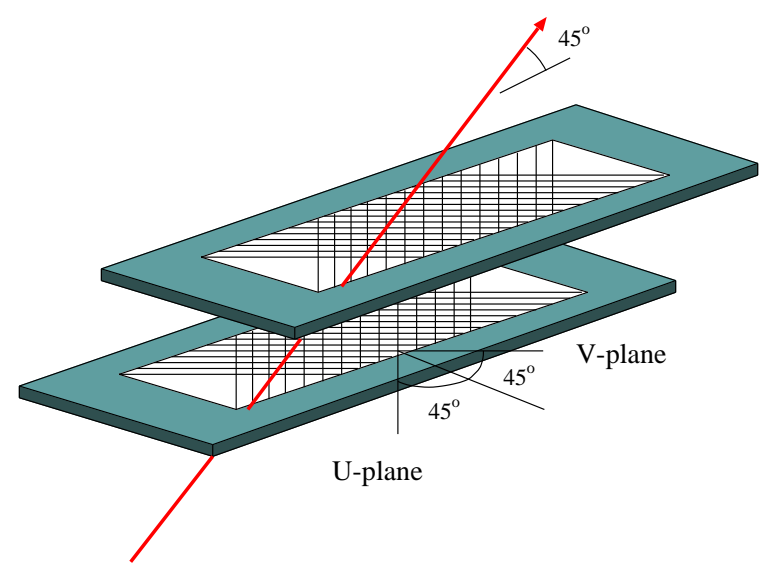

Figure 2-18: Schematics of Hall A VDC wire planes.

(ADC). The time difference between the TDC signal and the trigger signal from the scintillators measures the time elapsed between the initial ionization and the induction of the signal on the wire. With the knowledge of the drift velocity of the particle in the VDC gas this time gap can be used to calculate the shortest drift distance of the particle (called geodetic distance) and eventually the perpendicular distance between the particle trajectory and the wire. Generally five or six adjacent wires fire for one trajectory in each wire plane. From the perpendicular distances between the trajectory and the five or six wires the intersection point between the trajectory and the wire plane can be determined (Figure 2-19). Since there are four wire planes, four intersection points will be obtained which can be used to determine both the position $\left(x_{f p}, y_{f p}\right)$, and the angle $\left(\theta_{f p}, \phi_{f p}\right)$ of the trajectory on the focal plane.

\subsubsection{Scintillator}

Two planes of scintillators (S1 and S2) are used to generate event triggers. Each scintillator is fragmented into 6 paddles with $0.5 \mathrm{~cm}$ overlap between two paddles (Figure 2-20). At each side of a paddle, there is a 2-inch phototube which generates signals that are sent to both an $\mathrm{ADC}$ and a TDC. The active area of S1 is about 170 $\mathrm{cm} \times 35 \mathrm{~cm}$, while the active area of $\mathrm{S} 2$ is about $220 \mathrm{~cm} \times 54 \mathrm{~cm}$. 


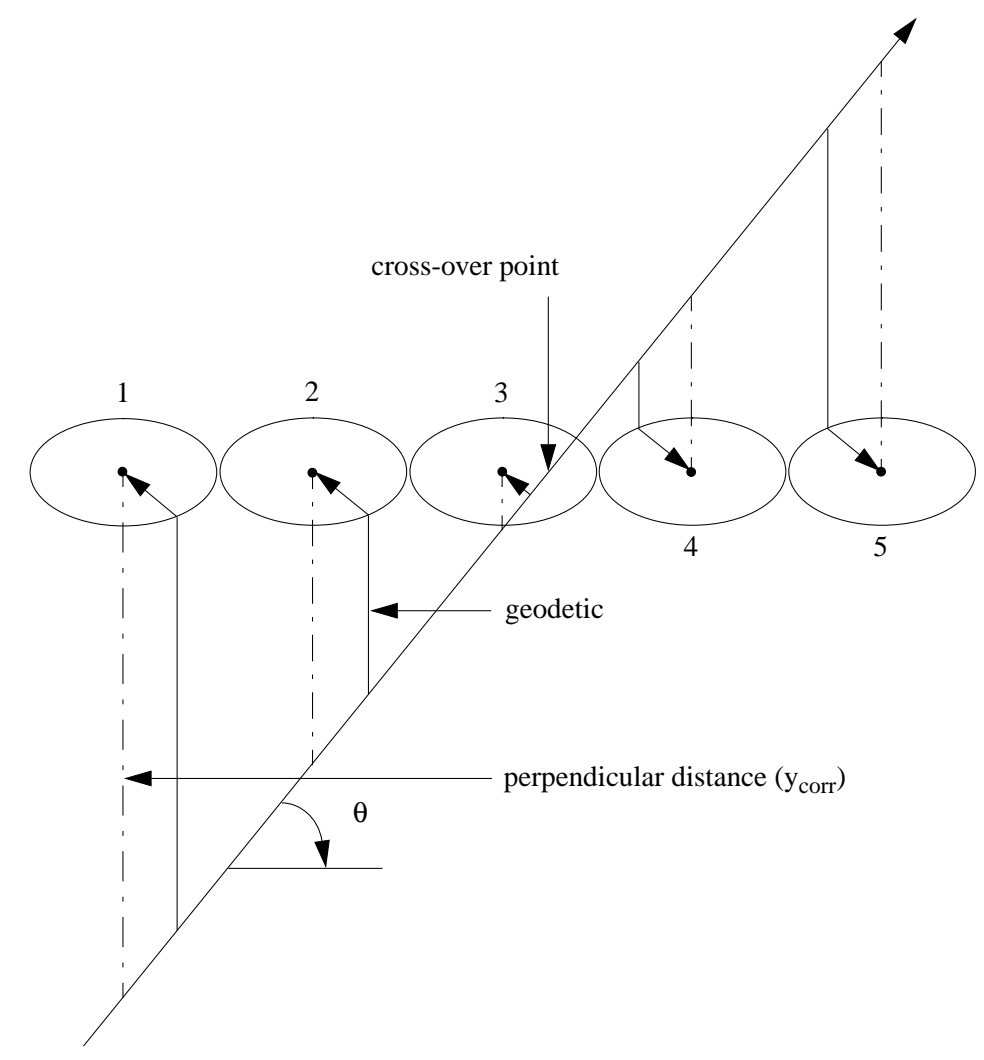

Figure 2-19: A typical trajectory in one of the VDC wire planes. The geodetic is the shortest drift distance and can be used to calculate the perpendicular distance. The intersection point of the particle with the wire plane can be determined with the knowledge of all perpendicular distances from the five fired wires.

\subsubsection{Gas Čerenkov}

The Gas Čerenkov was used to separate electrons from pions. To understand its principle, let us consider a particle traveling in a medium with refractive index $n$. If the particle is moving faster than the speed of light inside the medium, $\beta>\frac{1}{n}$, it will emit light in a forward cone with an apex angle given by $\theta_{c}=\cos ^{-1}\left(\frac{1}{\beta n}\right)$. At same momentum, electrons move faster than pions. So if we select a medium with a refractive index such that $\frac{1}{n}<\beta_{e}$ and $\frac{1}{n}>\beta_{\pi}$, then Čerenkov light will be emitted 


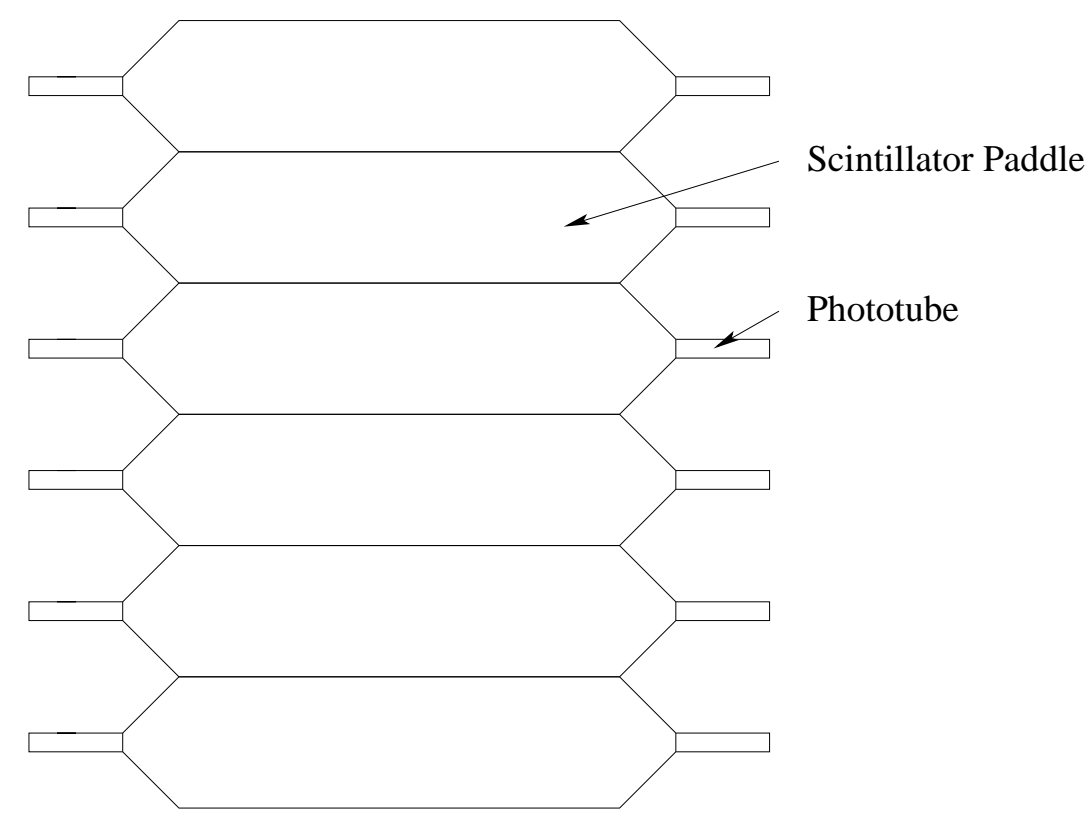

Figure 2-20: Schematics of a scintillator plane. There are six scintillator paddles and one phototube is installed at each side of a paddle.

only if the particle is an electron. By collecting the emitted photons one can thus tell whether a particle is an electron or pion.

The Hall A Čerenkov detector (Figure 2-21) is a rectangular tank filled with 1 atm of $\mathrm{CO}_{2}$, which has a reflective index of $\mathrm{n}=1.00041$, therefore the threshold momentum for electrons to emit Čerenkov light is only $0.017 \mathrm{GeV}$, while the threshold for pions is $4.8 \mathrm{GeV}$. There are ten spherical mirrors in front of the exit window of the detector and five 5-inch photo-multiplier tubes (PMTs) on each side of the detector. Emitted photons are reflected by the mirrors into the PMTs and the photon detection efficiency was found to be larger than $99 \%$ during the experiment.

\subsubsection{Shower Detector}

The shower detector was used in conjunction with Čerenkov detector to reject pions during the experiment. A shower detector is essentially an energy depositor. When a particle passes through some material, it emits Bremsstrahlung radiation along its 


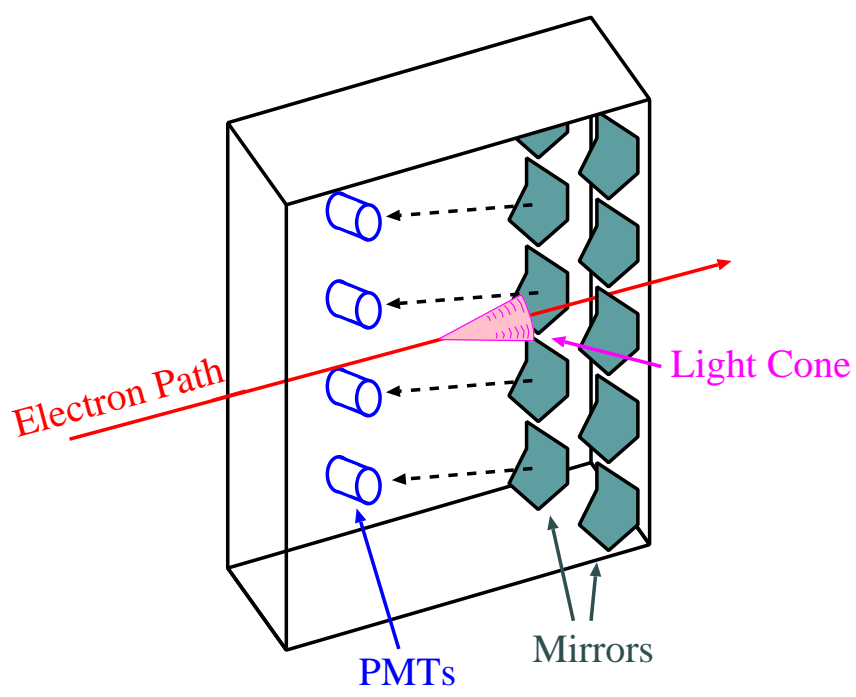

Figure 2-21: Hall A gas Čerenkov detector.

path. This radiation then creates secondary particles which in turn, creates even more particles. This process is called a cascade and in this way part or all of the incoming particle's energy is converted into photons, which can be detected using PMTs. The total amount of photons detected is proportional to the particle energy. A properly calibrated shower detector has an energy over momentum $(\mathrm{E} / \mathrm{p})$ ratio of one for electrons and less than one for pions since pions do not deposit as much energy as electrons. Thus the $\mathrm{e}^{-} / \pi^{-}$separation can be done based on the $\mathrm{E} / \mathrm{p}$ ratio.

The shower detectors for Hall A HRSe and HRSh are shown in Figure 2-22. The HRSe shower detector has two layers. The first layer is called the preshower and is made of $48(24 \times 2)$ blocks of TF-1 lead glass. Each block measures $10 \mathrm{~cm} \times 10 \mathrm{~cm} \times$ $35 \mathrm{~cm}$, and the total radiation length of the preshower is 3.65 . The second layer, the shower counter, is made of $96(16 \times 6)$ blocks of SF-5 lead glass. Each block measures $15 \mathrm{~cm} \times 15 \mathrm{~cm} \times 35 \mathrm{~cm}$ and the total radiation length for the shower counter is 15.2 . The photons are collected by the PMTs attached to both ends of the glass blocks. The HRSh shower detector is made of $32(16 \times 2)$ blocks of SF-5 lead glass, and the total radiation length is about 6 . 


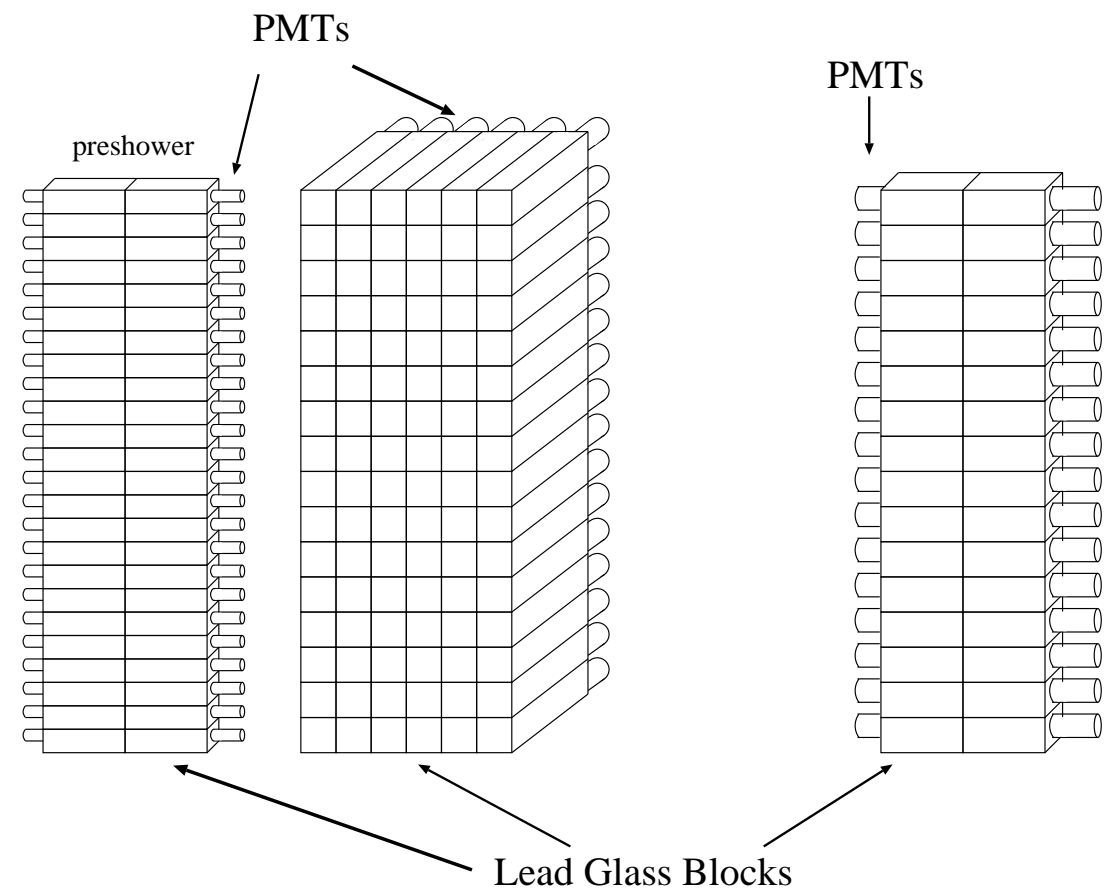

Shower Detector for HRSE

Shower Detector for HRSH

Figure 2-22: Hall A shower detectors.

\subsection{Data Acquisition System}

\subsubsection{Trigger Electronics}

The trigger electronics determines whether an event should be recorded by the data acquisition system. Typically there are five $\mathrm{S}$ triggers and one timing trigger. The $\mathrm{S}$ triggers are generated by the signals from the two sides of each scintillator paddle while the timing trigger is generated by a $1024 \mathrm{~Hz}$ electronic clock. The detailed logic of the $\mathrm{S}$ trigger electronic is shown in Figure 2-23 for the electron arm and Figure 2-24 for the hadron arm.

Only a certain hit pattern, called S-ray, is taken as a good trigger. The S-ray is defined in the following manner: if paddle $\mathrm{n}$ of one scintillator plane fires, then on the other scintillator plane, there must be a hit from one of the three paddles $n-1, n$, $\mathrm{n}+1$, or the overlap between two of them. The $\mathrm{S}$ triggers are further divided into five 

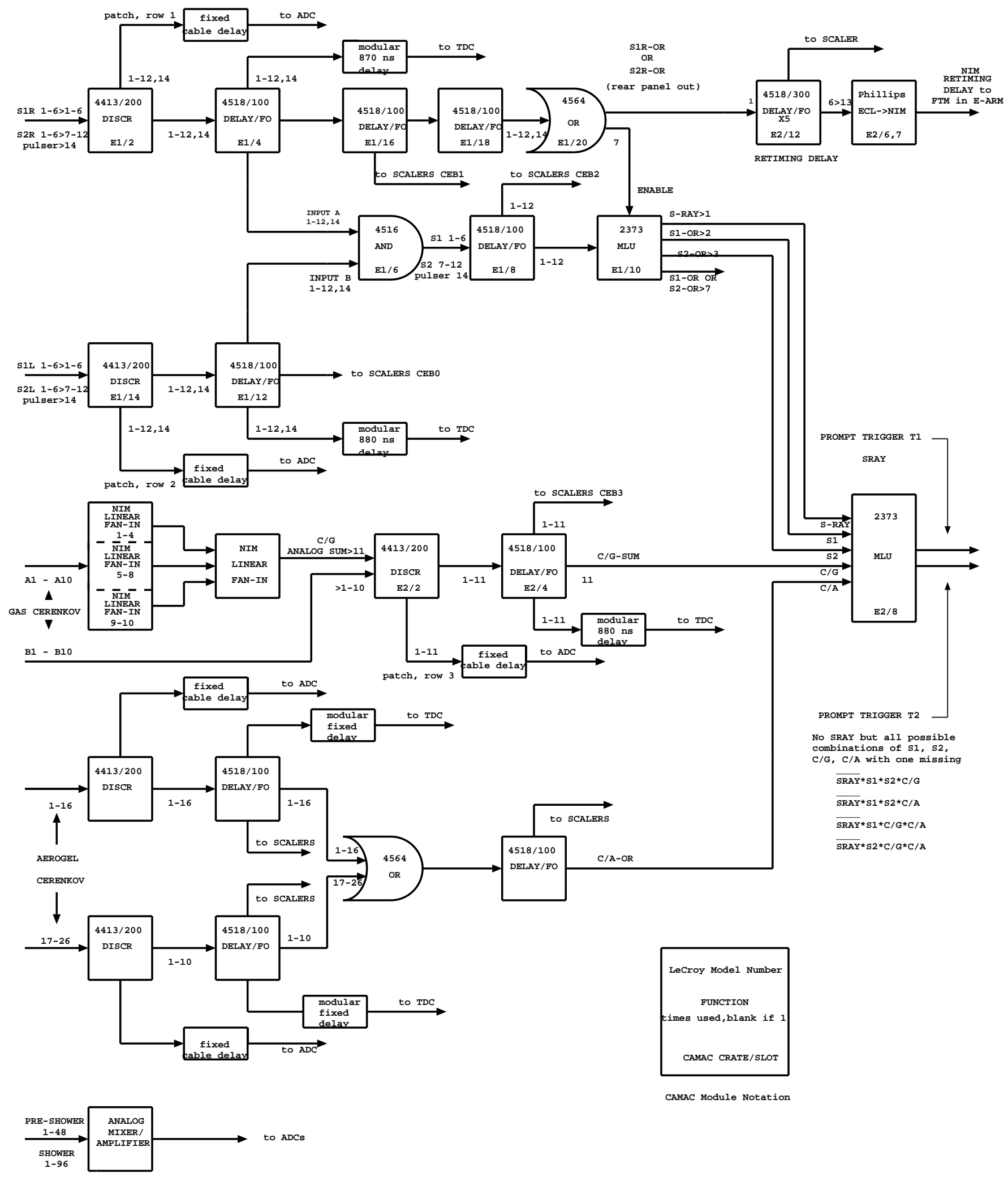

HALL A ELECTRON PROMPT TRIGGER DESIGN 11/13/97

Figure 2-23: Hall A electron arm trigger electronics. 


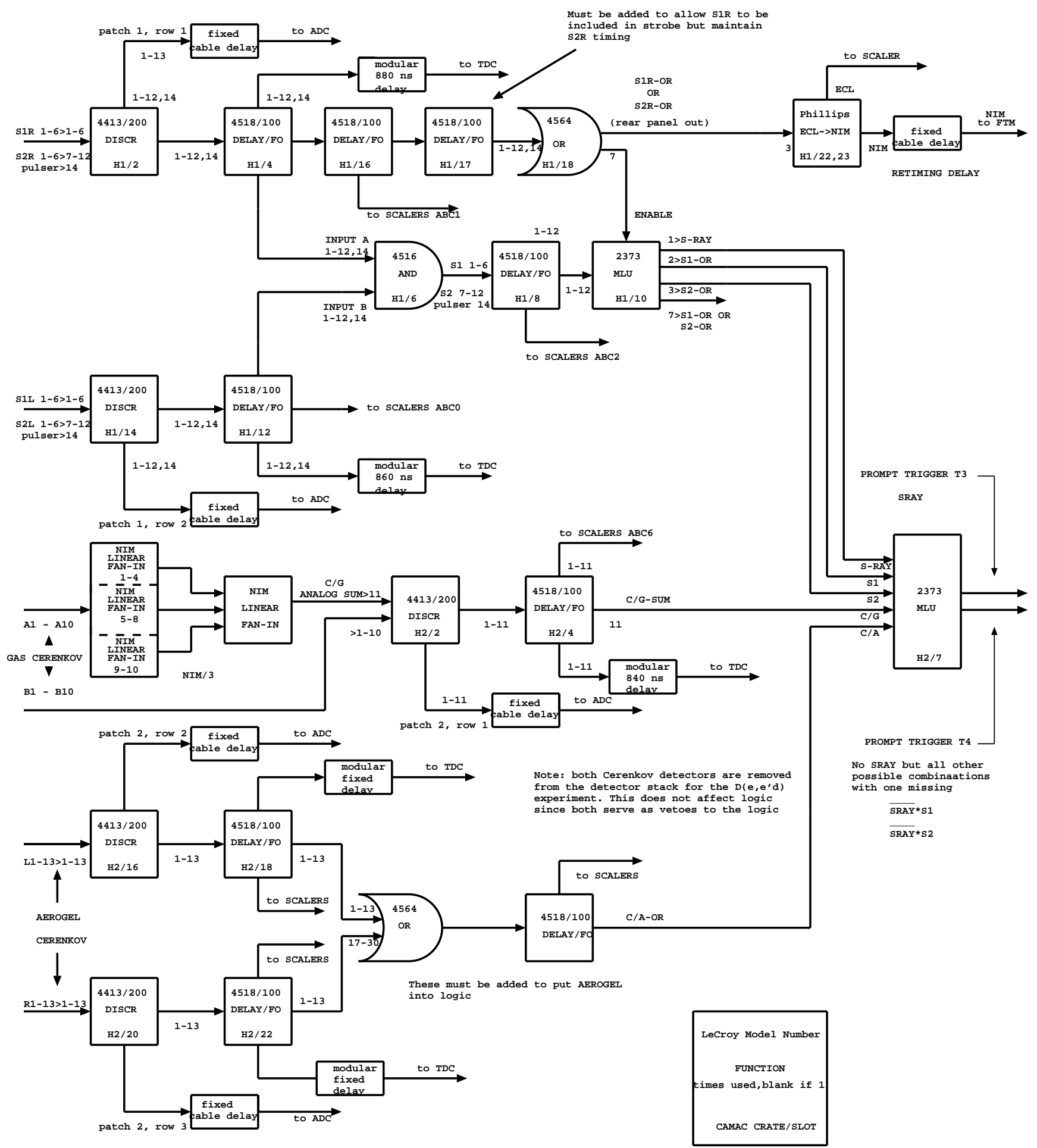

Note: The FPP is not included in TRIGGER LOGIC

Note: The modular delays are NIM LD1200

HALI A HADRON PROMPT TRIGGER DESIGN 11/13/97

Figure 2-24: Hall A hadron arm trigger electronics. 
different kinds depending on the S-ray logic and some other criteria:

- S1 trigger: both scintillator planes fire and a S-ray logic is satisfied in the electron arm.

- S2 trigger: only one of the scintillator planes and Gas Čerenkov detector fire in the electron arm.

- S3 trigger: same as $\mathrm{S} 1$, but in the hadron arm.

- S4 trigger: same as S2, but in the hadron arm.

- S5 trigger: AND of S1 and S3 signal in a certain timing gate from the timing signal.

S1, S3 and S5 triggers are taken to be good events, while S2 and S4 triggers are used to study scintillator inefficiencies. All triggers are sent to scalers to be recorded and are also sent to the Trigger Supervisor (TS). The TS synchronizes the readout crates, administers the deadtime logic of the entire system, and prescales the $\mathrm{S}$ triggers.

\subsubsection{Data Acquisition}

The Hall A Data Acquisition System is shown in Figure 2-25. For each spectrometer, there are one VME crate, one Fastbus crate and one Trigger Supervisor, all located inside the spectrometer shielding house. All TDCs and ADCs are Fastbus based, because Fastbus electronics provides higher data acquisition rates than traditional CAMAC electronics. The scalers are fed into the VME crate.

The software to control data acquisition is called CODA (CEBAF Online Data Acquisition), a toolkit developed at JLab by the Data Acquisition Group. A HP-9000 computer in the counting house was used to run CODA. There are three components to CODA: 


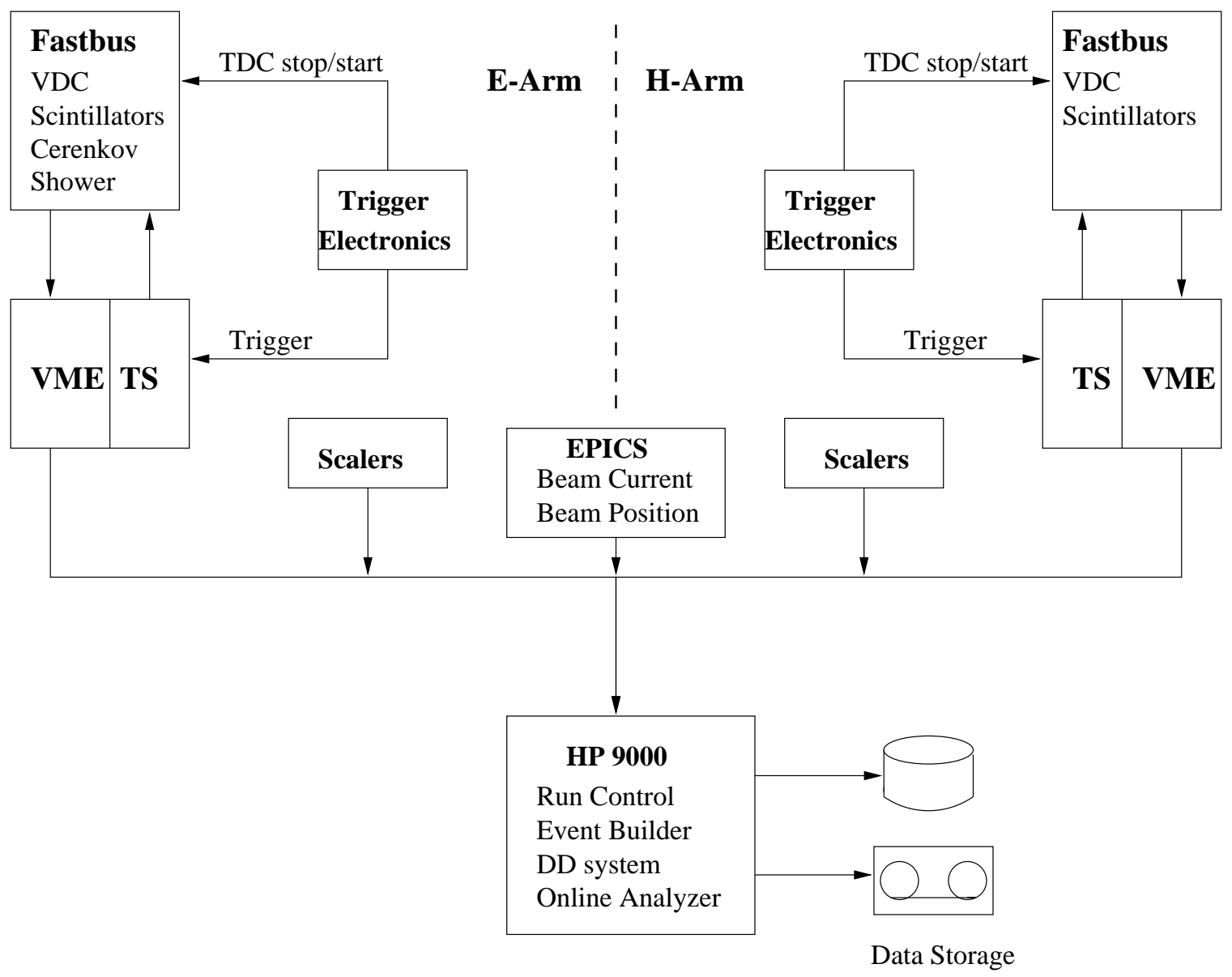

Figure 2-25: Hall A Data Acquisition System.

- Readout Controllers (ROCs) which provide interfaces with the detector system. In this experiment, ROCs were some CPUs in the Fastbus and VME crates. The Trigger Supervisor controls the state of each run, and generates the triggers that cause ROCs to be read out.

- An Event Builder (EB) which collects all ROC data fragments and incorporates necessary CODA header information to build an event.

- An Analyzer/Data Distribution (ANA/DD) which analyzes and sends the events to hard disks of computers in the counting house and later transfers them to 
main storage.

Most events are physics events, which record important variables to reconstruct the scattering process. There are also a few special events. If the status of a run is changed from a previous run, a series of status events will be written to the beginning of the data file for that run. In addition, the scaler event and the EPICS event, which includes the beam current and beam position information and contains certain information on the polarized ${ }^{3} \mathrm{He}$ target, are written to the data stream every few seconds. 


\section{Chapter 3}

\section{Data Analysis}

In this chapter we will mainly focus on the analysis of the HRSh data to get the spin-dependent asymmetry in the threshold region of ${ }^{3} \mathrm{He}\left(\vec{e}, e^{\prime}\right)$. One of the major advantages of an asymmetry measurement is that it is much less dependent on the usual systematic uncertainties associated with a cross-section measurement, such as detector inefficiencies, target density, beam charge, data acquisition (DAQ) deadtime and spectrometer acceptance, and thus produces a very clean physics signal. However, at the end of the chapter we will also present the analysis of the experimental cross section and compare it with a state-of-the-art Faddeev calculation.

\subsection{Overview of Asymmetry Analysis}

The experimental raw asymmetry is calculated as

$$
A^{e x p}=\frac{N_{+}-N_{-}}{N_{+}+N_{-}}
$$

where $\mathrm{N}_{+}$and $\mathrm{N}_{-}$are the electron yields normalized by charge and electronic live time for positive and negative electron helicities, respectively.

To get the physics asymmetry, we have to correct for the beam and target po- 
larizations, subtract the empty target and $\mathrm{N}_{2}$ background and elastic radiative tail contribution and then perform the radiative correction on the quasielastic asymmetry. Finally, since the experimental asymmetry is always formed over a certain energy bin and averaged over the spectrometer acceptance, while the theoretical calculation for the physics asymmetry is performed at each energy point for the central kinematics, we also have to correct for the bin-averaging and acceptance effects.

The exact formula can be written as:

$$
\begin{aligned}
A= & \left(1+R^{e r t}\right)\left(1+R^{e m p}+R^{N_{2}}\right) \frac{A^{e x p}}{P_{b} P_{t}} \\
& -\left(1+R^{e r t}\right) R^{e m p} \frac{A^{e m p}}{P_{b} P_{t}}-\left(1+R^{e r t}\right) R^{N_{2}} \frac{A^{N_{2}}}{P_{b} P_{t}} \\
& -R^{e r t} A^{e r t}+\Delta A^{q e}+\Delta A^{\text {bin }}
\end{aligned}
$$

where $\mathrm{P}_{b} \mathrm{P}_{t}$ is the product of beam and target polarizations, $\mathrm{R}^{e m p}$ and $\mathrm{A}^{e m p}$ are the dilution factor and false asymmetry for the empty cell background, $\mathrm{R}^{N_{2}}$ and $\mathrm{A}^{N_{2}}$ are the dilution factor and false asymmetry for the $\mathrm{N}_{2}$ background, $R^{\text {ert }}$ and $\mathrm{A}^{\text {ert }}$ are the dilution factor and asymmetry for the elastic radiative tail, $\Delta A^{q e}$ is the radiative correction to the quasielastic asymmetry, and $\Delta A^{\text {bin }}$ is the correction for bin-averaging and acceptance effects.

Ideally the empty target and $\mathrm{N}_{2}$ false asymmetries should be consistent with zero, and we will perform this check for each kinematics. After the consistency check is done, the physics asymmetry can be calculated as

$$
\begin{aligned}
A= & \left(1+R^{e r t}\right)\left(1+R^{e m p}+R^{N_{2}}\right) \frac{A^{e x p}}{P_{b} P_{t}} \\
- & R^{\text {ert }} A^{\text {ert }}+\Delta A^{q e}+\Delta A^{b i n}
\end{aligned}
$$

Among these factors, $\mathrm{A}^{e x p}, \mathrm{R}^{e m p}, \mathrm{~A}^{e m p}, \mathrm{R}^{N_{2}}, \mathrm{~A}^{N_{2}}$ can be determined directly from data, while $R^{e r t}, \mathrm{~A}^{e r t}, \Delta A^{q e}$ and $\Delta A^{\text {bin }}$ have to be determined using a simulation program. As previously mentioned, $\mathrm{P}_{b} \mathrm{P}_{t}$ was monitored continuously during the ex- 
periment via elastic polarimetry, and is determined as the ratio between the measured elastic asymmetry and the simulated elastic asymmetry. We will discuss each of the above factors in the following sections.

\subsection{Experimental Raw Asymmetry}

As was previously mentioned, the HRSh data covered both the elastic peak and the threshold region and were taken at two kinematics with $\mathrm{Q}^{2}$-values of $0.1(\mathrm{GeV} / \mathrm{c})^{2}$ and $0.2(\mathrm{GeV} / \mathrm{c})^{2}$, respectively.

The elastic data were used as elastic polarimetry to determine the product of the beam and target polarizations. The raw elastic asymmetry was formed by

$$
A^{e l}=\frac{N_{+}^{e l}-N_{-}^{e l}}{N_{+}^{e l}+N_{-}^{e l}}
$$

where $\mathrm{N}_{+}^{e l}$ and $\mathrm{N}_{-}^{e l}$ are the electron yields normalized by charge and electronic live time for positive and negative electron helicities, and averaged over a $2-\mathrm{MeV}$ bin from $-1 \mathrm{MeV}$ to $1 \mathrm{MeV}$ in the excitation energy spectrum.

The raw quasielastic asymmetry in the threshold region was calculated in a similar way, but averaged over $5-\mathrm{MeV}$ bins. Thus at $\mathrm{Q}^{2}=0.1(\mathrm{GeV} / \mathrm{c})^{2}$, there were six data points from $5.5 \mathrm{MeV}$ to $35.5 \mathrm{MeV}$ in the excitation energy spectrum, while at $\mathrm{Q}^{2}=$ $0.2(\mathrm{GeV} / \mathrm{c})^{2}$, there were 9 data points from $5.5 \mathrm{MeV}$ to $50.5 \mathrm{MeV}$.

For asymmetry measurements, there can be various false asymmetries due to helicity-correlated variations in the experimental system, such as helicity-correlated beam current shift, helicity-correlated beam position shift, helicity-correlated detector inefficiencies, or non-helicity-correlated empty target false asymmetry and $\mathrm{N}_{2}$ false asymmetry. To minimize the effect of these false asymmetries, the experimental data were taken with different settings by flipping the target polarization direction and taking a half-wave plate in and out to change the beam helicity. The basic premise is that while the physics asymmetry should always change sign whenever the target 
polarization is flipped or the beam wave plate status is changed, the false asymmetries need not to (the helicity-correlated false asymmetries should not change sign when the target polarization is flipped, while the non-helicity-correlated false asymmetries should not change sign in either case). Thus by combining the asymmetries from the four different settings, with their signs reversed when necessary, the false asymmetries will largely cancel out. On the other hand, it is still necessary to understand various false asymmetries and study systematic uncertainties due to them.

The target polarization angle with respect to the beam line, $\theta_{\text {spin }}$, was initially set at $-62.5^{\circ}$, and could be rotated to $-243.6^{\circ}$ and back through a magnetic field rotation program during the experiment. The flipping angle was not exactly $180^{\circ}$ due to an offset in the rotation program. At $\theta_{\text {spin }}=-62.5^{\circ}$, the corresponding target polarization angle with respect to the three-momentum transfer, $\theta^{*}$, varied from $131.2^{\circ}$ to $136.5^{\circ}$ for $Q^{2}=0.1(\mathrm{GeV} / \mathrm{c})^{2}$, and from $134.2^{\circ}$ to $140.0^{\circ}$ for $Q^{2}=0.2$ $(\mathrm{GeV} / \mathrm{c})^{2}$. At $\theta_{\text {spin }}=-243.6^{\circ}, \theta^{*}$ varied from $42.4^{\circ}$ to $47.7^{\circ}$ for $Q^{2}=0.1(\mathrm{GeV} / \mathrm{c})^{2}$, and from $38.9^{\circ}$ to $44.7^{\circ}$ for $Q^{2}=0.2(\mathrm{GeV} / \mathrm{c})^{2}$. At these $\theta^{*}$ ranges, it was found that the contribution to the spin-dependent asymmetry from $R_{T L^{\prime}}$ was comparable to that from $R_{T^{\prime}}$.

The raw quasielastic asymmetries for the four different settings at $\mathrm{Q}^{2}=0.1(\mathrm{GeV} / \mathrm{c})^{2}$ are shown in Figure 3-1. The elastic asymmetry is also shown as the leftmost point at zero excitation energy. In Figure 3-2 the asymmetries at the four different settings are multiplied by their signs and plotted together. The difference between these asymmetries is mainly due to the change in the product of beam and target polarizations from run to run. For example, the asymmetries with the beam wave plate OUT are consistently higher than those with beam wave plate IN at almost all points, which suggests that the product of beam and target polarizations, $P_{b} P_{t}$, was higher when the beam wave plate was taken out during the experiment. To confirm this, we plot the ratios of the quasielastic asymmetry to the elastic asymmetry, which are not affected by $P_{b} P_{t}$, for different settings in Figure $3-3$. As can be seen, $A_{q e} / A_{e l}$ for different 
settings agree with each other within the statistical error at all data points, which confirms our intuition that the difference in Figure 3-2 is only due to the difference in $P_{b} P_{t}$.

\subsection{Background Subtraction}

\subsubsection{Empty Target and $\mathrm{N}_{2}$ Dilution Factors}

To minimize the empty target contribution, a study of the empty target yield versus the beam position was carried out prior to the experiment and five beam positions relative to the target center were studied. The results are shown in Table 3.1. It can be seen that the beam position with $\mathrm{X}=0 \mathrm{~mm}$ and $\mathrm{Y}=0 \mathrm{~mm}$ gave the lowest empty target rate, and this was set to be the nominal beam center during the experiment.

\begin{tabular}{|c|c|c|c|}
\hline \hline $\mathrm{X}(\mathrm{mm})$ & $\mathrm{Y}(\mathrm{mm})$ & $\mathrm{T}_{1}(\mathrm{~Hz})$ & $\mathrm{T}_{3}(\mathrm{~Hz})$ \\
\hline 0.0 & 0.0 & 178.8 & 87.9 \\
1.5 & 0.0 & 189.5 & 99.8 \\
-1.5 & 0.0 & 311.5 & 131.6 \\
0.0 & 1.5 & 390.0 & 176.8 \\
0.0 & -1.5 & 186.0 & 94.7 \\
\hline \hline
\end{tabular}

Table 3.1: Empty target yields versus beam positions

The background from the two target cell windows can be substantial during the experiment. At $\mathrm{Q}^{2}=0.1(\mathrm{GeV} / \mathrm{c})^{2}$, the two windows were out of spectrometer acceptance, so this was not a big concern. However at $\mathrm{Q}^{2}=0.2(\mathrm{GeV} / \mathrm{c})^{2}$, the two windows were observed in the y-spectrum, and a software cut on y had to be applied to exclude the window contribution.

To get the empty and $\mathrm{N}_{2}$ dilution factors, several runs were taken with empty reference cell and $\mathrm{N}_{2}$ reference cell as the target for each kinematics during the experiment. The empty dilution factor was then calculated as the ratio of the empty 

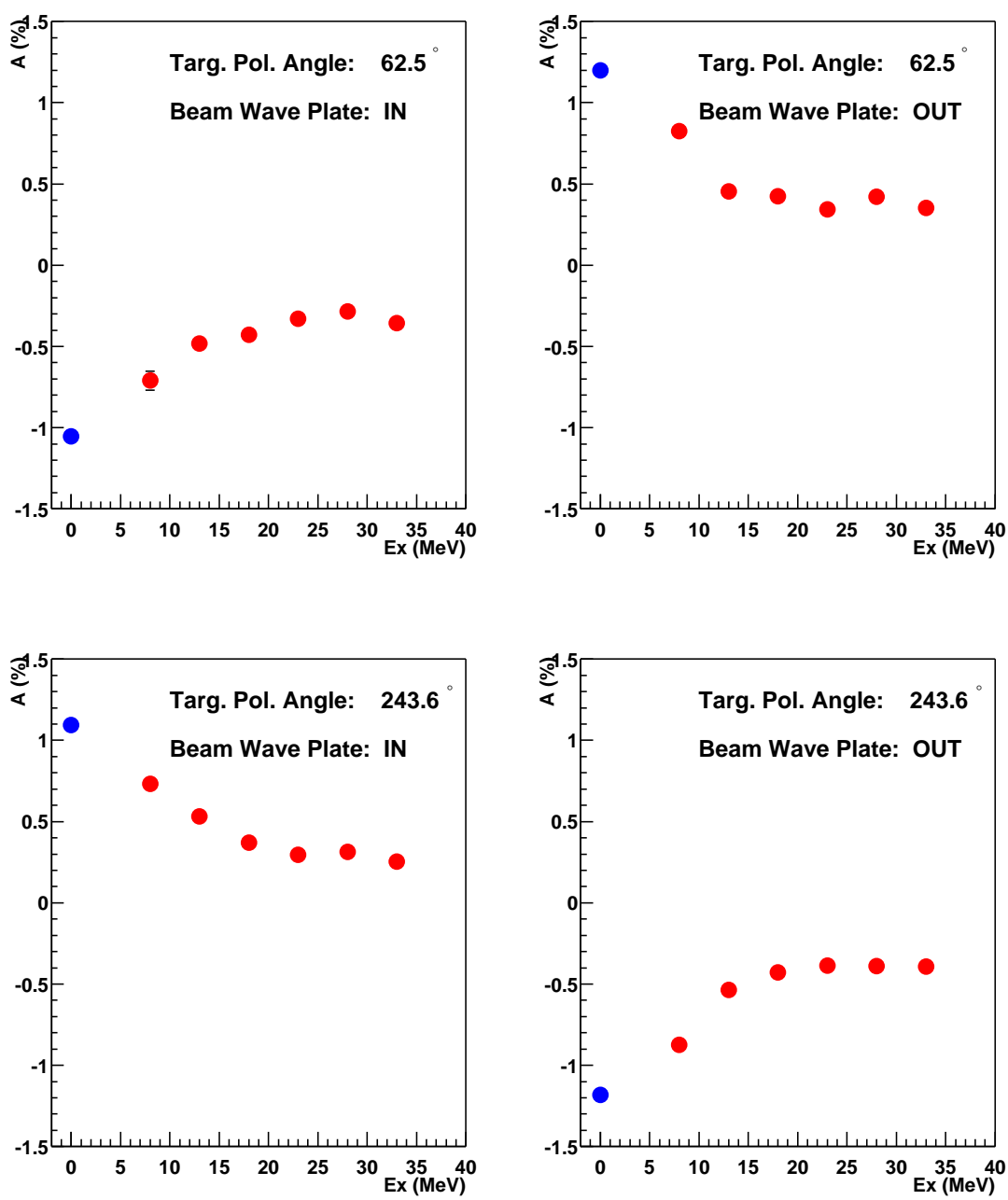

Figure 3-1: Raw asymmetries for different target polarization angle and beam halfwave plate settings at $\mathrm{Q}^{2}=0.1(\mathrm{GeV} / \mathrm{c})^{2}$. The leftmost point is the elastic asymmetry while the rest are quasielastic asymmetry. 


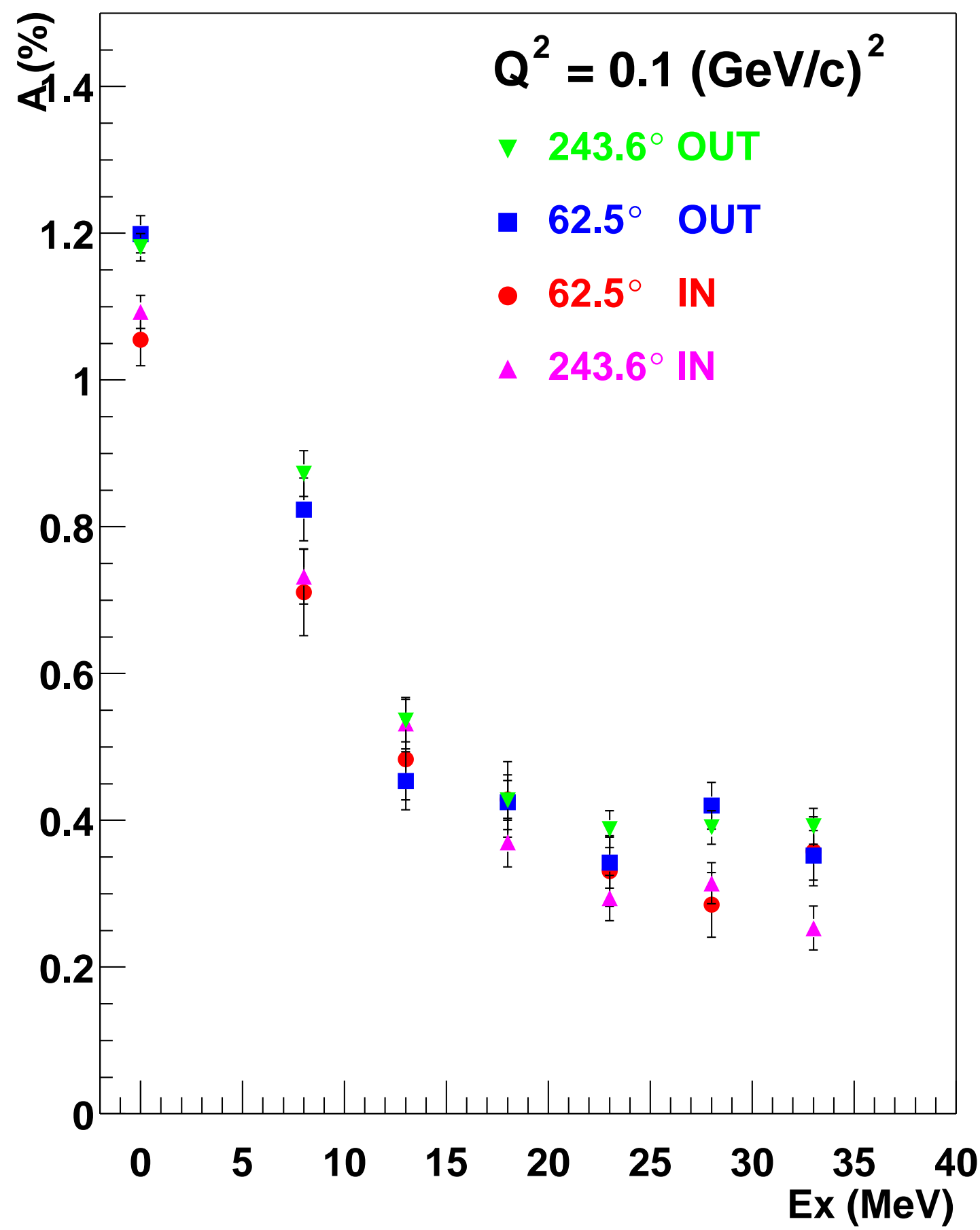

Figure 3-2: Raw asymmetries for different target polarization angle and beam halfwave plate settings at $\mathrm{Q}^{2}=0.1(\mathrm{GeV} / \mathrm{c})^{2}$, multiplied by their signs. The leftmost point is the elastic asymmetry while the rest are quasielastic asymmetry. The difference between these four settings are mainly due to the change in the product of beam and target polarization from run to run. 


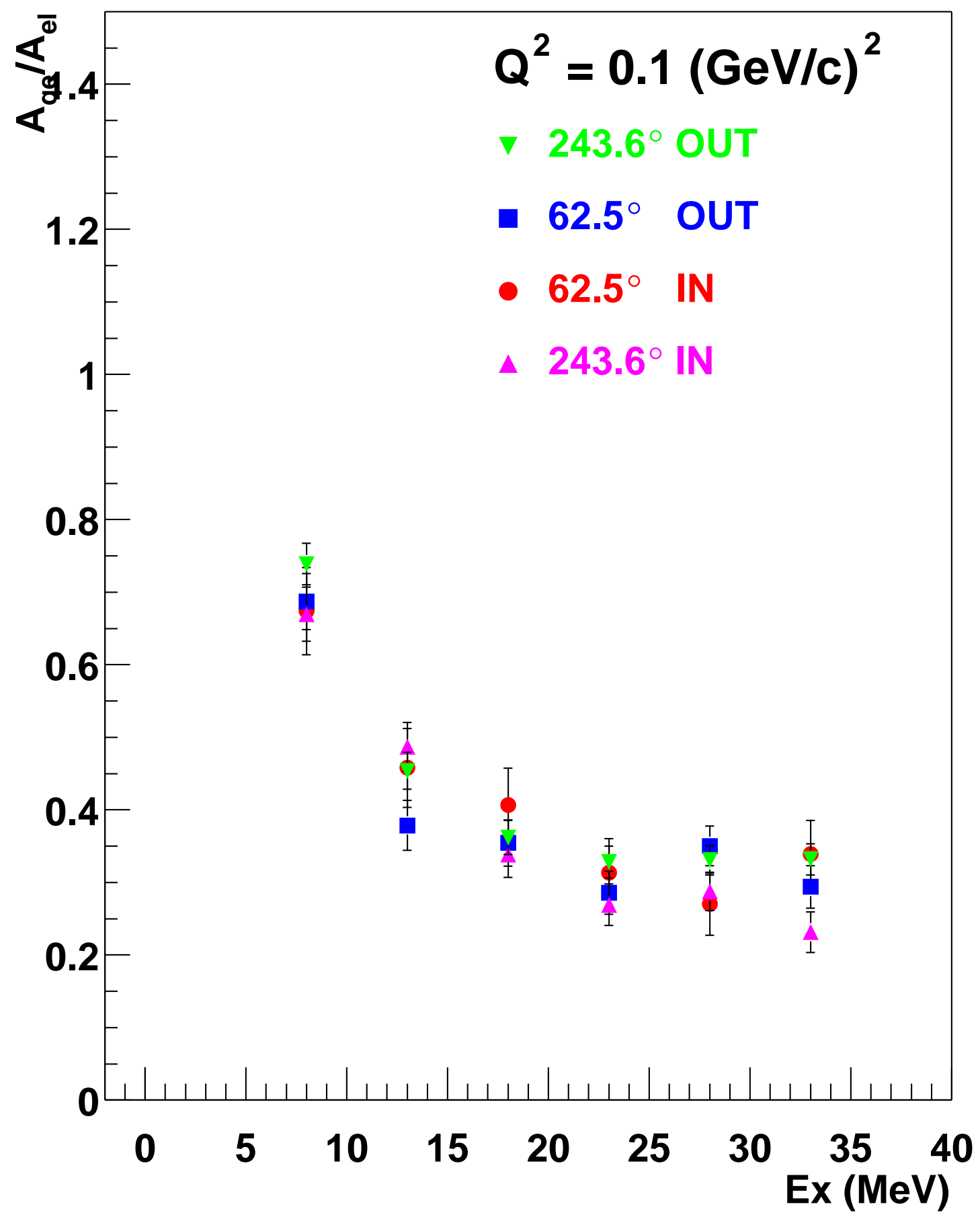

Figure 3-3: The ratios of the quasielastic asymmetry to the elastic asymmetry for different target polarization angle and beam half-wave plate settings at $\mathrm{Q}^{2}=$ $0.1(\mathrm{GeV} / \mathrm{c})^{2}$. 
cell yield to the pure ${ }^{3} \mathrm{He}$ yield, corrected by charge and deadtime. The $\mathrm{N}_{2}$ dilution factor was obtained in a similar way, with the additional knowledge of the $\mathrm{N}_{2}$ pressure inside the target cell, which was obtained through a method called pressure study.

\section{$\mathbf{N}_{2}$ Pressure Study}

The exact $\mathrm{N}_{2}$ pressure inside the target cell was not known very well when the cell was constructed. Also, due to the temperature gradient from the pumping chamber to the target chamber, the $\mathrm{N}_{2}$ pressure during the experiment was different from that under normal temperature. To determine the $\mathrm{N}_{2}$ pressure inside the target cell during the experiment, some reference $\mathrm{N}_{2}$ runs were taken for each kinematics. By comparing the $N_{2}$ elastic peak from the reference cell spectrum to that from the target cell spectrum, the $N_{2}$ pressure can be determined as follows

$$
P_{t g t}=P_{r e f} \times \frac{N_{t g t}^{p e a k}}{N_{r e f}^{p e a k}}
$$

where $P_{t g t}$ is the $\mathrm{N}_{2}$ pressure inside the target cell, $P_{\text {ref }}$ is the $\mathrm{N}_{2}$ pressure inside the reference cell, while $N_{t g t}^{\text {peak }}$ and $N_{r e f}^{\text {peak }}$ are the $N_{2}$ elastic peak yields from the target spectrum and the reference spectrum, respectively, corrected by charge and deadtime. A comparison between these two spectra is shown in Figure 3-4. The leftmost peak is the $\mathrm{N}_{2}$ elastic peak and the slight shift of the peak position between these two runs is due to the difference in energy loss. The two peaks were not centered at zero excitation energy due to an offset in the spectrometer central momentum setting, which was later corrected. Both elastic peaks were shifted to zero excitation energy before taking their ratio.

The $\mathrm{N}_{2}$ pressure inside the target cell was determined in this way to be $2.2 \pm 0.05$ psi, which was confirmed in another independent analysis [79].

\section{Dilution Factors}

With the knowledge of $\mathrm{N}_{2}$ Pressure inside ${ }^{3} \mathrm{He}$ target cell, the dilution factors were obtained in the following way. To improve statistics, all empty runs in the 
Run-1493



N2 component in target cell corrected for empty yield

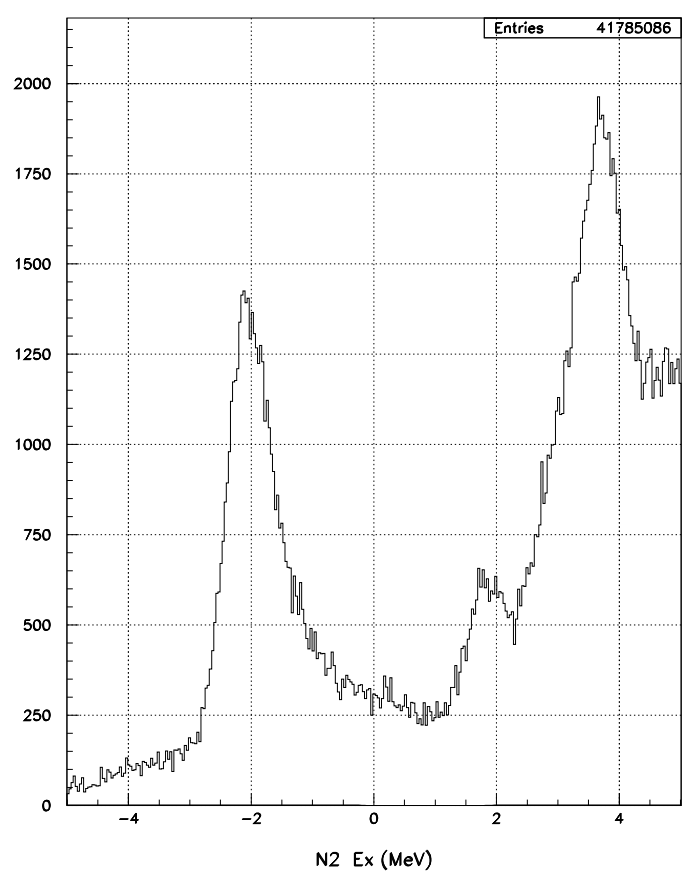

Figure 3-4: $\mathrm{N}_{2}$ excitation energy spectra for a typical reference $\mathrm{N}_{2}$ run (above) and a target run (below). The leftmost peak is the $\mathrm{N}_{2}$ elastic peak and the slight shift of the peak position between these two runs is due to the difference in energy loss. The two peaks were not centered at zero excitation energy due to an offset in the spectrometer central momentum setting, which was later corrected. Both elastic peaks were shifted to zero excitation energy before taking their ratio. 
same kinematics were added together, corrected by charge and deadtime, and then divided by the total empty run number to form a pure empty spectrum. For each $\mathrm{N}_{2}$ run in the same kinematics, the pure empty spectrum was subtracted from the $\mathrm{N}_{2}$ spectrum, also corrected by charge and deadtime. All $\mathrm{N}_{2}$ spectra after this treatment were then normalized to the $\mathrm{N}_{2}$ pressure inside the target cell and averaged to form a pure $\mathrm{N}_{2}$ spectrum, with the same goal of improving statistical accuracy. With a pure empty spectrum and $\mathrm{N}_{2}$ spectrum thus obtained, a pure ${ }^{3} \mathrm{He}$ spectrum could easily be formed by subtracting the pure empty and $\mathrm{N}_{2}$ spectra from a ${ }^{3} \mathrm{He}$ spectrum in the same kinematics, also corrected by charge and deadtime. The dilution factors were then obtained by taking ratios of them for each bin. The results are shown in Table 3.2 for $\mathrm{Q}^{2}=0.1(\mathrm{GeV} / \mathrm{c})^{2}$ and Table 3.3 for $\mathrm{Q}^{2}=0.2(\mathrm{GeV} / \mathrm{c})^{2}$. The statistical errors were negligible.

\begin{tabular}{|c|c|c|}
\hline \hline Ex. $(\mathrm{MeV})$ & $\mathrm{R}^{e m p}(\%)$ & $\mathrm{R}^{N_{2}}(\%)$ \\
\hline 0.00 & 0.27 & 0.76 \\
8.00 & 2.77 & 6.32 \\
13.00 & 2.63 & 5.62 \\
18.00 & 2.37 & 4.83 \\
23.00 & 2.08 & 4.17 \\
28.00 & 1.94 & 3.73 \\
33.00 & 1.86 & 3.44 \\
\hline \hline
\end{tabular}

Table 3.2: Empty and $\mathrm{N}_{2}$ dilution factors at $\mathrm{Q}^{2}=0.1(\mathrm{GeV} / \mathrm{c})^{2}$

\subsubsection{Empty Target and $\mathrm{N}_{2}$ False Asymmetry}

Though specific measures were taken during the experiment to minimize the effect of false asymmetry, it is still a good idea to check whether the empty and $\mathrm{N}_{2}$ false asymmetries are indeed consistent with zero. For each run, the raw asymmetry was calculated in the same way as described in Section 3.2 for ${ }^{3} \mathrm{He}$ raw asymmetry. Before averaging the asymmetry for each run, it had to be corrected for the beam wave plate 


\begin{tabular}{|c|c|c|}
\hline \hline Ex. (MeV) & $\mathrm{R}^{e m p}(\%)$ & $\mathrm{R}^{N_{2}}(\%)$ \\
\hline 0.00 & 0.31 & 2.73 \\
8.00 & 1.36 & 13.12 \\
13.00 & 1.10 & 11.12 \\
18.00 & 0.95 & 9.47 \\
23.00 & 0.83 & 8.19 \\
28.00 & 0.71 & 7.16 \\
33.00 & 0.63 & 6.30 \\
38.00 & 0.55 & 5.49 \\
43.00 & 0.50 & 4.62 \\
48.00 & 0.50 & 3.86 \\
\hline \hline
\end{tabular}

Table 3.3: Empty and $\mathrm{N}_{2}$ dilution factors at $\mathrm{Q}^{2}=0.2(\mathrm{GeV} / \mathrm{c})^{2}$

status for that specific run. We chose to multiply the asymmetry by -1 if the beam wave plate was out. The corrected asymmetry was then averaged over all empty or $\mathrm{N}_{2}$ runs to improve statistical accuracy. The results and their statistical errors are shown in Table 3.4 for $\mathrm{Q}^{2}=0.1(\mathrm{GeV} / \mathrm{c})^{2}$ and Table 3.5 for $\mathrm{Q}^{2}=0.2(\mathrm{GeV} / \mathrm{c})^{2}$.

\begin{tabular}{|c|r|r|}
\hline \hline Ex. $(\mathrm{MeV})$ & \multicolumn{1}{|c|}{$\mathrm{A}^{e m p}(\%)$} & \multicolumn{1}{c|}{$\mathrm{A}^{N_{2}}(\%)$} \\
\hline 0.00 & $-1.51 \pm 0.86$ & $0.05 \pm 0.10$ \\
8.00 & $0.00 \pm 0.47$ & $0.01 \pm 0.06$ \\
13.00 & $0.61 \pm 0.45$ & $-0.01 \pm 0.06$ \\
18.00 & $0.04 \pm 0.44$ & $0.02 \pm 0.06$ \\
23.00 & $1.82 \pm 0.43$ & $-0.05 \pm 0.06$ \\
28.00 & $0.75 \pm 0.41$ & $-0.01 \pm 0.06$ \\
33.00 & $0.26 \pm 0.44$ & $0.11 \pm 0.06$ \\
\hline \hline
\end{tabular}

Table 3.4: Empty and $\mathrm{N}_{2}$ false asymmetries at $\mathrm{Q}^{2}=0.1(\mathrm{GeV} / \mathrm{c})^{2}$

Indeed, except for a couple of outliers, the empty and $\mathrm{N}_{2}$ false asymmetries are consistent with zero within statistical errors. 


\begin{tabular}{|c|r|r|}
\hline \hline Ex. (MeV) & \multicolumn{1}{|c|}{$\mathrm{A}^{e m p}(\%)$} & \multicolumn{1}{|c|}{$\mathrm{A}^{N_{2}}(\%)$} \\
\hline 0.00 & $-0.54 \pm 1.45$ & $-0.29 \pm 0.17$ \\
8.00 & $-0.75 \pm 0.81$ & $-0.07 \pm 0.09$ \\
13.00 & $0.61 \pm 0.76$ & $0.06 \pm 0.08$ \\
18.00 & $-1.37 \pm 0.70$ & $0.11 \pm 0.08$ \\
23.00 & $-0.96 \pm 0.65$ & $0.07 \pm 0.07$ \\
28.00 & $2.39 \pm 0.60$ & $0.09 \pm 0.07$ \\
33.00 & $0.27 \pm 0.57$ & $0.00 \pm 0.06$ \\
38.00 & $-0.99 \pm 0.56$ & $-0.09 \pm 0.06$ \\
43.00 & $0.52 \pm 0.57$ & $0.11 \pm 0.07$ \\
48.00 & $0.51 \pm 0.71$ & $0.06 \pm 0.09$ \\
\hline \hline
\end{tabular}

Table 3.5: Empty and $\mathrm{N}_{2}$ false asymmetries at $\mathrm{Q}^{2}=0.2(\mathrm{GeV} / \mathrm{c})^{2}$

\subsection{Monte Carlo Simulation}

Monte Carlo simulation has become an indispensable tool for electron scattering experiments, primarily for the purposes of acceptance study and radiative correction, both of which are difficult issues to solve in order to extract meaningful physics signals from raw experimental data. In this analysis a Monte Carlo simulation program was built to specificly solve the following problems:

- Simulate the elastic asymmetry with known ${ }^{3} \mathrm{He}$ elastic form factors for each kinematics, and take the ratio of the simulated elastic asymmetry to the experimental elastic asymmetry to determine the product of beam and target polarizations.

- Simulate the elastic radiative tail under the quasielastic spectrum, get the elastic tail dilution factor and asymmetry.

- Calculate the radiative correction to quasielastic asymmetry.

- Study the bin-averaging and acceptance effects. 
Finally, although it is not necessary to have an accurate understanding of the spectrometer acceptance for an asymmetry measurement, a Monte Carlo program can be used to study the systematic uncertainties due to the spectrometer acceptance.

\subsubsection{Overview}

A C ++ simulation program, Polarized Radiative Correction (PRC), was specificly designed for this experiment to exactly calculate the radiative correction in elastic and quasielastic scattering of polarized electrons from polarized targets. In Appendix A there will be a detailed discussion of this program and here we will only give some general introduction.

Following a popular Hall A simulation program, MCEEP [61], PRC employs a uniform random sampling method to populate the experimental acceptance. This method differs from the traditional method of generating events according to a predetermined weighting function, usually the cross section, which more closely resembles the actual experiment. The major advantage of the random sampling method is that it allows us to obtain precise statistics for processes with small cross sections in the presence of a dominant contribution. An event is defined as a set of variables which completely specific the reaction in the laboratory. For each event, some weight variables such as the cross section and asymmetry are calculated according to user-selected physics models. In our simulation, the cross section for elastic scattering from ${ }^{3} \mathrm{He}$ or ${ }^{4} \mathrm{He}$ can be directly calculated from elastic form factor parameterizations, which are fits to previous experimental data. In the case of quasielastic scattering from ${ }^{3} \mathrm{He}$, the ${ }^{3} \mathrm{He}$ response functions have to be calculated using PWIA or Full Faddeev calculations. This is a very slow process and to save computer time, we provide a two-dimensional look-up table for each response function, which is typically a grid of $30 \times 30$ to $100 \times 100$ in the $\left(\omega, Q^{2}\right)$ space, covering the entire experimental acceptance. A two-dimensional spline interpolation is then utilized to obtain the response functions at any $\left(\omega, Q^{2}\right)$ point. The incident electrons traverse through some mate- 
rials before the scattering takes place, and the scattered electrons also pass through some materials before reaching the focal plane. Thus the program has to handle the external radiative correction both before and after the scattering, as well as internal radiative correction which takes place during the primary interaction. The spectrometer model specifies how the scattered electrons are transported to each magnet inside the spectrometer, deflected by the dipole and focused by the quadruples before they reach the focal plane. For each magnet entrance and exit plane, there is an aperture defined by the magnetic field, which defines the spectrometer acceptance.

As can be seen, a simulation process is very complicated and care must be taken to ensure each step comes out right. In the following sections we will discuss each of these issues in more details.

\subsubsection{External Radiative Correction}

When a charged particle traverses through a medium, it might experience small angle deflections due to Coulomb scattering from atomic nuclei in the material. This is called multiple scattering. In addition, the particle might also suffer from ionization energy loss via collisions with atomic electrons. Finally, real photons can be emitted from the interaction between the charged particle and the electromagnetic field of the nuclei in the traversed medium. This process is called external bremsstrahlung.

\section{Multiple Scattering}

The distribution of the deflection angle due to Coulomb interaction is described by the theory of Molière [62]. For small angles, it is roughly a Gaussian with a width given by [63]:

$$
\theta_{0}=\frac{13.6 \mathrm{MeV}}{\beta c p} z \sqrt{x / X_{0}}\left[1+0.038 \ln \left(x / X_{0}\right)\right]
$$

where $p, \beta c$ and $z$ are the momentum, velocity and charge number of the incident particle, and $x / X_{0}$ is the thickness of the scattering medium in radiation lengths.

In the simulation program, a polar scattering angle $\theta_{p}$ is first generated according 
to a Gaussian distribution with width $\theta_{0}$. The final direction of the particle is then randomized over an azimuthal angle of $2 \pi$ around the initial direction of $(\theta, \phi)$, and the deflection angles are sampled by

$$
\begin{aligned}
\Delta \theta & =\theta_{p} \times \cos (2 \pi R) \\
\Delta \phi & =\theta_{p} \times \sin (2 \pi R)
\end{aligned}
$$

where $\mathrm{R}$ is a random number within $[0,1]$.

\section{Most Probable Energy Loss and Energy Straggling}

The ionization energy loss results from the collision between a charged particle and atomic electrons in the medium. The allowed maximum energy transfer from the incident particle to the atomic electrons is

$$
E_{\text {max }}=\frac{2 m_{e} c^{2} \eta^{2}}{1+2 s \sqrt{1+\eta^{2}}+s^{2}}
$$

where $\mathrm{s}=m_{e} / M_{i}$, with $\mathrm{m}_{e}$ being the electron mass and $\mathrm{M}_{i}$ the mass of the incident

particle, and $\eta=\beta \gamma$, with $\beta=v / c$ and $\gamma=1 / \sqrt{1-\beta^{2}}$ for the incident particle. When the incident particle is an electron, the above equation reduces to $E_{\max }=$ $E_{0}-m_{e} c^{2}$, with $E_{0}$ being the incoming electron energy, which means the incoming electron can lose almost all of its energy due to ionization.

The most probable energy loss is given by

$$
\Delta E_{m p}=\frac{2 \pi e^{4} t N Z^{2}}{m_{e} c^{2} \beta^{2} \rho}\left[\ln \frac{4 \pi e^{4} t N Z^{2}}{I^{2}\left(1-\beta^{2}\right) \rho}-\beta^{2}+0.198-\delta\right]
$$

where

$\mathrm{N}$ : the number of electrons per $\mathrm{cm}^{3}$ in the material;

$\rho$ : the density of the material in $\mathrm{g} / \mathrm{cm}^{3}$;

I: the mean excitation potential of the material in $\mathrm{eV}$;

Z: the charge number of the incident particle; 
$\mathrm{t}$ : the path length in the material in $\mathrm{g} / \mathrm{cm}^{2}$;

$\delta$ : the correction for the density effect.

In general, the ionization energy loss of any given particle will not be equal to the most probable energy loss because of statistical fluctuations in the number of collisions and in the energy lost in each collision. The distribution of the energy loss, also called energy straggling, is determined by a parameter $\kappa$, which is the ratio between the most probable energy loss and the maximum energy transfer possible in a single collision, as follows:

$$
\begin{cases}\kappa \leq 0.01 & \text { Landau distribution } \\ 0.01<\kappa \leq 10.0 & \text { Vavilov distribution } \\ 10.0<\kappa & \text { Gaussian distribution }\end{cases}
$$

In this experiment, the most probably energy loss is a few $\mathrm{MeV}$, while the maximum energy transfer possible, which is close to the incoming electron energy, is on the order of $1 \mathrm{GeV}$, thus the energy straggling effect is best described by a Landau distribution. Define

$$
\xi=\frac{2 \pi e^{4} t N Z^{2}}{m_{e} c^{2} \beta^{2}}
$$

then the energy loss of an electron, $\Delta E$, can be transformed to a dimensionless variable $\lambda$ as

$$
\lambda=\frac{\Delta E-\Delta E_{m p}}{\xi}
$$

which follows the Landau distribution with the probability density function given by

$$
\phi(\lambda)=\frac{1}{2 \pi i} \int_{\sigma-i \infty}^{\sigma+i \infty} e^{u \ln u+\lambda u} d u
$$

The shape of $\phi(\lambda)$ is shown in Figure 3-5 together with the integrated probability

$$
\psi(\lambda)=\int_{-\infty}^{\lambda} \phi\left(\lambda^{\prime}\right) d \lambda^{\prime}
$$


In the simulation $\lambda$ is sampled using a Landau random number generator, and the energy loss $\Delta E$ can be determined from Equation (3.12) as

$$
\Delta E=\Delta E_{m p}+\lambda \xi
$$

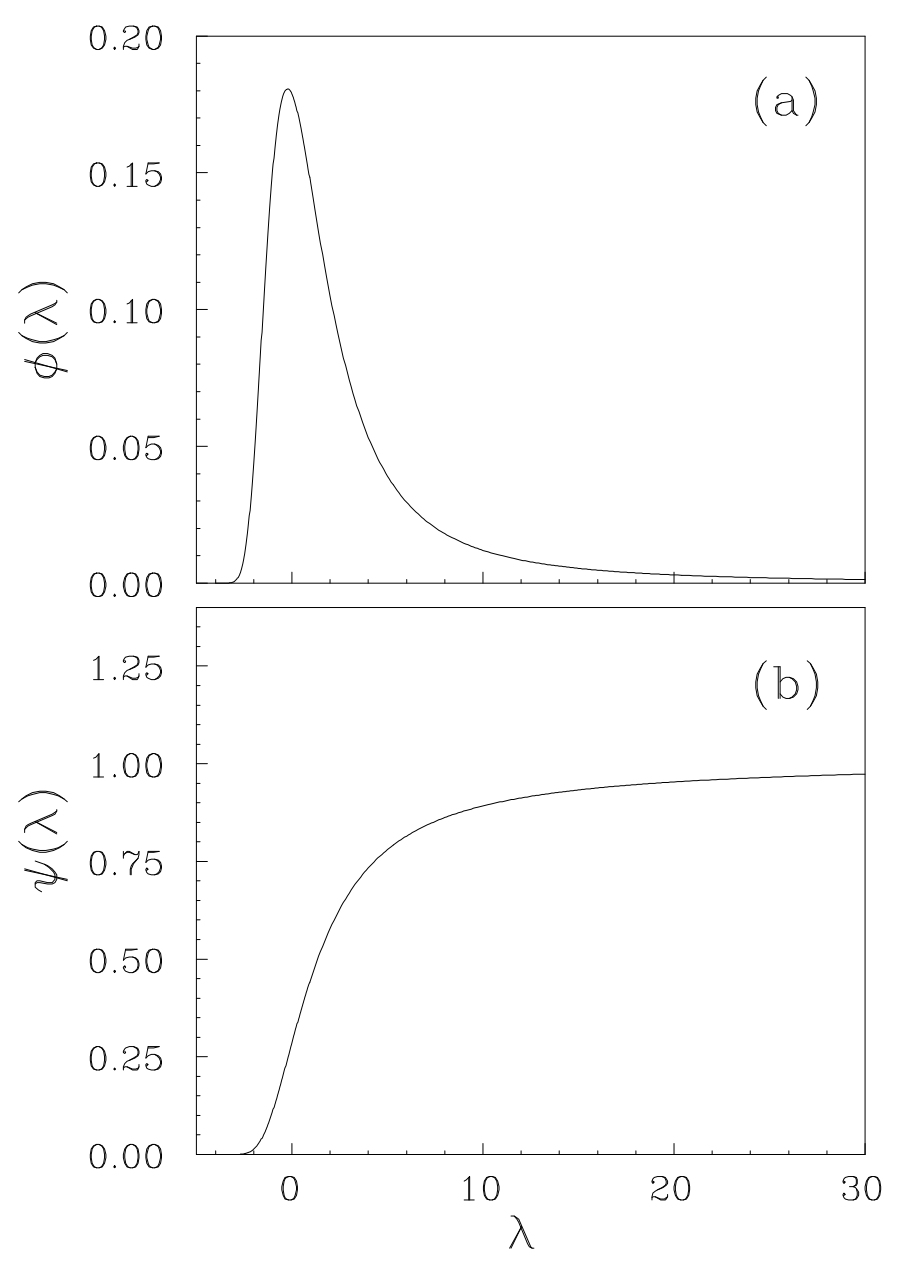

Figure 3-5: The Landau distribution $\phi(\lambda)$ and the integrated probability $\psi(\lambda)$.

\section{External Bremsstrahlung}

Following [64], the probability for a particle with initial energy $E_{0}$ to radiate real 
photons with energy $\Delta E$ when traversing though a material is:

$$
I_{e x t}\left(E_{0}, \Delta E, t\right)=\frac{b t}{\Gamma(1+b t)}\left(\frac{\Delta E}{E_{0}}\right)^{b t}\left\{\frac{1}{\Delta E}\left[1-\frac{\Delta E}{E}+\frac{3}{4}\left(\frac{\Delta E}{E}\right)^{2}\right]\right\}
$$

where $t$ is the material thickness measured in radiation length, and $b$ is the bremsstrahlung parameter given by

$$
b=\frac{4}{3}\left[1+\frac{Z+1}{9(Z+\zeta) \ln \left(183 Z^{-\frac{1}{3}}\right)}\right]
$$

in which $\mathrm{Z}$ is the charge number of target nuclei and $\zeta$ is defined as

$$
\zeta=\frac{\ln \left(1440 Z^{-\frac{2}{3}}\right)}{\ln \left(183 Z^{-\frac{1}{3}}\right)}
$$

To generate the $\Delta E$ distribution according to Eq. (3.16), we adopt the accept-reject technique from [65]. We first define an envelop function that is always bigger than $I_{\text {ext }}\left(E_{0}, \Delta E, t\right)$ as

$$
\mathcal{E}(\Delta E)=1.1 \frac{b t}{\Gamma(1+b t) E_{0}^{b t}} \Delta E^{b t-1}
$$

Since the envelop function can be integrated analytically, it is straightforward to generate a trial value of $\Delta E$ that follows the distribution given by the envelop function. This can be seen by looking at the integral and partial integral of the envelop function:

$$
\begin{aligned}
\int_{0}^{E_{0}} \mathcal{E}(x) d x & =\frac{1.1}{\Gamma(1+b t)} \\
\int_{0}^{\Delta E} \mathcal{E}(x) d x & =\frac{1.1}{\Gamma(1+b t)} \frac{\Delta E^{b t}}{E_{0}^{b t}}
\end{aligned}
$$

Thus to create a $\Delta E$ distribution with $\mathcal{E}(\Delta E)$ as its shape, we simply generate a uniform random number $r_{1}$ on $\left[0, \frac{1.1}{\Gamma(1+b t)}\right]$ and set

$$
r_{1}=\frac{1.1}{\Gamma(1+b t)} \frac{\Delta E_{t r i a l}^{b t}}{E_{0}^{b t}}
$$


or

$$
\Delta E_{\text {trial }}=E_{0}\left(\frac{\Gamma(1+b t)}{1.1} r_{1}\right)^{\frac{1}{b t}}
$$

Equivalently we can also generate a random number $r_{1}$ on $[0,1]$, then simply set

$$
\Delta E_{\text {trial }}=E_{0} r_{1}^{\frac{1}{b t}}
$$

We then use the accept-reject technique to determine whether this candidate value $\Delta E_{\text {trial }}$ is accepted or not. Generate another random number $r_{2}$ on $[0,1]$, if

$$
r_{2} \mathcal{E}\left(\Delta E_{\text {trial }}\right)>I_{\text {ext }}\left(E_{0}, \Delta E_{\text {trial }}, t\right)
$$

then the generated value does not fall within the true bremsstrahlung distribution, and the process is restarted with new values for $r_{1}$ and $r_{2}$, otherwise $\Delta E_{\text {trial }}$ is accepted as the true bremsstrahlung energy loss for this event.

\section{Materials Before and After Scattering}

To apply external radiative corrections to each event in the simulation, we need to know the property of each material the electron passes through, both before and after the scattering process. A schematic drawing of the materials is shown in Figure 3-6 and their properties are shown in Table 3.6.

\subsubsection{Internal Radiative Correction}

Consider a typical $\left(\mathrm{e}, \mathrm{e}^{\prime}\right)$ reaction. Most theoretical work is only concerned with the first-order Born approximation, which is the one-photon exchange Feynman diagram shown in Chapter 1. However, in reality there are also higher order Feynman diagrams contributing to this process, and it is up to the experimentalist to make the so-called "radiative corrections" on the data before any meaningful comparison between the theory and the experiment can be made. In our analysis we only need to consider the second order corrections, because at our energy the higher order con- 


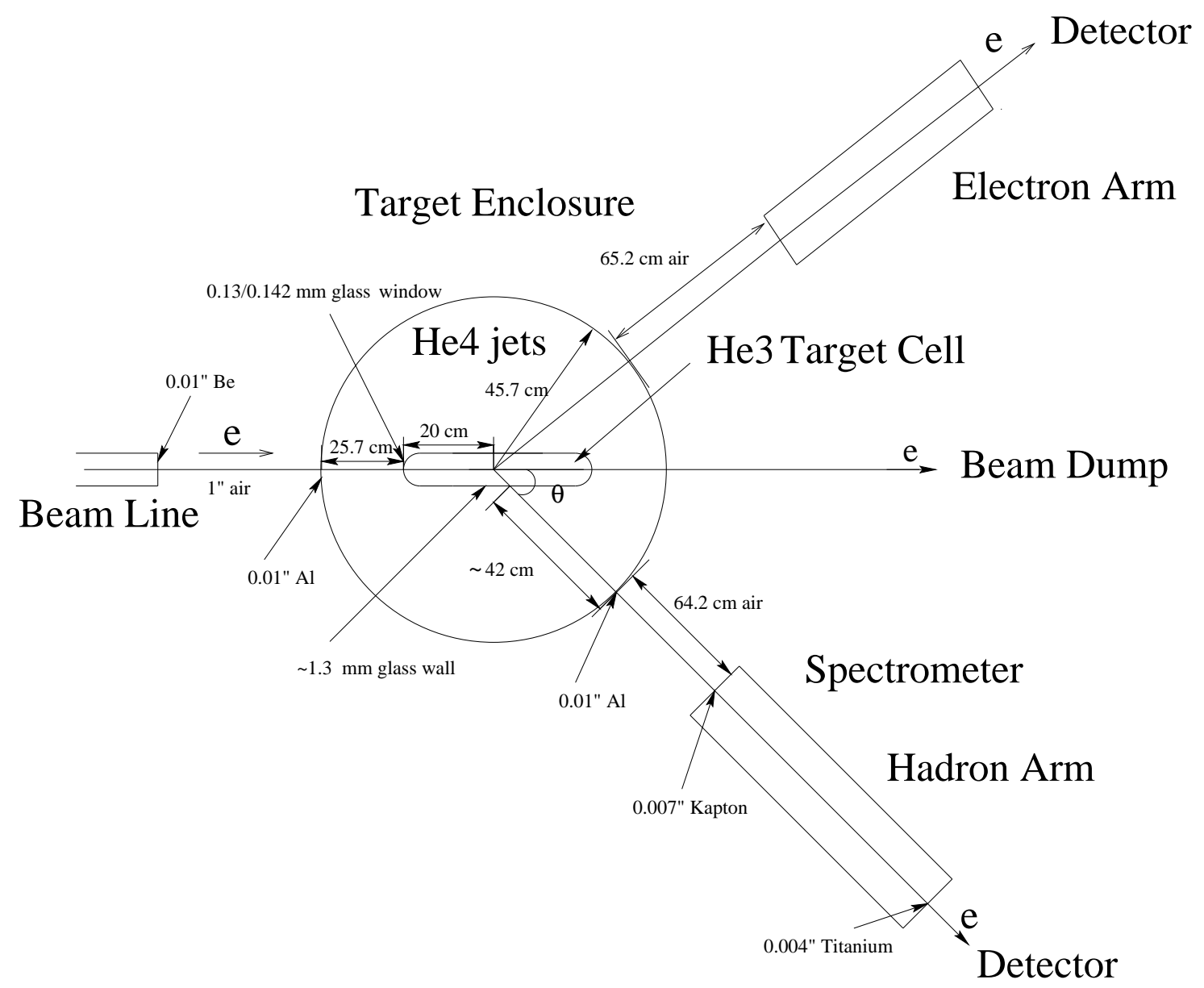

Figure 3-6: Schematic drawing of radiation materials before and after scattering

tributions are completely negligible. Also since the target mass is much larger than the electron mass, we only need to concern ourselves with the radiation on the electron side of the diagram. The six relevant Feynman diagrams for radiative correction are shown in Figure 3-7. Diagrams a and b involve real photons radiation and are called internal bremsstrahlung. Diagrams $\mathrm{c}$ and $\mathrm{d}$ correspond to the emission and re-absorption of a virtual photon by the incident or scattering electron, which results in the renormalization of the electron mass. Diagram e shows emission of a virtual photon by the incident electron and then the reaborption of the same photon by the scattered electron, and gives rise to the vertex renormalization. Diagram $\mathrm{f}$ is called vacuum polarization and involves the creation of a virtual electron-positron pair from 


\begin{tabular}{|c|c|c|c|c|c|}
\hline \hline material & $\mathrm{Z}$ & $\mathrm{Z} / \mathrm{A}$ & $\begin{array}{c}\mathrm{X}_{0} \\
\left(\mathrm{~g} / \mathrm{cm}^{2}\right)\end{array}$ & $\begin{array}{c}\text { length } \\
(\mathrm{cm})\end{array}$ & $\begin{array}{c}\text { density } \\
\left(\mathrm{g} / \mathrm{cm}^{3}\right)\end{array}$ \\
\hline beryllium & 4 & 0.44384 & 65.19 & 0.0254 & 1.848 \\
air & 14.5 & 0.49919 & 36.66 & 2.54 & $1.205 \times 10^{-3}$ \\
aluminum & 13 & 0.48181 & 24.01 & 0.0254 & 2.70 \\
${ }^{4} \mathrm{He}$ & 2 & 0.49968 & 94.32 & 25.7 & $1.664 \times 10^{-4}$ \\
$\mathrm{SiO}_{2}$ & 30 & 0.49707 & 28.30 & 0.013 & 2.23 \\
${ }^{3} \mathrm{He}$ & 2 & 0.66667 & 70.74 & 20.0 & $1.544 \times 10^{-3}$ \\
$\mathrm{SiO}$ & 30 & 0.49707 & 28.30 & 0.13 & 2.23 \\
${ }^{4} \mathrm{He}$ & 2 & 0.49968 & 94.32 & 25.7 & $1.664 \times 10^{-4}$ \\
aluminum & 13 & 0.48181 & 24.01 & 0.0254 & 2.70 \\
air & 14.5 & 0.49919 & 36.66 & 64.2 & $1.205 \times 10^{-3}$ \\
Kapton & 2.7 & 0.51264 & 40.56 & 0.0178 & 1.42 \\
Titanium & 22 & 0.45948 & 16.17 & 0.0102 & 4.54 \\
\hline \hline
\end{tabular}

Table 3.6: Properties of radiation materials before and after scattering

the exchanged virtual photon.

Currently there are two methods to calculate the internal radiative correction. In the first method, real photons are classified according to whether their energies are below a certain cutoff energy (soft photons) or above the cutoff (hard photons). Usually the cutoff energy is chosen to be the experimental energy resolution. The virtual photon and soft photon contributions together are referred to as the Schwinger correction [66], which is just a multiplication factor dependent on the photon cutoff energy and some kinematics factors, and thus has the remarkable property of being spin-independent. The hard photon emission leads to the radiative tail, which can be calculated using the peaking approximation method proposed by Mo and Tsai [67], but only for the case of unpolarized scattering.

The second method [68], originally proposed by Bardin and Shumeiko in [69], and developed in [70-72], uses a covariant formalism to exactly calculate the radiative correction for deep inelastic scattering of polarized lepton scattering from polarized nuclei. This method can be easily generated to the case of quasielastic scattering, 


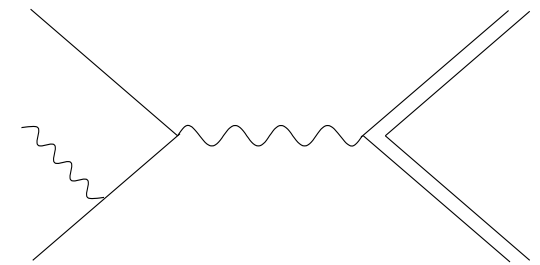

a

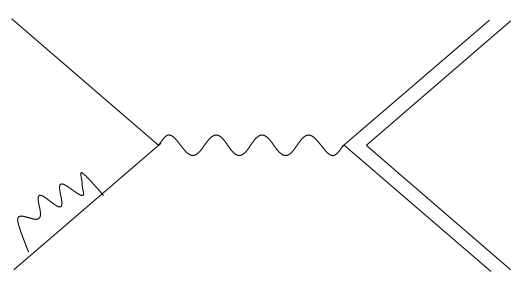

$\mathrm{c}$

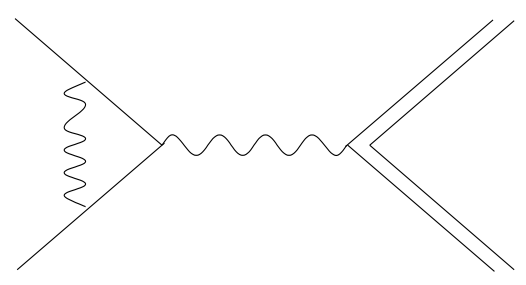

$\mathrm{e}$

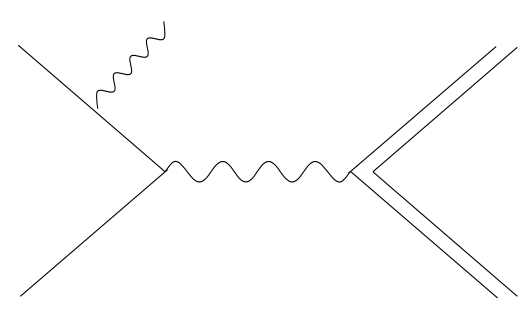

$\mathrm{b}$

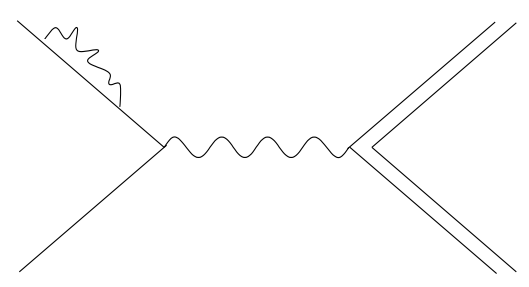

d

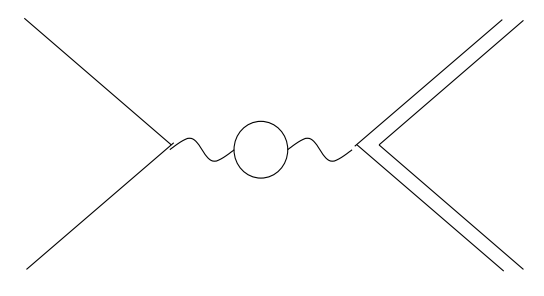

$\mathrm{f}$

Figure 3-7: Second order Feynman diagrams for internal radiative correction

and can be used to calculate the spin-dependent elastic radiative tail and quasielastic radiative tail in our case.

In the simulation, we separate the elastic spectrum into two parts. The first part is the elastic peak, where the kinematics is calculated assuming no radiation. The second part is the elastic radiative tail with the radiated photon energy larger than the soft photon cutoff. We will apply the Schwinger correction with the soft photon cutoff to the elastic peak, while using the covariant method to calculate the elastic radiative tail and the quasielastic cross section with radiative correction. 


\subsubsection{Schwinger Correction}

There are many versions for the Schwinger correction factor, and the one we will use is taken from Penner [73] and has the following form

$$
C_{s c h w}=e^{-\delta_{r}}\left(1-\delta_{v}\right)
$$

where $\delta_{r}$ is the real soft photon contribution and $\delta_{v}$ is the virtual photon contribution. There factors are given by

$$
\begin{aligned}
& \delta_{r}=\frac{\alpha}{\pi}\left(\ln \frac{Q^{2}}{m_{e}^{2}}-1\right) \ln \left[\frac{E^{2}}{\xi^{3}} \frac{1}{\Delta^{2}}\right] \\
& \delta_{v}=\frac{\alpha}{\pi}\left[\frac{28}{9}-\frac{13}{6} \ln \frac{Q^{2}}{m_{e}^{2}}+\frac{\pi^{2}}{6}-\phi\left(\cos ^{2} \frac{\theta_{e}}{2}\right)\right]
\end{aligned}
$$

for elastic scattering, where

$$
\xi=1+\frac{E}{M_{T}}\left(1-\cos \theta_{e}\right)
$$

and $\phi(x)$ is the Spence function defined by

$$
\phi(x)=-\int_{0}^{x} \frac{\ln (1-y)}{y} d y
$$

In the above, $\Delta$ is the soft photon cutoff energy.

During the simulation $C_{s c h w}$ is applied to the elastic cross section as a multiplication factor, and the kinematics factors are all evaluated at the elastic peak, which is reasonable since the photon cutoff energy is chosen to be around the experimental energy resolution.

\subsubsection{Covariant Methods for Polarized Radiative Correction}

As previously mentioned, the covariant method for calculating radiative correction was originally developed for deep inelastic scattering process, and the formula used 
there is quite different from the one we described in Chapter 1. Thus we will first introduce the new Born cross section formula from [68] and compare it with the original formula, then write down the results for radiative corrected cross section, also from [68].

\section{Born Cross Section}

The Born cross section for inclusive scattering of polarized electrons from polarized spin- $\frac{1}{2}$ nuclei, such as proton and ${ }^{3} \mathrm{He}$, can be written as

$$
\begin{aligned}
\frac{d^{2} \sigma}{d x d y}=\frac{4 \pi \alpha^{2}}{\lambda_{s}} \frac{S S_{x}}{Q^{4}}\{ & \left(Q^{2}-2 m^{2}\right) J_{1}+\left(S X-M^{2} Q^{2}\right) \frac{J_{2}}{2 M^{2}} \\
& +h P_{L} m M\left[2\left(Q^{2} \xi \cdot \eta-q \eta k_{2} \cdot \xi\right) \frac{J_{3}}{M^{2}}\right. \\
& \left.\left.+\left(S_{x} k_{2} \cdot \xi-2 \xi \cdot p Q^{2}\right) q \cdot \eta \frac{J_{4}}{M^{4}}\right]\right\}
\end{aligned}
$$

where $m$ and $M$ are the masses of the electron and the target, $k_{1}, k_{2}, p$ are the fourmomenta of the incident electron, the scattered electron and the initial target, $q$ is the four-momentum transfer and $Q^{2}$ is the minus four-momentum transfer squared, $Q^{2}=-q^{2}, h$ is the electron helicity while $P_{L}$ is the beam polarization. The other kinematics variables are defined as

$$
\begin{aligned}
S & =2 p \cdot k_{1} \\
S_{x} & =2 p \cdot q \\
X & =2 p \cdot k_{2} \\
x & =\frac{Q^{2}}{2 p \cdot q} \\
y & =\frac{p \cdot q}{p \cdot k_{1}}
\end{aligned}
$$

and $\xi$ and $\eta$ are the electron and target polarization four-vectors. Finally $J_{1}, J_{2}, J_{3}$ and $J_{4}$ are four dimensionless structure functions for the target nucleus.

After some algebraic manipulation, the above equation can be transformed into a 
more familiar formula

$$
\begin{aligned}
\frac{d^{2} \sigma}{d E^{\prime} d \Omega}=\frac{\alpha^{2} \cos ^{2} \frac{\theta}{2}}{4 E^{2} M \sin ^{4} \frac{\theta}{2}} & \left\{J_{2}+2 \tan ^{2} \frac{\theta}{2} J_{1}\right. \\
+ & 2 h P_{L} \tan ^{2} \frac{\theta}{2}\left[-\left(E \cos \theta_{p}+E^{\prime} \cos \left(\theta-\theta_{p}\right)\right) \frac{J_{3}}{M}\right. \\
& \left.\left.-\left(E+E^{\prime}\right)\left(E^{\prime} \cos \left(\theta-\theta_{p}\right)-E \cos \theta_{p}\right) \frac{J_{4}}{M}\right]\right\}
\end{aligned}
$$

Compare Equation (3.32) with Equation (1.31) in Chapter 1, we find

$$
\left\{\begin{aligned}
J_{1} & =M W_{1} \\
J_{2} & =M W_{2} \\
J_{3} & =-M P_{t}\left(G_{1}+\frac{\omega}{M} G_{2}\right) \\
J_{4} & =-M P_{t} G_{2}
\end{aligned}\right.
$$

Thus the four new structure functions can be calculated from the structure functions we introduced in Chapter 1.

For quasielastic scattering, the structure functions can be expressed as a combination of response functions as follows:

$$
\begin{aligned}
W_{1} & =\frac{1}{2} R_{T} \\
W_{2} & =\frac{Q^{4}}{|\vec{q}|^{4}} R_{L}+\frac{1}{2} \frac{Q^{2}}{|\vec{q}|^{2}} R_{T} \\
G_{1} & =\frac{1}{2} \frac{M^{2}}{|\vec{q}|^{2}}\left[-\frac{Q^{2}}{\sqrt{2} M|\vec{q}|} R_{T L^{\prime}}+\frac{\omega}{M} R_{T^{\prime}}\right] \\
G_{2} & =-\frac{1}{2} \frac{M^{2}}{|\vec{q}|^{2}}\left[-\frac{\omega}{\sqrt{2}|\vec{q}|} R_{T L^{\prime}}+R_{T^{\prime}}\right]
\end{aligned}
$$

where $\vec{q}$ is the three-momentum transfer, and

$$
|\vec{q}|^{2}=\omega^{2}+Q^{2}
$$

For elastic scattering, the structure functions can be calculated from electric and 
magnetic form factors as

$$
\begin{aligned}
W_{1} & =\tau G_{M}^{2} \delta\left(1-\frac{Q^{2}}{2 M \omega}\right) \\
W_{2} & =\frac{G_{E}^{2}+\tau G_{M}^{2}}{1+\tau} \delta\left(1-\frac{Q^{2}}{2 M \omega}\right) \\
G_{1} & =-\frac{G_{M}}{2} \frac{G_{E}+\tau G_{M}}{1+\tau} \delta\left(1-\frac{Q^{2}}{2 M \omega}\right) \\
G_{2} & =\frac{G_{M}}{4} \frac{G_{M}-G_{E}}{1+\tau} \delta\left(1-\frac{Q^{2}}{2 M \omega}\right)
\end{aligned}
$$

where $\tau=\frac{Q^{2}}{4 M^{2}}$. Thus for elastic scattering, we have

$$
\begin{aligned}
J_{i}^{e l} & =\tilde{J}_{i}^{e l} \delta\left(1-\frac{Q^{2}}{2 M \omega}\right) \\
\tilde{J}_{1}^{e l} & =M \tau G_{M}^{2} \\
\tilde{J}_{2}^{e l} & =M \frac{G_{E}^{2}+\tau G_{M}^{2}}{1+\tau} \\
\tilde{J}_{3}^{e l} & =M P_{t} \frac{G_{M} G_{E}}{2} \\
\tilde{J}_{4}^{e l} & =M P_{t} \frac{G_{M}\left(G_{E}-G_{M}\right)}{4(1+\tau)}
\end{aligned}
$$

\section{Radiative Cross section}

Following the covariant method, the cross section with second order radiative corrections can be written explicitly as follows

$$
\sigma_{r}=\left[1+\frac{\alpha}{\pi}\left(\delta_{v e r t}+\delta_{v a c}+\delta_{R}^{I R}\right)\right] \sigma_{0}+\sigma_{F}
$$

where

$\sigma_{0}:$ Born cross section

$\delta_{\text {vert }}$ : vertex correction term (divergent)

$\delta_{v a c}$ : vacuum polarization term (finite)

$\delta_{R}^{I R}$ : infrared divergent part extracted from internal bremsstrahlung radiative tail

$\sigma_{F}$ : internal bremsstrahlung radiative tail, with the infrared divergent part extracted 
Considering the multiple photon emission effects and noting that the divergent terms, $\delta_{\text {vert }}$ and $\delta_{R}^{I R}$, cancel each other to leave a finite term, the above radiative cross section becomes [74]

$$
\sigma_{r}=e^{\delta_{i n f}}\left[1+\frac{\alpha}{\pi}\left(\delta_{v r}+\delta_{v a c}\right)\right] \sigma_{0}+\sigma_{F}
$$

where

$$
\begin{aligned}
\delta_{i n f} & =\left(l_{m}-1\right) \ln \frac{\left(W^{2}-M_{c}^{2}\right)^{2}}{\left(X+Q^{2}\right)\left(S-Q^{2}\right)} \\
\delta_{v a c} & =\frac{2}{3}\left(Q^{2}+2 m^{2}\right) L_{m}-\frac{10}{9}+\frac{8 m^{2}}{3 Q^{2}}\left(1-2 m^{2} L_{m}\right) \\
\delta_{v r} & =\frac{3}{2} l_{m}-2-\frac{1}{2} \ln ^{2} \frac{X}{S}-\frac{\pi^{2}}{6}+\phi\left(\frac{S X-Q^{2} M^{2}}{\left(X+Q^{2}\right)\left(S-Q^{2}\right)}\right)
\end{aligned}
$$

with

$$
\begin{aligned}
W^{2} & =M^{2}-Q^{2}+2 M \omega \\
l_{m} & =\ln \frac{Q^{2}}{m^{2}} \\
L_{m} & =\frac{\ln \frac{\sqrt{\lambda_{m}}+Q^{2}}{\sqrt{\lambda_{m}}-Q^{2}}}{\sqrt{\lambda_{m}}} \\
\lambda_{m} & =Q^{4}+4 m^{2} Q^{2}
\end{aligned}
$$

$M_{c}$ is the invariant mass corresponding to the threshold of the reaction channel. For deep inelastic scattering of electrons from protons, $M_{c}$ corresponds to the pion threshold, $M_{c}=M_{p}+M_{\pi}$. For ${ }^{3} \mathrm{He}$ quasielastic scattering, it corresponds to the two-body breakup threshold, $M_{c}=M_{3_{H e}}+E_{b}$, with $E_{b}=5.5 \mathrm{MeV}$.

The major advantage of the covariant method is that it gives an exact, albeit complicated, formula for the last term, the internal bremsstrahlung radiative tail with infrared divergent part subtracted, and the formula is applicable to both unpolarized 
and polarized inclusive scattering

$$
\begin{gathered}
\sigma_{F}=-\frac{\alpha^{3} E^{\prime}}{2 \pi M E} \int_{\tau_{\min }}^{\tau_{\max }} d \tau \sum_{i=1}^{4}\left\{\theta_{i 1}(\tau) \int_{0}^{R_{\max }} \frac{d R}{R}\left[\frac{J_{i}(R, \tau)}{\left(Q^{2}+R \tau\right)^{2}}-\frac{J_{i}(0,0)}{Q^{4}}\right]\right. \\
\left.+\sum_{j=2}^{k_{i}} \theta_{i j}(\tau) \int_{0}^{R_{\max }} d R \frac{R^{j-2}}{\left(Q^{2}+R \tau\right)^{2}} J_{i}(R, \tau)\right\}
\end{gathered}
$$

with $\mathrm{k}$ being the emitted photon four-momentum and

$$
\begin{aligned}
R & =2 p \cdot k \\
R_{\text {min }} & =0 \\
R_{\text {max }} & =\frac{W^{2}-M_{c}^{2}}{1+\tau} \\
\tau & =\frac{k \cdot\left(k_{1}-k_{2}\right)}{p \cdot k} \\
\tau_{\text {min }} & =\frac{S_{x}-\sqrt{\lambda_{Q}}}{2 M^{2}} \\
\tau_{\text {max }} & =\frac{S_{x}+\sqrt{\lambda_{Q}}}{2 M^{2}} \\
\lambda_{Q} & =S_{x}^{2}+4 M^{2} Q^{2}
\end{aligned}
$$

$\theta_{i j}$ 's are some very complicated variables depending only on kinematics factors and please refer to [68] for details on $k_{i}$ and $\theta_{i j}$.

To get the elastic radiative tail, simply plug Eq. (3.35) into Eq. (3.45), and we obtain

$$
\sigma^{e r t}=-\frac{\alpha^{3} E^{\prime}}{2 \pi M E} \int_{\tau_{\min }}^{\tau_{\max }} d \tau \sum_{i=1}^{4} \sum_{j=1}^{k_{i}} \theta_{i j} \frac{2 M R_{e l}^{j-2}}{(1+\tau)\left(Q^{2}+R \tau\right)^{2}} \tilde{J}_{i}^{e l}
$$

with

$$
R_{e l}=\frac{S_{x}-Q^{2}}{1+\tau}
$$

\section{Practical Issues in Simulation}

There are two major issues in applying the above formulas to a simulation program, mainly due to the numerical integration over the integrand. 


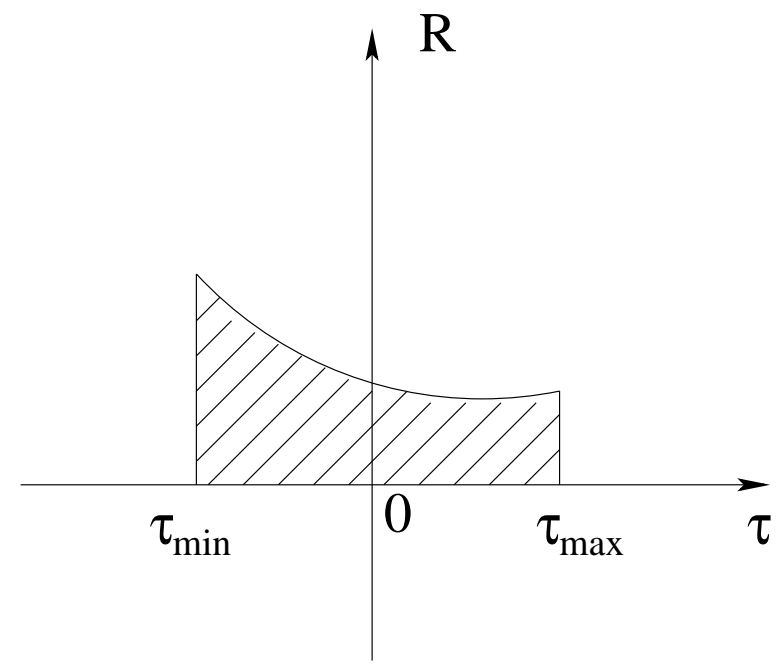

Figure 3-8: Integration region over $R$ and $\tau$ is not a rectangle

The first issue involves the two-dimensional integration over $R$ and $\tau$. The integration region over $R$ and $\tau$ is not a rectangle, as shown in Figure 3-8, so it is not easy to apply numerical methods. To make the numerical methods applicable, we have to transform $\mathrm{R}$ to another dimensionless variable $v$ as

$$
v=\frac{(1+\tau) R}{2 M \omega}
$$

and Eq. (3.45) becomes

$$
\begin{aligned}
\sigma_{F}=-\frac{\alpha^{3} E^{\prime}}{2 \pi M E} \int_{\tau_{\min }}^{\tau_{\max }} & d \tau \sum_{i=1}^{4}\left\{\theta_{i 1}(\tau) \int_{0}^{v_{\max }} \frac{d v}{v}\left[\frac{J_{i}(R, \tau)}{\left(Q^{2}+R \tau\right)^{2}}-\frac{J_{i}(0,0)}{Q^{4}}\right]\right. \\
& \left.+\sum_{j=2}^{k_{i}} \theta_{i j}(\tau) \int_{0}^{v_{\max }} d v \frac{2 M \omega}{1+\tau} \frac{R^{j-2}}{\left(Q^{2}+R \tau\right)^{2}} J_{i}(R, \tau)\right\}
\end{aligned}
$$

with

$$
\left\{\begin{array}{l}
v_{\min }=0 \\
v_{\max }=\frac{W^{2}-M_{c}^{2}}{2 M \omega}
\end{array}\right.
$$

and now the integration region becomes a rectangle, as shown in Figure 3-9. Also in 
numerical integration $v_{\min }$ should be chosen to be some very small number instead of 0.

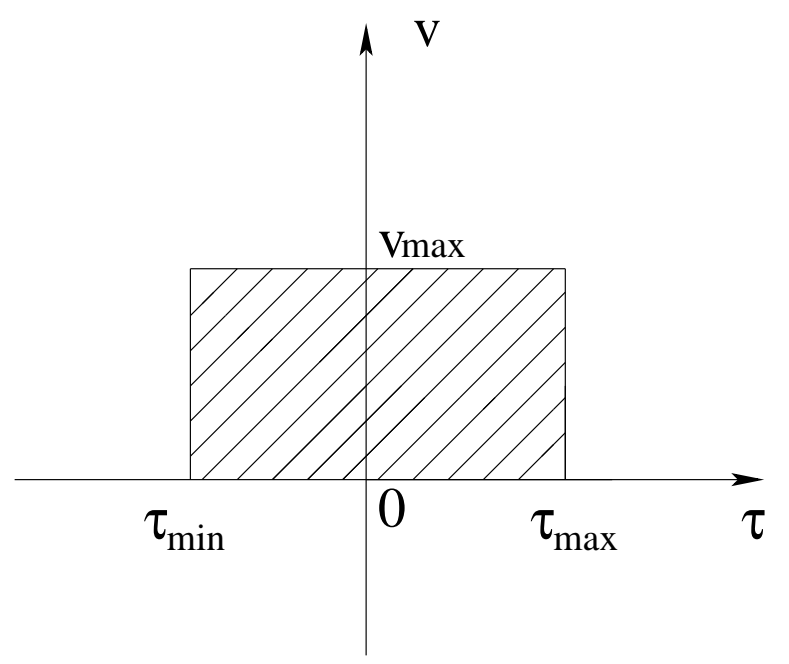

Figure 3-9: Integration region over $v$ and $\tau$ is a rectangle

The second issue concerns the integration over $\tau$. As is well known, the integrand in Eq. (3.45) is very sharply peaked when $\tau$ is equal to $\tau_{s}$ or $\tau_{p}$, namely most of the photons are emitted along the direction of the incident or scattered electron. This feature of the integrand is actually the basis for the peaking approximation method of Mo and Tsai [67]. In our case, it introduces significant difficulty in numerical integration over the integrand, and an unacceptably long computing time is needed to achieve a certain precision. However, a careful study shows that all $\theta_{i j}$ 's, complicated as they appear, can be analytically integrated out, and since the structure functions and all other factors besides $\theta$ in the integrand changes slowly across the two peaks, we can analytically integrate the two peaks out by setting these other factors to their values at each peak, and then use numerically methods to integrate the residual part, which is much smaller compared with the peak and thus the integration converges very rapidly. 


\subsubsection{Spectrometer Model and Acceptance}

A spectrometer model is used to transport charged particles through elements of the spectrometer on an event-by-event basis to see whether they can reach the focal plane. A realistic model is essential for a deep understanding of the spectrometer acceptance, which is of ultimate importance to any cross section measurement. For an asymmetry measurement as our experiment, an exact determination of the acceptance is not necessary. On the other hand, a spectrometer model can be used to understand the systematic errors of physics asymmetry due to the acceptance, as we will see in Chapter 4.

The spectrometer model for Hall A High Resolution Spectrometers is based on SNAKE models. It consists of a set of forward transfer functions, which transport particles from the target region to Q1 exit, dipole entrance and exit, Q3 entrance and exit, and the focal plane, a set of reverse transfer functions, which can be used to reconstruct the target region variables from the focal plane variables, and the geometric aperture of each magnet entrance or exit plane, which defines the nominal spectrometer acceptance: if the particle transported to this magnet plane is outside of the geometric aperture, it is discarded.

The geometric aperture at Q1 exit is a circle of radius $0.1492 \mathrm{~m}$, those at Q3 entrance and exit are circles of radius $0.300 \mathrm{~m}$, while the apertures at the dipole entrance and exit are trapezoids. However, these apertures do not necessarily represent the physics apertures, which depend on both the magnetic field inside each magnet and the particle charge and momentum. This is manifested in Figure 3-10, which compares the electron distribution at the dipole entrance from data and that from a simulation with only geometric aperture cuts.

A careful determination of the spectrometer acceptance by means of physics apertures inside each magnet can only be done for each individual experiment, as was done for JLab Hall A experiment E94-010 [75]. Another popular method to understand the acceptance, called R-function, was proposed after our analysis was finished 


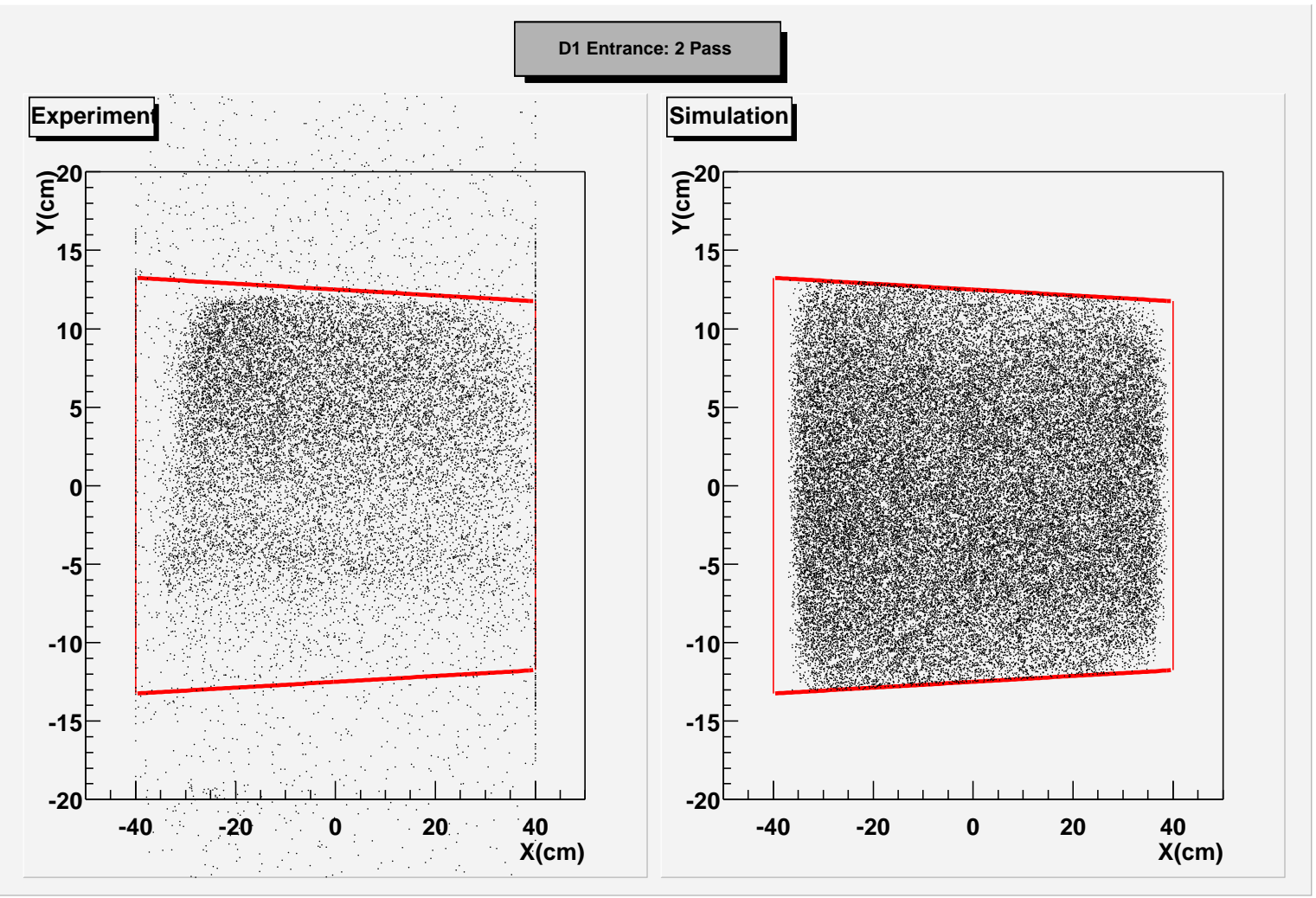

Figure 3-10: The electron distribution at the entrance of dipole from data and that from a simulation with geometric aperture cuts. The trapezoid shown is the geometric aperture for the dipole entrance

and focuses on the target region variable distribution instead of the magnet apertures. However this method usually requires substantial cuts on the data, thereby greatly reduces the statistical accuracy. As previously mentioned, for an asymmetry measurement as our experiment, a precise determination of the spectrometer acceptance is not necessary. A simple yet powerful method we adopted is to look at the electron distribution at the above magnet entrance and exit planes from data, determine a suitable aperture for each plane and apply it to the simulation. In spite of its simplicity, this method turns out to be quite successful in understanding the 
experimental acceptance. The same comparison of the electron distribution at the entrance of dipole from data and that from a simulation with physics aperture cuts is shown in Figure 3-11.

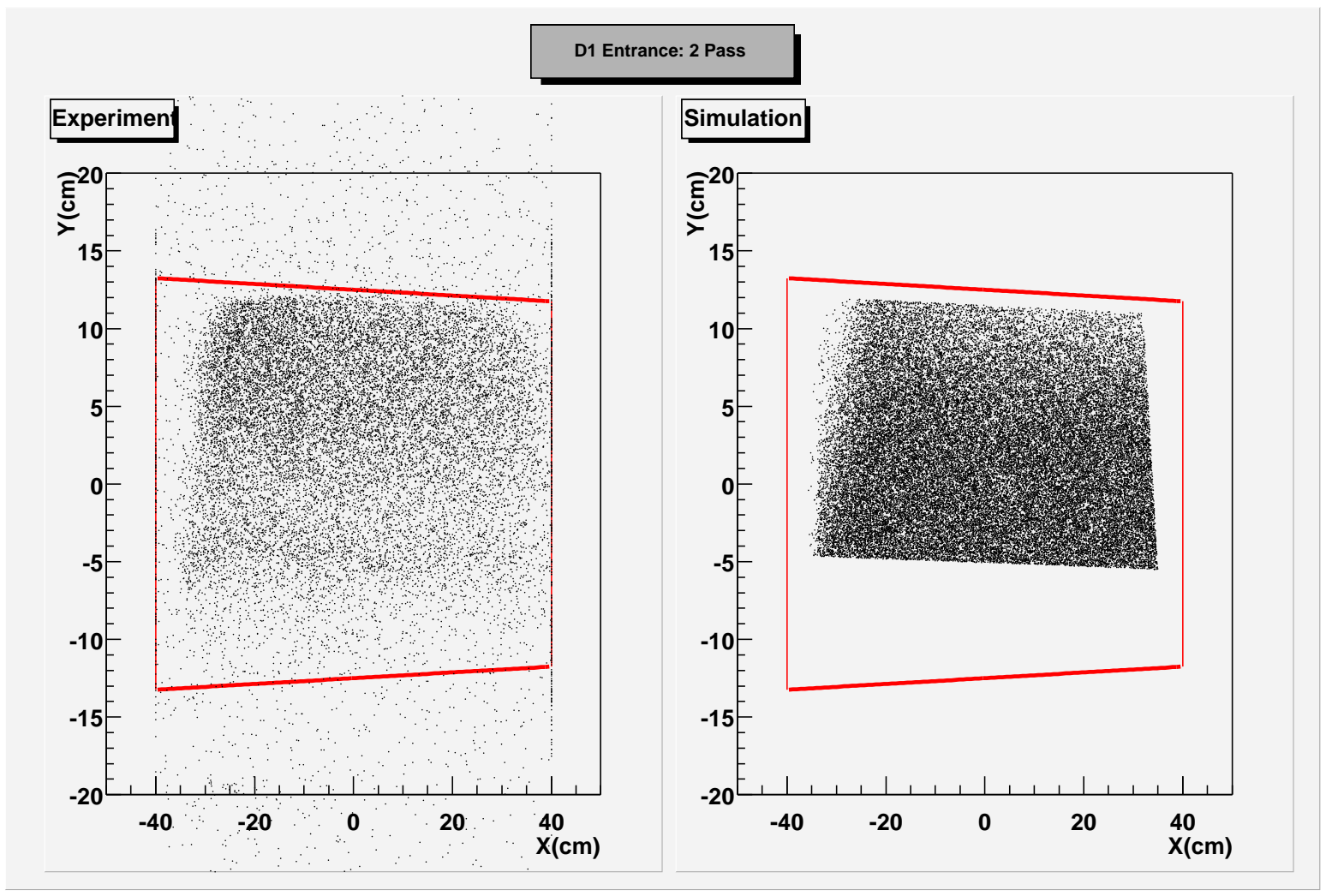

Figure 3-11: The electron distribution at the entrance of dipole from data and that from a simulation with physics aperture cuts

However, it is to be noted that this method is only one step ahead towards a thorough understanding of the spectrometer acceptance, which is a notoriously difficult problem for all Hall A experiments, mainly due to the lack of precise knowledge of the field distribution inside each magnet. New spectrometer models incorporating some R-function cuts are still being designed and tested [76]. 


\subsection{5 ${ }^{4}$ He Radiative Tail Simulation}

To test the radiative correction part of the simulation, some reference ${ }^{4} \mathrm{He}$ runs were taken at each kinematics during the experiment. This is because the two-body breakup energy of the ${ }^{4} \mathrm{He}$ nucleus is $19.81 \mathrm{MeV}$ and thus the elastic scattering of electrons from ${ }^{4} \mathrm{He}$ has a very long radiative tail. The ${ }^{4} \mathrm{He}$ elastic form factors are very well known factors, thus by comparing the shape of the radiative tail from the simulation to that from the data, we can get some idea of how good is the treatment of radiative correction in the simulation. In addition, since the ${ }^{4} \mathrm{He}$ density in the reference cell was measured to a high precision during the experiment, the overall normalization shows how good is our understanding of the spectrometer acceptance.

Such a comparison is shown in Figure 3-12 for $\mathrm{Q}^{2}=0.1(\mathrm{GeV} / \mathrm{c})^{2}$. As can be seen, the agreement for both the elastic peak and the elastic radiative tail is excellent between data and simulation, which gives us confidence in the simulation program.

\subsection{Elastic Polarimetry}

The product of beam and target polarizations, $P_{b} P_{t}$, were monitored continuously during the experiment using the technique of elastic polarimetry. This is based on the fact that the ${ }^{3} \mathrm{He}$ elastic form factors, the charge form factor $F_{c}$ and the magnetic form factor $F_{m}$, are known very well experimentally [77], and the ${ }^{3} \mathrm{He}$ elastic asymmetry can be calculated to a good precision as:

$$
A^{e l}=\frac{-2 \tau v_{T^{\prime}} \cos \theta^{*} \mu_{A}^{2} F_{m}^{2}+2 \sqrt{2 \tau(1+\tau)} v_{T L^{\prime}} \sin \theta^{*} \cos \phi^{*} \mu_{A} Z F_{m} F_{c}}{(1+\tau) v_{L} Z^{2} F_{c}^{2}+2 \tau v_{T} \mu_{A}^{2} F_{m}^{2}}
$$

If we simulate the elastic asymmetry under the same conditions as in the experiment, the ratio between the measured asymmetry and the simulated asymmetry, after correcting for the empty target and $N_{2}$ dilution factors, should give us the product of beam and target polarizations. The comparison between data and simulation for both 


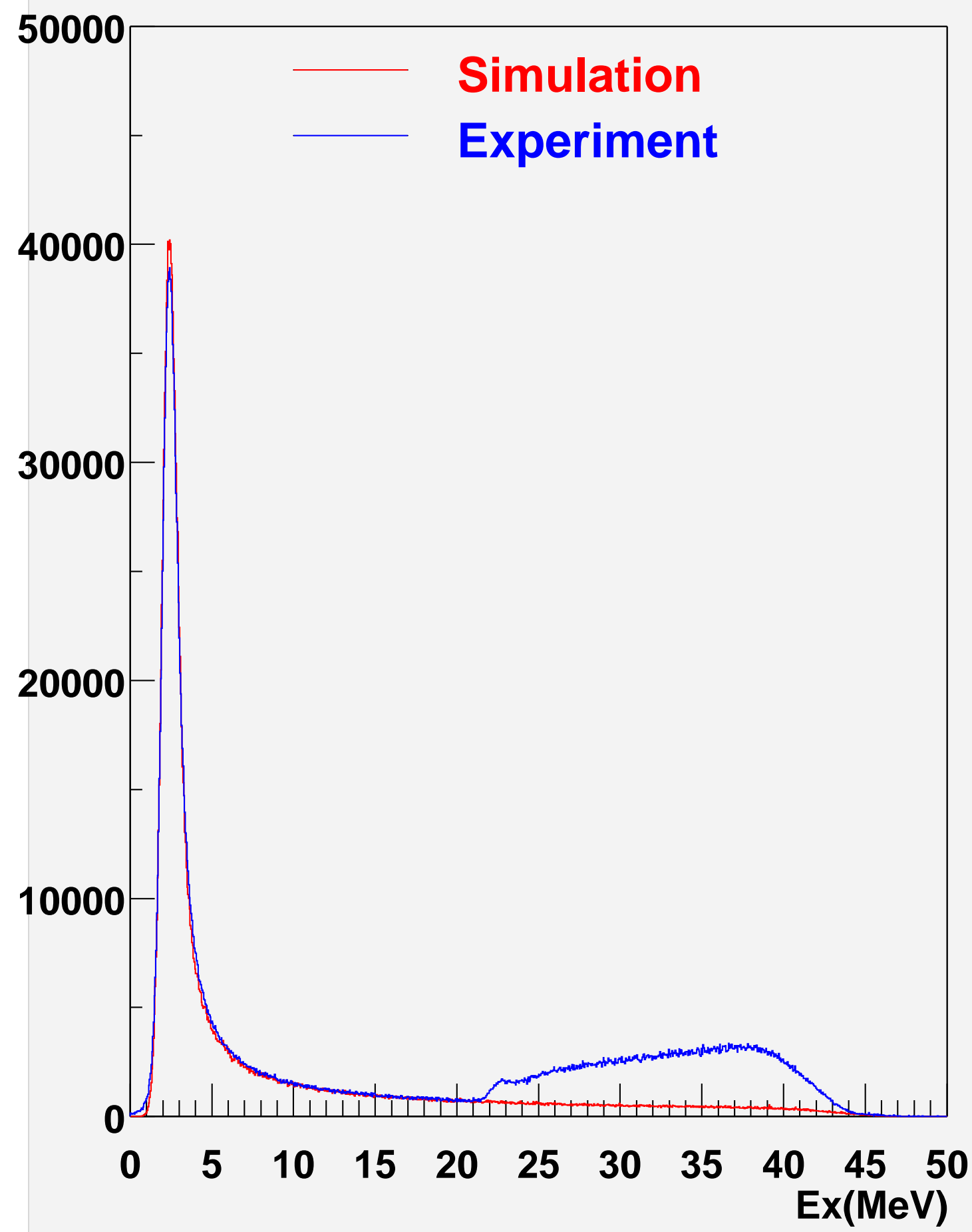

Figure 3-12: ${ }^{4} \mathrm{He}$ elastic radiative tail at $\mathrm{Q}^{2}=0.1(\mathrm{GeV} / \mathrm{c})^{2}$. 
the elastic and quasielastic spectrum in ${ }^{3} \mathrm{He}\left(\vec{e}, e^{\prime}\right)$ is shown in Figure 3-13. The slight discrepancy between data and simulation in the high excitation energy region at $Q^{2}$ $=0.1(\mathrm{GeV} / \mathrm{c})^{2}$ might be due to the fact that the spectrometer transfer functions were not optimized for low spectrometer momentum settings [78].
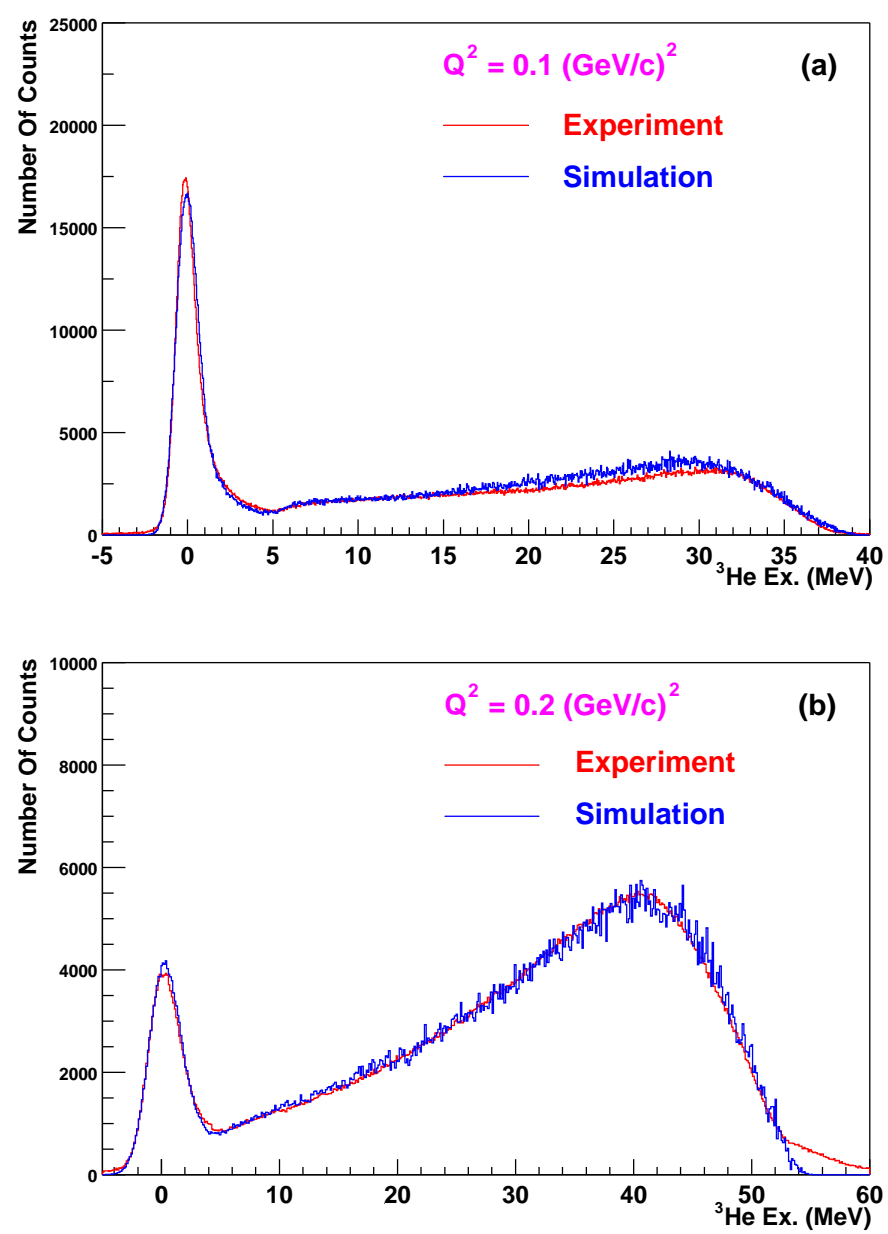

Figure 3-13: Comparison between data and simulation for both the elastic and quasielastic spectrum at $\mathrm{Q}^{2}=0.1$ and $0.2(\mathrm{GeV} / \mathrm{c})^{2}$.

A 2-MeV bin from $-1 \mathrm{MeV}$ to $1 \mathrm{MeV}$ in the excitation spectrum is used for the purpose of extracting $P_{b} P_{t}$. In reality, the value of $P_{b} P_{t}$ for each run is not explicitly extracted because the average $P_{b} P_{t}$ is the same for elastic scattering and quasielastic scattering, thus one can use the raw elastic asymmetry, raw quasielastic asymmetry and the simulated elastic asymmetry to determine the quasielastic asymmetry 
corrected for the beam and target polarizations directly as:

$$
A_{c o r}^{q e}=A_{e x p}^{q e} \times \frac{A_{s i m}^{e l}}{A_{e x p}^{e l}}
$$

However, it might still be of interest to obtain the average value of $P_{b} P_{t}$ to compare with the Møller measurement and NMR measurement. It was found to be $0.208 \pm 0.005$ [79], which is in good agreement with the value obtained from Møller measurement and NMR measurement, $0.215 \pm 0.013$.

\subsection{Elastic Radiative Tail Dilution Factor and Asym- metry}

With the precise knowledge of ${ }^{3} \mathrm{He}$ elastic form factors and the confidence from ${ }^{4} \mathrm{He}$ elastic radiative tail study, we can calculate the elastic radiative tail dilution factor and asymmetry to a high precision, which is very important in obtaining the physics quasielastic asymmetry from the raw asymmetry, especially near the twobody breakup threshold, where the spectrum is dominated by the elastic radiative tail.

The dilution factors and asymmetry for each bin is shown in Table 3.7 for $\mathrm{Q}^{2}=$ $0.1(\mathrm{GeV} / \mathrm{c})^{2}$, and in Table 3.8 for $\mathrm{Q}^{2}=0.2(\mathrm{GeV} / \mathrm{c})^{2}$.

\begin{tabular}{|c|c|c|}
\hline \hline Ex. $(\mathrm{MeV})$ & $\mathrm{R}^{\text {ert }}$ & $\mathrm{A}^{\text {ert }}(\%)$ \\
\hline 8.00 & $0.830 \pm 0.045$ & $5.934 \pm 0.130$ \\
13.00 & $0.326 \pm 0.017$ & $5.905 \pm 0.132$ \\
18.00 & $0.176 \pm 0.020$ & $5.875 \pm 0.131$ \\
23.00 & $0.109 \pm 0.017$ & $5.849 \pm 0.131$ \\
28.00 & $0.074 \pm 0.011$ & $5.816 \pm 0.130$ \\
33.00 & $0.058 \pm 0.004$ & $5.642 \pm 0.133$ \\
\hline \hline
\end{tabular}

Table 3.7: Elastic radiative tail dilution factors and asymmetry at $\mathrm{Q}^{2}=0.1(\mathrm{GeV} / \mathrm{c})^{2}$ 


\begin{tabular}{|c|c|c|}
\hline \hline Ex. $(\mathrm{MeV})$ & $\mathrm{R}^{\text {ert }}$ & $\mathrm{A}^{\text {ert }}(\%)$ \\
\hline 8.00 & $0.749 \pm 0.037$ & $5.426 \pm 0.179$ \\
13.00 & $0.268 \pm 0.015$ & $5.409 \pm 0.174$ \\
18.00 & $0.144 \pm 0.007$ & $5.384 \pm 0.179$ \\
23.00 & $0.086 \pm 0.004$ & $5.362 \pm 0.179$ \\
28.00 & $0.057 \pm 0.002$ & $5.332 \pm 0.175$ \\
33.00 & $0.040 \pm 0.001$ & $5.296 \pm 0.173$ \\
38.00 & $0.029 \pm 0.001$ & $5.241 \pm 0.169$ \\
43.00 & $0.023 \pm 0.001$ & $5.045 \pm 0.185$ \\
48.00 & $0.018 \pm 0.001$ & $4.817 \pm 0.230$ \\
\hline \hline
\end{tabular}

Table 3.8: Elastic radiative tail dilution factors and asymmetry at $\mathrm{Q}^{2}=0.2(\mathrm{GeV} / \mathrm{c})^{2}$

\subsection{Quasielastic Asymmetry Radiative Correction}

The radiative correction to quasielastic asymmetry is calculated as

$$
\Delta A^{q e}=A_{\text {born }}^{q e}-A_{\text {rad }}^{q e}
$$

where $A_{b o r n}^{q e}$ is the simulated asymmetry without radiative correction (thus using only Born cross section) and $A_{\text {rad }}^{q e}$ is the simulated asymmetry with both internal and external radiative corrections. The results are shown in Table 3.9 for $\mathrm{Q}^{2}=$ $0.1(\mathrm{GeV} / \mathrm{c})^{2}$, and in Table 3.10 for $\mathrm{Q}^{2}=0.2(\mathrm{GeV} / \mathrm{c})^{2}$.

\begin{tabular}{|c|c|}
\hline \hline Ex. $(\mathrm{MeV})$ & $\Delta A^{q e}(\%)$ \\
\hline 8.00 & $-0.092 \pm 0.020$ \\
13.00 & $-0.239 \pm 0.014$ \\
18.00 & $-0.129 \pm 0.010$ \\
23.00 & $-0.037 \pm 0.008$ \\
28.00 & $0.011 \pm 0.008$ \\
33.00 & $0.018 \pm 0.010$ \\
\hline \hline
\end{tabular}

Table 3.9: Radiative correction to quasielastic asymmetry at $\mathrm{Q}^{2}=0.1(\mathrm{GeV} / \mathrm{c})^{2}$ 


\begin{tabular}{|c|r|}
\hline \hline Ex. $(\mathrm{MeV})$ & \multicolumn{1}{|c|}{$\Delta A^{q e}(\%)$} \\
\hline 8.00 & $0.040 \pm 0.018$ \\
13.00 & $-0.213 \pm 0.034$ \\
18.00 & $-0.131 \pm 0.075$ \\
23.00 & $-0.064 \pm 0.012$ \\
28.00 & $0.023 \pm 0.006$ \\
33.00 & $0.029 \pm 0.012$ \\
38.00 & $0.041 \pm 0.015$ \\
43.00 & $0.034 \pm 0.018$ \\
48.00 & $0.048 \pm 0.020$ \\
\hline \hline
\end{tabular}

Table 3.10: Radiative correction to quasielastic asymmetry at $\mathrm{Q}^{2}=0.2(\mathrm{GeV} / \mathrm{c})^{2}$

\subsection{Bin-Averaging and Acceptance Effects}

The correction due to the bin-averaging and acceptance effects is calculated as

$$
\Delta A^{b i n}=A_{t h}-A_{s i m}
$$

where $A_{t h}$ is the asymmetry from the theoretical calculation, and $A_{s i m}$ is the simulated asymmetry using the same theoretical calculation. The simulation is performed under the experimental conditions, and $A_{\text {sim }}$ is calculated over the same energy bin as used for raw experimental asymmetry. Thus $A_{\text {sim }}$ includes both the spectrometer acceptance and bin-averaging effects.

The results are shown Table 3.11 for $\mathrm{Q}^{2}=0.1(\mathrm{GeV} / \mathrm{c})^{2}$, and in Table 3.12 for $\mathrm{Q}^{2}$ $=0.2(\mathrm{GeV} / \mathrm{c})^{2}$.

\subsection{Experimental Cross Section}

Although the major purpose of this experiment was to obtain the spin-dependent asymmetry, and thus no specific attention was paid to some factors crucial to a cross section measurement, such as target density and spectrometer acceptance, during the 


\begin{tabular}{|c|r|}
\hline \hline Ex. $(\mathrm{MeV})$ & \multicolumn{1}{|c|}{$\Delta A^{\text {bin }}(\%)$} \\
\hline 8.00 & $0.195 \pm 0.032$ \\
13.00 & $-0.030 \pm 0.015$ \\
18.00 & $0.000 \pm 0.009$ \\
23.00 & $0.029 \pm 0.023$ \\
28.00 & $0.043 \pm 0.009$ \\
33.00 & $0.082 \pm 0.009$ \\
\hline \hline
\end{tabular}

Table 3.11: Correction due to bin-averaging and acceptance effects at $\mathrm{Q}^{2}=$ $0.1(\mathrm{GeV} / \mathrm{c})^{2}$

\begin{tabular}{|c|r|}
\hline \hline Ex. $(\mathrm{MeV})$ & \multicolumn{1}{|c|}{$\Delta A^{\text {bin }}(\%)$} \\
\hline 8.00 & $0.522 \pm 0.014$ \\
13.00 & $0.017 \pm 0.011$ \\
18.00 & $-0.060 \pm 0.035$ \\
23.00 & $-0.063 \pm 0.021$ \\
28.00 & $-0.033 \pm 0.021$ \\
33.00 & $0.009 \pm 0.017$ \\
38.00 & $0.049 \pm 0.013$ \\
43.00 & $0.095 \pm 0.011$ \\
48.00 & $0.149 \pm 0.022$ \\
\hline \hline
\end{tabular}

Table 3.12: Correction due to bin-averaging and acceptance effects at $\mathrm{Q}^{2}=$ $0.2(\mathrm{GeV} / \mathrm{c})^{2}$

experiment, it is still of certain interest to extract the experimental cross section and compare it with theoretical calculations. However, it should be noted that this study is independent of the rest of the analysis.

We extract the experimental cross section based on the comparison between the experimental spectrum and the simulated spectrum with full Faddeev calculations for ${ }^{3} \mathrm{He}$ response functions. After the subtraction of elastic radiative tail, the ratio between the experimental spectrum and the simulated spectrum is proportional to the ratio between the experimental cross section and the theoretical calculation at 
the central kinematics; thus the experimental cross section can be determined as

$$
\sigma_{e x p}=\sigma_{\text {theory }} \times \frac{N_{e x p}-N_{e r t}}{N_{\text {sim }}-N_{e r t}}
$$

where $\sigma_{\text {exp }}$ is the experimental cross section, $\sigma_{\text {theory }}$ is the theoretical cross section, while $N_{e x p}, N_{\text {sim }}$ and $N_{\text {ert }}$ are the yields from the experimental spectrum, the simulated spectrum and the simulated elastic radiative tail, respectively. The experimental spectrum is corrected by deadtime and detector inefficiencies, and the correct target density and charge must be used in the simulation. In addition, as we previously noted, a major factor affecting the simulation is the spectrometer acceptance. We will discuss these important issues before presenting the results for the experimental cross section.

\subsection{1 ${ }^{3}$ He Density Determination}

${ }^{3} \mathrm{He}$ density inside the target chamber can be determined in the same way as the $\mathrm{N}_{2}$ pressure study discussed in Section 3.3 of this chapter. A few reference ${ }^{3} \mathrm{He}$ runs were taken at each kinematics during the experiment. The density of unpolarized ${ }^{3} \mathrm{He}$ gas in the reference cell was known precisely with the knowledge of its pressure and the reference cell temperature during the run, thus by comparing the ${ }^{3} \mathrm{He}$ elastic peak between a target ${ }^{3} \mathrm{He}$ run and a reference ${ }^{3} \mathrm{He}$ run, the target ${ }^{3} \mathrm{He}$ gas density can be

determined. The result is $(3.09 \pm 0.15) \times 10^{20} \mathrm{~cm}^{-3}$, which agrees with the result from a second technique, called the pressure broadening measurement [59], to within $5 \%$.

\subsubsection{Deadtime and Detector Inefficiencies}

The deadtime for each run can be easily obtained from the number of events written to a scaler counter and the number of events actually recorded by ESPACE

$$
\text { Deadtime }=1-\frac{\text { Scaler Counts }}{\text { ESPACE Counts }}
$$


and thus it is corrected run-by-run.

The trigger inefficiency refers to the percentage of good events that do not fire both scintillator planes and thus trigger a signal. The trigger inefficiency can be approximated by one minus the ratio of the T1 events to the sum of $\mathrm{T} 1$ events and T2 events times the prescaler factor, which was 25 during the entire experiment

$$
\text { Trigger Inefficency } \approx 1-\frac{T 1}{T 1+25 \times T 2}
$$

The trigger inefficiency for this experiment was found to be around $3-5 \%$ and is also correctable by adding the T1 spectrum and twenty five times T2 spectrum together to form the experimental spectrum.

The VDC inefficiency is the percentage of good events that do not form a track because of the inefficiencies of the VDC wires. It was determined to be less than $1 \%$ for the entire experiment, which shows that the VDC performed very well during our experiment.

\subsubsection{Spectrometer Acceptance}

The spectrometer acceptance is defined through spectrometer transfer functions, which are used to transfer an event from the target region to the entrance and exit planes of each magnet, and the magnet apertures. It is very difficult to study the uncertainty in the transfer functions, but we can study the effects of magnet apertures by placing the same magnet aperture cuts on both data and simulation and then varying the aperture cuts to see the agreement between data and simulation. A norminal 10\% uncertainty in spectrometer acceptance was assigned from this study.

\subsubsection{Results for Experimental Cross Section}

The experimental cross sections for both $\mathrm{Q}^{2}$-values are shown in Figure 3-14 together with the Faddeev calculations which include both FSI and MEC effects. The sta- 
tistical error is negligible and the systematic error is mainly due to the uncertainty in ${ }^{3} \mathrm{He}$ density determination, which is $5 \%$, and the uncertainty in the spectrometer acceptance, which we assume to be $10 \%$.

The unsatisfactory agreement in the high excitation energy region at $Q^{2}=0.1$ $(\mathrm{GeV} / \mathrm{c})^{2}$ might be due to the fact that the spectrometer transfer functions were not optimized for low spectrometer momentum settings [78]. To get a really good understanding of the spectrometer acceptance, more sophisticated analysis using Rfunctions might be necessary. 

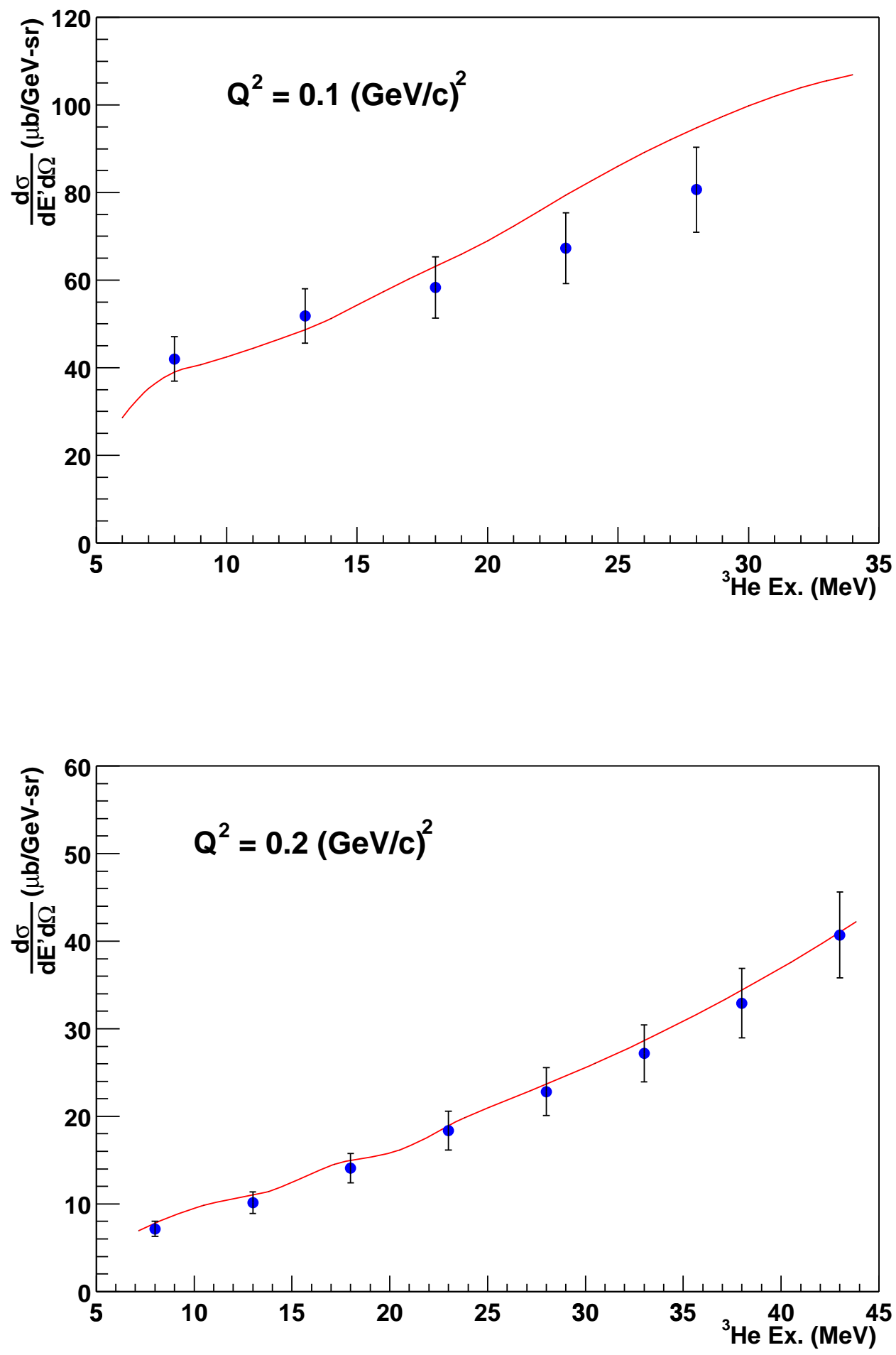

Figure 3-14: The comparison between experimental cross section and theoretical cross section with full Faddeev calculations at $Q^{2}=0.1$ (above) and $0.2(\mathrm{GeV} / \mathrm{c})^{2}$ (below). The statistical error of the data is negligible while the systematic error is due to the uncertainty in the determination of ${ }^{3} \mathrm{He}$ target density and spectrometer acceptance. 


\section{Chapter 4}

\section{Systematic Uncertainties}

For a typical asymmetry measurement, some major systematic uncertainties come from various false asymmetries, the sources of which include the helicity-correlated beam current shift, the helicity-correlated beam position shift, helicity-correlated detector inefficiencies, and empty target and $\mathrm{N}_{2}$ false asymmetries. Empty target and $\mathrm{N}_{2}$ false asymmetries were shown to be consistent with zero in Chapter 3, so we will not discuss them here.

Other systematic uncertainties come from the extraction of physics asymmetry from raw experimental asymmetry, which includes empty target and $\mathrm{N}_{2}$ background subtraction, elastic radiative tail subtraction, radiative correction on quasielastic asymmetry and correction for bin-averaging and acceptance effects. Systematic uncertainties due to empty target and $\mathrm{N}_{2}$ dilution factors can be obtained by studying the variation of the dilution factors from run to run. Systematic uncertainties due to other factors must be understood through the simulation program by systematically varying different inputs to the simulation, which include elastic form factors, quasielastic response functions, the thickness of all materials that incoming and scattered electrons pass through, and the spectrometer acceptance. Finally, a potential contribution to systematic uncertainties is the pion contamination which, as we will show, is completely negligible in our analysis because of the kinematics and good 
particle identification (PID).

\subsection{Systematic Uncertainties from False Asymme- tries}

\subsubsection{Helicity-Correlated Beam Current Shift}

There are two practical reasons to study the helicity-correlated beam current shift. First, it has been known that the beam energy varies slightly with the beam current, which is called the beam loading effect. Thus if there is a helicity-correlated beam current difference, there will be a false asymmetry contribution to the experimental asymmetry due to the beam energy shift, since the physics cross section is energy dependent, especially for the elastic scattering process. Secondly, as we discussed in Chapter 3, the experimental data have to be corrected for the charge before the raw asymmetry is formed. The charge is determined from the scaler readout of BCMs and $\mathrm{BCM}$ calibration constants, and it is a well-known fact that BCM current calibration constants are dependent on the beam current. Thus if there is a helicity-correlated beam current and we use the same BCM calibration constants for both helicities, we will introduce a current-related false asymmetry to the experimental asymmetry.

During the experiment two independent methods were used to check the helicitycorrelated beam current shift. One method calculated the beam current asymmetry directly from the readouts of all eight BCMs, and the results are shown in Figure 4-1

for a typical run. The second method used an independent DAQ system from the Happex experiment to perform a direct on-line measurement of the current asymmetry, and the result is shown in Figure 4-2 for the same run. Both methods showed that the beam current asymmetry was on the order of $2 \mathrm{ppm}$ during the experiment, thus the induced false asymmetry is completely negligible. 


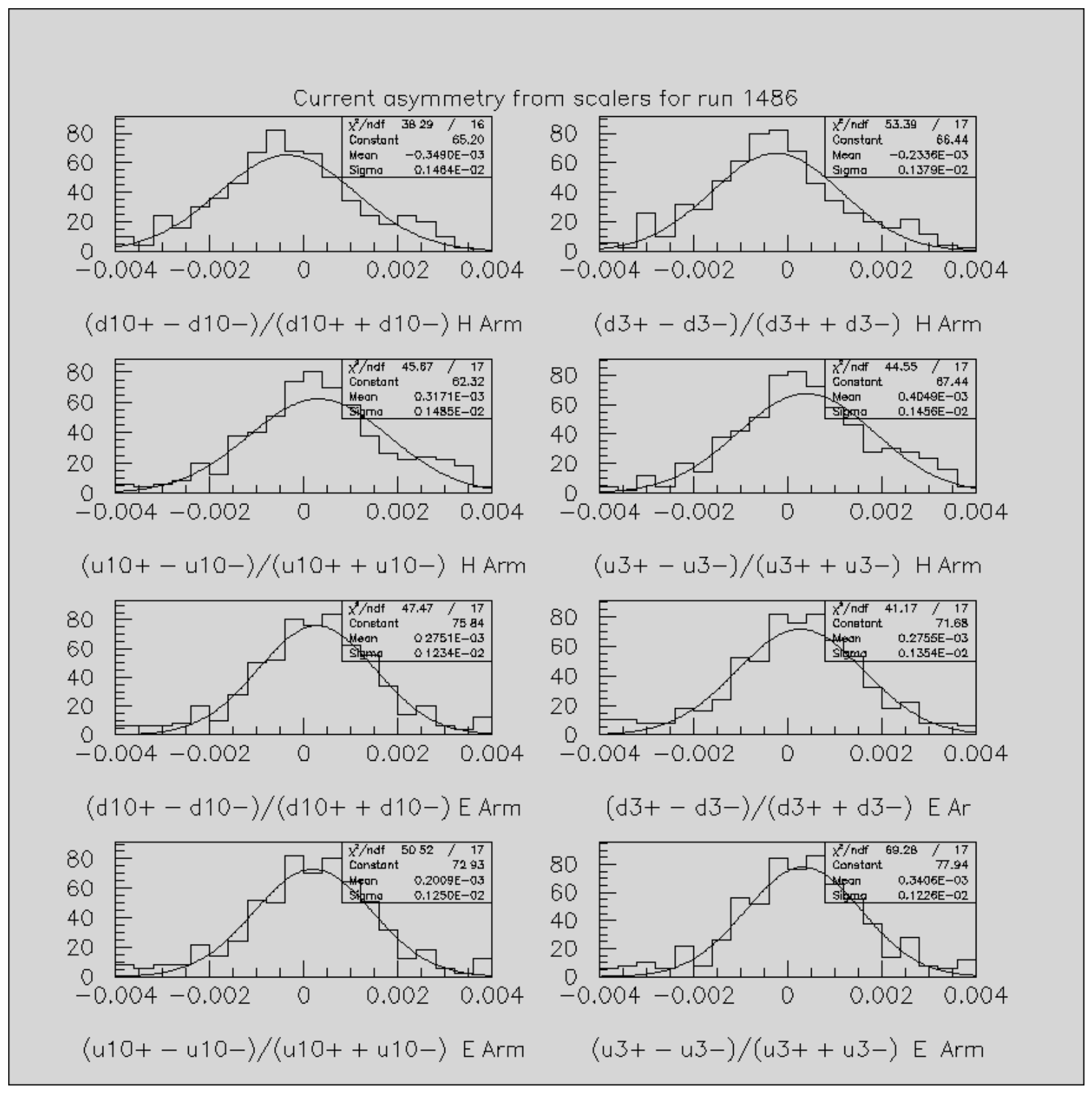

Figure 4-1: Beam current asymmetry distribution for a typical run from the scaler readouts of all right BCMs. As can be seen, the beam current asymmetry is consistent with zero. 


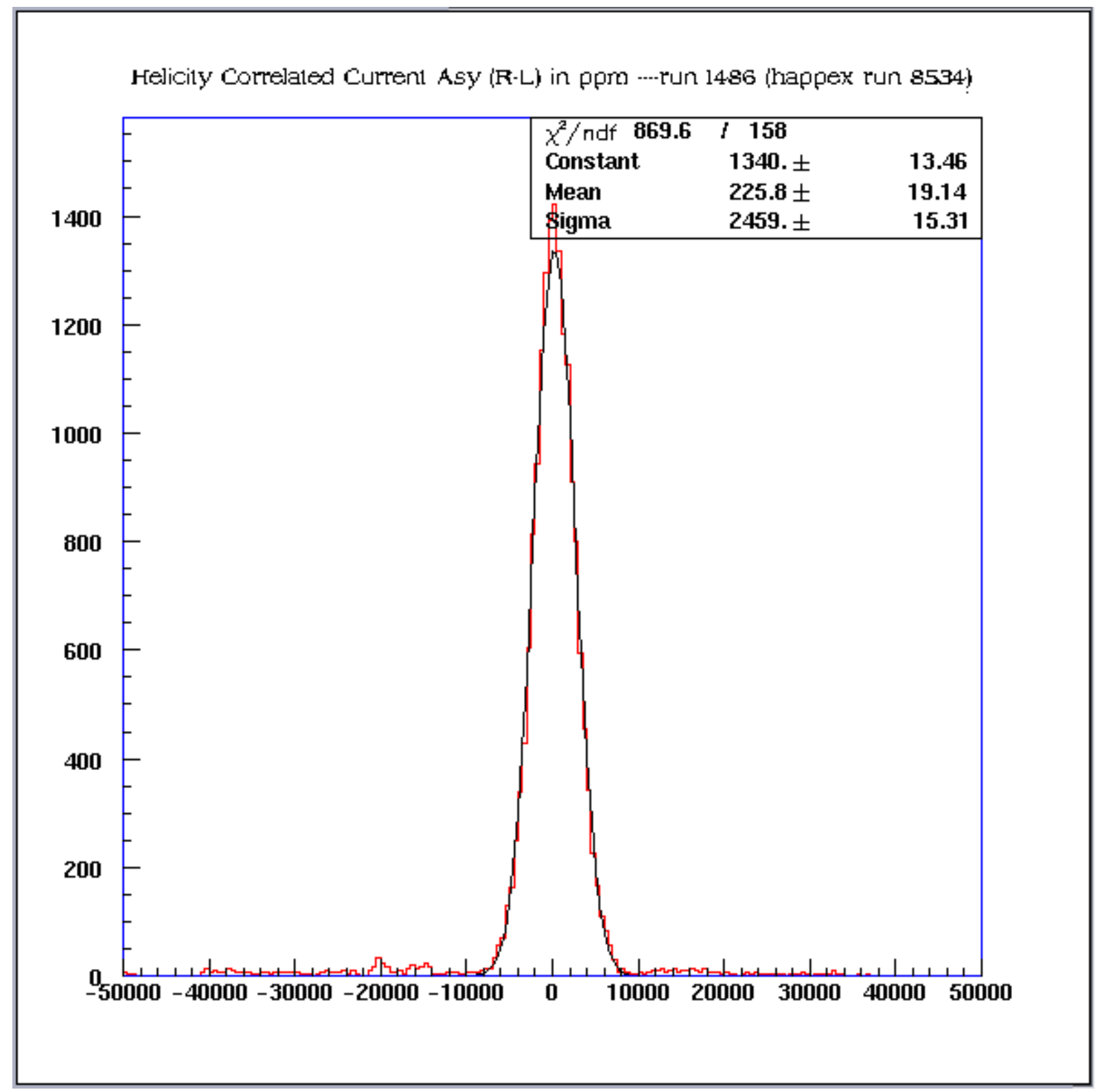

Figure 4-2: Beam current asymmetry distribution for the same run from an independent DAQ for the Happex experiment. As can be seen, the beam current asymmetry is consistent with zero. 


\subsubsection{Helicity-Correlated Beam Position Shift}

As we mentioned earlier, the empty target yield was dependent on the central beam position on the target, and a nominal beam position was chosen to minimize the empty target yield before the experiment was conducted. If there was a helicitycorrelated beam position shift, this would introduce a false asymmetry to the experimental asymmetry because the empty target yields would then be different for the two helicities.

We plot the central beam positions for different helicities and their difference in Figure 4-3 for some runs at electron arm $Q^{2}=0.3(\mathrm{GeV} / \mathrm{c})^{2}$, which corresponds to hadron arm $Q^{2}=0.2(\mathrm{GeV} / \mathrm{c})^{2}$. As can be seen, the helicity-correlated beam position shift was consistent with zero. The resulting false asymmetry was estimated to be less than $5 \mathrm{ppm}$, which is negligible compared to the statistical accuracy of the experimental asymmetry.

\subsubsection{Data Acquisition Deadtime}

There are two kinds of data acquisition (DAQ) deadtime: the electronics deadtime and the computer deadtime. The electronics deadtime is due to the time duration, $\tau$, of the logic pulse passed to the scaler. If two independent pulses arrive at the scaler within a time interval shorter than $\tau$, then only one pulse will be recorded. Since $\tau$ was about $100 \mathrm{~ns}$ and the maximum rates for this experiment was a few $\mathrm{kHz}$, the electronics deadtime is less than $1 \%$ and thus negligible. The computer deadtime is due to the fact that the data acquisition system can only process up to about 1250 events per second, thus a lot of events will not be recorded if the event rates are too high. The deadtime in this experiment was typically $20-30 \%$.

From the above discussion it should be clear that the DAQ deadtime is strongly correlated with event rate, and for an asymmetry measurement, the event rate is obviously helicity-dependent (otherwise there would not be any asymmetry), thus 

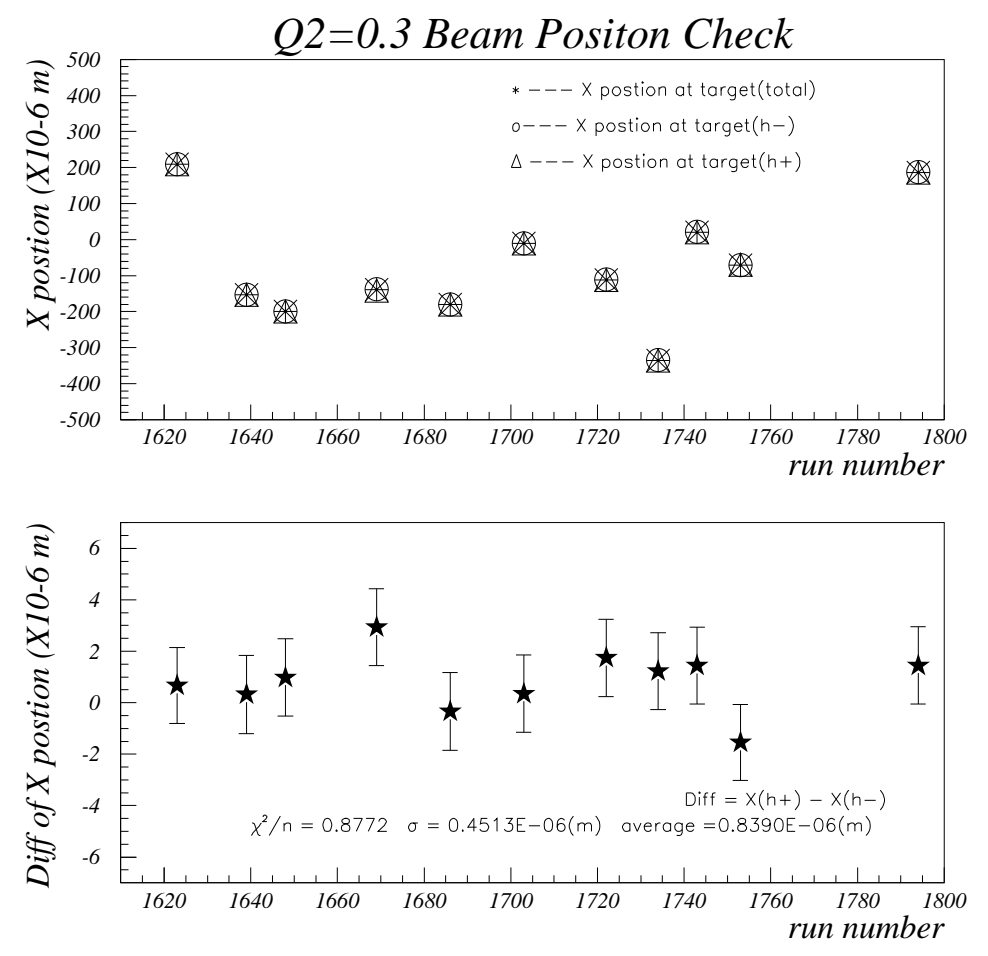

Figure 4-3: The central beam positions (upper panel) for helicity plus and minus and their difference (lower panel) for some runs at electron $\operatorname{arm} Q^{2}=0.3(\mathrm{GeV} / \mathrm{c})^{2}$, which corresponds to hadron arm $Q^{2}=0.2(\mathrm{GeV} / \mathrm{c})^{2}$. 


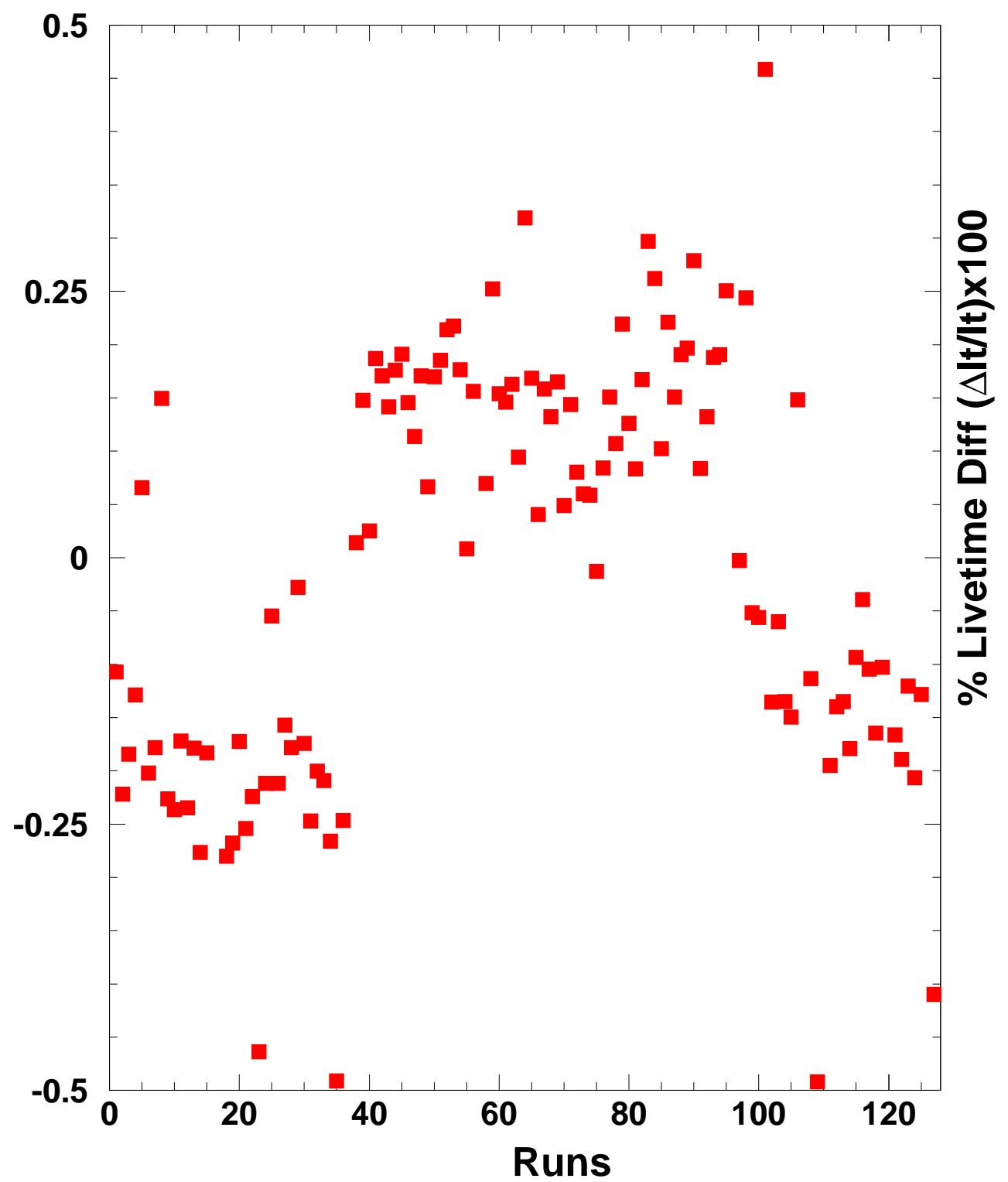

Figure 4-4: Livetime difference between the two beam helicities. The change of sign on the two edges is due to the change of the beam half-wave plate status. 
there will necessarily be a non-negligible false asymmetry due to DAQ deadtime. The livetime (which is simply one minus deadtime) difference between the two beam helicities is plotted in Figure 4-4 for some runs and on average it is about $0.25 \%$ (note that the change of sign is due to the change of the beam half-wave plate status).

Thus there will be a false asymmetry due to the helicity-correlated deadtime which, fortunately, is easily correctable since the deadtime can be obtained for each helicity. This is the reason why we always correct for both charge and deadtime in forming the experimental raw asymmetry.

\subsubsection{Trigger Efficiency}

As discussed in Chapter 2, a good event is defined as one that passes both scintillator planes, S1 and S2. However, not all good events will fire the phototubes on the two edges of each scintillator paddle and generate a trigger signal, because of the inefficiencies of the phototubes. For a cross section measurement, the trigger inefficiency must be studied carefully and the experimental yield must be corrected for this inefficiency. For an asymmetry measurement, it is not necessary to correct the yield in this way, but if the trigger efficiency depends on the electron helicity, it would introduce false asymmetry into the physics asymmetry.

The trigger efficiency depends on the scintillator efficiency of each paddle in S1 and S2 planes in a rather complicated way [79]. However, for the purpose of false asymmetry study, it is sufficient to check the helicity dependence of the scintillator efficiency paddle-by-paddle. In Figure 4-5 the difference in scintillator efficiency between the two beam helicities for each paddle is shown for all runs at $\mathrm{Q}^{2}=0.2$ $(\mathrm{GeV} / \mathrm{c})^{2}$. It is clear that the scintillator efficiency is helicity-independent and the introduced false asymmetry is consistent with zero. 

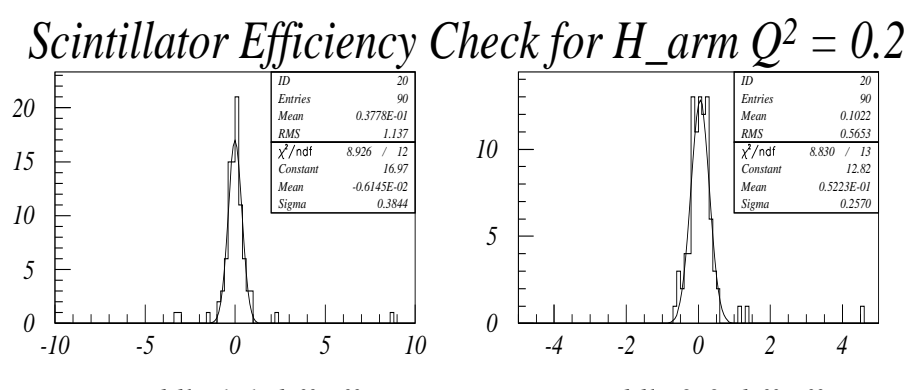

paddle 1-1 diff-eff

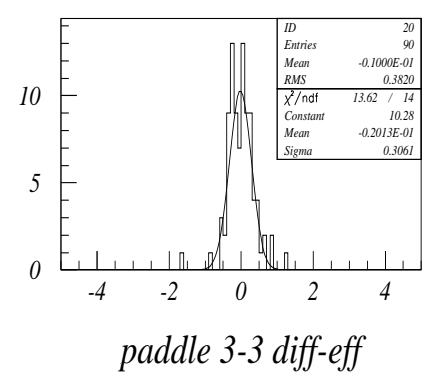

paddle 2-2 diff-eff
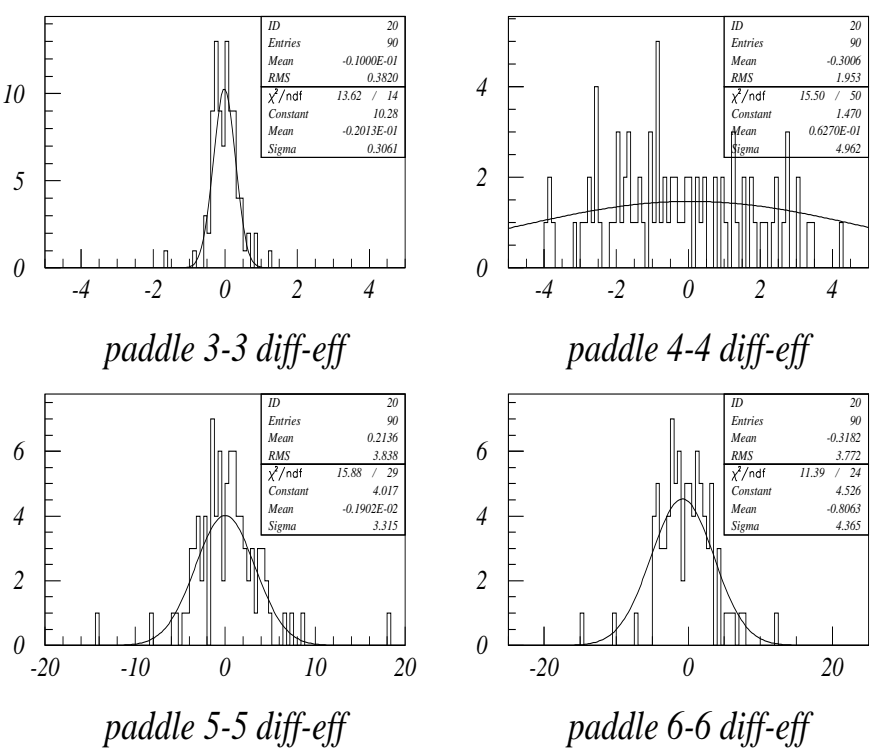

Figure 4-5: Difference in scintillator efficiency between the two beam helicities for each paddle and for all runs at $\mathrm{Q}^{2}=0.2(\mathrm{GeV} / \mathrm{c})^{2}$. 


\subsubsection{VDC Efficiency}

When a charged particle passes through a wire chamber, typically four or five wires will fire instead of one wire. Thus, it is very complicated to quantify the overall VDC efficiency since it involves many different wires. In practice, another quantity, the wire efficiency, is commonly used. The wire efficiency is defined as the probability for a wire to fire when its neighboring wires fire and form a cluster. It is clear that the VDC performs well if each wire functions properly and thus the wire efficiency is an excellent substitute for the overall VDC efficiency.

The wire efficiency was determined to be above $99 \%$ for most wires, which shows that the VDC performed very well during our experiment. The false asymmetry of the wire efficiency with respect to the beam helicities was consistent with zero, as can be seen in Figure 4-6.

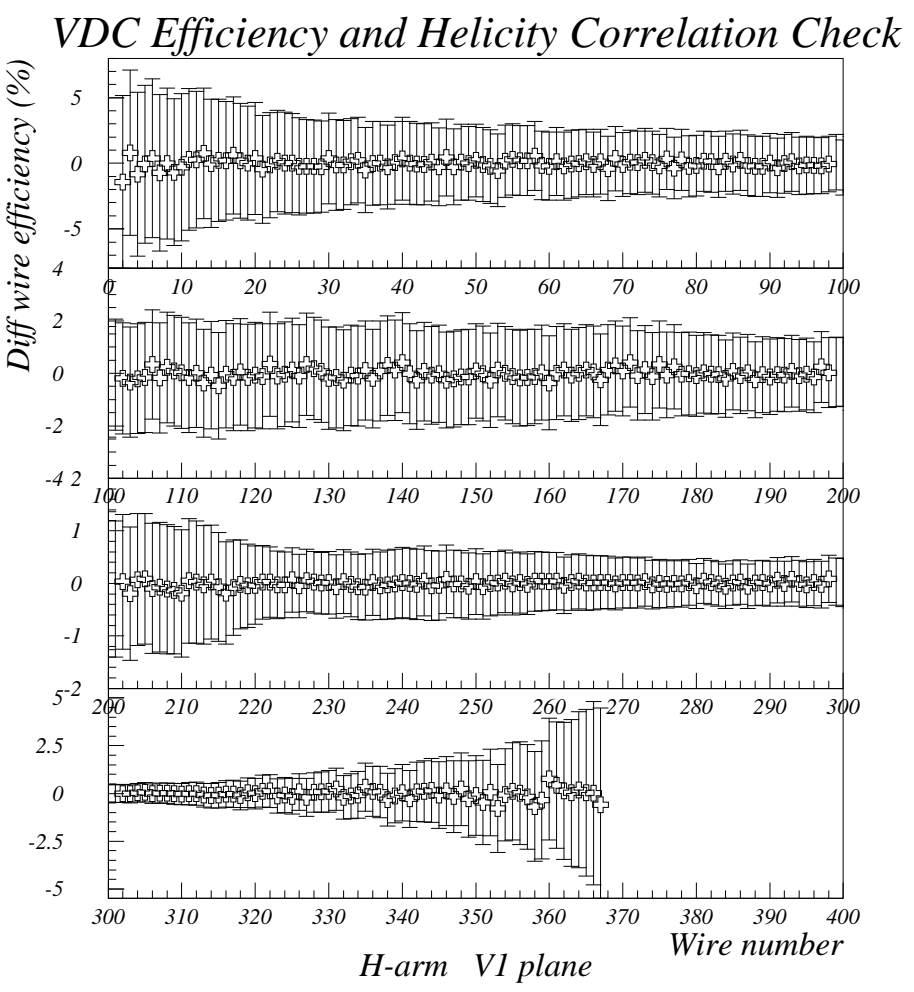

Figure 4-6: Helicity dependence of the wire efficiency for each wire of the four VDC chambers. 


\subsection{Systematic Uncertainties from Background Sub- traction}

\subsubsection{Empty Target and $\mathrm{N}_{2}$ Dilution Factors}

As was described in Chapter 3 , to obtain the empty target or $\mathrm{N}_{2}$ dilution factors, all empty or $\mathrm{N}_{2}$ runs in the same kinematics were added together to improve statistical accuracy. However, since different runs were taken at slightly different experimental conditions, for example, the beam position on the target might differ slightly from run to run, the dilution factors might be different for each run. Also there might be some error in measuring $\mathrm{N}_{2}$ pressure in the reference cell for different reference $\mathrm{N}_{2}$ runs. Thus we must assign a systematic uncertainty to the dilution factors. In Figure 4-7 the overall empty target dilution factors obtained using different empty target runs are plotted together. The variation from run to run is within $\pm 15 \%$. In Figure $4-8$ the overall $\mathrm{N}_{2}$ dilution factors obtained using different $\mathrm{N}_{2}$ runs are plotted together. The variation from run to run is typically within $\pm 3 \%$. We assign corresponding systematic uncertainties to empty target and $\mathrm{N}_{2}$ dilution factors.

\subsubsection{Pion Contamination}

Pion contamination can be a serious problem for electron scattering experiment, especially for high energy deep inelastic scattering. However its effect is expected to be very small for our kinematics.

A particle identification (PID) package consisting of a pair of preshower and shower counters and a Čerenkov detector was employed in this experiment to separate electrons from pions. The resulting PID cut is very effective and the pion contamination was found to be less than $0.1 \%$; thus its effect is completely negligible. 


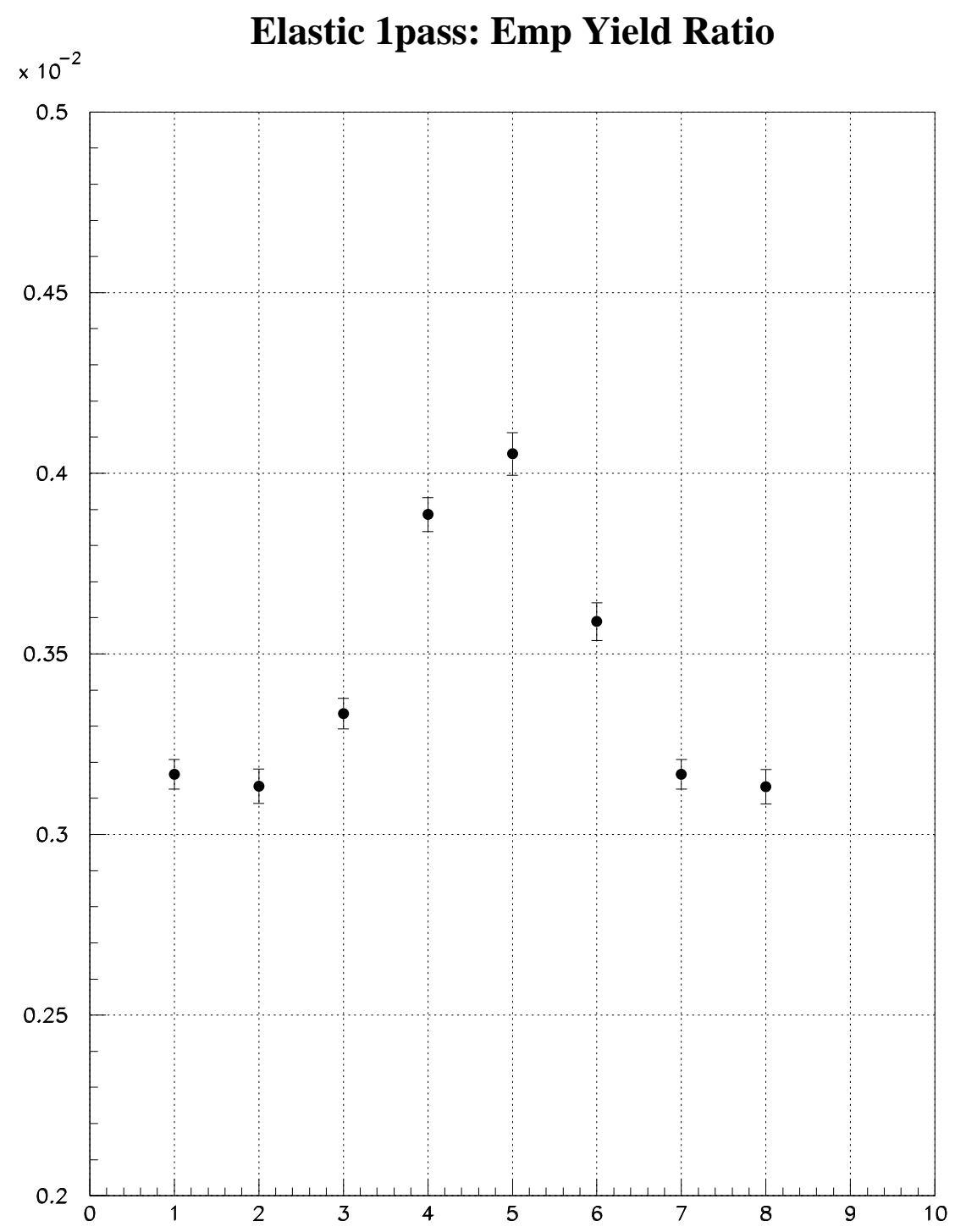

Figure 4-7: Variation of the overall empty target dilution factor obtained using different empty target runs. 


\section{Elastic 1pass: N2 Yield Ratio}

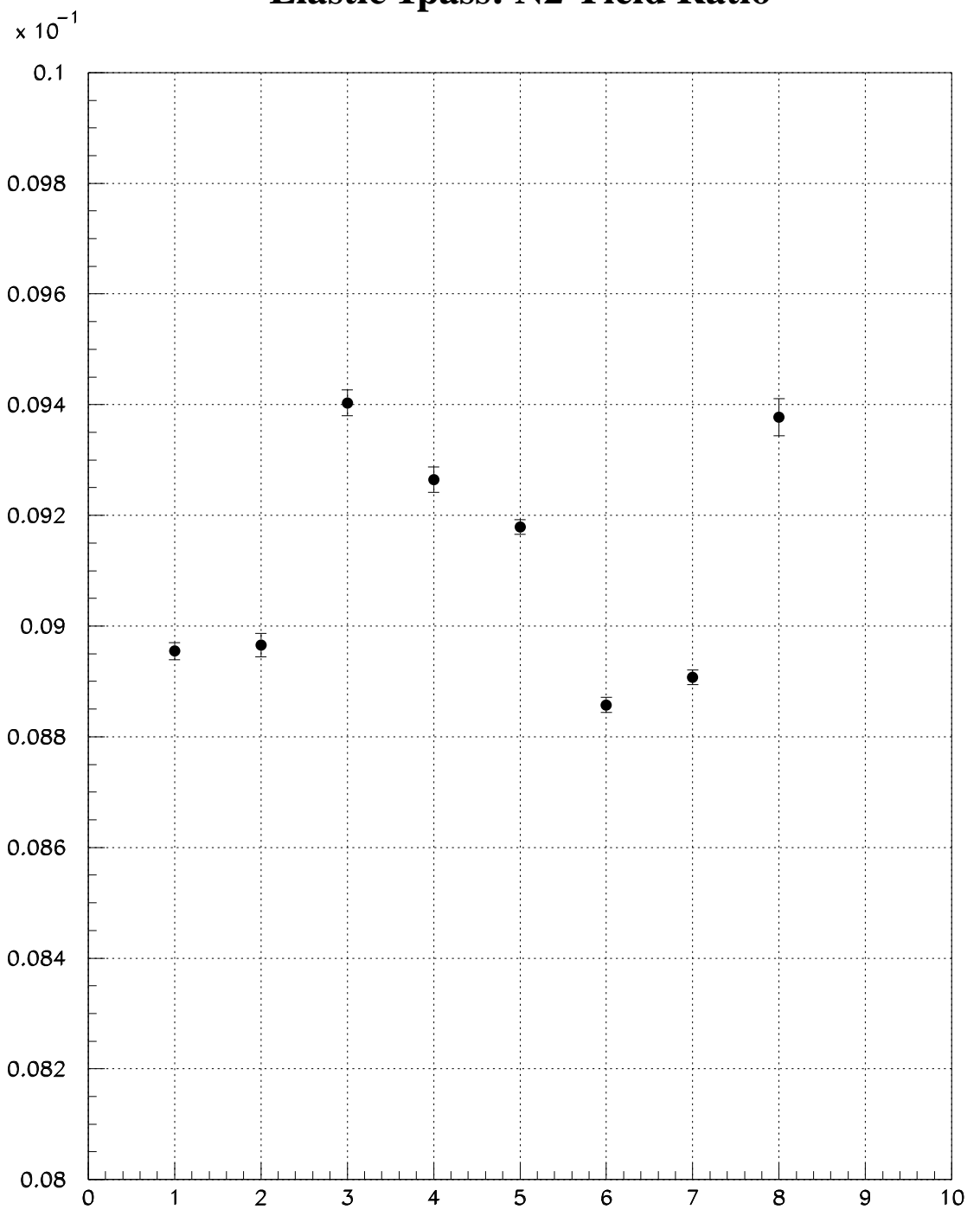

Figure 4-8: Variation of the overall $\mathrm{N}_{2}$ dilution factor obtained using different $\mathrm{N}_{2}$ runs. 


\subsection{Systematic Uncertainties from Simulation}

As discussed in Chapter 3, to extract the physics asymmetry from the experimental raw asymmetry, many factors must be obtained from simulation. These factors include the elastic radiative tail dilution factor and asymmetry, the radiative correction to the quasielastic asymmetry and the correction for bin-averaging and acceptance effects.

In this section we will discuss various inputs to the simulation and how uncertainties in each input affect one or more of the above factors. Systematic uncertainties of each factor due to all inputs will be added in quadrature and then propagated through Eq. (3.3) to obtain the systematic uncertainty of the physics asymmetry due to this factor, which will be shown in Chapter 5.

\subsubsection{Elastic Form Factors}

${ }^{3} \mathrm{He}$ elastic form factors were used as inputs to the simulation in two ways. First, they were used to simulate the elastic asymmetry and determine the product of beam and target polarizations. Secondly, a range of ${ }^{3} \mathrm{He}$ elastic form factors were used to simulate the elastic radiative tail under the quasielastic spectrum and calculate both the asymmetry and dilution factors of the elastic radiative tail.

In [77], ${ }^{3} \mathrm{He}$ elastic form factors were measured to an accuracy of $2 \%$, thus we vary them by $\pm 2 \%$ in the simulation to study their effects on various factors.

\subsubsection{Quasielastic Response Functions}

${ }^{3} \mathrm{He}$ quasielastic response functions obtained from full Faddeev calculations with AV18 as the Nucleon-Nucleon potential and including both FSI and MEC effects were used in the simulation with a three-fold purpose. First, they were used to get the elastic radiative tail dilution factors by comparing the elastic radiative tail and pure quasielastic spectrum with radiative correction. Secondly, they were used to get the radiative 
correction to the quasielastic asymmetry by subtracting the simulated quasielastic asymmetry with radiative correction from the simulated quasielastic asymmetry without radiative correction. Finally, they were also used to get the correction due to binaveraging and acceptance effects by subtracting the simulated quasielastic asymmetry without radiative correction from the theoretical quasielastic asymmetry.

It is hard to estimate the uncertainties of quasielastic response functions in the threshold region, especially the spin-dependent ones, since the currently available experimental data have too large error bars. Thus we simply vary the response functions by $\pm 10 \%$ and see the effects, which we believe is a very conservative estimate.

\subsubsection{Thickness of External Radiative Material}

The thickness of the materials that electrons pass through is a very important input to the simulation because they will directly affect the external radiative correction, which includes most probably energy loss, energy straggling and external bremsstrahlung. The dominant contribution to external radiative correction comes from the wall thickness of the target cell. The wall thickness was measured using laser interferometry [80] at different points along the beam direction. The results are shown in Table 4.1. The average wall thickness was determined to be $1.20 \pm 0.02 \mathrm{~mm}$. Thus in the simulation we vary the material thickness by $\pm 2 \%$ and study the effect on various factors.

\subsubsection{Spectrometer Acceptance}

For an asymmetry experiment, most of the systematic uncertainties due to spectrometer acceptance will cancel out when forming the ratio. However, it is still necessary to understand the residual systematic uncertainties in such a high precision measurement as our experiment. In addition, in our case, the elastic asymmetry, elastic radiative tail asymmetry, radiative correction and the correction due to bin-averaging and acceptance effects all have direct dependence on the spectrometer acceptance. As was mentioned, the spectrometer acceptance is not very well understood in Hall A 


\begin{tabular}{|c|c|}
\hline \hline $\begin{array}{c}\text { Relative Position } \\
\text { (inch) }\end{array}$ & $\begin{array}{c}\text { Thickness } \\
\mathrm{mm}\end{array}$ \\
\hline 5.0 & 1.237 \\
4.0 & 1.208 \\
2.2 & 1.198 \\
1.7 & 1.218 \\
-0.5 & 1.230 \\
-1.8 & 1.196 \\
-3.0 & 1.187 \\
-4.0 & 1.184 \\
\hline
\end{tabular}

Table 4.1: Target wall thickness at different positions along the beam line relative to the target center.

and thus we made a very conservative estimate of its effect by calculating each factor using two sets of simulations, one with the original geometric magnet apertures and the other with much improved physical magnet apertures, as described in Chapter 3, and taking the difference of them as the systematic uncertainty of this factor due to acceptance. This sounds not to be a good idea because the simulated spectrums are wildly different with these two sets of magnet apertures, but as it turns out, the effect on various factors that go into the physics asymmetry is very small. This will be clear when we look at the results in Chapter 5 . 


\section{Chapter 5}

\section{Results and Discussion}

In this chapter we will first present the results for the physics asymmetries at both $Q^{2}=0.1$ and $0.2(\mathrm{GeV} / \mathrm{c})^{2}$ and their systematic uncertainties. We then compare the results with different theoretical calculations, including plane wave impulse approximation (PWIA) calculations and state-of-the-art non-relativistic Faddeev calculations with final-state interactions (FSIs) and meson-exchange currents (MECs), and discuss various theoretical uncertainties. With the insights gained from this comparison, we will investigate two extensions to the current framework of non-relativistic Faddeev calculations, three-nucleon forces (3NFs) and relativistic corrections, and study their effects on our results. Finally we will summarize the results and draw the conclusion.

\subsection{Physics Asymmetry and Comparison with The- oretical Calculations}

The physics asymmetries are shown in Table 5.1 for $Q^{2}=0.1(\mathrm{GeV} / \mathrm{c})^{2}$ and Table 5.2 for $Q^{2}=0.2(\mathrm{GeV} / \mathrm{c})^{2}$, together with their statistical and systematic uncertainties. The systematic uncertainties include contributions from the determination of $P_{b} P_{t}$, empty target and $N_{2}$ background subtraction, elastic radiative tail subtraction, radiative correction and the correction of spectrometer acceptance and bin-averaging 
effects. A careful analysis of various systematic uncertainties was carried out and has been discussed in Chapter 4.

\begin{tabular}{|ccccccc|}
\hline \hline $\begin{array}{c}E_{x} \\
(\mathrm{MeV})\end{array}$ & $\begin{array}{c}A \pm \delta^{\text {stat }} \\
(\%)\end{array}$ & $\begin{array}{c}\delta^{\text {pol }} \\
(\%)\end{array}$ & $\begin{array}{c}\delta^{\text {dil }} \\
(\%)\end{array}$ & $\begin{array}{c}\delta^{\text {ert }} \\
(\%)\end{array}$ & $\begin{array}{c}\delta^{r c} \\
(\%)\end{array}$ & $\begin{array}{c}\delta^{a c c} \\
(\%)\end{array}$ \\
\hline 8.0 & $3.602 \pm 0.157$ & 0.153 & 0.048 & 0.105 & 0.020 & 0.032 \\
13.0 & $1.666 \pm 0.100$ & 0.073 & 0.021 & 0.061 & 0.014 & 0.015 \\
18.0 & $1.399 \pm 0.082$ & 0.050 & 0.012 & 0.076 & 0.010 & 0.009 \\
23.0 & $1.553 \pm 0.071$ & 0.043 & 0.009 & 0.066 & 0.008 & 0.023 \\
28.0 & $1.768 \pm 0.063$ & 0.043 & 0.008 & 0.042 & 0.008 & 0.009 \\
33.0 & $1.756 \pm 0.066$ & 0.039 & 0.007 & 0.016 & 0.010 & 0.009 \\
\hline
\end{tabular}

Table 5.1: The physics asymmetry as a function of excitation energy $\left(E_{x}\right)$ at $Q^{2}=0.1$ $(\mathrm{GeV} / \mathrm{c})^{2}$, together with its statistical and systematic uncertainties. The systematic uncertainties include contributions from the determination of $P_{b} P_{t}\left(\delta^{p o l}\right)$, target wall and $N_{2}$ dilution $\left(\delta^{d i l}\right)$, elastic radiative tail subtraction $\left(\delta^{\text {ert }}\right)$, radiative correction $\left(\delta^{r c}\right)$ and the correction of spectrometer acceptance and bin-averaging effects $\left(\delta^{a c c}\right)$. The physics asymmetry $(A)$ and statistical uncertainties $\left(\delta^{\text {stat }}\right)$ are also shown.

A comparison between the physics asymmetries and various theoretical calculations is shown in Fig. 5-1. The uncertainty in determining the excitation energy is about $0.4 \mathrm{MeV}$ at $Q^{2}=0.1(\mathrm{GeV} / \mathrm{c})^{2}$, and $1.0 \mathrm{MeV}$ at $Q^{2}=0.2(\mathrm{GeV} / \mathrm{c})^{2}$, dominated by the uncertainty in the beam energy. The vertical error bars on the data are the statistical and systematic errors added in quadrature. All theoretical calculations were performed using AV18 [26] as the $N N$ interaction potential and the Höhler nucleon form factor parameterization[81]. Plane wave impulse approximation (PWIA) calculations $[9,82]$ are shown as dot-dashed lines. Non-relativistic Faddeev calculations with FSI only [48] are shown as dashed lines. Non-relativistic Faddeev calculations which include both FSI and MEC [49] are shown as dotted lines without the inclusion of the $\Delta$ isobar current, and solid lines with the inclusion of the $\Delta$ isobar current.

As can be seen from Figure 5-1, the agreement between PWIA calculations and the data is very poor at both kinematics, which confirms the intuition that at low $\mathrm{Q}^{2}$ and 

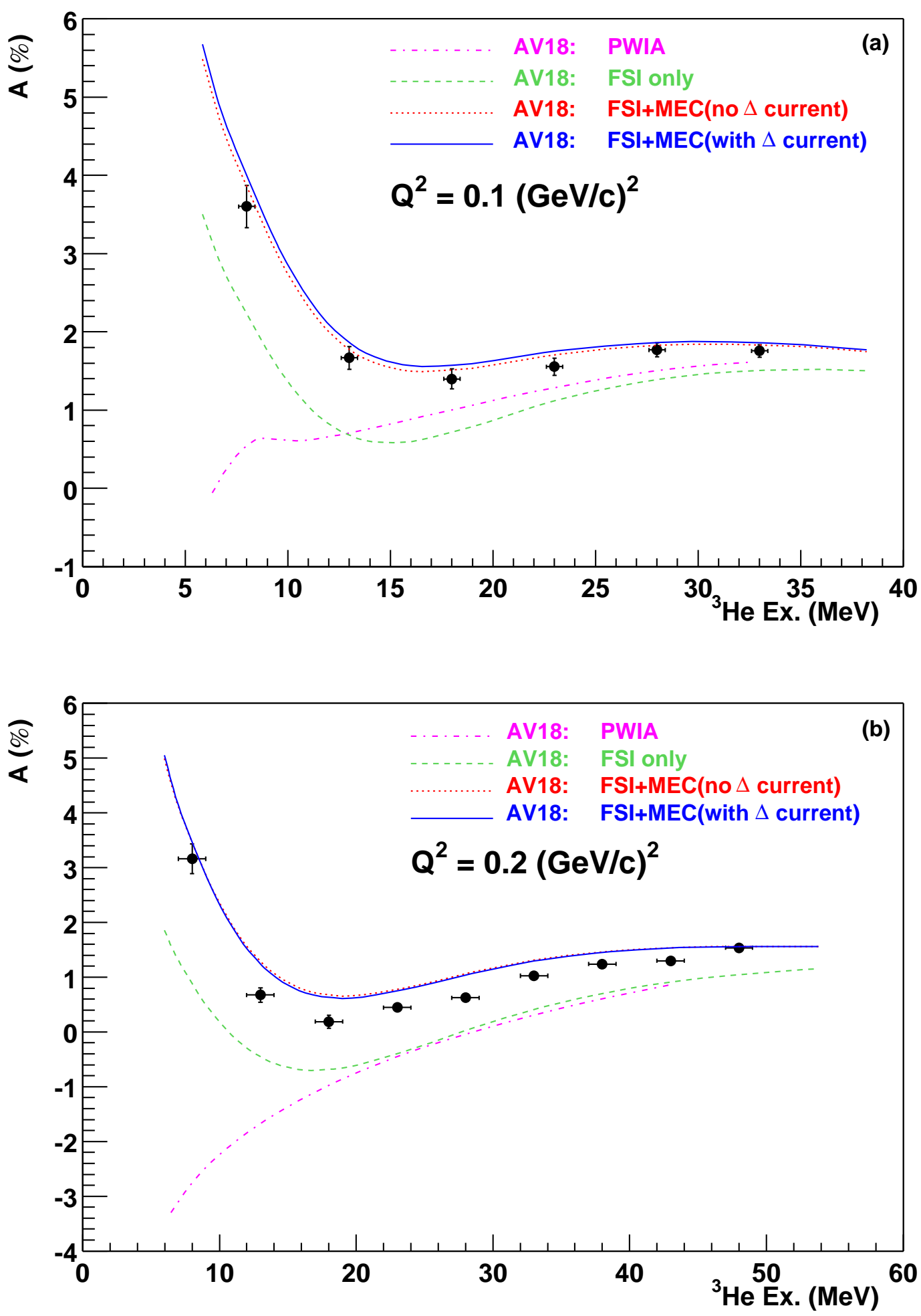

Figure 5-1: The physics asymmetry together with theoretical calculations for (a) $Q^{2}=0.1(\mathrm{GeV} / \mathrm{c})^{2}$ and $(\mathrm{b}) Q^{2}=0.2(\mathrm{GeV} / \mathrm{c})^{2}$. The theoretical calculations are all performed using AV 18 potential, but with different reaction mechanisms. The arrows point to the two-body and three-body breakup thresholds. 


\begin{tabular}{|ccccccc|}
\hline \hline $\begin{array}{c}E_{x} \\
(\mathrm{MeV})\end{array}$ & $\begin{array}{c}A \pm \delta^{\text {stat }} \\
(\%)\end{array}$ & $\begin{array}{c}\delta^{\text {pol }} \\
(\%)\end{array}$ & $\begin{array}{c}\delta^{\text {dil }} \\
(\%)\end{array}$ & $\begin{array}{c}\delta^{\text {ert }} \\
(\%)\end{array}$ & $\begin{array}{c}\delta^{r c} \\
(\%)\end{array}$ & $\begin{array}{c}\delta^{\text {acc }} \\
(\%)\end{array}$ \\
\hline 8.0 & $3.161 \pm 0.170$ & 0.121 & 0.070 & 0.121 & 0.018 & 0.014 \\
13.0 & $0.676 \pm 0.094$ & 0.044 & 0.022 & 0.064 & 0.034 & 0.011 \\
18.0 & $0.190 \pm 0.071$ & 0.022 & 0.010 & 0.036 & 0.075 & 0.035 \\
23.0 & $0.446 \pm 0.058$ & 0.020 & 0.008 & 0.021 & 0.012 & 0.021 \\
28.0 & $0.625 \pm 0.049$ & 0.019 & 0.006 & 0.012 & 0.006 & 0.021 \\
33.0 & $1.025 \pm 0.045$ & 0.024 & 0.007 & 0.007 & 0.012 & 0.017 \\
38.0 & $1.241 \pm 0.041$ & 0.026 & 0.007 & 0.005 & 0.015 & 0.013 \\
43.0 & $1.300 \pm 0.041$ & 0.026 & 0.006 & 0.005 & 0.018 & 0.011 \\
48.0 & $1.537 \pm 0.050$ & 0.028 & 0.005 & 0.005 & 0.020 & 0.022 \\
\hline
\end{tabular}

Table 5.2: The physics asymmetry at $Q^{2}=0.2(\mathrm{GeV} / \mathrm{c})^{2}$. Symbols are the same as in Table 5.1.

in the threshold region, the FSI and MEC effects are important. Indeed, the addition of FSI in the calculation improves the theoretical picture significantly. However, FSI alone is not enough to explain the discrepancy between the PWIA calculations and the data, and only full calculations which include both FSI and MEC effects can describe the data very well. This is not surprising because MEC effects are expected to be very strong in the threshold region. The MECs were chosen according to the Riska prescription [37], which relates nucleon-nucleon $(N N)$ forces and mesonexchange currents in a model-independent manner though the continuity equation. For AV18 NN force, the MECs are dominated by $\pi$ - and $\rho$ - exchange currents and more details can be found in [49]. To study the effects of model-dependent MECs, Faddeev calculations including additional exchange currents associated with $\Delta$-isobar degrees of freedom (the $\Delta$ current) were also carried out and compared with the Faddeev calculations without the $\Delta$ current. The difference is very small and suggests that model-dependent MECs have negligible effects.

It is interesting to study the MEC effects on individual response functions. Figure 5-2 compares Faddeev calculations with FSI effect only and full Faddeev calculations for all four response functions at $Q^{2}=0.1(\mathrm{GeV} / \mathrm{c})^{2}$, while Figure 5-3 shows 
the same comparison at $Q^{2}=0.2(\mathrm{GeV} / \mathrm{c})^{2}$. It can be seen that the MEC effects on $R_{T L^{\prime}}$ are the largest.

Theoretical uncertainties due to nucleon form factors, $G_{E}^{p}, G_{M}^{p}, G_{E}^{n}$ and $G_{M}^{n}$, were studied using PWIA $[6,83]$. The relative difference between the asymmetries calculated with the nucleon form factors from the Höhler parameterization and from experimental data [12-15,84-87] was found to be around 1\%, and thus theoretical uncertainties due to nucleon form factors are completely negligible.

To investigate the effects of different $N N$ potentials, we compare our data with full Faddeev calculations using the AV18 potential and the Bonn-B potential [27]. For the Bonn-B potential, standard $\pi$ - and $\rho$-meson exchange currents augmented by the strong form factors used in Bonn-B [49] are used in the full calculation. The Bonn-B potential and its closely related cousin, the CD-Bonn potential, are very different from other modern potentials in that they are fully non-local potentials while all other potentials apply local approximations one way or another. As a consequence, Bonn-B and CD-Bonn have a weaker tensor force than others. This is manifested in the predicted D-state probability, $P_{D}$, of deuteron. While CD-Bonn predicts $P_{D}=$ $4.85 \%$, the other potentials predict some larger value around $5.7 \%$. Thus by comparing theoretical calculations with two very different modern realistic potentials as AV18 and Bonn-B, we can get a very good sense of the dependence of the theoretical calculation on the choice of $N N$ potentials. The result is shown in Fig. 5-4. As can be seen, the difference between the theoretical calculations using these two potentials is very small, which suggests that for the kinematic region being considered, this observable is not sensitive to the choice of different $N N$ potentials and the corresponding exchange currents.

The good agreement between the full calculation and the data at $Q^{2}=0.1$ $(\mathrm{GeV} / \mathrm{c})^{2}$ suggests the validity of the current way of treating FSI and MEC in the full calculation. The small discrepancy at $Q^{2}=0.2(\mathrm{GeV} / \mathrm{c})^{2}$ may be due to the fact that some $Q^{2}$-dependent effects, such as the relativistic and $3 \mathrm{NF}$ effects, are not 


\section{MEC Effects: $Q^{2}=0.1(\mathrm{GeV} / \mathrm{c})^{2}$}
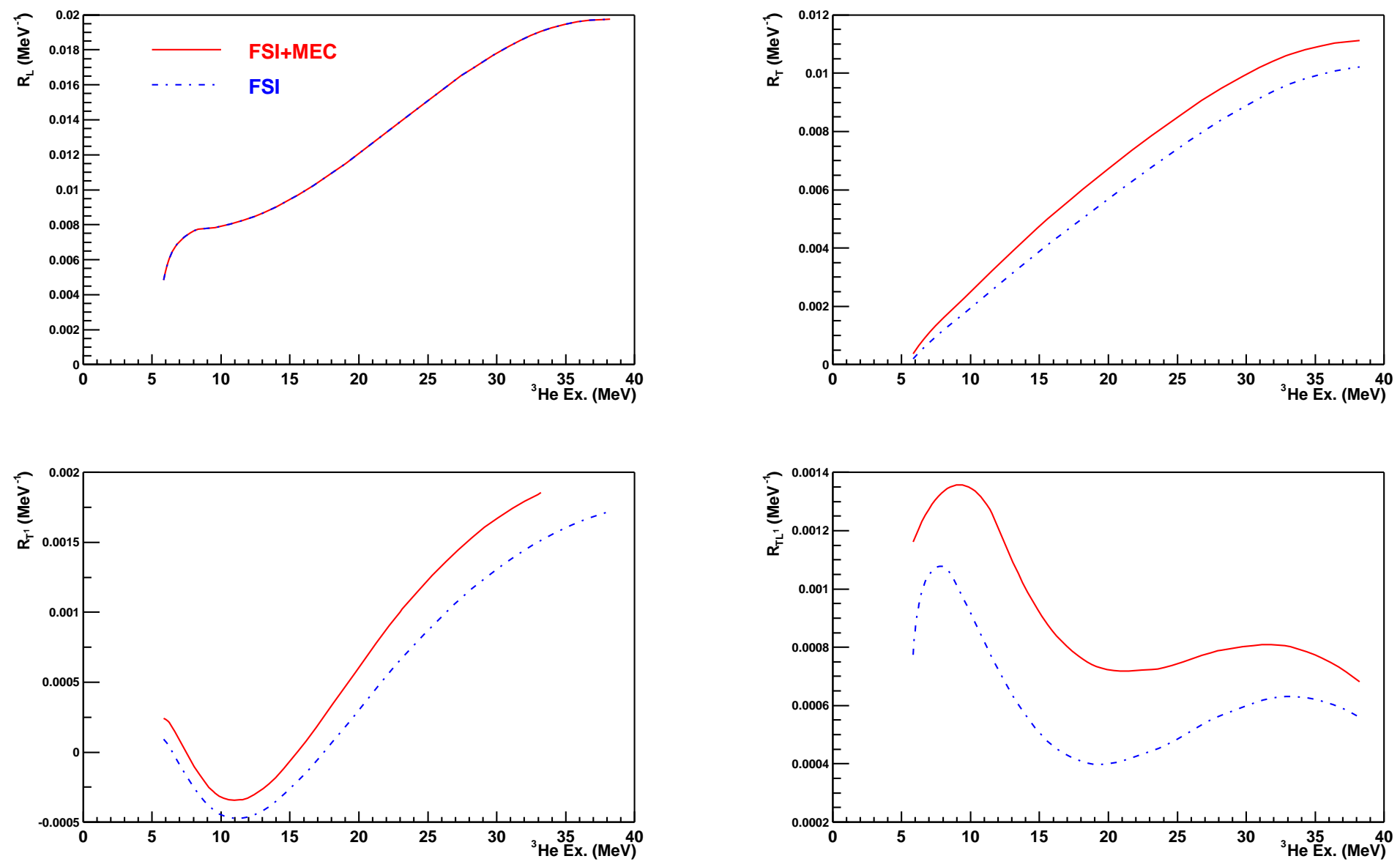

Figure 5-2: MEC effects on individual response functions at $Q^{2}=0.1(\mathrm{GeV} / \mathrm{c})^{2}$. 


\section{MEC Effects: $Q^{2}=0.2(\mathrm{GeV} / \mathrm{c})^{2}$}
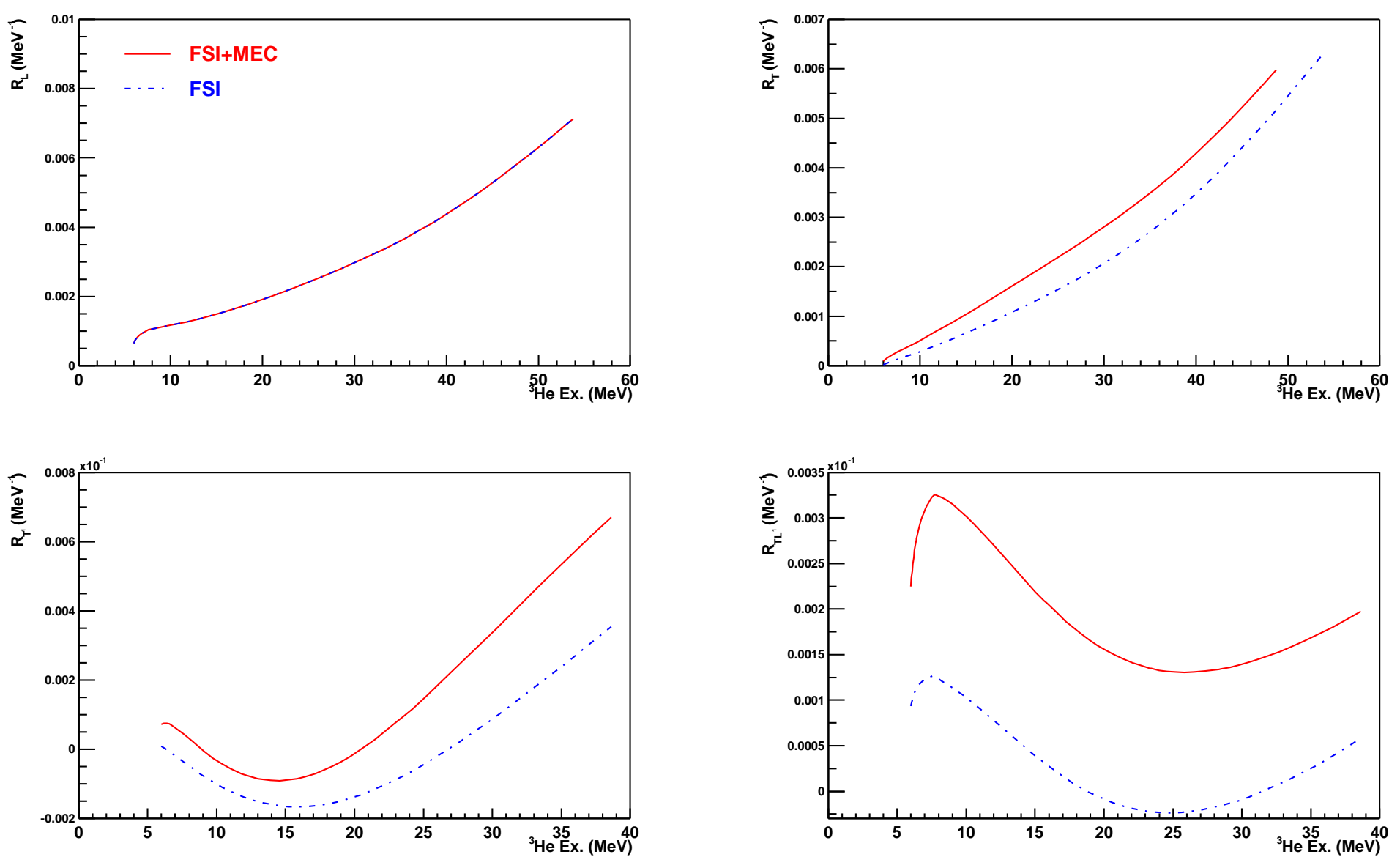

Figure 5-3: MEC effects on individual response functions at $Q^{2}=0.2(\mathrm{GeV} / \mathrm{c})^{2}$. 

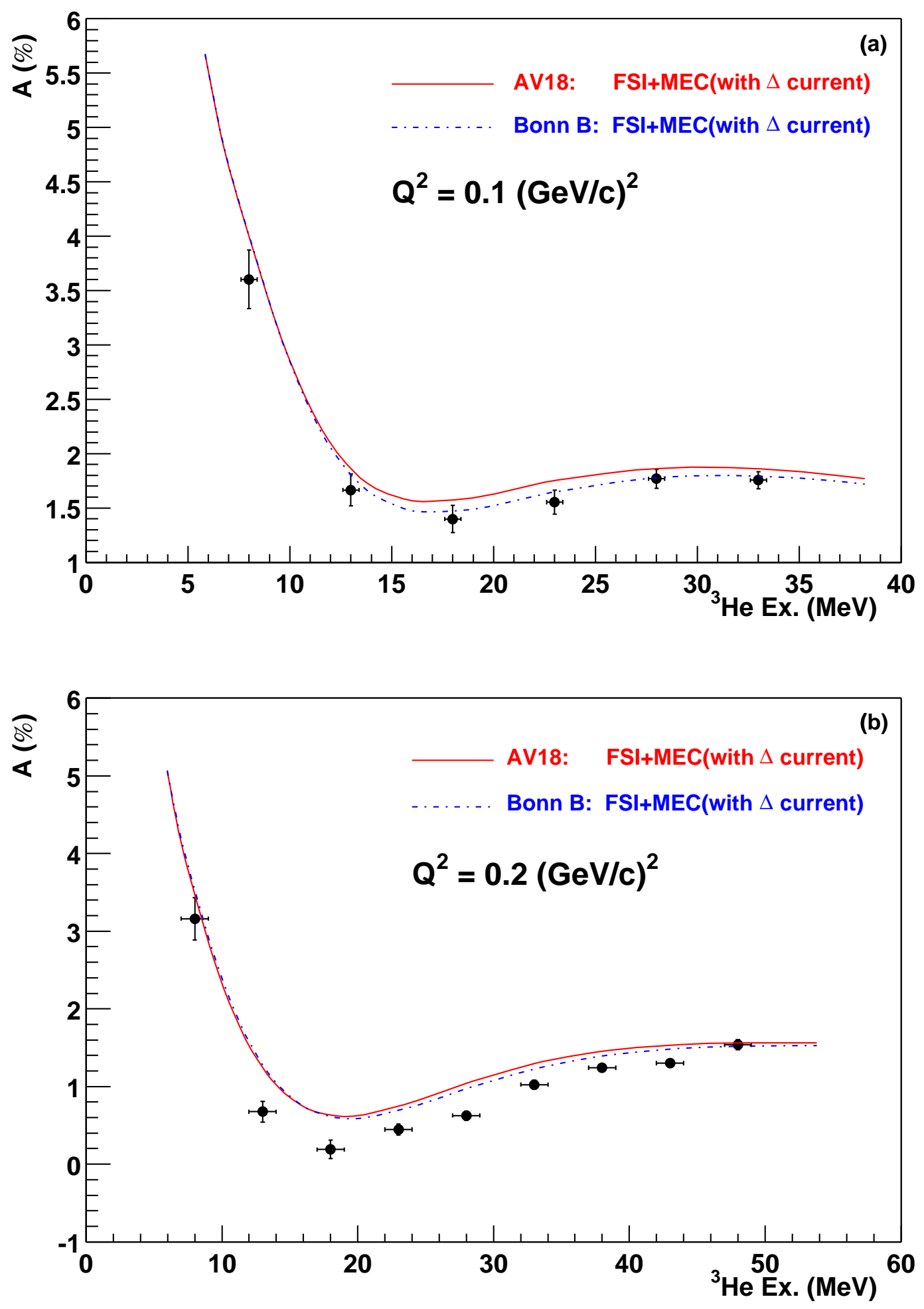

Figure 5-4: The physics asymmetry together with two full Faddeev calculations, one using AV18 as the $N N$ potential, the other using Bonn-B: (a) $Q^{2}=0.1(\mathrm{GeV} / \mathrm{c})^{2}$ and (b) $Q^{2}=0.2(\mathrm{GeV} / \mathrm{c})^{2}$. 
included in the current non-relativistic Faddeev calculation, which will be discussed in the following section.

\subsection{Theoretical Investigations}

Two of the most prominent ingredients missing from the current non-relativistic Faddeev calculations are relativistic corrections and three-nucleon (3NF) interactions. It has been shown that they are important in reproducing binding energies of light nuclei [35], and we would expect them to have large effects in scattering processes. Indeed, $3 \mathrm{NFs}$ are shown to be necessary to explain the spin observables $A_{y}, i T_{11}$ and $C_{y, y}$ in a recent proton-deuteron elastic scattering experiment [88]. Theoretically these two effects are related to each other and phenomenologically their contributions are comparable.

We will discuss each of these two effects, in particular their $Q^{2}$ dependence, and then an effective Chiral Lagrangian approach, in which these two effects occur naturally together in a systematic and controlled way through a power-counting scheme.

\subsubsection{Three-Nucleon Force Effects}

The conventional nuclear theory behind three-nucleon forces was explained in Chapter 1 and here we only study their effects on two-body and three-body breakup channels in this experiment. To investigate the $Q^{2}$-dependence of $3 \mathrm{NF}$ effects in two-body breakup ( $p d$ ) channel, one can study the $p d$ elastic scattering. It has been shown that in $N d$ elastic scattering the $3 \mathrm{NF}$ starts to take effect when the kinetic energy of the incident nucleon is around $60 \mathrm{MeV}$ [89], which corresponds to around $340 \mathrm{MeV}$ of $N d$ relative momentum, and becomes larger with higher energy. The $p d$ relative momentum in our experiment for two-body breakup channel was found to range from about $30 \mathrm{MeV}$ to $480 \mathrm{MeV}$ at $Q^{2}=0.1(\mathrm{GeV} / \mathrm{c})^{2}$, and from $0 \mathrm{MeV}$ up to $650 \mathrm{MeV}$ at $Q^{2}=$ $0.2(\mathrm{GeV} / \mathrm{c})^{2}$, thus one would expect the $3 \mathrm{NF}$ effects on two-body breakup channel 
to be larger at $Q^{2}=0.2(\mathrm{GeV} / \mathrm{c})^{2}$. Similarly, the $Q^{2}$-dependence of $3 \mathrm{NF}$ effects in three-body breakup (ppn) channel can be investigated by studying $p d$ breakup reaction. It has been found that in this channel the $3 \mathrm{NF}$ effects also increase with the energy of the incoming nucleon [90]. Thus we might expect the $3 \mathrm{NF}$ effects to be larger at higher $Q^{2}$ values in the threshold region of ${ }^{3} \mathrm{He}\left(\vec{e}, e^{\prime}\right)$.

Very recently Golak et al. [91] has been working to incorporate a $3 \mathrm{NF}$ into the framework of non-relativistic Faddeev calculations. The 3NF force was chosen to be Urbana IX, which is usually used with the AV18 nucleon-nucleon force. The Faddeev equation (1.74) in Chapter 1 now becomes [92]:

$$
U_{C}=\left(1+t G_{0}\right) C^{(1)}\left|\Psi_{3 \mathrm{He}}\right\rangle+\left(t G_{0} P+\left(1+t_{1} G_{0}\right) V_{4}^{(1)}(1+P) G_{0}\right) U_{C}
$$

where $V_{4}^{(1)}$ is the $3 \mathrm{~N}$ force. These Faddeev equations were then solved for our experimental conditions and the preliminary results for physics asymmetries at both $Q^{2}=$ 0.1 and $0.2(\mathrm{GeV} / \mathrm{c})^{2}$ are shown in Figure 5-5 together with the data and the same calculations without the inclusion of the $3 \mathrm{NF}$.

As can be seen, the preliminary results with $3 \mathrm{NF}$ are in much better agreement with the data at both kinematics. This suggests that $3 \mathrm{NF}$ does play an important role in describing the threshold region asymmetry. To better understand the effects of $3 \mathrm{NFs}$, we compare the response functions calculated with or without the inclusion of 3NFs in Figure 5-6 through Figure 5-9. We can see that the inclusion of 3NFs makes all response functions smaller, thus the $3 \mathrm{NF}$ effects turn out to cancel in forming the asymmetry, which suggests that the cross section might be a better observable to study the $3 \mathrm{NF}$ effects in the threshold region.

On the other hand, it is to be noted that theoretical calculations including 3NFs are still in the early stage and more work has to be done to thoroughly understand their effects. 

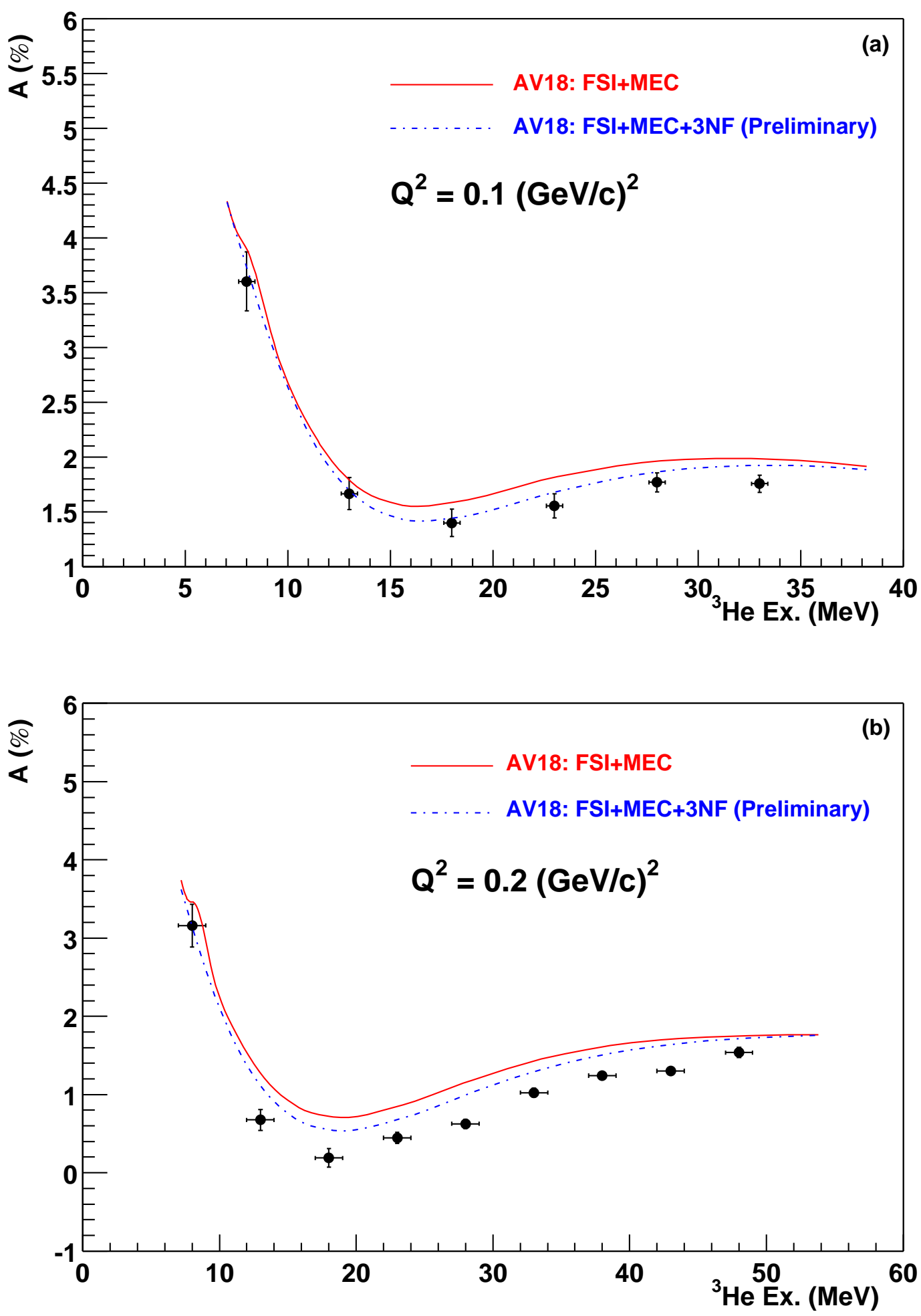

Figure 5-5: The physics asymmetry together with two full Faddeev calculations, one using AV18 as the $N N$ potential, the other using Bonn-B: (a) $Q^{2}=0.1(\mathrm{GeV} / \mathrm{c})^{2}$ and (b) $Q^{2}=0.2(\mathrm{GeV} / \mathrm{c})^{2}$. 

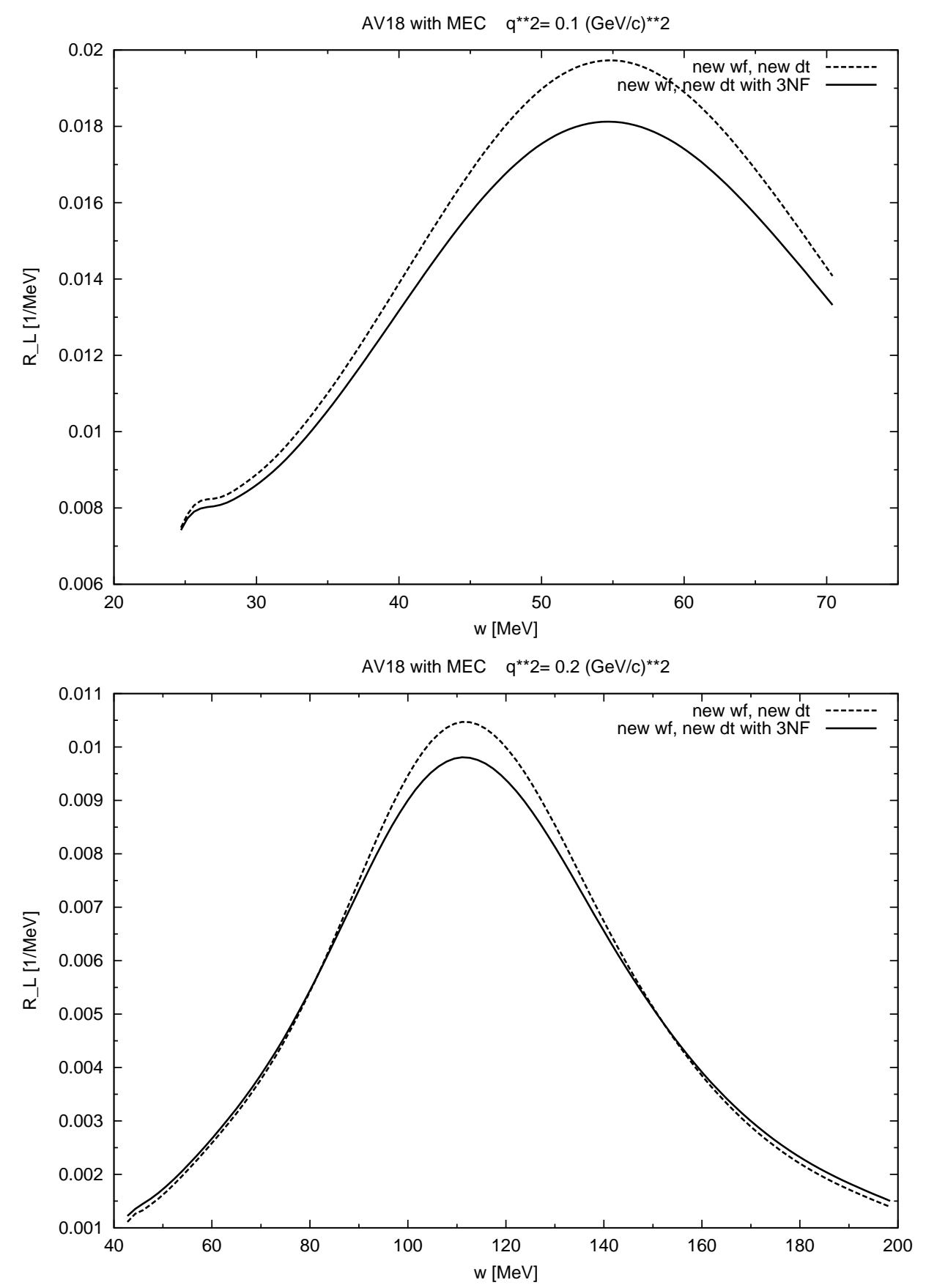

Figure 5-6: The effects of three-nucleon forces on $R_{L}$ at both $Q^{2}=0.1$ and 0.2 $(\mathrm{GeV} / \mathrm{c})^{2}$. 

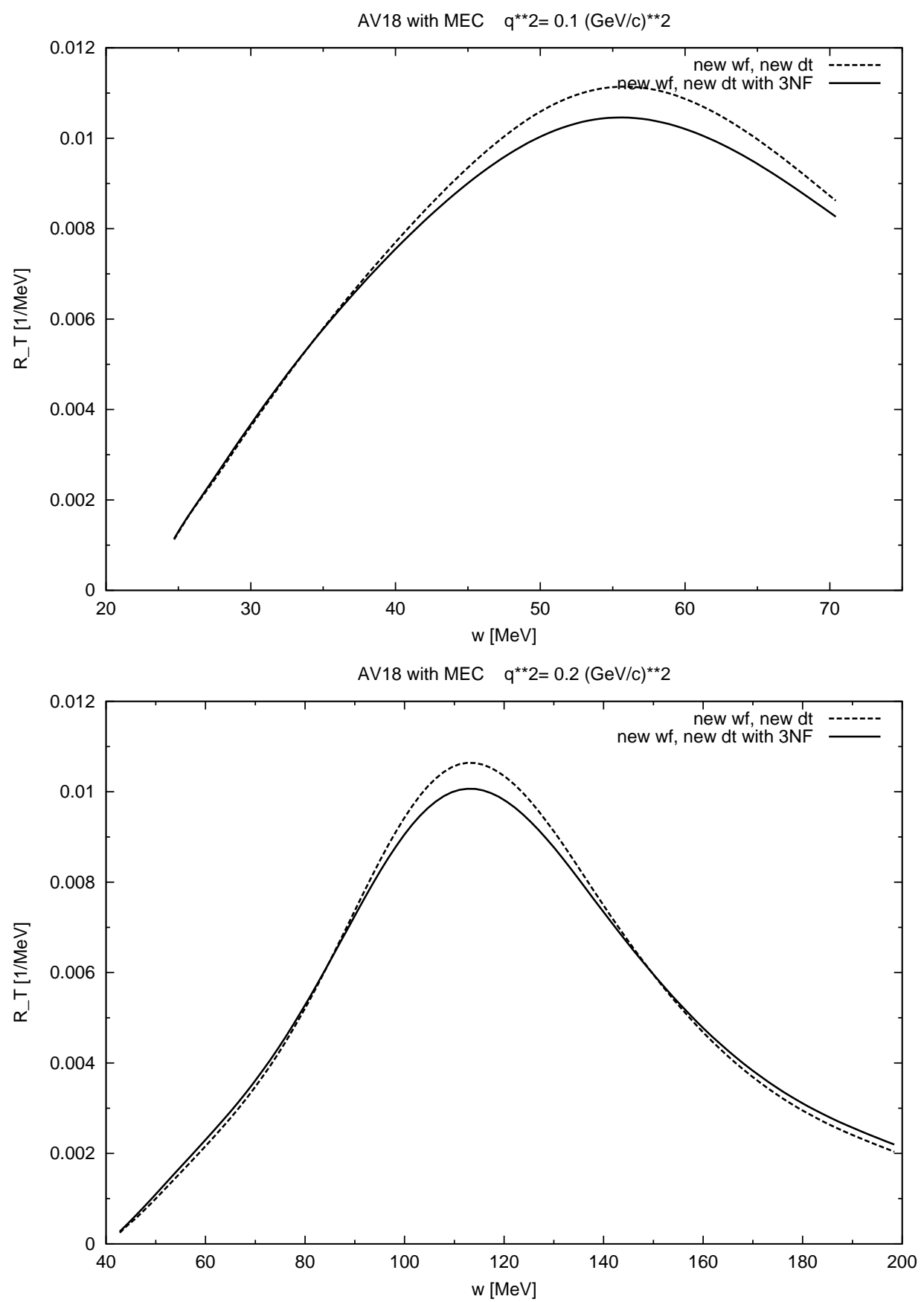

Figure 5-7: The effects of three-nucleon forces on $R_{T}$ at both $Q^{2}=0.1$ and 0.2 $(\mathrm{GeV} / \mathrm{c})^{2}$. 

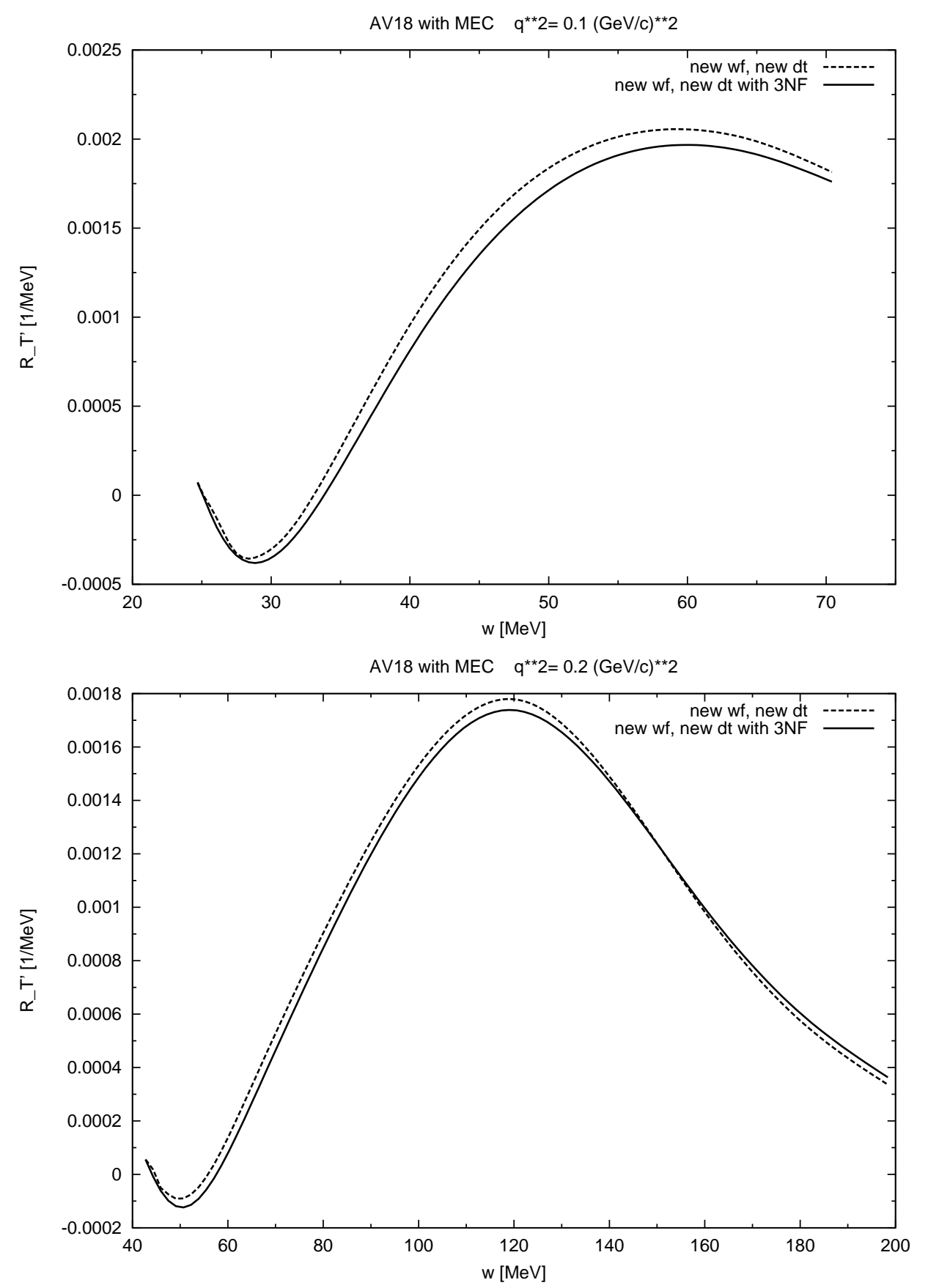

Figure 5-8: The effects of three-nucleon forces on $R_{T^{\prime}}$ at both $Q^{2}=0.1$ and 0.2 $(\mathrm{GeV} / \mathrm{c})^{2}$. 

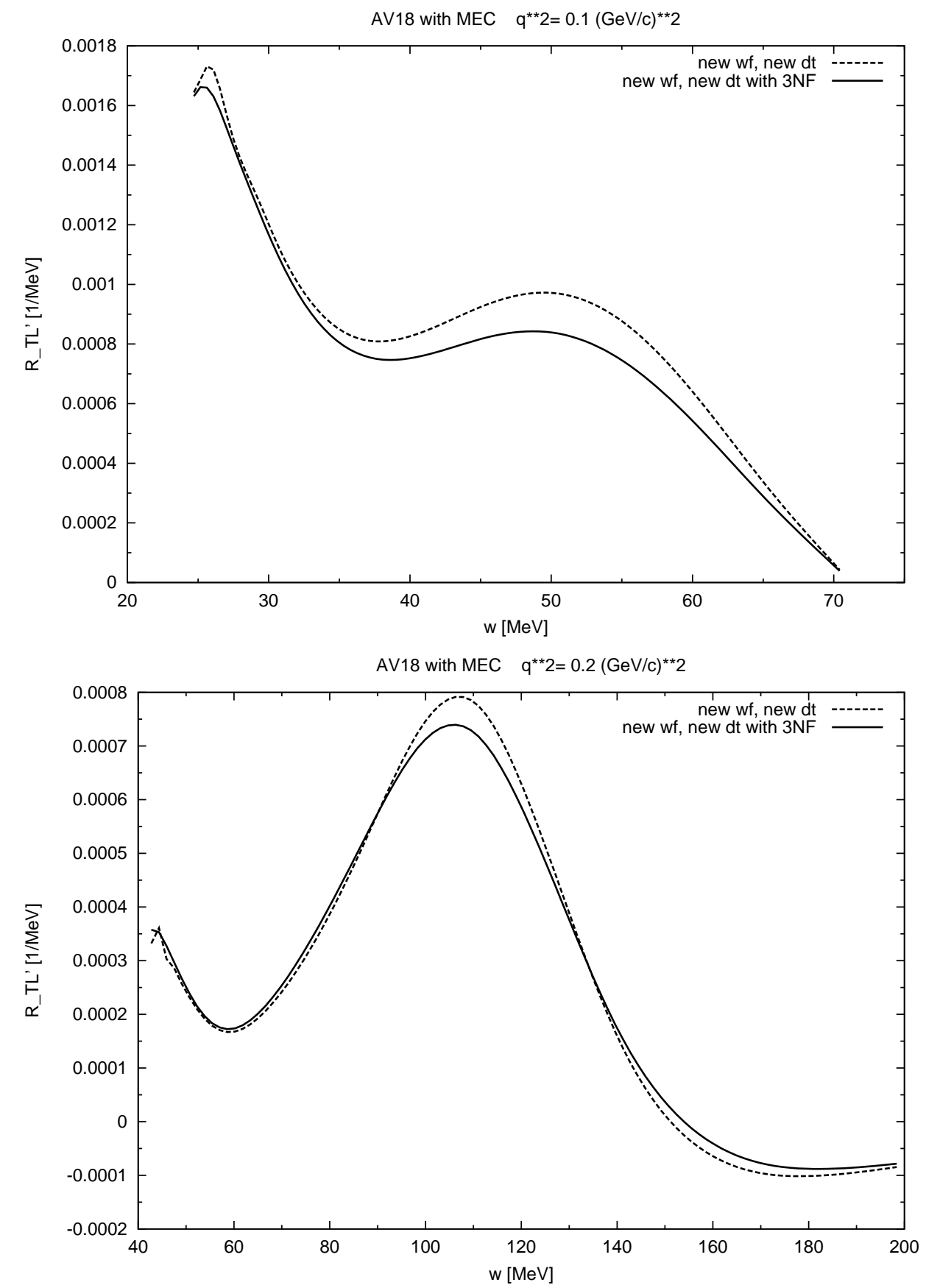

Figure 5-9: The effects of three-nucleon forces on $R_{T L^{\prime}}$ at both $Q^{2}=0.1$ and 0.2 $(\mathrm{GeV} / \mathrm{c})^{2}$. 


\subsubsection{Relativistic Effects}

It is natural to expect relativistic effects to be larger at higher $\mathrm{Q}^{2}$ values, and thus a theoretical calculation with relativistic correction is highly desirable to explain the discrepancy between theory and our data at $Q^{2}=0.2(\mathrm{GeV} / \mathrm{c})^{2}$. Unfortunately, it has proven to be notoriously difficult to perform Faddeev calculations in a relativistic framework.

One first step toward relativity is to use a relativistic current in the non-relativistic Faddeev equations [91]. However the problem with this approach is that the kinematics is not consistent with the current and the original way of calculating the t-matrix is no longer appropriate. Recently Kamada et al. [91] has been working on this problem and hopefully a scheme will emerge soon to calculate the t-matrix and build the proper kinematics in the Faddeev equations.

\subsubsection{Recent Development of Chiral Perturbation Theory}

It is important to note the recent development of chiral perturbation theory $(\chi \mathrm{PT})[93]$, an effective field theory of QCD based on the chiral symmetry present in QCD Lagrangian. $\chi \mathrm{PT}$ is essentially the low energy theory of QCD and can be calculated perturbatively in the sense of a certain power counting, and has been applied to a large variety of systems, such as pion physics. It can also be used to construct nuclear forces in a systematic and controlled manner [94].

Besides the aesthetic beauty, there is another very attractive point in this approach: both $3 \mathrm{NF}$ terms and relativistic corrections occur systematically in higher orders of perturbation series. Thus there is no need for manually including these effects as we have to do with the phenomenological models. The chiral $N N$ forces developed to next-to-leading order (NLO) have been applied to $3 \mathrm{~N}$ and $4 \mathrm{~N}$ systems by solving the Faddeev-Yakubovsky equations rigorously and surprisingly, the long standing $A_{y}$-puzzle in elastic $n d$ scattering seemed to be resolved [95]. More re- 
cently a next-to-next-leading (NNLO) calculation for three-body system has been performed [96] without the inclusion of $3 \mathrm{NFs}$, and the resulting description of $3 \mathrm{~N}$ observables is very poor, which shows that 3 NFs have to be taken into account at NNLO because of consistency in the power counting. Thus, unlike conventional models for $N N$ forces, which to a large extent describe the data well and 3NFs are only needed as a relatively small correction, $3 \mathrm{NF}$ s are an essential ingredient in $\chi \mathrm{PT}$.

It is thus necessary to perform NNLO calculations with the inclusion of 3NFs and probably even NNNLO calculations in the future, and it would be very interesting to observe how $3 \mathrm{NFs}$ and relativity occur naturally in these calculations and how they work together to describe the vast reservoir of recent experimental data in fewnucleon systems. In particular, and of more relevance to the present work, we would hope that in the future chiral $N N$ forces can be applied to electrodisintegration of ${ }^{3} \mathrm{He}$ in the threshold region.

\subsection{Summary and Conclusion}

In summary we have presented the first precision data on the spin-dependent asymmetry in the threshold region of ${ }^{3} \mathrm{He}\left(\vec{e}, e^{\prime}\right)$. The poor description of the data by PWIA calculations suggests the existence of strong FSI and MEC effects in this region. Indeed, the agreement between the data and non-relativistic Faddeev calculations which include both FSI and MEC effects is very good at $Q^{2}=0.1(\mathrm{GeV} / \mathrm{c})^{2}$. On the other hand, a small discrepancy at $Q^{2}=0.2(\mathrm{GeV} / \mathrm{c})^{2}$ is observed, which might be due to some $Q^{2}$ dependent effects such as relativity and three-nucleon forces, which are not included in the current framework of non-relativistic Faddeev calculations.

Some preliminary results show that their effects are indeed non-negligible in the threshold region and more theoretical work is currently underway towards a better understanding of both effects. A full Faddeev calculation within the framework of relativity which also include $3 \mathrm{NF}$ is highly desirable to understand our asymmetry 
results in the threshold region.

More recently, a completely new approach, called the chiral perturbation theory, has been developed and the resulting chiral $N N$ forces have been applied to threenucleon systems. Surprisingly, the long-standing $A_{y}$ puzzle seemed to be solved at next-to-leading order. Relativity and three-nucleon forces occur naturally in chiral perturbation theory through a power-counting scheme and it is hopeful that a future application of chiral $N N$ forces to electrodisintegration of ${ }^{3} \mathrm{He}$ might help us better understand our results. 


\section{Appendix A}

\section{Polarized Radiative Correction}

The $\mathrm{C}++$ simulation program, Polarized Radiative Correction (PRC), was built primarily to deal with the internal radiative correction for inclusive polarized electron scattering from polarized nuclear targets. Although only elastic and quasielastic scattering from ${ }^{3} \mathrm{He}$ and elastic scattering from ${ }^{4} \mathrm{He}$ and $\mathrm{N}_{2}$ are implemented, it is straightforward to add new targets and reaction channels due to the object-oriented feature of this program. In the following we will first discuss the class structure and then show how to run a sample main program.

\section{A.1 Class Structure and General Description}

The PRC program was built upon the platform of ROOT [97], which is the most popular data analysis package in high energy physics and recently has also been widely used in medium energy physics. As a result, most of its classes are derived from the TObject base class, as can be seen from the class inheritance tree shown in Figure A-1. PRCInclusive contains all important kinematics factors to describe a general inclusive electron scattering as either member variables or member functions. In addition, it contains all member functions useful to calculate the internal radiative correction for inclusive scattering of polarized electrons from polarized targets, 


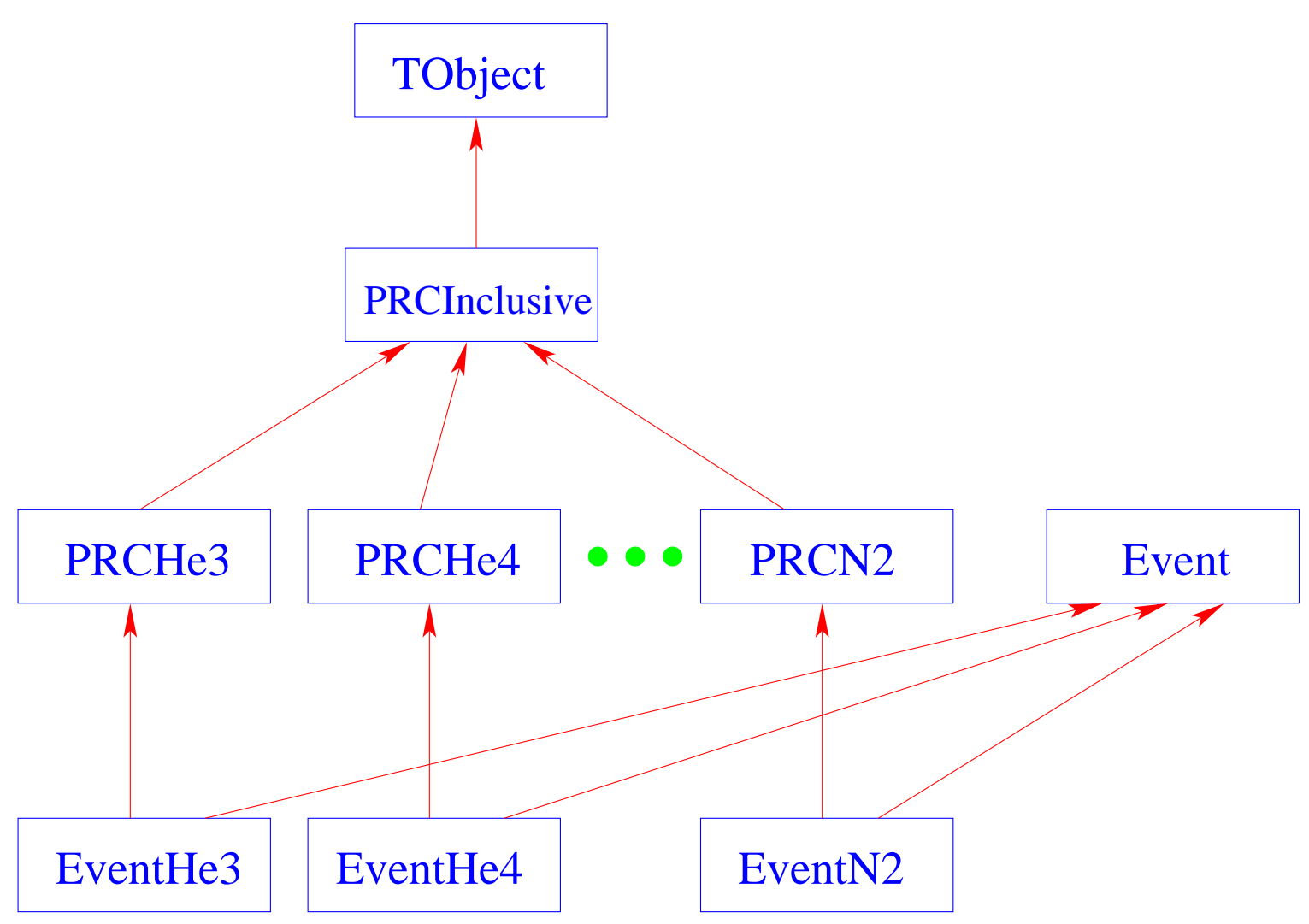

Figure A-1: The class inheritance structure for simulation program Polarized Radiative Correction (PRC).

for example, the $\theta_{i j}$ 's and their definite integrals discussed in Chapter 3. PRCHe3 describes inclusive scattering of polarized electrons from polarized ${ }^{3} \mathrm{He}$ in specific, and it contains member functions to calculate spin-dependent elastic cross section and quasielastic cross section of ${ }^{3} \mathrm{He}\left(\vec{e}, e^{\prime}\right)$, with elastic form factors and quasielastic response functions as inputs. It can be easily extended to include other reaction channels, such as deep inelastic scattering, with the additional knowledge of structure functions in these channels. Since PRCHe3 is inherited from PRCInclusive, it can use the latter's member functions to calculate radiative correction for ${ }^{3} \overrightarrow{\mathrm{He}}\left(\vec{e}, e^{\prime}\right)$. PRCHe4 and PRCN2 are similar classes for ${ }^{4} \mathrm{He}$ and $N_{2}$ targets, respectively. It should be noted that it is very straightforward to write similar classes for other targets, providing the appropriate form factors or structure functions as inputs. The 
class Event is a separate class describing a general simulation process, with member functions ranging from random number generators, to spectrometer transfer functions and magnet aperture cuts, to those calculating external radiative corrections. Some member functions, such as those related to the spectrometer and magnet apertures, are experiment-specific and must be modified for other experiments, while others, such as random number generators and functions for external radiative corrections, are portable. The classes EventHe3, EventHe4 and EventN2 are derived from the Event class and the PRC classes for each target, and their objects are used directly in main simulation programs, with access to all necessary member functions from their basis classes.

In the following we describe each of the above classes in detail.

\author{
TObject Base class in ROOT. It provides default behavior and protocol \\ for all objects in the ROOT system.
}

PRCInclusive Inherited from TObject. All kinematics factors and polarization variables necessary to describe a general polarized inclusive scattering are contained in this class as either member variables or member functions. The most important member functions in this class are those related to the internal radiative correction for an inclusive scattering of polarized electrons from polarized targets, for example, the $\theta_{i j}$ 's and their definite integrals discussed in Chapter 3. For the expressions of these complicated functions, please refer to [68]. 
PRCHe3 Inherited from PRCInclusive. The elastic ${ }^{3} \mathrm{He}\left(\vec{e}, e^{\prime}\right)$ cross section is calculated in a member function which calls a Fortran subroutine to determine the elastic form factor as a function of $Q^{2}$. The quasielastic ${ }^{3} \mathrm{He}\left(\vec{e}, e^{\prime}\right)$ cross section is calculated with a two-dimensional interpretation table for ${ }^{3} \mathrm{He}$ response functions from PWIA calculations or Full Faddeev calculations, obtained from an input file to be read in whenever a PRCHe3 object is instantiated. Elastic radiative tail and quasielastic cross section with internal radiative correction are also calculated by calling the member functions in PRCInclusive.

PRCHe4 Similar to PRCHe3, but with only member functions to calculate elastic cross sections.

PRCN2 Similar to PRCHe3, but with only member functions to calculate elastic cross sections.

Event This class contains member functions to generate random numbers from uniform distribution, Gauss distribution or Landau distribution. It also contains member functions to calculate the most probable energy loss, energy straggling, multiple scattering for incident or scattered electrons. Finally, it contains all spectrometer-related functions, such as spectrometer transfer functions and magnet aperture cuts, useful to transport the scattered electrons through the spectrometer and magnets to the focal plane.

EventHe3 Inherited from both PRCHe3 and Event.

EventHe4 Inherited from both $\mathrm{PRCHe} 4$ and Event.

EventN2 Inherited from both PRCN2 and Event. 


\section{A.2 Structure of a Main Simulation Program}

With the above classes at disposal, it is straightforward to write a main simulation program for a given target, desired reaction channels, and specified central kinematics.

First, an input file containing the kinematics, beam profile, target polarization angle, radiation options, event numbers for each reaction channel and other necessary information is read in.

Then, for each reaction channel an event loop will be run. Inside the loop, an event is generated as an object of the specific class, for example, EventHe3 if the target is ${ }^{3} \mathrm{He}$, with the initial beam energy, central kinematics and polarization information. A reaction point will be randomly generated inside the target volume and external radiative corrections are applied to the event for each material it passes through before reaching the reaction point. The scattering angles and final momentum (in the case of elastic scattering, the final momentum is calculated from scattering angles) are sampled in a range much larger than the experimental acceptance. After scattering, the event is transported to the entrance to the spectrometer and again external radiative corrections are applied for each material it passes through. The spectrometer transfer function will be used to transfer the event to each magnet aperture and test whether the event is inside the aperture. If the event falls outside of the aperture, it will be discarded and a new event will be generated. If the event goes through all magnet apertures and reaches the focal plane, some important weights, including cross section and asymmetry, will be calculated and written into an ntuple together with the kinematics information.

After the finish of all event loops, the ntuple will be saved in a ROOT file to be studied after the simulation. 


\section{Bibliography}

[1] W. Glöckle et al., Phys. Rep. 274, 107 (1996).

[2] H. Anklin et al., Nucl. Phys. 636, 189 (1998).

[3] J. Golak et al., Phys. Rev. C 52, 1216 (1995).

[4] J. Golak et al., Phys. Rev. C 62, 054005 (2000).

[5] B. Blankleider et al., Phys. Rev. C 29, 538 (1984).

[6] J.L. Friar et al., Phys. Rev. C 42, 2310 (1990).

[7] C. Ciofi degli Atti et al., Phys. Rev. C 46, R1591 (1992).

[8] R.-W. Schulze and P.U. Sauer, Phys. Rev. C 48, 38 (1993).

[9] C. Ciofi degli Atti et al., Phys. Rev. C 51, 1108 (1995).

[10] S. Ishikawa et al., Phys. Rev. C 57, 39 (1998).

[11] V.V. Kotlyar et al., Few-Body Syst. 28, 35 (2000) .

[12] W. Xu et al., Phys. Rev. Lett. 85, 2900 (2000).

[13] M. Meyerhoff et al., Phys. Lett. B 327, 201 (1994).

[14] J. Becker et al., Eur. Phys. J. A 6, 329 (1999).

[15] D. Rohe et al., Phys. Rev. Lett. 83, 4257 (1999). 
[16] P.L. Anthony et al., Phys. Rev. Lett. 71, 959 (1993); P.L. Anthony et al., Phys. Rev. D 54, 6620 (1996).

[17] K. Abe et al., Phys. Rev. Lett. 79, 26 (1997); K. Abe et al., Phys. Lett. B 404, 377 (1997).

[18] K. Ackerstaff et al., Phys. Lett. B 404, 383 (1997).

[19] Z.-E. Meziani et al., TJNAF Proposal No. E94-010; J.P. Chen et al., TJNAF Proposal No. E97-110.

[20] C. Ciofi degli Atti et al., Phys. Rev. C 48, R968 (1993).

[21] C. Ciofi degli Atti et al., Phys. Lett. B 404, 223 (1997).

[22] A. Amroun et al., Phys. Rev. Lett. 69, 253 (1992).

[23] G.A. Retzlaff et al., Phys. Rev. C 49, 1263 (1994).

[24] C.E. Jones et al., Phys. Rev. C 52, 1520 (1995).

[25] S. Weinberg, Nucl. Phys. B 363, 3 (1991).

[26] R.B. Wiringa et al., Phys. Rev. C 51, 38 (1995).

[27] R. Machleidt et al., Phys. Rev. C 53, R1483 (1996).

[28] P.J. Siemens and A.P. Vischer, Ann. Phys. (N.Y.) 238, 129 (1995); 238, 167 (1995).

[29] V.G.J. Stoks et al., Phys. Rev. C 49, 2950 (1994).

[30] J. Fujita and H. Miyazawa, Prog. Theor. Phys. 17, 360 (1957).

[31] B.S. Pudliner et al., Phys. Rev. Lett. 74, 4396 (1995).

[32] M.R. Robilotta et al., Nucl. Phys. A 451, 581 (1986). 
[33] S.A. Coon et al., Phys. Rev. C 48, 2559 (1993).

[34] D. Hüber et al., Few-Body Syst. 30, 95 (2001).

[35] J. Carlson and R. Schiavilla, Rev. Mod. Phys. 70, 743 (1998).

[36] J.D. Bjorken and S.D. Drell, Relativistic Quantum Mechanics, McGraw-Hill, New York (1964).

[37] D.O. Riska, Phys. Scr. 31, 107 (1985); Phys. Scr. 31, 471 (1985).

[38] J. Carlson et al., Phys. Rev. C 42, 830 (1990).

[39] M. Chemtob et al., Phys. Rev. C 10, 334 (1974).

[40] M. Gari and H. Hyuga, Nucl. Phys. A 264, 409 (1976).

[41] A.M. Green, Rep. Prog. Phys. 39, 1109 (1976).

[42] P.U. Sauer, Prog. Part. Nucl. Phys. 16, 35 (1986).

[43] J. Carlson and R. Schiavilla, Few-Body Syst. Suppl. 7, 349 (1994).

[44] F. Close, An Introduction to Quarks and Partons, Academic Press, New York (1979).

[45] T.W. Donnelly and A.S. Raskin, Ann. Phys. (N.Y.) 169, 247 (1986).

[46] H. Hajduk-Meier et al., Nucl. Phys. A 395, 332 (1983).

[47] S. Ishikawa et al., Nuovo Cimento A 107, 305 (1994).

[48] J. Golak et al., Phys. Rev. C 51, 1638 (1995).

[49] J. Golak et al., Phys. Rev. C 63, 034006 (2001).

[50] S. Ishikawa et al., Phys. Lett. B 339, 293 (1994). 
[51] W. Glöckle et al., The Quantum Mechanical Few-Body Problem, SpringerVerlag, Berlin (1983).

[52] S. Ishikawa et al., nucl-th/9909058.

[53] R. Alley et al., Nucl. Instr. Meth. A 365, 1 (1995).

[54] K. Unser, IEEE Transactions on Nuclear Science 16, 934 (1969).

[55] F.D. Colegrove et al., Phys. Rev. 132, 2561 (1963).

[56] W. Happer, Rev. Mod. Phys. 44, 169 (1972).

[57] S. Applet et al., Phys. Rev. A 58, 1412 (1998).

[58] M.V. Romalis, Ph.D. thesis, Princeton University (1997).

[59] I. Kominis, Ph.D. thesis, Princeton University (2001).

[60] A. Abragam, Principles of Nuclear Magnetism, Oxford University Press (1961).

[61] P. Ulmer, MCEEP User Manual 3.5 (2001).

[62] W.T. Scott, Rev. Mod. Phys. 35, 231 (1963).

[63] Particle Physics Booklet (2000).

[64] Y.S. Tsai et al., Phys. Rev. 122, 1898 (1961).

[65] J.A. Templon, Radiative-Tail Simulation for Mainz Expt. A1/1-93, (1998).

[66] J. Schwinger, Phys. Rev. 75, 898 (1949).

[67] L.W. Mo and Y.S. Tsai, Rev. Mod. Phys. 41, 205 (1969).

[68] I.V. Akushevich and N.M. Shumeiko, J. Phys. G 20, 513 (1994)

[69] D.Y. Bardin and N.M. Shumeiko, Nucl. Phys. B 127, 242 (1977). 
[70] A.A. Akuhundov et al., Yad. Fiz. 26, 1251 (1977).

[71] N.M. Shumeiko, Yad. Fiz. 29, 1571 (1979).

[72] T.V. Kukhto and N.M. Shumeiko, Nucl. Phys. B 219, 412 (1983).

[73] S. Penner, Nuclear Structure Physics, Proceedings of the 18th Scottish Univ. Summer School in Physics, 284 (1977).

[74] I.V. Akushevich et al., Eur. Phys. J. C 10, 681, (1999).

[75] X. Jiang, private communication.

[76] J. Lerose, private communication.

[77] A. Amroun et al., Nucl. Phys. A579, 596 (1994); C.R. Otterman et al., Nucl. Phys. A435, 688 (1985); P.C. Dunn et al., Phys. Rev. C 27, 71 (1983).

[78] N. Liyanaga, private communication.

[79] W. Xu, Ph.D. thesis, Massachusetts Institute of Technology (2002).

[80] J.S. Jensen, Ph.D. thesis, California Institute of Technology (2000).

[81] G. Höhler et al., Nucl. Phys. B114, 505 (1976).

[82] A. Kievsky et al., Phys. Rev. C 56, 64 (1997).

[83] J.-O. Hansen et al., Phys. Rev. Lett. 74, 654 (1995).

[84] T. Eden et al., Phys. Rev. C 50, R1749 (1994).

[85] M. Ostrick et al., Phys. Rev. Lett. 83, 276 (1999).

[86] C. Herberg et al., Eur. Phys. Jour. A 5, 131 (1999).

[87] I. Passchier et al., Phys. Rev. Lett. 82, 4988 (1999). 
[88] R.V. Cadman, Ph.D. thesis, University of Illinois at Urbana-Champaign (2001).

[89] H. Witała et al., Phys. Rev. Lett. 81, 1183 (1998).

[90] J. Kuroś-Żołnierczuk, private communication.

[91] J. Golak et al., private communication.

[92] J. Golak et al., nucl-th/0202014.

[93] B.R. Holstein, hep-ph/0001281.

[94] E. Epelbaum et al., Nucl. Phys. A 637, 107 (1998).

[95] E. Epelbaum et al., Phys. Rev. Lett. 86, 4787 (2001).

[96] E. Epelbaum et al., nucl-th/0201064.

[97] René Brun and Fons Rademakers, ROOT User's Guide 3.02b (2002). 